\title{
Experimental Study of Ion Heating and Acceleration During Magnetic Reconnection
}

\author{
Scott C. Hsu
}

\author{
A DISSERTATION \\ PRESENTED TO THE FACULTY \\ OF PRINCETON UNIVERSITY \\ IN CANDIDACY FOR THE DEGREE \\ OF DOCTOR OF PHILOSOPHY
}

RECOMMENDED FOR ACCEPTANCE

BY THE DEPARTMENT OF

ASTROPHYSICAL SCIENCES

JUNE 2000 
(C) Copyright by Scott C. Hsu, 2000. All rights reserved. 


\section{Abstract}

This dissertation reports an experimental study of ion heating and acceleration during magnetic reconnection, which is the annihilation and topological rearrangement of magnetic flux in a conductive plasma. Reconnection is invoked often to explain particle heating and acceleration in both laboratory and naturally occurring plasmas. However, a simultaneous account of reconnection and its associated energy conversion has been elusive due to the extreme inaccessibility of reconnection events, e.g. in the solar corona, the Earth's magnetosphere, or in fusion research plasmas. Experiments for this work were conducted on MRX (Magnetic Reconnection Experiment), which creates a plasma environment allowing the reconnection process to be isolated, reproduced, and diagnosed in detail.

Key findings of this work are the identification of local ion heating during magnetic reconnection and the determination that non-classical effects must provide the heating mechanism. Measured ion flows are sub-Alfvénic and can provide only slight viscous heating, and classical ion-electron interactions can be neglected due to the very long energy equipartition time. The plasma resistivity in the reconnection layer is seen to be enhanced over the classical value, and the ion heating is observed to scale with the enhancement factor, suggesting a relationship between the magnetic energy dissipation mechanism and the ion heating mechanism. The observation of non-classical ion heating during reconnection has significant implications for understanding the role played by non-classical dissipation mechanisms in generating "fast reconnection." The findings are relevant for many areas of space and laboratory plasma research, a prime example being the currently unsolved problem of solar coronal heating.

In the process of performing this work, local measurements of ion temperature and flows in a well-characterized reconnection layer were obtained for the first time in either laboratory or observational reconnection research. Furthermore, much progress was made in understanding the reconnection process itself. 

To my father

and

the memory of my mother 



\section{Acknowledgments}

I thank my advisers Drs. Masaaki Yamada and Hantao Ji for instructing me. Their dedication to high quality experimental plasma physics research has been a great inspiration. I am especially fortunate to have witnessed Masaaki's talent for identifying important research problems and for spearheading innovative plasma experiments. His suggestion to always "listen to the plasma" will not soon be forgotten. I am equally fortunate to have experienced Hantao's irrepressible scientific curiosity and his ability to be passionate yet objective about research. His untiring willingness to teach and encourage me on a daily basis have made my graduate education much more effective and highly enjoyable.

I thank Professor Russell Kulsrud and Dr. Richard Majeski for their critical reading of my thesis amidst very busy schedules. Their comments, criticisms, and suggestions have improved the rigor and clarity of the thesis. Of course, any shortcomings and errors left lurking are my own. I especially thank Russell for teaching me much about magnetic reconnection through his involvement with the MRX group. His presence at our meetings and discussions ensured that fundamental understanding was never pushed aside. His deep understanding of plasma physics helped guide our experiments on many occasions.

I thank the entire Program in Plasma Physics faculty for their efforts in teaching me plasma physics, and I thank Barbara Sarfaty for her cheer and concern for all of us graduate students and her unerring administrative prowess which allowed me to focus on my work. I thank Professor Steven Cowley of UCLA for a very fruitful second-year theory project on the equilibrium properties of very high-beta tokamaks, Drs. Philip Efthimion and Ira Lehrman for supervising my first-year experimental project on the construction of a free-electron laser, and Dr. William Tang for being a wonderful faculty adviser. I would also like to thank Professor Francis Chen of UCLA for introducing me to plasma physics as an undergraduate student through his engaging classes and for letting me work in his laboratory. 
I thank David Cylinder for his friendship and extraordinary instruction on all the hardware aspects of my work. His ability to create and to find solutions are talents not often surpassed. My experience working on MRX and living in New Jersey was made infinitely more enjoyable by his company, whether it be agonizing together over a probe that doesn't work and modifying it a half dozen times or going sailing with him and his wife Lorrie in his award winning, hand-built wooden boat. Because of Dave, my perception of life has been enriched.

Experimental research is almost always a group effort, and thanks go to all the MRX members with whom I have worked, especially Troy Carter, Dr. Haruhiko Himura, Dr. Dmitri Uzdensky (through his participation in MRX physics meetings), Dr. Fedor Trintchouk, Kyle Morrison, and Sorin Zaharia. I thank Dr. Gennady Fiksel of UW-Madison for generously loaning us his ingenious spectroscopy probe, which made the central measurements of this research possible, and Drs. Fred Levinton and Ron Bell for helpful discussions about plasma spectroscopy. Finally, I thank the dedicated PPL technical and engineering staff members who were always willing to bring me out of the dark ages on their subjects of expertise, most of all Jim Taylor, Ron Hatcher, Don Long, and Bob Marsala.

On a personal note, I will cherish the times spent in the company of Phil Snyder and Troy Carter, and more recently their respective spouses Mary and Becky. One could not otherwise comprehend the meaning of "the left side" nor appreciate the pleasure of a good old fashioned "spleen-busting." Also, the company of fellow classmates and friends through the years such as Ed Chao, Max Karasik, Tobin Munsat, Bryan Fong, Bob Heeter, Ernest Lo, and my officemate Jill Foley will be missed. I'd also like to express gratitude to all my extended family and good friends from California who have long supported my endeavors and sent patient wishes for my return. I thank my father and late mother who always encouraged me and gave me the freedom and opportunity to pursue what I loved.

Finally, I thank my wife Helen for her love and companionship and for the immense happiness she has brought me. This work is as much hers as my own.

I would like to acknowledge the financial support of the DOE Magnetic Fusion Science Fellowship Program (9/93-8/96) and the NASA Graduate Student Researchers Program (8/97-1/2000). 


\section{Contents}

Abstract $\quad$ iii

Acknowledgments vii

List of Figures $\quad$ xiii

List of Tables $\quad$ xvii

1 Introduction $\quad 1$

1.1 Motivation ...................... 2

1.1.1 Solar corona . . . . . . . . . . . . . . . . 2

1.1.2 Magnetosphere ................. . 3

1.1.3 Laboratory plasmas ................ 5

1.2 Reconnection and ion heating . . . . . . . . . . . . 6

1.2.1 Sweet-Parker model of reconnection . . . . . . . . . . . . . 6

1.2.2 Classical picture of the energy conversion process . . . . . . 9

1.2.3 Non-classical dissipation mechanisms . . . . . . . . . . . . . 10

1.3 Overview of previous work . . . . . . . . . . . . . . 11

1.3.1 Early experiments . . . . . . . . . . . . . . . 12

1.3 .2 UCLA experiments . . . . . . . . . . . . . . . . . 12

1.3.3 TS-3 experiments-Univ. of Tokyo . . . . . . . . . . . . . 13

1.3.4 Swarthmore Spheromak Experiment . . . . . . . . . . . 14

1.4 Dissertation objectives . . . . . . . . . . . . . . . 14

1.5 Dissertation findings and outline . . . . . . . . . . . . . 15

2 Experimental Apparatus $\quad 17$

2.1 Experimental setup . . . . . . . . . . . . . . 17 
2.1.1 Vacuum hardware . . . . . . . . . . . . . . . 17

2.1.2 Power system . . . . . . . . . . . . . . . . . . . . . . 19

2.1.3 Magnetic field coils . . . . . . . . . . . . . . 20

2.2 Plasma formation and achievable parameters . . . . . . . . . . 21

2.2.1 Plasma formation sequence. . . . . . . . . . . . . 21

2.2.2 Plasma parameters . . . . . . . . . . . . . . 26

2.3 Diagnostics . . . . . . . . . . . . . . . . . . . . . . . . . . . . 28

2.3.1 Magnetic probe arrays . . . . . . . . . . . . . . . . . 29

2.3.2 Electrostatic probes . . . . . . . . . . . . . . . 33

2.3.3 Ion Dynamics Spectroscopy Probe (IDSP) . . . . . . . . . . 40

2.3.4 Data acquisition system . . . . . . . . . . . 47

3 Characterizing the Reconnection Process 49

3.1 Magnetic field topology . . . . . . . . . . . . . . . 50

3.2 Thickness of the layer and current density profile . . . . . . . . . 53

3.3 Electron density and temperature profiles . . . . . . . . . . . . . 55

3.4 Reconnection rate . . . . . . . . . . . . . . . . . 57

3.5 Plasma resistivity . . . . . . . . . . . . . . . . . . . 59

3.6 Magnetic energy dissipation . . . . . . . . . . . . . . . 62

3.7 Highlight of MRX physics results . . . . . . . . . . . . . 64

3.7.1 Structure of the reconnection region . . . . . . . . . . . . 64

3.7.2 Verification of a generalized Sweet-Parker model . . . . . . . 67

3.7.3 Measurement of a Harris-like neutral sheet profile . . . . . . 70

3.8 Summary ............................. 73

4 Ion Heating and Acceleration During Reconnection 75

4.1 Identification of ion heating . . . . . . . . . . . . . . . . 77

4.1.1 Time evolution of ion temperature . . . . . . . . . . . 77

4.1.2 Radial profile of ion temperature rise . . . . . . . . . . . 86

4.2 Ion flow measurements . . . . . . . . . . . . . . . 88

4.2.1 Downstream flow . . . . . . . . . . . . . 88

4.2 .2 Toroidal flow . . . . . . . . . . . . . . . 91

4.3 Ion energy balance during reconnection . . . . . . . . . . . . . . 95

4.3.1 Reconnected field energy . . . . . . . . . . . . . 97 
4.3.2 Increase in ion thermal energy . . . . . . . . . . . . . . . 99

4.3.3 Compression . . . . . . . . . . . . . . . 100

4.3.4 Ion energy losses . . . . . . . . . . . . . . . . . 100

4.3.5 Classical viscous heating . . . . . . . . . . . . . . 103

4.3.6 Non-classical ion heating . . . . . . . . . . . . . . . . . . 104

4.4 Resistivity enhancement and ion heating . . . . . . . . . . . . . 105

4.5 Possible ion heating mechanisms . . . . . . . . . . . . . . . . 108

4.6 Summary . . . . . . . . . . . . . . . . . 110

5 Conclusions and Future Work 113

5.1 Conclusions .......................... 113

5.2 Future work . . . . . . . . . . . . . . . . . 117

A Independent Check of Mach Probe Measurements 119

B Plasma Perturbation due to the IDSP 123

C Doppler spectroscopy of helium ion spectral lines $\quad 127$

C.1 Principle of Doppler spectroscopy . . . . . . . . . . . . . . . . . . . 128

C.2 Other line broadening mechanisms . . . . . . . . . . . . . . . . . . 129

C.2.1 Instrumental broadening . . . . . . . . . . . . . . . . . . . . 129

C.2.2 Fine structure . . . . . . . . . . . . . . . . . . . 130

C.2.3 Pressure broadening . . . . . . . . . . . . . . . . 131

C.2.4 Plasma turbulence . . . . . . . . . . . . . . . . 131

C.2.5 Zeeman effect . . . . . . . . . . . . . . . . . 132

C.3 Curve-fitting of measured spectra . . . . . . . . . . . . . . . 132

$\begin{array}{ll}\text { Bibliography } & 135\end{array}$ 


\section{List of Figures}

1.1 Soft X-ray image of the solar corona . . . . . . . . . . . . 3

1.2 Illustration of the magnetosphere . . . . . . . . . . . . 4

1.3 Photograph of the aurora . . . . . . . . . . . . 5

1.4 Drawing of the Sweet-Parker reconnection layer $\ldots \ldots \ldots$

2.1 Photograph of MRX vacuum vessel . . . . . . . . . . . . 18

2.2 Schematic of MRX vacuum vessel . . . . . . . . . . . . . . . 19

2.3 Flux-core coil currents for pull reconnection . . . . . . . . . . 22

2.4 Drawing of plasma formation sequence and "pull" reconnection con-

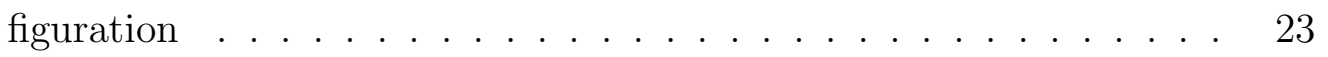

2.5 Photograph of plasma during pull reconnection $\ldots \ldots \ldots . . .24$

2.6 Illustration of null-helicity, co-helicity, and counter-helicity reconnec-

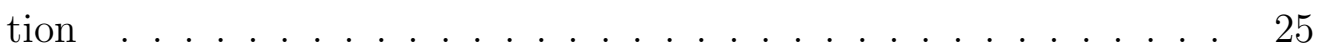

2.7 Setup of magnetic probe arrays, flux loops, and Rogowski coils . . . 30

2.8 Photograph of the high-resolution 1-D magnetic probe array . . . 31

2.9 2-D profile of reconnecting field measured by 90-channel probe array 33

2.10 Setup of triple Langmuir and Mach probes . . . . . . . . . . . 34

2.11 Schematic of triple Langmuir probe circuit . . . . . . . . . . 35

2.12 Photograph of the triple Langmuir probe . . . . . . . . . . 37

2.13 Photograph of the Mach probe . . . . . . . . . . . . . 39

2.14 Schematic of Mach probe circuit . . . . . . . . . . . . . 40

2.15 Schematic of the Ion Dynamics Spectroscopy Probe (IDSP) . . . . 42

2.16 Placement of the IDSP in MRX . . . . . . . . . . . 43

2.17 CCD camera image of He I $4713 \AA$ and He II $4686 \AA$ emission lines 44

2.18 Intensity versus CCD pixel plot of He II $4686 \AA$ line . . . . . . . . . 45

2.19 Ne I emission lines for wavelength per CCD pixel calibration . . . . 46 
3.1 Vector plots of poloidal magnetic field . . . . . . . . . . . 51

3.2 Contour plots of the poloidal magnetic flux function $\psi$. . . . . . . 52

3.3 High-resolution 1-D magnetic probe array measurements . . . . . . 54

3.4 Radial profiles of $B_{\mathrm{Z}}, j_{\theta}, n_{\mathrm{e}}$, and $T_{\mathrm{e}} \ldots \ldots \ldots \ldots$

3.5 Reconnection speed determined from motion of frozen-in flux and Mach probe . . . . . . . . . . . . . . . . 58

3.6 Resistivity enhancement as a function of collisionality . . . . . . . 60

3.7 Measured and classical resistivities versus density . . . . . . . . . 61

3.8 Comparison of terms in the simple Ohm's law for null-helicity reconnection . . . . . . . . . . . . . . . . . 62

3.9 Poloidal flux plots for null-helicity and co-helicity reconnection . . . 65

3.10 Verification of a generalized Sweet-Parker model . . . . . . . . . . . 69

3.11 Measurement of Harris-like neutral sheet profiles . . . . . . . . . . . 71

3.12 Scaling of current sheet thickness . . . . . . . . . . . . . . 72

4.1 Time evolution of $T_{\mathrm{i}}, T_{\mathrm{e}}$, and magnetic energy dissipation with and without reconnection (null-helicity) . . . . . . . . . . . . . 79

4.2 Time evolution of global $T_{\mathrm{i}}$ and local $T_{\mathrm{e}}$ (null-helicity) . . . . . . . 81

4.3 Time evolution of $T_{\mathrm{i}}, T_{\mathrm{e}}$, and magnetic energy dissipation with and without reconnection (co-helicity) . . . . . . . . . . . 82

4.4 Comparison of $T_{\mathrm{i}}$ time evolution for null-helicity and co-helicity . . 84

4.5 Comparison of time evolution of $E_{\theta}, j_{\theta}$, and $E_{\theta} j_{\theta}$ for null-helicity and co-helicity . . . . . . . . . . . . . . . 86

4.6 Spatial profile of ion heating and reconnecting field . . . . . . . . . 87

4.7 Downstream ion flow speed measured by Mach probe . . . . . . . . 89

4.8 Profile of density and electron temperature as function of $Z$ and time 90

4.9 Toroidal ion flow speed measured by IDSP (null-helicity) . . . . . . 92

4.10 Toroidal ion flow speed measured by IDSP (co-helicity) . . . . . . . 93

4.11 Radial profile of Langmuir probe floating potential as function of time (null-helicity) . . . . . . . . . . . . . . . . 95

4.12 Radial profile of Langmuir probe floating potential as function of time (co-helicity) . . . . . . . . . . . . . . . . 95

4.13 Reconnection electric field versus radius and time (null-helicity) . . 98 
4.14 Reconnection layer current density versus radius and time (nullhelicity $\ldots \ldots \ldots \ldots \ldots$. . . . . . . . . . . . . . . 98

4.15 Time evolution of density during null-helicity reconnection . . . . 99

4.16 Increase in ion thermal energy versus reconnected field energy . . . 100

4.17 Ion energy budget chart . . . . . . . . . . . . . . . . 106

4.18 Fractional ion heating and resistivity enhancement versus discharge voltage . . . . . . . . . . . . . . . . . . . . 107

4.19 Fractional ion heating versus resistivity enhancement . . . . . . 108

A.1 Measurement of toroidal ion flow using IDSP and Mach probe . . . 121

B.1 Effect of IDSP on magnetic field measurements . . . . . . . . . 124

B.2 Effect of IDSP on triple Langmuir probe measurements . . . . . . 125

B.3 Effect of IDSP on chord-averaged measurements of He II $4686 \AA$. . 126

C.1 Instrumental broadening profile . . . . . . . . . . . . . . 130

C.2 Doppler broadened He II $4686 \AA$ A spectral line . . . . . . . . . . . . 133 
xvi 


\section{List of Tables}

2.1 Relevant frequency and length scales in MRX plasmas . . . . . . . 27

2.2 Specifications of digitizers used for data acquisition . . . . . . . . . 47

4.1 Ion energy budget for null-helicity reconnection . . . . . . . . . . . 104 



\section{Chapter 1}

\section{Introduction}

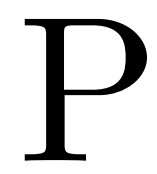

LASMA heating and acceleration mechanisms are often poorly understood in environments with strong magnetic activity, such as the solar corona, the Earth's magnetosphere, and laboratory plasmas such as reversed-field pinches, spheromaks, and field-reversed configurations. One of the most important mechanisms is commonly thought to be magnetic reconnection [VASYLIUNAS, 1975; BISKAMP, 1994], which is the topological rearrangement and annihilation of magnetic flux in a highly conductive plasma.

A detailed, simultaneous characterization of the energy conversion process and associated reconnection process has not emerged due to the extreme inaccessibility of reconnection events in both naturally occurring and laboratory plasmas. At present, solar coronal observations cannot resolve the spatial scales at which reconnection occurs, and magnetospheric satellites can only obtain data along one trajectory. In laboratory plasmas used for controlled fusion research, reconnection events are uncontrolled and unpredictable and occur on spatial scales also below the resolution of diagnostic capabilities. The dearth of clear measurements together with the ubiquity of energetic plasma phenomena attributable to reconnection provide motivation for the research presented in this dissertation, which seeks to identify and study ion heating and acceleration during magnetic reconnection in controlled laboratory experiments.

This introductory chapter is organized as follows. Section 1.1 provides motivation for this work by presenting three key examples of energetic plasmas found in environments with strong magnetic activity. Section 1.2 gives a physical description of reconnection and how it can heat and accelerate ions. Section 1.3 reviews 
Chapter 1. Introduction

previous experiments which have studied ion heating and acceleration during reconnection. Section 1.4 states the dissertation objectives, and Sec. 1.5 provides a summary and outline of the dissertation.

\subsection{Motivation}

Both in nature and in the laboratory, environments with strong magnetic activity are frequently populated by energetic plasmas which exhibit complex dynamics. How these plasmas are heated and accelerated is still a mystery in many instances. Below, three key examples are introduced.

\subsubsection{Solar corona}

The solar corona ${ }^{1}$ is the high temperature and low density "atmosphere" of the sun. It is highly fascinating, with bright active regions and associated dynamic magnetic structures. These are apparent in Fig. 1.1, which shows an image of the sun taken by the soft X-ray telescope onboard the Yohkoh satellite [Tsuneta et al., 1991].

One of the outstanding unsolved problems in solar physics research is how the coronal plasma is heated to more than $2,000,000^{\circ} \mathrm{K}$. A recent review [NARAIN and UlmschneIDER, 1996] offers a list of possible mechanisms, including shock dissipation of acoustic waves and slow mode MHD waves, Landau damping or turbulent heating of fast mode MHD waves and Alfvén waves, and dissipation of current sheets via magnetic reconnection. The latter mechanism is often studied in the context of solar flares, which are sudden "explosions" in the corona which release intense radiation from radio to gamma ray wavelengths. Both protons and electrons accelerated to relativistic energies have been detected near solar flares. It was realized early on [GiOvANELLI, 1939] in solar flare research that flares tend to occur near sunspot groups, which led to a theory involving particle acceleration near magnetic nulls as the source of flares [GiovanelLi, 1946]. As observational capabilities become better, the role of reconnection in powering solar flares and possibly heating the corona is becoming clearer. Recently, hard X-ray emission from loop tops and footpoints of compact flares were attributed to reconnection

\footnotetext{
${ }^{1}$ For detailed information on the solar corona and coronal phenomena, see e.g. [GoLuB and PASACHOFF, 1997] and references contained therein.
} 


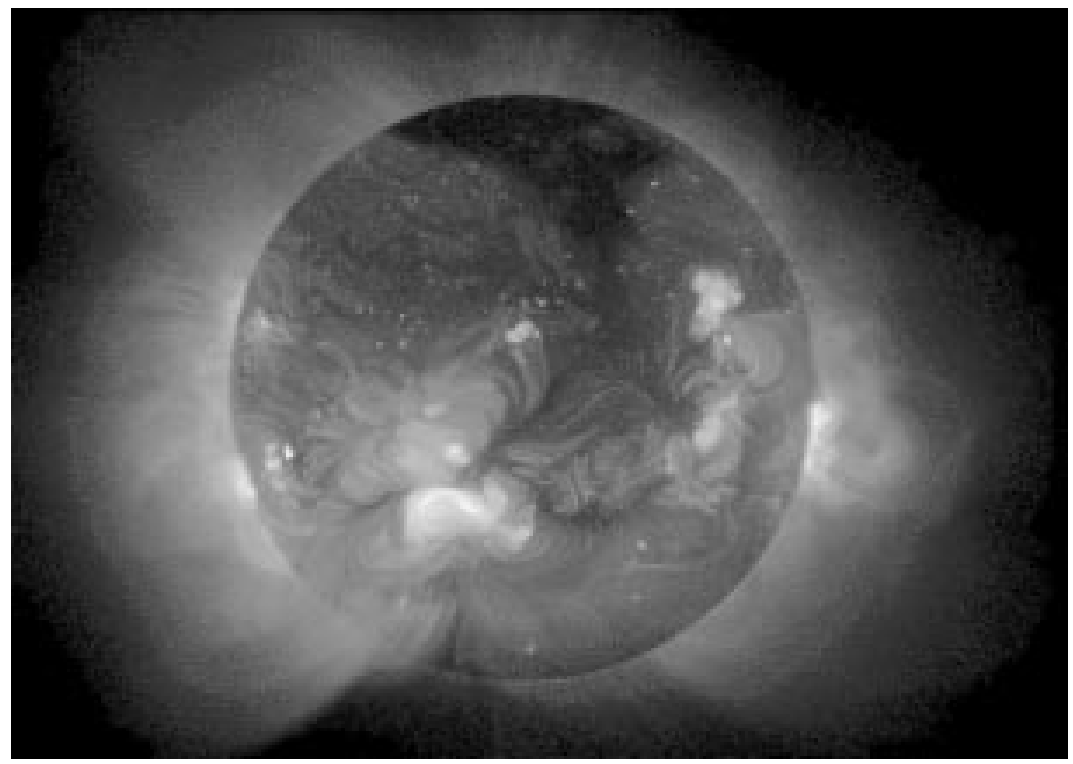

Figure 1.1: Soft X-ray image of the solar corona from the Yohkoh satellite. Visible are highly structured, bright active regions and coronal loops which hint at magnetic field structures and intense local heating.

[MASUDA et al., 1994], which has become favored by some as the most plausible coronal heating mechanism, e.g. [PRIEST et al., 1998].

The cause and effect between reconnection and particle acceleration and heating is extremely difficult to identify in the corona. The standard procedure is to interpret observational data based on reconnection theories which have not yet been proven to apply in the corona. Detailed physics, e.g. if and how reconnection accelerates ions and/or electrons in solar flares, must be studied with piecemeal (but very hard-won) data. Results from laboratory experiments, which can isolate reconnection and the energy conversion process and study them repetitively, can complement the observational data and answer many detailed questions about the possible role played by reconnection in solar flares and coronal heating.

\subsubsection{Magnetosphere}

The Earth's magnetosphere ${ }^{2}$ is a magnetic "cavity" created by the Earth's dipole field which sits in the path of the solar wind. An illustration of the wonderfully

\footnotetext{
${ }^{2}$ For detailed information on the magnetosphere and magnetospheric phenomena mentioned in this subsection, see e.g. [KIVELSON and RUSSELL, 1995] and references contain therein.
} 


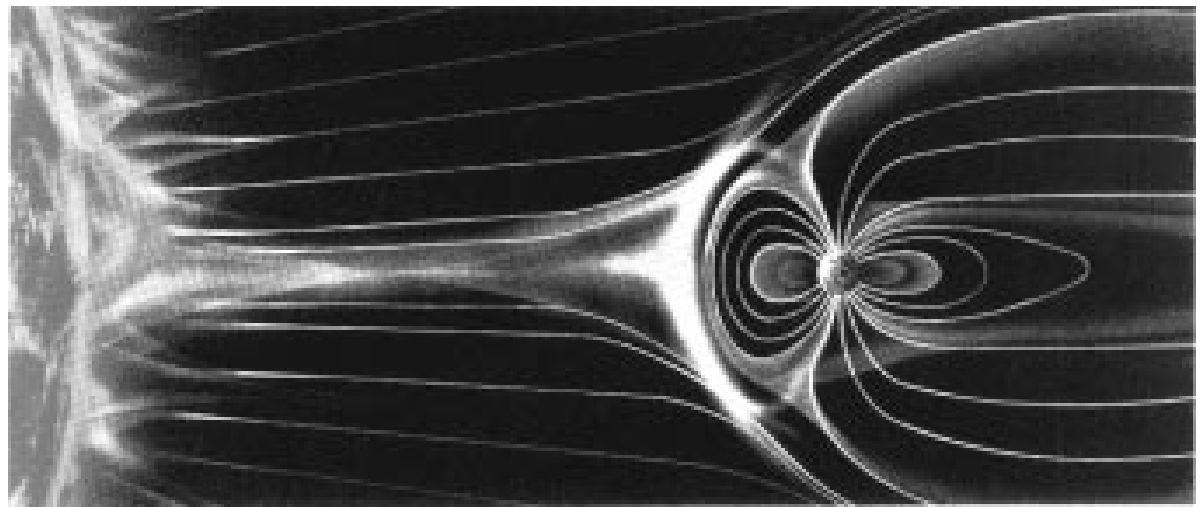

Figure 1.2: An artist's rendition of the Earth's magnetospheric cavity sitting in the path of the solar wind. Transient events in the solar corona are correlated strongly with geomagnetic storms and auroral phenomena in the magnetosphere.

complex magnetosphere (obviously not to scale) is shown in Fig. 1.2.

The magnetosphere is susceptible to large-scale magnetic disturbances known as auroral substorms and geomagnetic storms. This is becoming an urgent problem due to society's growing reliance on satellites, which can be damaged fatally by these events. The onset and triggering of such events is often studied in the context of reconnection [MCPherRon, 1979]. As first suggested by [DunGEy, 1961], reconnection at the dayside magnetopause allows energetic particles from the solar wind into the magnetosphere, which can lead to flux and energy buildup in the magnetotail. Ultimately, reconnection at the magnetotail can lead to the ejection of high speed plasmoids during the substorm. (One spectacular consequence of the auroral substorm is the intensification and breakup of the normally quiet aurora, as shown in Fig. 1.3.) The magnetosphere abounds with energetic particles, very often in correlation with magnetic activity levels. For example, the ion temperature is almost invariably seven times the electron temperature in the "plasma sheet" [BAUMJOHANn et al., 1989], which is earthward from the distant tail where reconnection occurs.

Determining how reconnection really contributes to magnetospheric dynamics and particle heating is a tough problem to study due to the immense physical size and turbulent nature of the magnetosphere. And due to the increasing human relevance of "space weather," the detailed laboratory study of one of the most fundamental magnetospheric processes, particle acceleration and heating during 


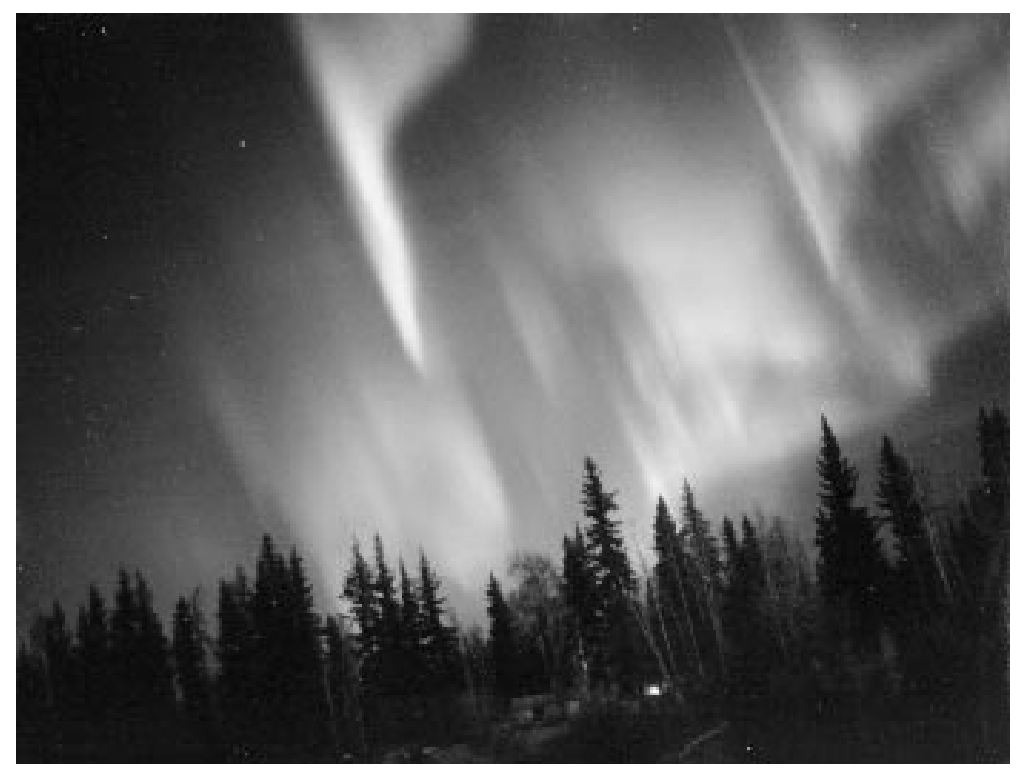

Figure 1.3: Photograph of the aurora, or "northern lights," which arises from collisions of energetic charged particles from the solar wind and the magnetosphere with atoms and molecules in the upper atmosphere.

reconnection, is especially timely.

\subsubsection{Laboratory plasmas}

Very early work on field-reversed configurations (FRC's) [TUszEWski, 1988] showed a correlation between reversed internal magnetic fields and neutron emission [KoLB et al., 1959; LiTTLE et al., 1961], implying intense ion acceleration. Since then, other plasma configurations utilized in controlled fusion research have had ion temperatures which are higher than electron temperatures even without auxiliary heating, including reversed-field pinches (RFP) [HOWELL and NAGAYAMA, 1985; FUJISAWA et al., 1991; SCIME et al., 1992b; SCIME et al., 1992a] and spheromaks [YAMADA et al., 1990a; MAYO et al., 1991].

Many hypotheses have been suggested to explain the "anomalous" ion heating mechanism(s), e.g. dissipation of energy in magnetic fluctuations at the ion cyclotron frequency [ScIme et al., 1992b; ScIme et al., 1992a] or viscous damping of ion kinetic energy gained by reconnection inherent in MHD dynamo activity [EJIRI and Miуamoтo, 1995]. The strong levels of magnetic fluctuations consistent with Taylor relaxation [TAYLOR, 1974; TAYLOR, 1986] in RFP and spheromak plasmas 
ensure that reconnection is likely to play an important role in the heating.

Since reconnection occurs unpredictably and on small scales in these fusion research plasmas, a systematic study between ion heating and reconnection in these plasmas is virtually impossible. Researchers can correlate the ion heating to dynamo activity and fluctuation levels but have very few ways of obtaining more details. The research in this dissertation can help clarify the role played by reconnection as an ion heating mechanism in laboratory plasmas. That is the very first step toward possibly controlling these confinement deteriorating fluctuations. Furthermore, understanding this process may have other untold potentials in future controlled fusion research.

\section{$1.2 \quad$ Reconnection and ion heating}

Magnetic reconnection is an active area of research with much yet to be understood. The interested reader is directed to several excellent reviews [VASYLIUNAS, 1975; BiskAMP, 1994; UzDENSKY, 1998; KulsRud, 1998], which give a sense of the scope and depth of the problem. In this sub-section, a simple description of reconnection is given, followed by discussions on how ions can be heated and accelerated by reconnection.

\subsubsection{Sweet-Parker model of reconnection}

The first quantitative model of reconnection was given in the seminal work of Sweet [SweEt, 1958] and Parker [PARKER, 1957], which was developed to explain the origin and evolution of solar flares. In this 2-D, steady-state, resistive MHD model, reconnection is envisioned to occur in a thin, narrow region called the reconnection layer, as shown in Fig. 1.4. Two topologically distinct plasmas with oppositely directed magnetic field lines are brought toward each other at speed $u$, creating a thin layer with an out-of-plane current sheet. The layer is characterized by a thickness $\delta$ and a length $L$. There is no magnetic field out of the page. Plasma is accelerated out of the layer at speed $V$. It is assumed that the plasma pressure is $p$ inside the layer and $p_{0}$ in both the upstream and downstream regions, and that the magnetic field is $B_{0}$ in the upstream region and zero along the center of the 


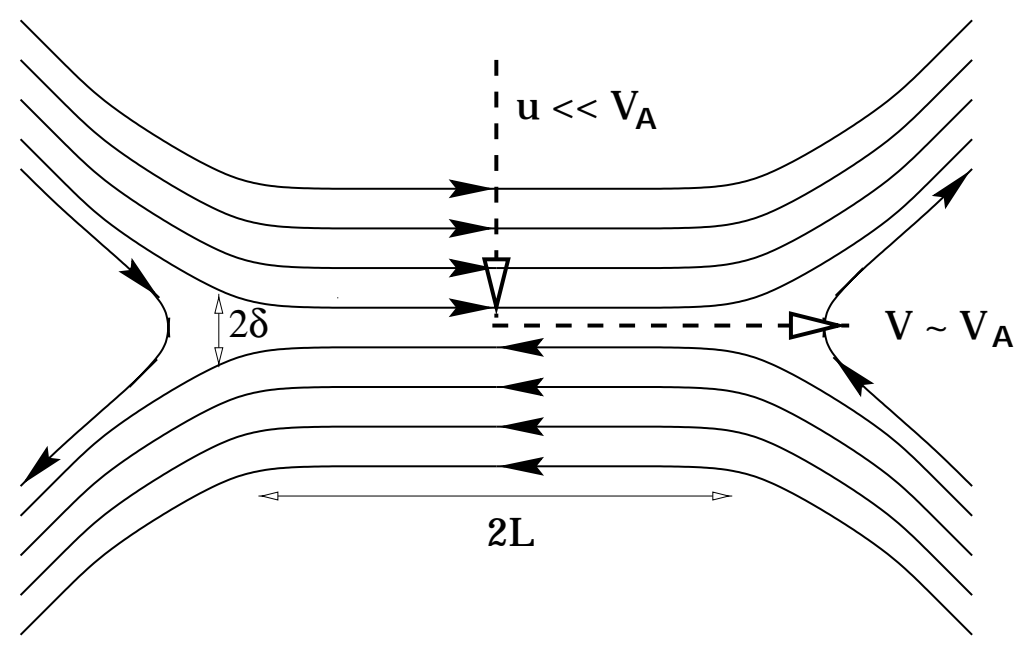

Figure 1.4: Sweet-Parker reconnection layer with thickness $\delta$ and length $L$. Lines with arrows represent magnetic field lines. The inflow speed $u$ of plasma and frozenin magnetic flux is much smaller than the Alfvén speed $V_{\mathrm{A}}$, while the outflow speed of plasma $V$ is on the order of $V_{\mathrm{A}}$.

reconnection layer.

An out-of-plane electric field exists, consistent with the simple Ohm's law

$$
\mathbf{E}+\mathbf{V} \times \mathbf{B}=\eta \mathbf{j}
$$

and is spatially uniform due to the 2-D and steady-state assumptions of the model. Here, $\eta$ is the classical Spitzer resistivity due to Coulomb collisions [SPITZER, 1962]. In the center of the reconnection layer, Eq. (1.1) simplifies to $E=\eta j \sim \eta B_{0} / \mu_{0} \delta$ since both $V$ and $B$ vanish there. In the upstream region, Eq. (1.1) becomes $E=u B_{0}$ since $j$ vanishes there. Because $E$ is spatially uniform, then the inflow speed is

$$
u=\frac{\eta}{\mu_{0} \delta}
$$

Pressure balance across and along the layer,

$$
p=p_{0}+\frac{B_{0}^{2}}{2 \mu_{0}}=p_{0}+\frac{1}{2} \rho V^{2}
$$

leads to an outflow speed of $V=B_{0} / \sqrt{\mu_{0} \rho} \equiv V_{\mathrm{A}}$, and mass conservation and the 
assumption of incompressibility give

$$
u L=V \delta
$$

Combining these results, a normalized reconnection rate is calculated to be

$$
\frac{u}{V_{\mathrm{A}}}=\left(\frac{\mu_{0} L V_{\mathrm{A}}}{\eta}\right)^{-1 / 2} \equiv S^{-1 / 2}
$$

where $S$, called the Lundquist number, is defined as the resistive diffusion time divided by the Alfvén transit time. In the solar corona and in the magnetosphere, $S$ is typically very large $\left(>10^{10}\right)$, meaning that the Sweet-Parker reconnection speed is infinitesimally slow compared to the Alfvén speed.

To summarize, the key features of the Sweet-Parker model of reconnection are the steady-state inflow of flux into a 2-D, narrow reconnection layer in which the field reverses direction. An out-of-plane current sheet develops, consistent with the sharp gradient of the field reversal. Resistive dissipation of the current is balanced exactly in steady-state by the out-of-plane electric field induced by the incoming magnetic flux. Equation (1.2) indicates that large resistivity and a narrow layer is needed to maximize the reconnection speed $u$. However, Eq. (1.4) shows that reducing $\delta$ limits $u$ at the same time due to the increased bottleneck for removing plasma mass from the layer. Thus, the Sweet-Parker reconnection rate is determined by the competing of effects of bringing in flux to be annihilated and in being able to remove the plasma quickly enough from the reconnection layer.

This simple physical model of reconnection captures many essential global features of a 2-D, steady-state reconnection process, such as a narrow layer and the existence of a current sheet. However, the slow reconnection speed predicted by the Sweet-Parker model is inconsistent with observations of solar flares, which evolve on near-Alfvénic time scales, and therefore the Sweet-Parker model has long been thought to be inapplicable to the problem of solar flares. In reality, MHD theory can break down in the reconnection layer if $B \rightarrow 0$ and ions are no longer magnetized, and "collisionless" effects which are neglected in Eq. (1.1) can become important. These exclusions, some of which are discussed in Sec. 1.2.3 lead to an incorrect 
treatment of the local plasma resistivity in the reconnection layer and hence a reconnection rate (and consequently a heating rate) which is too small. It should be mentioned that these details depend on the existence of an out-of-plane magnetic field, which incidentally plays no role in the 2-D model of Sweet and Parker.

\subsubsection{Classical picture of the energy conversion process}

The characteristics of energy conversion in the Sweet-Parker model are analyzed in this sub-section. The energy introduced into the reconnection layer per unit length out of the page (in Fig. 1.4) includes the kinetic energy of the inflowing plasma and the electromagnetic energy of the incoming field (Poynting flux). A simple estimate shows that

$$
\frac{\text { inflow kinetic }}{\text { electromagnetic in }}=\frac{\left(\rho u^{2} / 2\right) u L}{\left(E B_{0} / \mu_{0}\right) L}=\frac{u^{2}}{2 V_{\mathrm{A}}^{2}}=\frac{1}{2 S} \ll 1 \text {, }
$$

i.e. the inflow power is dominated by flux of magnetic energy ${ }^{3}$ since $S \gg 1$ in all cases of interest. This energy heats the current-carrying particles Ohmically, building up the pressure inside the layer to $p>p_{0}$ and creating a large $\nabla p$ along the layer. The pressure gradient accelerates the plasma out of the layer to $V=V_{\mathrm{A}}$, as was shown in Eq. (1.3). It can be shown that half of the incoming field energy is converted to kinetic energy of the Alfvénic outflow:

$$
\frac{\text { outflow kinetic }}{\text { electromagnetic in }}=\frac{\left(\rho V_{\mathrm{A}}^{2} / 2\right) V_{\mathrm{A}} \delta}{\left(u B_{0}^{2} / \mu_{0}\right) L}=\frac{V_{\mathrm{A}} \delta}{2 u L}=\frac{1}{2} \text {, }
$$

where Eq. (1.4) was used in the last equality. Therefore, in the Sweet-Parker model, half of the reconnected field energy goes to the thermal energy of the plasma inside the reconnection layer, and the other half goes to the kinetic energy of the Alfvénic outflow jet.

This single fluid MHD model does not distinguish between electrons and ions.

\footnotetext{
${ }^{3}$ In the denominator, $E=u B_{0}$ was used. In considering the electromagnetic energy, there is a factor of two difference between the estimate of the energy from the Poynting flux, $\left(E B_{0} / \mu_{0}\right) L=$ $u B_{0}^{2} L / \mu_{0}$, and the inflow of magnetic energy, $\left(B_{0}^{2} / 2 \mu_{0}\right) u L=u B_{0}^{2} L / 2 \mu_{0}$. The expression based on the Poynting flux must, of course, be correct. The extra factor of two comes from the work done by the incoming flux in pushing the flux in front of it out of the way. This can be derived rigorously by using the Maxwell stress tensor.
} 
Nevertheless, the model can be used to interpret a realistic steady-state reconnection process. Ohmic dissipation in the layer heats the electrons since they are likely to be the dominant current-carrying species due to their higher mobility. ${ }^{4}$ In this case, electrons are heated first but eventually equilibrate with the ions via Coulomb collisions, leading to a rise in ion temperature. Furthermore, an Alfvénic outflow jet, which can be accelerated immediately by the rise in electron pressure, can viscously damp on the background ions, heating the bulk ions downstream from the layer. Ion heating due to reconnection is often interpreted in this context both in space observations, e.g. [MASUDA et al., 1994], and laboratory experiments, e.g. [EJIRI and Міяамото, 1995]. However, as will be shown in this dissertation, the classical heating picture, while instructive, is unlikely to apply in any realistic reconnection scenario.

\subsubsection{Non-classical dissipation mechanisms}

It has long been realized that classical dissipation processes are not important in solar and space plasmas, which are highly collisionless. In this case, particles accelerated by the reconnection layer electric field can either (1) get scattered by electric fields set up by waves and instabilities (wave-particle interactions) or (2) have orbits which simply leave the current sheet ("inertial resistivity"). These effects can act essentially like a plasma resistivity, removing momentum from current-carrying particles, and heat the bulk plasma via processes such as Landau damping, viscosity, and gyro-resonance.

A review of all the waves and instabilities which have been proposed for collisionless reconnection is clearly beyond the scope of this introduction. However, two representative examples are (1) the lower-hybrid drift instability, which is a high frequency $\left(\omega \gg \Omega_{\mathrm{ci}}\right)$ drift wave driven by a cross-field current in the presence of a density gradient [HuBA et al., 1978] and (2) emission of Alfvén waves by thin current sheets in low $\beta$ plasmas [BELLAN, 1999] which can act as a resistivity [BELLAN, 1998] and Landau damp on ions. It is plausible that instabilities such

\footnotetext{
${ }^{4}$ However, the exact fraction of current carried by electrons versus ions in a reconnection layer is not always obvious. It depends strongly on whether the current is perpendicular or parallel with respect to the equilibrium field, i.e. it depends on the existence of an out-of-plane field. It may also depend on, for example, the exact field profile (if the current is strongly diamagnetic) and the potential profile (if $E \times B$ rotation is important).
} 
as these could account for the requisite resistivity and particle heating in realistic reconnection environments.

The concept of inertial resistivity in a collisionless current sheet was introduced by [SPEISER, 1970]. Physically, inertial resistivity arises when current-carrying particles exit the current sheet, limiting the current and dissipating some field energy in the process. No collisions of any kind are necessary to give rise to this effect. One example is due to particles drifting out of the current sheet due to a magnetic field perpendicular to the current sheet [SPEISER, 1965]. It has been suggested that such an effect in a reconnection current sheet with longitudinal magnetic field (parallel to current flow) may lead to a unified description for different regimes of particle acceleration in solar flares [Litvinenko, 1996]. Another example is "whistler mediated reconnection" [MANDT et al., 1994], in which a standing whistler mode in the reconnection layer transports electron momentum out of the layer. A hybrid (fluid electron and particle ions) simulation suggests that this type of collisionless reconnection can be Alfvénic even as the macroscopic scale length of the system becomes very large [SHAY et al., 1999]. Particles could attain a broad energy distribution via these inertial effects since they are accelerated in a random way inside the layer, and the plasma could appear to be heated virtually immediately upon entering the reconnection region.

The study of non-classical mechanisms both for the dissipation of magnetic field energy and for the heating of ions is very much an open research area at present.

\subsection{Overview of previous work}

Laboratory experiments have made important contributions toward understanding reconnection. In this section, past efforts to study ion heating and acceleration are reviewed briefly, and it is seen that steady progress was made toward being able to address the problem fully. However, clear cause and effect between reconnection and ion heating was not established, and truly local measurements of ion temperature in a fully diagnosed reconnection layer were not obtained, leaving the problem very much open. 


\subsubsection{Early experiments}

Early studies of ion heating in "magnetic neutral points" were performed in dense, pinch plasmas which lasted only a few micro-seconds. Current disruptions were common in these experiments, which are reviewed in [BAUM and BRATENAHL, 1977]. Strong claims were made, such as the identification [BRATENAHL and YeATES, 1970] of Petschek shocks [PETSCHEK, 1963] and turbulent resistivity [BAum and Bratenahl, 1973]. However, many of the results were interpreted with a lack of diagnostics. Also, the applicability of the results to the MHD theories with which they are compared is not straightforward (Lundquist number was very low, $S \sim 1$ ). In general, magnetic field measurements were not sufficient to determine the properties of a reconnection layer. Spectroscopic measurements, made in a "double inverse pinch" between two current-carrying rods, suggested sub-Alfvénic ion flows and slight ion heating near a neutral point [BAUM and BRATENAHL, 1974]. Anomalous resistivity and strong ion heating were reported in a similar neutral point discharge with four current-carrying conductors [OHYABU et al., 1974], but almost no information was given regarding the magnetic field. Particle acceleration was studied in "current sheet" $\theta$-pinch type devices [FRANK, 1989], and intensive plasma heating and generation of super-Alfvénic flows were reported. Based on the early work, it is clear that particles were accelerated and heated efficiently. However, due to the short time scales involved and lack of diagnostics, nothing definitive regarding the role of reconnection as a mechanism for ion heating and acceleration could be said in these early experiments.

\subsubsection{UCLA experiments}

Detailed magnetic field topology was first measured in the pioneering experiments at UCLA [STENZEL and GeKelman, 1981]. Experiments were performed in a linear device in the "electron MHD" regime, in which the characteristic scale length is $c / \omega_{\text {pe }}$ and ions are unmagnetized. In these experiments, detailed ion flow velocities were measured with electrostatic probes and noted to agree with the Sweet-Parker flow pattern. The fluid acceleration was a factor of three to five smaller than expected based on the measured $\mathbf{j} \times \mathbf{B}$ and $\nabla p$ forces, and scattering off wave turbulence was postulated to account for the difference. The nature of the turbulence was 
not identified, but it was noted that the frequency spectrum of fluctuations (maximum $\delta n / n \approx 20 \%$, maximum $\delta B / B \approx 100 \%$ ) was broadband, extending from MHD to lower hybrid $\left(\sqrt{f_{\mathrm{pe}} f_{\mathrm{pi}}} \sim 200 \mathrm{kHz}\right)$ and ion acoustic $\left(f_{\mathrm{pi}} \sim 300 \mathrm{MHz}\right)$ up to even the electron plasma frequency $(10 \mathrm{GHz})$ [GEKELMAN et al., 1982]. Electron heating and anomalous resistivity were also observed, and the resultant electron thermal energy was much larger than the ion flow energy [STENZEL et al., 1982]. However, ion temperature was not reported, and ion heating could not be addressed since there was no ion confinement. These experiments were comprehensive and excellently diagnosed, but application of the results to the full reconnection problem is not straightforward since ions play a critical role in reconnection dynamics.

\subsubsection{TS-3 experiments-Univ. of Tokyo}

Ion temperature rise in a fully diagnosed reconnection experiment was first measured on TS-3 at the University of Tokyo [ONo et al., 1996], also the first experiment to address MHD reconnection physics. In these colliding spheromak experiments, toroidal Alfvénic flows were believed to be accelerated by the tension force of reconnected field lines, and global ion heating to $200 \mathrm{eV}$ was attributed to viscous damping of the sheared Alfvénic flows [ONo et al., 1996; Ono et al., 1997]. However, it is unclear whether reconnection was indeed the dominant ion acceleration and heating mechanism. This is because the reconnection process was generated by the collision of two spheromaks traveling at a substantial fraction of the Alfvén speed. Compressional heating or conversion of the spheromak translational energy via other mechanisms were not ruled out. Some uncertainty exists due to the unfolding of the chord-averaged polychromator measurements. In the presence of a large flow velocity, accurate (and local) values of both ion temperature and flow velocity can only be obtained through a proper Abel-inversion technique [BELL, 1997], but this is very difficult to perform. Additionally, the spectroscopy was based on neutral $\left(\mathrm{H}_{\beta}\right)$ and impurity ion (C II) emission, respectively, requiring assumptions regarding charge-exchange and ion-impurity energy equilibration in order to infer information about the majority ions. Despite these questions, it is still likely that the majority ions were heated globally due to viscous damping of energetic flows driven by the reconnection process. 


\subsubsection{Swarthmore Spheromak Experiment}

In another colliding spheromak experiment, SSX (Swarthmore Spheromak Experiment), ${ }^{5}$ Alfvénic ion jets downstream from the reconnection layer were reported based on measurements of ion flux by a retarding grid energy analyzer (RGEA) situated at the vacuum wall [KorNACK et al., 1998]. When the RGEA was oriented to collect ions from the upstream region, only thermal ions were detected. The RGEA is an electrostatic probe with a negatively biased grid to repel electrons, then a variable positively biased grid to select ions, and a negatively biased collector plate to collect ion current. The resulting $I-V$ characteristic can yield ion temperature and directed flow information. The SSX results are consistent with the flow patterns of the classical reconnection picture. This experiment must also contend with the ambiguity introduced by the high translational energy of the initial colliding spheromaks, which interact at a gap in the flux conservers at the midplane. The gap in the flux conservers also may play a role in limiting the removal of reconnected flux. This could change the data interpretation. Also, it might be mentioned that there is inherent ambiguity in fitting a single RGEA $I-V$ characteristic to an ion distribution with both drift and thermal components, and more data would solidify the results reported.

\subsection{Dissertation objectives}

The primary objectives of this dissertation were to study ion heating and acceleration during magnetic reconnection in a controlled and well-diagnosed laboratory experiment. The lack of a detailed experimental characterization of this process represented a gaping hole in reconnection research. The experimental goals were to determine (1) if ion heating during reconnection could be observed, (2) whether the observed ion heating could be causally linked to the reconnection process itself, (3) whether energetic ion flows existed and could serve as an important ion heating mechanism, and (4) the nature of the ion heating mechanism(s). Two significant improvements on previous work are the ability to obtain local measurements of ion temperature and the improved way in which reconnection can be studied. The

\footnotetext{
${ }^{5}$ SSX came online after MRX, but their results on ion acceleration came before this work.
} 
latter is due to the experimental capability of MRX (Magnetic Reconnection Experiment), on which experiments for this dissertation were performed. In MRX, 2-D reconnection layers are formed quasistatically, allowing for truly isolated and versatile studies of the reconnection process. A further distinguishing feature of this research is the thorough characterization of magnetic field topology with more than 135 internal magnetic probes.

The entirety of this research consisted of the following: (1) helping bring MRX up to full research capabilities, (2) performing experiments to characterize the reconnection process in detail, (3) developing and implementing low-cost diagnostics for measuring local ion temperature and flow velocity, and (4) performing experiments to identify and investigate ion heating and acceleration during reconnection.

\subsection{Dissertation findings and outline}

The main findings of this research are the identification of ion heating as an unambiguous consequence of magnetic reconnection and the occurrence of the heating predominantly through non-classical dissipation mechanisms. Ion acceleration is measured and shown to be energetically small, and the associated viscous heating by the flows is also small. There is some indication that ion heating scales with the enhancement of resistivity over the Spitzer value, possibly indicative of waveparticle interactions providing the non-classical dissipation and heating, but other collisionless effects such as inertial resistivity cannot be ruled out. Following is an outline of the dissertation.

Chapter 1. This introductory chapter motivates the research by presenting examples of where ion heating via magnetic reconnection could be important in nature and in the laboratory. Then a physical description of reconnection and how it could heat and accelerate ions is given, followed by an overview of previous work done. Finally, the dissertation objectives and findings are summarized.

Chapter 2. This chapter describes the experimental apparatus of the Magnetic Reconnection Experiment, including details about diagnostics. 
Chapter 3. This chapter describes the reconnection process realized in MRX, showing in detail the magnetic field and electron temperature and density measurements, and how relevant reconnection physics quantities are derived from the data. Key physics results from MRX are summarized.

Chapter 4. This chapter describes the ion heating and acceleration experimental measurements. It is shown that ions are heated directly due to the reconnection process, and that ion flow speeds are sub-Alfvénic, consistent with the observed high downstream pressure. An energy ion energy balance is presented, showing that the ion heating must have occurred predominantly via non-classical mechanisms. There are indications that ion heating scales with resistivity enhancement over the classical value.

Chapter 5. This chapter draws conclusions and presents ideas for future work.

Appendices The appendices present (A) an independent check of Mach probe measurements using local spectroscopy measurements, (B) a study of probe perturbation of the plasma, and $(\mathrm{C})$ detailed analysis of helium ion emission lines for Doppler spectroscopy. 


\section{Chapter 2}

\section{Experimental Apparatus}

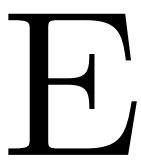

XPERIMENTAL results reported in this dissertation were obtained on the

Magnetic Reconnection Experiment (MRX) [YAmADA et al., 1997a], a laboratory facility located at the Princeton Plasma Physics Laboratory. MRX is a unique reconnection laboratory experiment in that 2-D reconnection layers can be formed quasi-statically (slow compared to the characteristic Alfvén time) and studied in plasmas which satisfy the MHD approximation globally. The ability to interpret, understand, and reproduce the experimental data requires detailed knowledge of the experimental apparatus and operation. Section 2.1 describes the vacuum hardware, magnetic field coils, and power system; Sec. 2.2 describes the plasma formation sequence and achievable plasma parameters; and Sec. 2.3 describes the diagnostic methods and data acquisition system.

\section{$2.1 \quad$ Experimental setup}

\subsubsection{Vacuum hardware}

A photograph of the vacuum chamber is shown in Fig. 2.1 and a side-view schematic is shown in Fig. 2.2. The vacuum chamber is made of $1 / 2$ in. stainless steel and has over fifty ports of various sizes for versatile diagnostic access. Several ports have windows for non-invasive optical diagnostics. Flanges are generally sealed with copper gaskets (Conflat). However, rubber O-rings (Wilson-seal or "quick connect") are also used on probe shafts, magnetic coil cable feed-thrus, and the two large end domes of the vacuum chamber. 


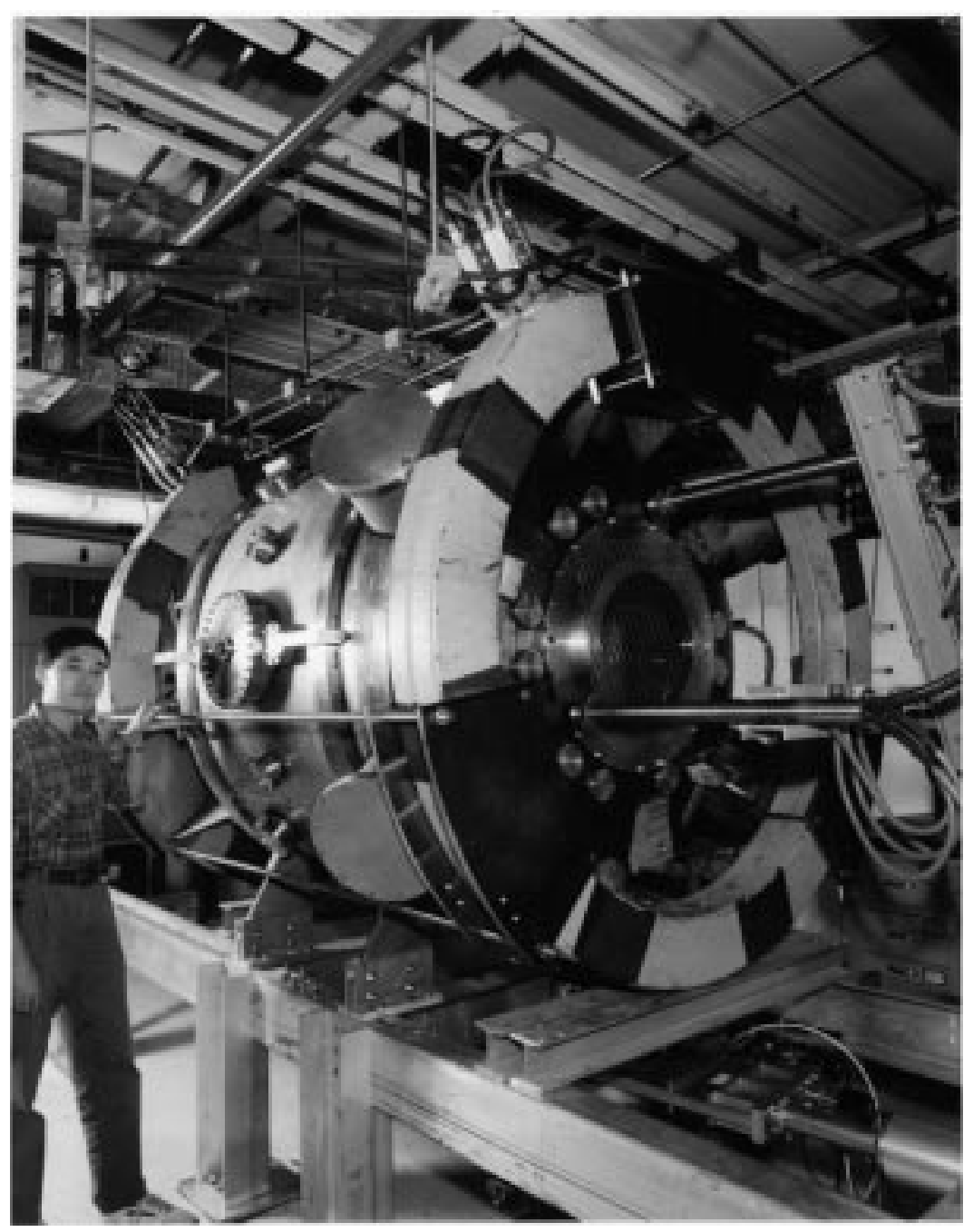

Figure 2.1: Photograph of MRX vacuum vessel with PPPL physicist Hantao Ji.

A base pressure of $1-2 \times 10^{-6}$ Torr is achieved with a turbomolecular pump. ${ }^{1} \mathrm{~A}$ cryo-pump ${ }^{2}$ is often used to reduce the base pressure further by $30-50 \%$. For initial pump-down, a mechanical forepump brings the base pressure to below 100 mTorr before the turbomolecular pump is engaged to continue pumping to the final base pressure. Fill gases, including $\mathrm{H}_{2}, \mathrm{D}_{2}, \mathrm{He}, \mathrm{Ne}$, and Ar, stored in compressed gas cylinders are introduced into the vacuum chamber via a electronically-gated gas valve. Stainless steel pipes with $3 / 8$ in. outer diameter (OD) are used to connect

\footnotetext{
${ }^{1}$ Pfeiffer model TPU-2200

${ }^{2}$ Varian Cryostack 8
} 


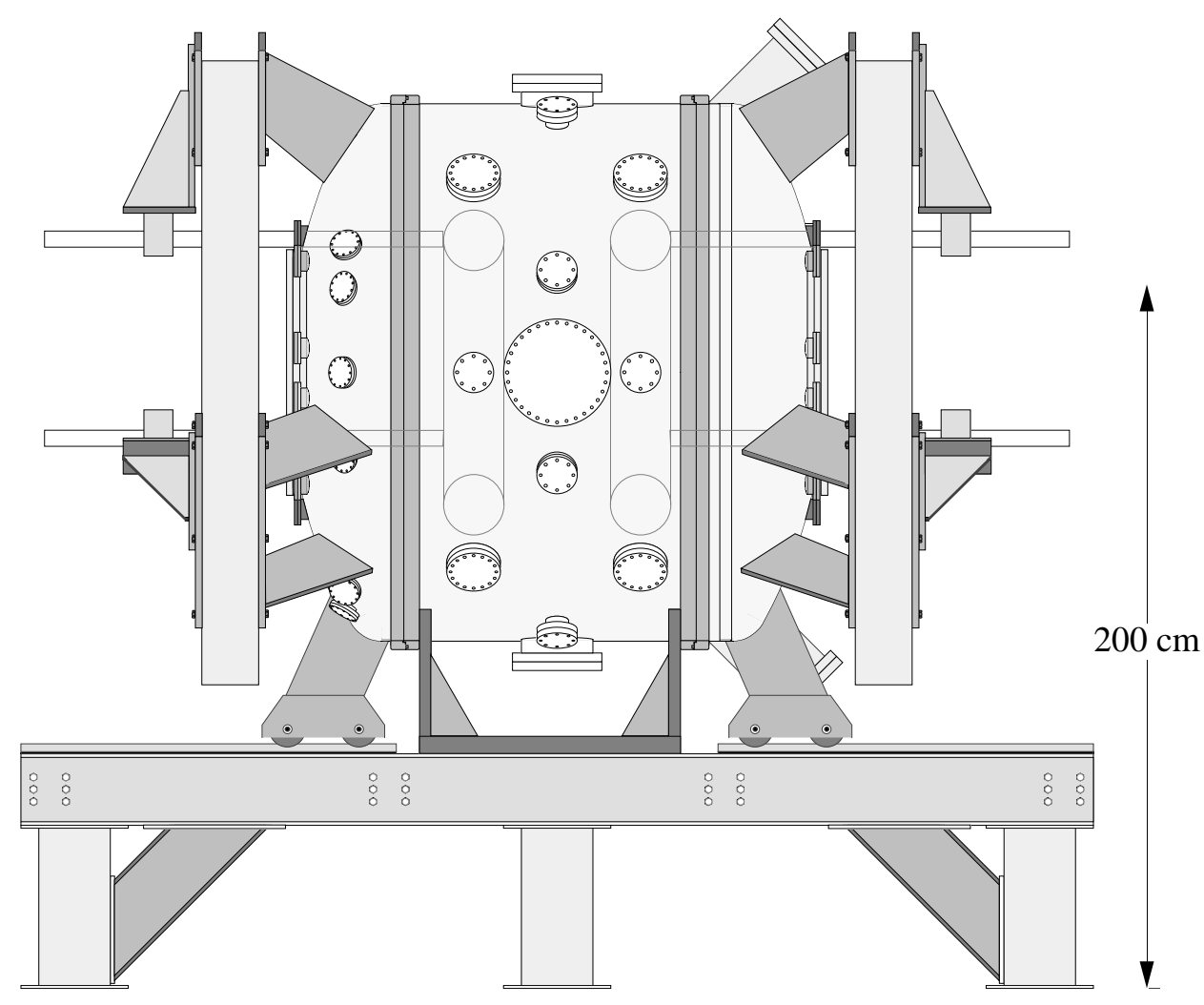

Figure 2.2: Side-view schematic of MRX vacuum vessel showing outlines of the internal flux-cores and the external equilibrium field coils, as well as the multiple diagnostic ports.

the regulated gas cylinders to the gas valve, which is attached directly to one port of the vacuum chamber. Typically, it takes approximately three seconds to introduce 2-12 mTorr of working gas into the chamber before the capacitor banks are discharged and the gas ionized (to be described later in this chapter). The base pressure is monitored with an ionization gauge and a residual gas analyzer (RGA), and the injected gas pressure is monitored with a 10 Torr (full-scale) Baratron gauge.

\subsubsection{Power system}

The main power system consists of two capacitor banks, charging and firing circuits, high current coaxial cables, and copper bus-bar interface to the coils. Each capacitor 
bank consists of eight $60 \mu \mathrm{F}$ oil-filled capacitors rated for $20 \mathrm{kV},{ }^{3}$ corresponding to a total stored energy of $96 \mathrm{~kJ}$ in each bank. High current switching is performed with Mercury-filled ignitrons, ${ }^{4}$ which are triggered electronically using delay timers and fiber optic links. This system is used to energize the pulsed internal magnetic field coils, to be described below. External coils which provide a DC shaping field, also described below, are energized by rectified AC power from a PPL power sub-station.

\subsubsection{Magnetic field coils}

\section{Flux-cores}

Two "flux-cores," which are the same type used in S-1 spheromak research [YAMADA et al., 1981], are used to generate the pulsed magnetic fields in MRX. The flux-cores are toroids with a major radius of $38 \mathrm{~cm}$ and a minor radius of $9 \mathrm{~cm}$ and are placed inside the vacuum vessel, each supported by three legs which also serve as feed-thrus for the coil currents; a side view of the setup is shown in Fig. 2.2. Inside each flux-core, there are two independent sets of coils: the poloidal field (PF) coil and the toroidal field (TF) coil. The PF coil consists of four 1-turn toroidal windings wound along the minor axis of the flux-core, and the toroidal field coil (TF) consists of two 18-turn solenoidal windings. The individual windings within each PF and TF coil can be connected in series or parallel, and likewise the two flux-cores can be connected in series or parallel. This flexibility allows different coil inductances and hence different discharge time scales and coil currents for the same capacitor voltage. The data reported in this dissertation were taken with everything connected in series except the two flux-cores in parallel. The inductances of each PF coil (four turns in series) and each TF coil (two 18-turn solenoids in series) were both measured to be approximately $30 \mu \mathrm{H}$.

The coils are insulated \#2 gauge copper braid wound onto a machined G10 core material. This internal structure is covered with fiberglass and then an aluminum shell, which has two toroidal and two poloidal gaps to allow field penetration. On top of the aluminum is a layer of polyester fiber, then wrapped fiberglass braid,

\footnotetext{
${ }^{3}$ Westinghouse style 5NA489981

${ }^{4}$ General Electric GL-37207A
} 
then two-component epoxy, ${ }^{5}$ and finally a coating of Inconel. ${ }^{6}$ The fiberglass braid prevents cracking on the layers above it, and the epoxy is intended to eliminate vacuum leaks, although some still remain and are differentially pumped through the flux core legs. The Inconel coating makes the flux core surface a conducting boundary, minimizing plasma-surface interactions, but at the same time gives it a relatively high resistivity, allowing for faster field penetration during the plasma formation stage.

The positions of the flux-cores along the symmetry axis can be adjusted. In all the experiments reported in this dissertation, the nearest surface-to-surface distance of the flux cores was $50 \mathrm{~cm}^{7}$

\section{Equilibrium field coils}

The equilibrium field $(\mathrm{EF})$ coils are two external coils (diameter $=64$ in.) arranged in a Helmholtz-like configuration. They each contain 28 turns of hollow copper conductors, which allow for water cooling. The resistance of each coil is approximately $30 \mathrm{~m} \Omega$. The EF coils are pulsed on for approximately $3 \mathrm{~s}$, during which the approximately $200 \mu$ s main plasma discharge occurs. Peak current is limited to $5 \mathrm{kA}$. Typical operational values are about $1.5 \mathrm{kA}$, which generates an on-axis magnetic field on the order of 200 Gauss. $^{8}$

\subsection{Plasma formation and achievable parameters}

\subsubsection{Plasma formation sequence}

MRX utilizes the flux-core plasma formation scheme [YAMADA et al., 1981] used on S-1, except that two smaller flux-cores are used instead of one larger flux-core. The coil currents in a typical discharge sequence, measured with Pearson current probes, are shown in Fig. 2.3. The formation sequence is as follows. PF coil

\footnotetext{
${ }^{5}$ Part\# EP21TCHT-1 from Master Bond, Inc., Hackensack, NJ

${ }^{6}$ Applied using an industrial electro-arc spray process, performed offsite at White Engineering Surfaces Corporation, Newtown, PA.

${ }^{7}$ Except for results in Fig. 4.2, which had a distance of $40 \mathrm{~cm}$.

${ }^{8}$ In June of 1998, a new set of EF coils with minor differences in dimension were installed. Other specifications are kept identical. The original EF coils are now installed on NSTX as PF coils.
} 


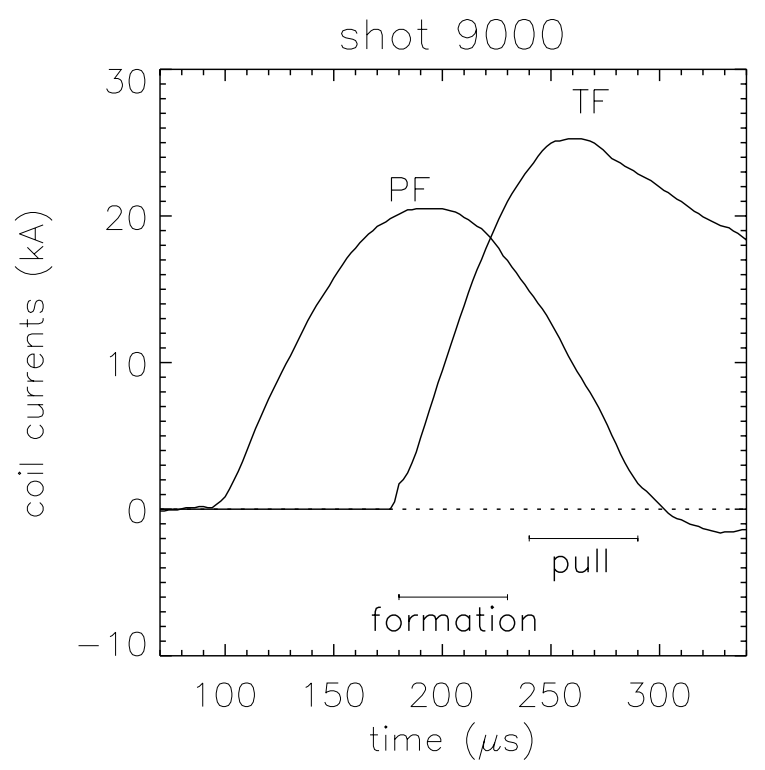

Figure 2.3: PF and TF coil current waveforms for a typical "pull" reconnection discharge. Plasma breakdown begins with initiation of the TF current at $t=180 \mu \mathrm{s}$. Pull reconnection occurs between approximately $t=240$ and $290 \mu \mathrm{s}$.

currents, initiated at $t=100 \mu \mathrm{s}(t=0$ is defined as the arbitrary time at which the firing circuit delay timers are first triggered), establish a quadrupole magnetic field as shown in Fig. 2.4(a). Shortly before the PF current peak is reached, the TF coil currents are initiated at $t=180 \mu$ s and generate a time-varying toroidal field inside the flux core. This in turn induces a poloidal inductive electric field around the flux-core. The inductive electric field ionizes the gas and creates a poloidal plasma current flowing around the flux-core. This plasma current generates a toroidal magnetic field outside the flux-core and within the plasma. The TF current keeps rising and therefore the toroidal field grows, creating a rotational transform and ultimately a toroidal plasma current. The sequence of events up to this point corresponds roughly to $t=230 \mu \mathrm{s}$ in Fig. 2.3. The TF current keeps rising strongly and net toroidal flux is injected into the "private" flux regions of each flux core and transferred into the "public" flux region, as indicated by the arrows in Fig. 2.4(b); this is "push" reconnection [YAMADA et al., 1997a]. When the TF current reaches its peak, the TF capacitor bank is shorted-out (or "crowbarred") by an ignitron at $t=270 \mu \mathrm{s}$, which allows the current in the TF circuit to damp away about its peak value instead of swinging back down to zero. At the same time, the PF 
(a)
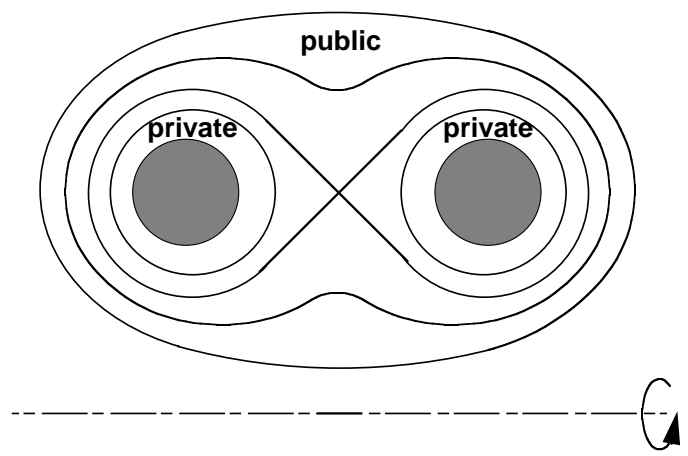

(b)

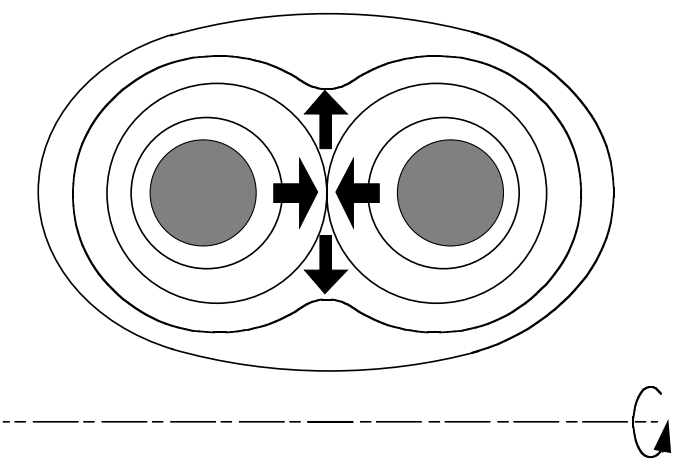

(c)

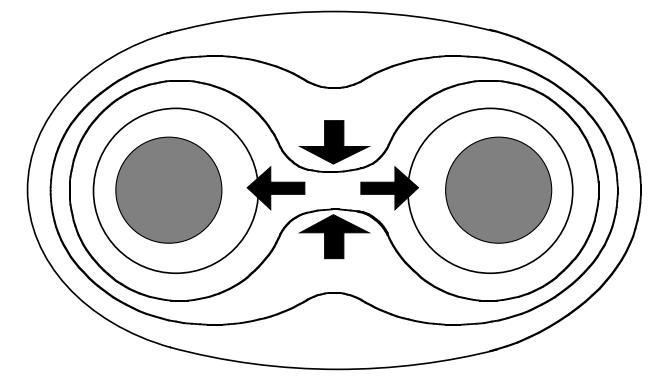

Figure 2.4: Shown schematically are cross-sectional views of the flux-cores with symmetry axis and poloidal flux contours at three different times. (a) Initial quadrupole field corresponding to time of peak PF current. "Public" and "private" flux regions are defined. (b) Formation stage of plasma during ramp-up of TF coil current and "push" reconnection. (c) TF current "crowbarred" and PF current ramps down, forming the "pull" reconnection layer. Note: distance between flux cores is fixed; the three drawings are not to scale. 


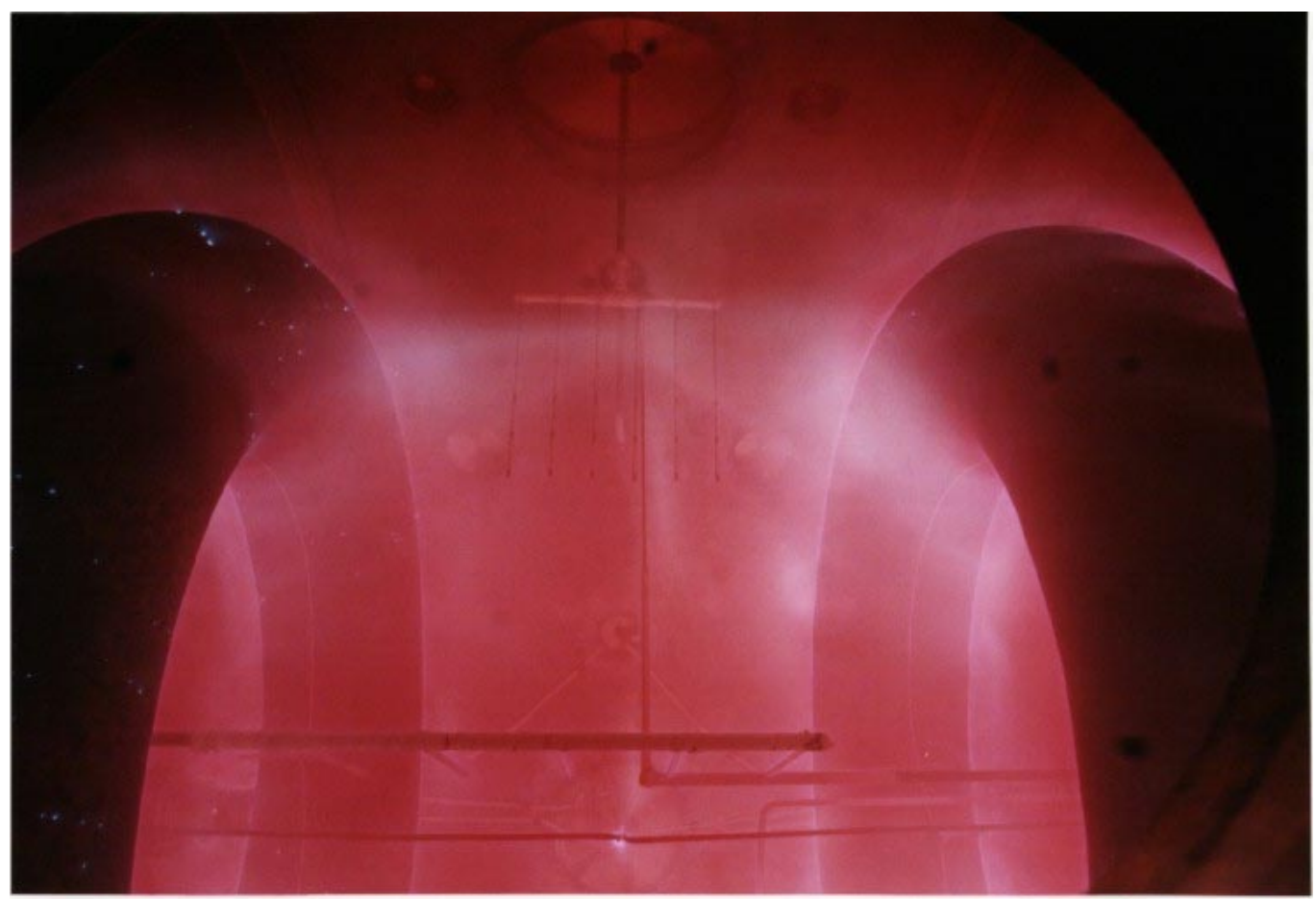

Figure 2.5: Visible-light photograph of the plasma during pull reconnection. The horizontally elongated current sheet coincides with the bright emission stretching across from one flux-core to the other. Many of the diagnostics are also in the field-of-view. Photograph, taken by D. Cylinder, first appeared in [Weiss, 1999]. 
current is ramping down strongly, and flux is "pulled" back from the public region into the private region of each flux-core (240-290 $\mu$ s in Fig. 2.3); this corresponds to "pull" reconnection as illustrated in Fig. 2.4(c). For about 20-30 $\mu \mathrm{s}$, which is approximately 15-20 Alfvén transit times, the "pull" reconnection layer is stable and quasi steady-state, allowing detailed study of the reconnection process. A visible-light photograph of the pull reconnection layer is shown in Fig. 2.5.

If the polarities of the two TF coils are equal, then the toroidal fields generated by the two flux-cores add, resulting in "co-helicity" reconnection in which field lines reconnect at a finite angle, as shown in Fig. 2.6(ii). If the polarities are opposite, then the fields generated reconnect at $180^{\circ}$, or exactly antiparallel. This case includes two sub-cases, "null-helicity" or "counter-helicity" reconnection, as shown in Figs. 2.6(i) and 2.6(iii), respectively. The "null-helicity" case is obtained only

(a)

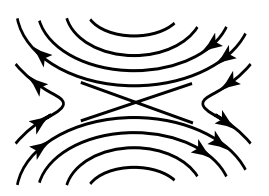

(b)

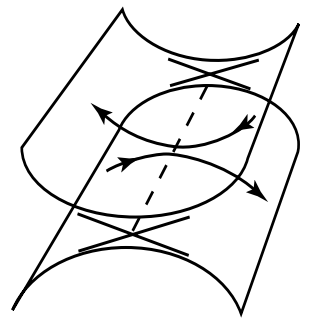

(i)

\begin{abstract}
Null-helicity (Antiparallel) Reconnection
\end{abstract}
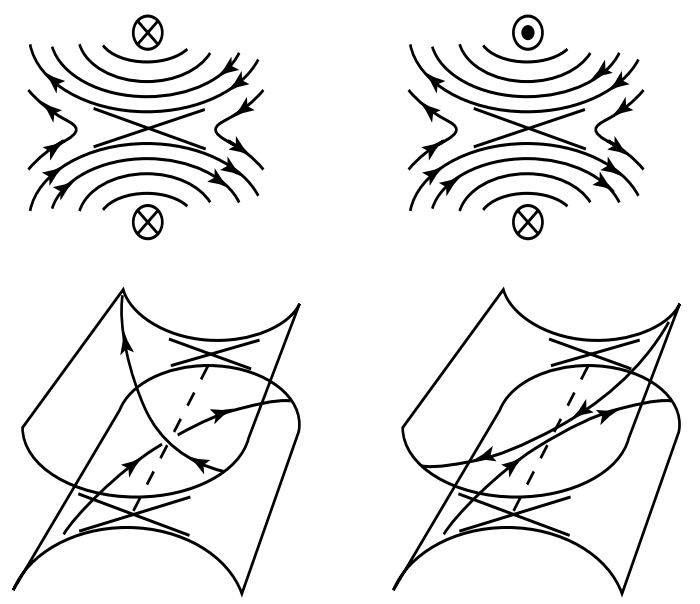

(ii)

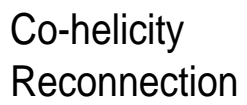

(iii )

Counter-helicity Reconnection

Figure 2.6: Illustration of three-component field line reconnection. (a) 2-D picture is independent of out-of-plane field, but (b) 3-D picture is clearly different. Out-ofplane magnetic fields in the merging plasmas are (i) zero for null-helicity, (ii) finite and add for co-helicity, and (iii) are finite and cancel for counter-helicity. Figure first appeared in [YAMADA et al., 1997b; YAMADA et al., 1997a].

during pull reconnection, in which public flux containing almost no toroidal field is reconnected into private flux. The "counter-helicity" case is obtained during push 
reconnection, ${ }^{9}$ in which private flux containing strong toroidal fields is reconnected into public flux.

The PF current is crowbarred at $t=290 \mu \mathrm{s}$ for pull reconnection. If the current were allowed to continue ramping down to large negative values, then the plasma would be "pinched-off" from the flux-cores and a spheromak or a field-reversed configuration (FRC) would be produced for the co-helicity or null-helicity cases, respectively. This scheme can be used to produce and study compact toroids, as proposed in the SPIRIT program [YAMADA et al., 1998]. If the PF current is crowbarred earlier, e.g. $t \approx 220 \mu \mathrm{s}$, then pull reconnection can be suppressed. This technique is utilized in Ch. 4 for correlating ion temperature rise with reconnection.

External coils provide a poloidal equilibrium field (EF). The coils are oriented to reduce the poloidal field generated by the PF coils, reducing it more strongly near the symmetry axis than away from the axis. Therefore, by adjusting the magnitude of the EF current, the radial force balance of the current sheet can be fine-tuned. The EF current is measured using a shunt resistor and input into a simple Fortran code to determine the magnetic field produced as a function of space. The accuracy of the code is checked with real measurements of the field at different spatial locations using a Gauss meter.

\subsubsection{Plasma parameters}

Plasma parameters are as follows: $n_{\mathrm{e}} \approx 2-15 \times 10^{13} \mathrm{~cm}^{-3}, T_{\mathrm{e}} \approx 5-20 \mathrm{eV}, T_{\mathrm{i}} \approx 5-$ $40 \mathrm{eV}$, and $B \approx 200-500$ Gauss. Relevant frequency and length scales are calculated and tabulated in Table 2.1 for typical hydrogen and helium discharges.

It is important to emphasize that the MHD criteria are satisfied globally in MRX, i.e. $\rho_{\mathrm{i}} \ll L, V_{\mathrm{A}} \ll c$, and $S \gg 1$. By varying the initial gas pressure, $n_{\mathrm{e}}$ and hence $\lambda_{\mathrm{mfp}, \mathrm{e}}$ can be varied over one order of magnitude, allowing the study of reconnection physics both in a collisional regime $\left(\lambda_{\mathrm{mfp}, \mathrm{e}} \approx \delta\right)$ and a collisionless regime $\left(\lambda_{\mathrm{mfp}, \mathrm{e}} \gg \delta\right)$. For hydrogen discharges, the classical energy equipartition time between electrons and ions ranges approximately from 20-80 $\mu$ s. For helium

\footnotetext{
${ }^{9}$ Presently, it is difficult to achieve a robust current sheet during push reconnection since the plasma formation phase and push phase overlap and use the same volt-seconds supplied by the capacitor bank. A third capacitor bank, which is currently being fabricated, would allow the push phase to be initiated after the formation phase is completed.
} 


\begin{tabular}{||c|c|c||}
\hline discharge & hydrogen & helium \\
\hline \hline$f_{\mathrm{pe}}(\mathrm{GHz})$ & 90 & 63 \\
\hline$f_{\mathrm{pi}}(\mathrm{GHz})$ & 2.1 & 0.7 \\
\hline$\Omega_{\mathrm{e}}(\mathrm{GHz})$ & 0.84 & 0.70 \\
\hline$\Omega_{\mathrm{i}}(\mathrm{MHz})$ & 0.46 & 0.10 \\
\hline$\nu_{\mathrm{ee}} \sim \nu_{\mathrm{ei}}(\mathrm{MHz})$ & 101 & 28 \\
\hline$\nu_{\mathrm{ii}}(\mathrm{MHz})$ & 1.7 & 0.2 \\
\hline$\nu_{\mathrm{ie}}(\mathrm{kHz})$ & 39.7 & 2.3 \\
\hline$\rho_{\mathrm{i}}(\mathrm{cm})$ & 1.9 & 3.2 \\
\hline$\rho_{\mathrm{e}}(\mathrm{mm})$ & 0.25 & 0.37 \\
\hline$c / \omega_{\mathrm{pi}}(\mathrm{cm})$ & 2.3 & 6.4 \\
\hline$\lambda_{\mathrm{mfp}, \mathrm{e}}(\mathrm{cm})$ & 1.3 & 5.9 \\
\hline$\lambda_{\mathrm{mfp}, \mathrm{i}}(\mathrm{cm})$ & 3.2 & 9.5 \\
\hline$\delta(\mathrm{cm})$ & 1.5 & 3 \\
\hline$L(\mathrm{~cm})$ & 10 & $5-10$ \\
\hline$\tau_{\mathrm{A}}(\mu \mathrm{s})$ & 1.5 & 2.6 \\
\hline$S\left(\mathrm{using} \eta_{\mathrm{Sp}}\right)$ & 228 & 185 \\
\hline
\end{tabular}

Table 2.1: Relevant frequency and length scales in MRX plasmas. For typical hydrogen discharges: peak $n_{\mathrm{e}}=10^{14} \mathrm{~cm}^{-3}, B=300 \mathrm{G}, T_{\mathrm{e}}=10 \mathrm{eV}$, and $T_{\mathrm{i}}=$ $30 \mathrm{eV}$, and for typical helium discharges: $n_{\mathrm{e}}=5 \times 10^{13} \mathrm{~cm}^{-3}, B=250 \mathrm{G}$, and $T_{\mathrm{e}} \approx T_{\mathrm{i}}=15 \mathrm{eV}$. Electron-neutral collisions are estimated to be negligible; however, their effects must be characterized in more detail.

discharges, this value increases to more than $200 \mu \mathrm{s}$, due to the difference in ion mass and also higher $T_{\mathrm{e}}$. Therefore, classical energy exchange between electrons and ions during the reconnection process $\left(\tau_{\text {rec }} \approx 20-30 \mu \mathrm{s}\right)$ is a small effect in hydrogen discharges and can be ignored completely in helium discharges.

\section{Electron-neutral collisions}

It is also important to characterize the effect of electron-neutral collisions. In hydrogen, $\tau_{\mathrm{ei}} \sim 10 \mathrm{~ns}\left(T_{\mathrm{e}}=10 \mathrm{eV}\right)$. The total reaction rate coefficient for electron interactions with ground state hydrogen neutrals is $\langle\sigma v\rangle \approx 1.6 \times 10^{-8} \mathrm{~cm}^{3} / \mathrm{s}$. ${ }^{10}$ If

\footnotetext{
${ }^{10}$ The reaction rate coefficients are taken from [JANEV et al., 1987].
} 
the neutral density is taken to be equal to $n_{\mathrm{e}} \approx 1 \times 10^{14} \mathrm{~cm}^{-3}$ (an extreme upper bound), then $\tau_{\text {en }} \sim 60 \tau_{\text {ei }} \sim 0.6 \mu$ s, indicating that electron-neutral collisions are a negligible effect. The reaction rate coefficient can be a factor of 100 larger for electron collisions with excited hydrogen neutrals, but the density of the excited neutrals should be very small since they are quickly ionized. Diatomic hydrogen also exists, and the sum reaction rate coefficient for all electron collisions with non-excited $\mathrm{H}_{2}$ is $\langle\sigma v\rangle \approx 2.1 \times 10^{-8} \mathrm{~cm}^{3} / \mathrm{s}$, resulting in a collision time (also assuming a neutral density equal to $n_{\mathrm{e}}=1 \times 10^{14} \mathrm{~cm}^{-3}$ ) of $50 \tau_{\mathrm{ei}} \sim 0.5 \mu \mathrm{s}$. Thus, these interactions are also negligible. In helium, $\tau_{\mathrm{ei}} \sim 35 \mathrm{~ns}\left(T_{\mathrm{e}}=15 \mathrm{eV}\right)$. The total reaction rate coefficient for electron interactions with ground state helium neutrals is $\langle\sigma v\rangle \approx 4 \times 10^{-9} \mathrm{~cm}^{3} / \mathrm{s}$. If the neutral density is taken to be equal to $n_{\mathrm{e}} \approx\left(5 \times 10^{13} \mathrm{~cm}^{-3}\right.$, an extreme upper bound $)$, then $\tau_{\mathrm{en}} \sim 142 \tau_{\mathrm{ei}} \sim 5 \mu \mathrm{s}$, indicating that electron-neutral collisions are a negligible effect in helium also. Extreme overestimates were used for the neutral density in arriving at these answers, and thus the conclusion that electron-neutral effects are negligible appears to be reasonable. Nevertheless, a more detailed calculation using computer codes is warranted to fully characterize the effect of electron-neutral collisions.

\subsection{Diagnostics}

Choices for diagnostics have been driven mainly by two concerns: (1) the need for local measurements with good time resolution $\left(\ll \tau_{\text {rec }}\right)$ and spatial coverage (over a $20 \times 60 \mathrm{~cm}$ area) and (2) a limited budget. Due to the relatively low temperatures $(<20 \mathrm{eV})$ and short pulse-lengths $(<300 \mu \mathrm{s})$ of MRX discharges, insertable magnetic and electrostatic probes, ${ }^{11}$ which satisfy both critera above, can be used routinely. Additionally, the availability of a spectrometer, CCD camera, and novel spectroscopy probe made the local ion temperature measurements possible.

\footnotetext{
${ }^{11}$ All designed and constructed by David Cylinder.
} 


\subsubsection{Magnetic probe arrays}

Local magnetic field measurements are obtained using arrays of miniature pickup coils. The time rate of change of magnetic flux through a small coil can be determined by measuring the voltage $V_{\text {coil }}$ across the two leads of the coil. If the size of the coil is very small compared to spatial variations of the magnetic field, Faraday's law yields

$$
V_{\text {coil }}=-\frac{\partial \Phi}{\partial t} \approx-N A \frac{\partial \hat{B}}{\partial t}
$$

where $\Phi$ is the total flux through the coil, $N$ is the number of turns in the coil, $A$ is the area of the coil, and $\hat{B}$ the average time-varying magnetic field passing through the coil. Integrating the voltage electronically then yields the magnitude of $\hat{B}$ at the coil. ${ }^{12}$

Throughout most of the experimental operation, three probe arrays were used, as shown in Fig. 2.7: (1) a 2-D $6 \times 5$ array with a $4 \mathrm{~cm}$ square grid and 3 orthogonally oriented probes at each grid point (henceforth, called "90-channel probe"), (2) a 2-D $5 \times 4$ array with $6 \mathrm{~cm} R$ resolution and $8 \mathrm{~cm} Z$ resolution and also 3 probes at each grid point (henceforth, called "60-channel probe"), and (3) a high-resolution 29 channel 1-D array with $0.5 \mathrm{~cm}$ resolution (1 probe at each position). The two 2-D probes are located $90^{\circ}$ apart in the $\theta$ direction. The 29 -channel probe can be rotated in the $\theta$ direction but it's default position is near the toroidal location of the 90channel probe. Also shown in Fig. 2.7 are the placement of flux loops and Rogowski coils, which measure total magnetic flux and plasma current, respectively, ${ }^{13}$ and the cylindrical coordinate system $(R, Z, \theta)$ used throughout this dissertation.

Probe pickup coils are each wound with eighty turns of \#38 gauge insulated copper wire on custom-made spools with alumina $\left(\mathrm{Al}_{2} \mathrm{O}_{3}\right)$ cores and mylar ends. The coil spools have length and diameter of $3 \mathrm{~mm}$. They are aligned precisely and glued onto fiberglass strips with the help of a pre-machined jig and then slid down $5 \mathrm{~mm}$ O.D. glass tubes. The glass tubes of 2-D probe arrays are oriented with respect to each other using another pre-machined jig, and then all the glass tubes are attached to a $3 / 8$ in. stainless steel shaft using epoxy. The spatial orientation

\footnotetext{
${ }^{12}$ See $e . g$. [LOVBERG, 1965] for a complete account of magnetic probes.

${ }^{13}$ See e.g. [HUTChInson, 1987] for details.
} 


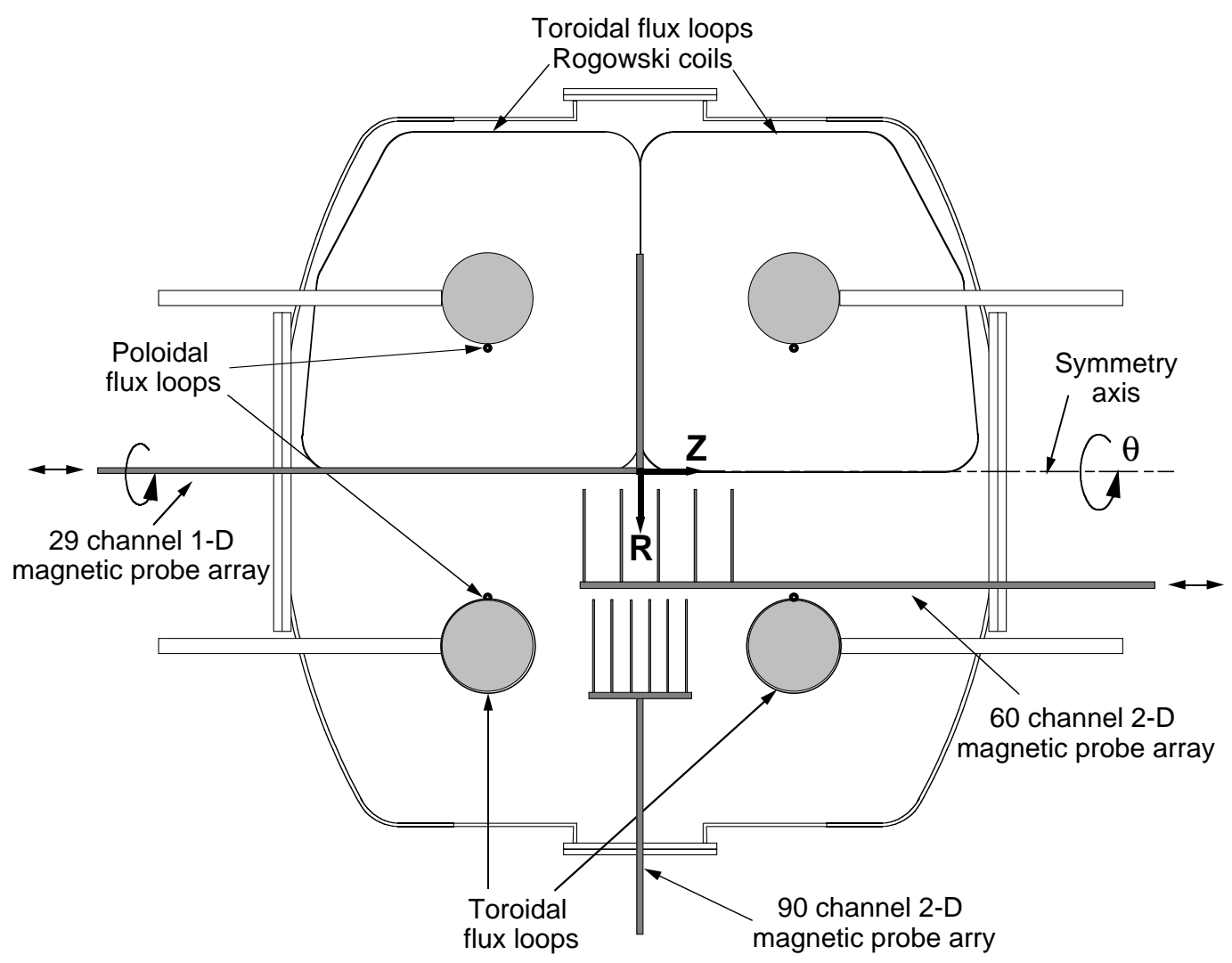

Figure 2.7: Setup of magnetic probe arrays, flux loops, and Rogowski coils. Note the definition of the cylindrical coordinate system, which is used throughout this work.

of each individual probe in the final assemblies should be better than a few degrees. A photograph of one of the arrays, the high-resolution 1-D probe array, is shown in Fig. 2.8. Approximately $1.5 \mathrm{~m}$ lengths of the \#38 wire are left connected to both ends of each spool. They are arranged in twisted pairs (to minimize inductance) and then brought out of the vacuum chamber inside the supporting stainless steel shaft. From there, they are connected to RG-174/U cables for a $35 \mathrm{ft}$. run to the integrators in the control room next door. The RG-174/U cables are bundled inside a grounded aluminum conduit to shield out electrostatic noise.

Before installing probe arrays into the vacuum vessel, the $N A\left(\sim 1.5 \mathrm{~cm}^{2}\right)$ of each individual pickup coil was determined (with $2 \%$ measurement error) by (1) placing each probe into the known, spatially uniform field $B$ of a Helmholtz coil driven with a sinusoidal current $(f=10 \mathrm{kHz}),(2)$ measuring the peak coil voltage 


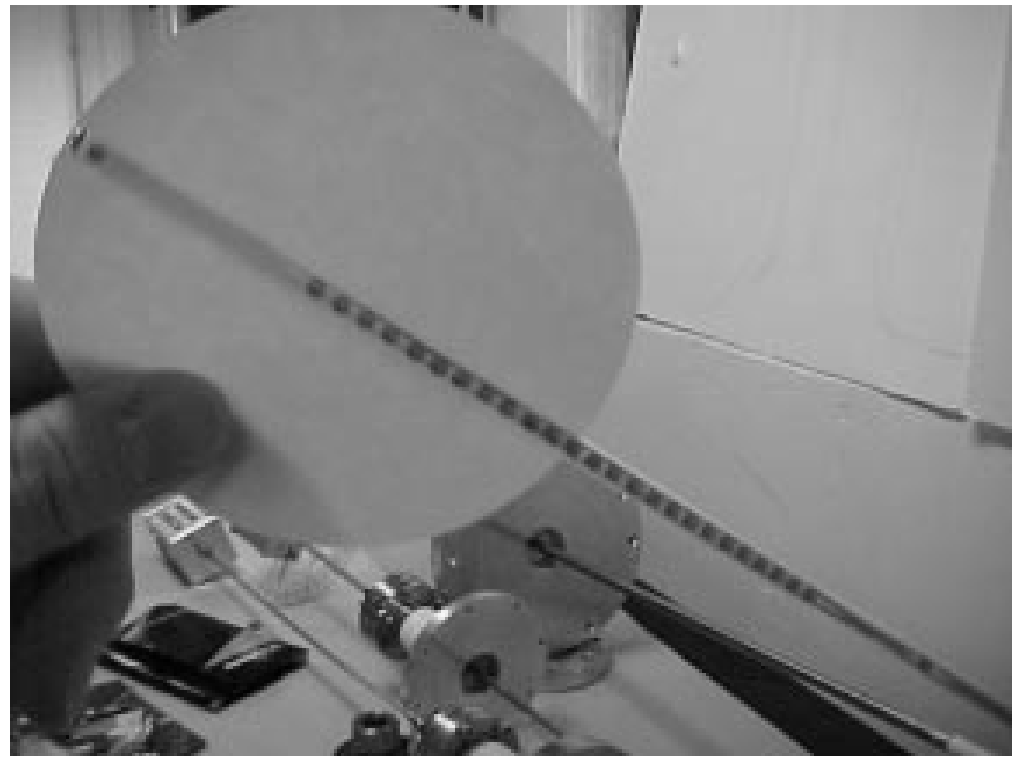

Figure 2.8: Photograph of the high-resolution 1-D magnetic probe array. The glass tube has an OD of $5 \mathrm{~mm}$. The pick-up coils inside are spaced by $5 \mathrm{~mm}$ and are oriented to measure the reconnecting field component.

$V_{\max }$ on an oscilloscope, and (3) using Eq. (2.1) which becomes

$$
N A=\frac{V_{\max }}{2 \pi f \hat{B}_{\max }} .
$$

Coil voltages are integrated electronically using home-made active integrators with effective time constant $R_{\mathrm{i}} C_{\mathrm{i}}(\sim 100 \mu \mathrm{s})$, which are each determined (also with $2 \%$ measurement error) by inputting a sinusoidal signal $V_{\mathrm{i}}(f=1 \mathrm{kHz})$ from a function generator into the integrator and measuring the output $V_{\mathrm{o}}$,

$$
R_{\mathrm{i}} C_{\mathrm{i}}=\frac{V_{\mathrm{i}, \max }}{V_{\mathrm{o}, \max }} \frac{1}{2 \pi f}
$$

The time-varying magnetic field $\hat{B}$ is then

$$
\hat{B}=\frac{R_{\mathrm{i}} C_{\mathrm{i}}}{N A} V_{\mathrm{o}}
$$

Probe sensitivity and frequency response are determined by $N A$ and $R_{\mathrm{i}} C_{\mathrm{i}}$ values. 
The probe sensitivity is

$$
\frac{V_{\mathrm{o}}}{\hat{B}}=\frac{N A}{R_{\mathrm{i}} C_{\mathrm{i}}} \approx \frac{1.5 \mathrm{~cm}^{2}}{100 \mu \mathrm{s}} \approx 150 \mathrm{mV} / \mathrm{kG}
$$

which, for typical peak $\hat{B} \sim 500 \mathrm{G}$, results in an easily detectable $75 \mathrm{mV}$ signal. The probe signals are digitized with a bit resolution of approximately $5 \mathrm{G}$. The probe frequency response is limited by the inductance $L$ of the coil and the resistance $R$ seen by the coil. The shortest time scale which can be resolved by the coils is

$$
\tau=\frac{L}{R} \approx \frac{10 \mu \mathrm{H}}{20 \mathrm{k} \Omega} \approx 0.5 \mathrm{~ns}
$$

which is well below the necessary time resolution $(\sim 5 \mu \mathrm{s})$. In fact, the frequency response is limited to a few $\mathrm{MHz}$ by the $\mathrm{RG}-174 / \mathrm{U}$ cable and the filtering effect of the integrators. ${ }^{14}$

The total field $B$ is the sum of the time-varying field $\hat{B}$ and the corresponding component of the DC EF field $B_{\mathrm{EF}}$, which is not detected by the magnetic probes. A computer code is used to calculate all the components of the EF field, which is then subtracted from the result in Eq. (2.4) at each probe location to arrive at the final result for $B$ used in subsequent data analysis,

$$
B(t)=\hat{B}(t)-B_{\mathrm{EF}}=\frac{R_{\mathrm{i}} C_{\mathrm{i}}}{N A} V_{\mathrm{o}}(t)-B_{\mathrm{EF}}
$$

The relative error in $B$ arises from both the measurement error of $R_{\mathrm{i}} C_{\mathrm{i}}$ and $N A$ and the bit resolution of the digitized signal. For large values of $B$, the relative error is less than $5 \%$. As $B$ gets small near the reconnection layer, the relative error can become large due to the bit resolution; however, in these instances the absolute error is still limited to $5 \mathrm{G}$ and should not affect any of the data interpretation. To illustrate the comprehensiveness of the magnetic probe measurements, a surface plot of the 90-channel probe array measurement of the reconnecting field $B_{\mathrm{Z}}$ is shown in Fig. 2.9.

\footnotetext{
${ }^{14}$ Measurement of high-frequency fluctuations (> $1 \mathrm{MHz}$ ), perhaps driven by instabilities in the reconnection region, would require further, careful consideration of the frequency responses of all components along the signal path and the identification and reduction of spurious high frequency noise in the power system [CARTER et al., 1999]. It would also require the use of electrostatic probes to be described in the next section.
} 


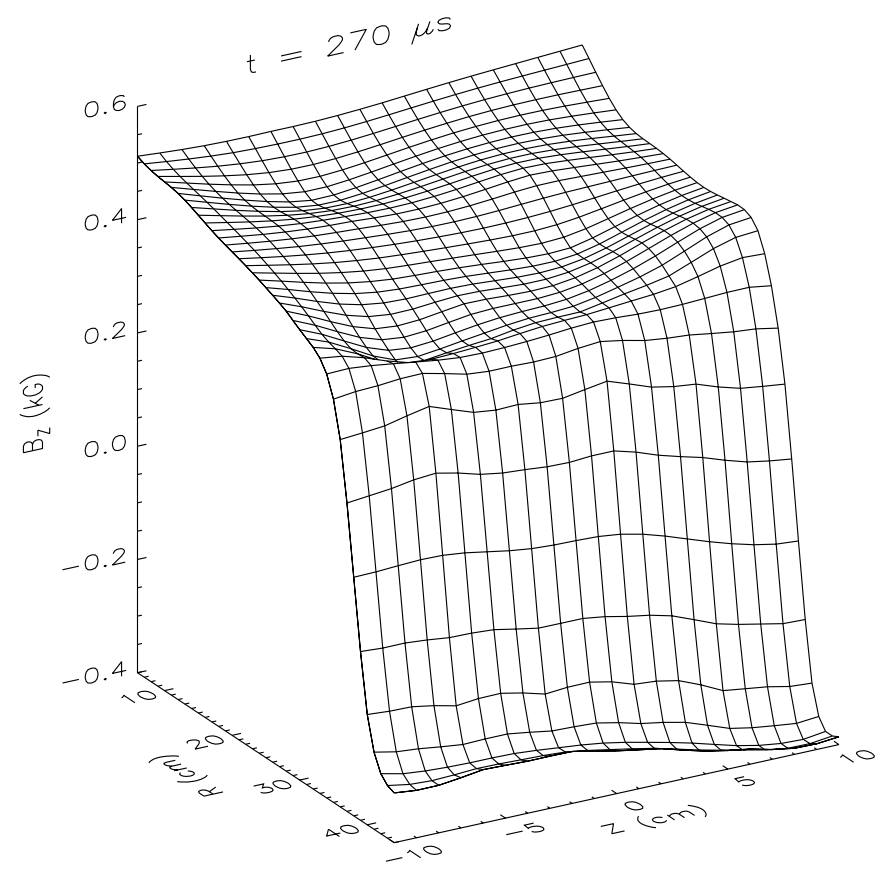

Figure 2.9: Surface plot of the reconnecting field $B_{\mathrm{Z}}$ as measured by the 90-channel probe array. Interpolation from the $4 \mathrm{~cm}$ by $4 \mathrm{~cm}$ probe grid to the $1 \mathrm{~cm}$ by $1 \mathrm{~cm}$ data set shown above is done numerically.

\subsubsection{Electrostatic probes}

One triple Langmuir probe and two Mach probes are used routinely to measure plasma density, electron temperature, and ion flow speeds. Placement of the probes in the vacuum vessel is shown in Fig. 2.10.

\section{Triple Langmuir probe}

The triple Langmuir probe [Chen and Sekiguchi, 1965] is a standard diagnostic used in many laboratory plasma experiments. It yields measurements of instantaneous temperature $T_{\mathrm{e}}$ and ion saturation current $I_{\text {sat }}$ without requiring a voltage sweep, which is necessary for single and double Langmuir probe measurements. ${ }^{15}$

A schematic of the triple probe bias circuit is shown in Fig. 2.11. As is evident in the figure, the triple probe is a combination of a double probe (biased to collect

\footnotetext{
${ }^{15}$ See e.g. [Chen, 1965; HutCHINSON, 1987] for a general account of single and double Langmuir probe theory.
} 


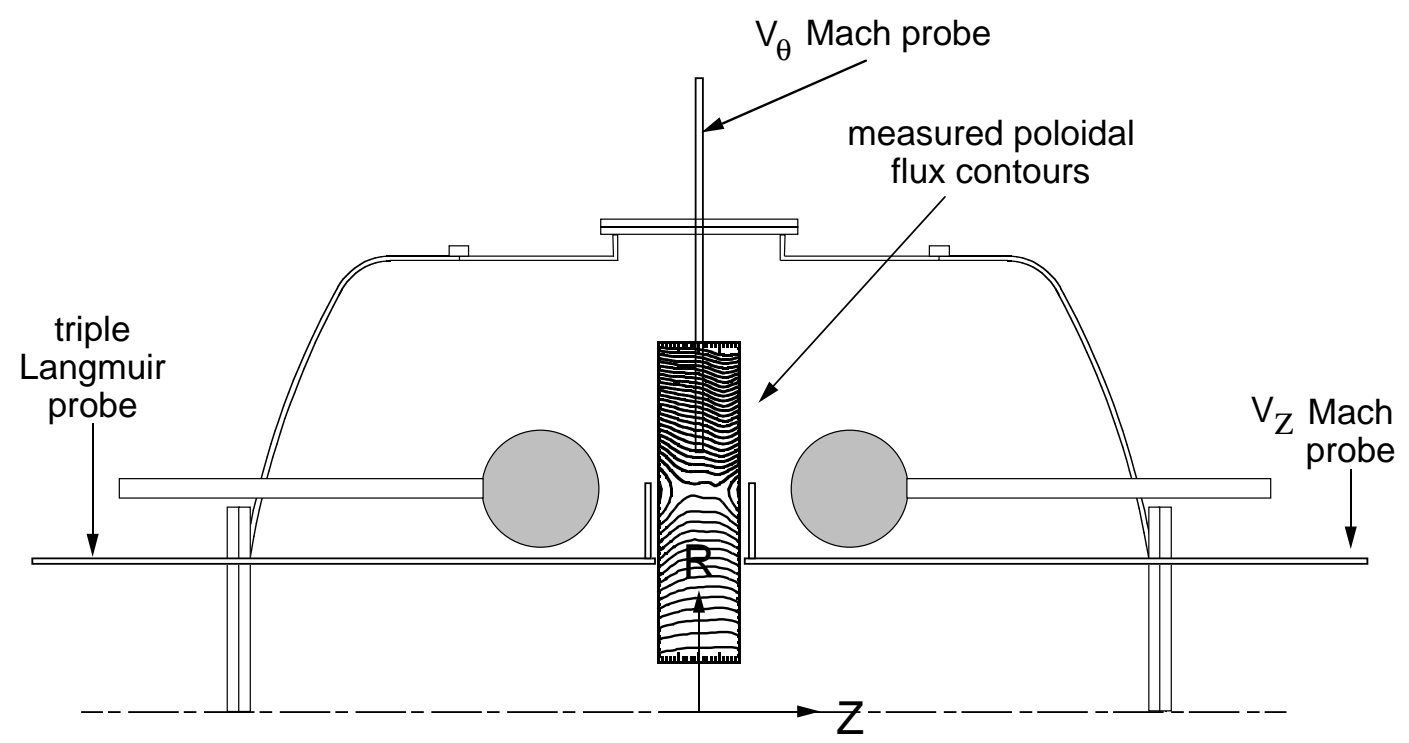

Figure 2.10: Setup of triple Langmuir and Mach probes. The triple Langmuir and $V_{\mathrm{Z}}$ Mach probe can be scanned in $R$ and $Z$, and the $V_{\theta}$ Mach probe can only be scanned in $R$.

$I_{\text {sat }}$ ) and a floating single probe. The voltage $V_{+\mathrm{f}}$ (as a function of time) between the positively biased electrode of the double probe and the floating probe is measured with one channel of an attenuating optical isolator unit ${ }^{16}$ (10 M $\Omega$ input impedance). A relationship between $V_{+\mathrm{f}}$ and $T_{\mathrm{e}}$ can be derived by writing down equations for the currents and voltages at each probe tip, and using the fact that the ion current is $I_{\text {sat }}$ and the electron current is governed by the Boltzmann factor $\exp \left[\left(V-V_{\mathrm{p}}\right) / T_{\mathrm{e}}\right]$, where $V_{\mathrm{p}}$ is the plasma potential and $T_{\mathrm{e}}$ is in $\mathrm{eV}$ units. Combining the equations yields $^{17}$

$$
\frac{1}{2}=\frac{1-\exp \left(-V_{+\mathrm{f}} / T_{\mathrm{e}}\right)}{1-\exp \left(-V_{\text {bias }} / T_{\mathrm{e}}\right)}
$$

\footnotetext{
${ }^{16}$ Tektronix model A6907

${ }^{17}$ See [Chen and SeKiguchi, 1965; Ji et al., 1991] for a thorough development of the triple probe theory.
} 


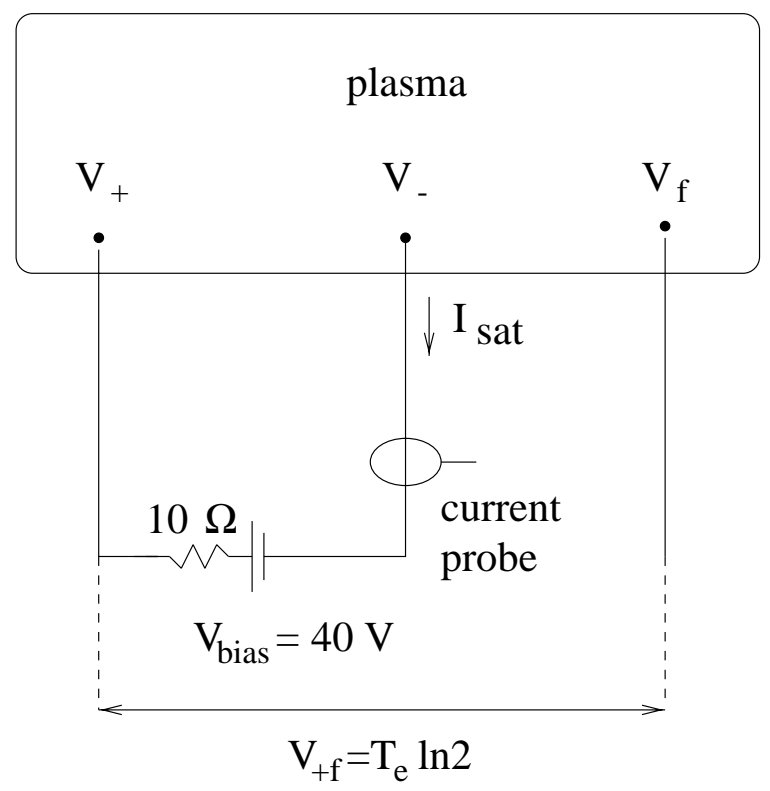

Figure 2.11: Schematic of triple Langmuir probe biasing scheme.

which, in the limit $V_{\text {bias }} \gg T_{\mathrm{e}}$ simplifies to

$$
T_{\mathrm{e}}=\frac{V_{+\mathrm{f}}}{\ln 2} \approx 1.44 V_{+\mathrm{f}}
$$

Typically in the experiments, $V_{\text {bias }}=40 \mathrm{~V}$ and $T_{\mathrm{e}} \sim 10-20 \mathrm{eV}$, and thus the approximation made in Eq. (2.9) is correct to better than $2 \%$. The floating potential $V_{\mathrm{f}}$ is generally around $-100 \mathrm{~V}$ during pull, null-helicity reconnection and $+50 \mathrm{~V}$ for pull, co-helicity reconnection (both with respect to machine ground). ${ }^{18}$ The bias voltage is applied by charging up an $82 \mu \mathrm{F}$ electrolytic capacitor (with $0.1 \mu \mathrm{F}$ and $0.01 \mu \mathrm{F}$ bypass ceramic capacitors in parallel $)^{19}$ using a DC power supply. Thus, $V_{\text {bias }}$ can be varied arbitrarily from shot-to-shot. The power supply is disconnected from the capacitor prior to the plasma discharge so the entire probe circuit has no DC path to ground.

For confirmation of the $T_{\mathrm{e}}$ measurement, an $I-V$ characteristic using the wellknown double probe method $^{20}$ was obtained whenever a new probe was installed, a

\footnotetext{
${ }^{18}$ This is an interesting result which is admittedly not understood at present. It clearly has implications for ion and electron losses in the two configurations.

${ }^{19}$ Bypass capacitors are used to supply charge on short time scales so that the bias voltage can be maintained even in the presence of fast spikes in the probe potential.

${ }^{20}$ See e.g. [Hutchinson, 1987].
} 
new fill gas used, or a new machine condition encountered (e.g. after re-coating the flux cores with Inconel). Generally, the $T_{\mathrm{e}}$ obtained using the double probe $I-V$ characteristic was seen to agree with the triple probe value to better than $20 \%$.

The time-resolved ion saturation current $I_{\text {sat }}(t)$, typically on the order of $500 \mathrm{~mA}$ (probe area is given below), is measured by placing a $1 \mathrm{~V} / \mathrm{A}$ current monitor ${ }^{21}$ on the double probe current path. Bohm's sheath criteria [Bонм et al., 1949] gives, for $T_{\mathrm{i}} \ll T_{\mathrm{e}}$,

$$
I_{\text {sat }}=\exp (-1 / 2) A e n_{\mathrm{i}} \sqrt{\frac{T_{\mathrm{e}}}{m_{\mathrm{i}}}},
$$

from which the ion density $n_{\mathrm{i}}$ can be deduced. In MRX, however, $T_{\mathrm{i}} \sim T_{\mathrm{e}}$ in helium discharges, in which $T_{\mathrm{i}}$ can be measured rigorously via spectroscopy (see Sec. 2.3.3). Exact numerical solutions of $I_{\text {sat }}$ for $T_{\mathrm{i}} / T_{\mathrm{e}}=0 \rightarrow 1$ are given in [LAFRAMBoise, 1966], which shows that $I_{\text {sat }}$ varies by only a few percent as $T_{\mathrm{i}} / T_{\mathrm{e}}$ is varied from 0 to 1. Therefore, Eq. (2.10) can be used to determine $n_{\mathrm{i}}$ and $n_{\mathrm{e}}$, the latter by invoking quasi-neutrality.

In the experiments, there is some evidence that $T_{\mathrm{i}} \gtrsim 2 T_{\mathrm{e}}$ for hydrogen discharges, although these results are not rigorous and are not emphasized in the dissertation. In this case, Eq. (2.10) does not apply, and there is no generally accepted theory for $I_{\text {sat }}$. However, a commonly used modified expression for $T_{\mathrm{i}} \gtrsim T_{\mathrm{e}}$ is [STANGEBY, $1984 \mathrm{~b}]$

$$
I_{\mathrm{sat}}=\exp (-1 / 2) \operatorname{Aen}_{\mathrm{i}} \sqrt{\frac{T_{\mathrm{e}}+T_{\mathrm{i}}}{m_{\mathrm{i}}}}
$$

Since $T_{\mathrm{i}}$ is not known precisely in hydrogen discharges, values of density are calibrated with an independent measurement using a second-harmonic interferometer system [BRETz et al., 1997], which gives an absolute measurement of the line-integrated density. By comparing the triple Langmuir probe $n_{\mathrm{e}}$ profile using Eq. (2.10) with the absolute line-integrated value, it was determined that $n_{\mathrm{e}}$ calculated using Eq. (2.10) was a factor of two too small. This, however, is within the generally expected accuracy of Langmuir probe density measurements. An empirical calibration factor of two was used in determining $n_{\mathrm{e}}$ for hydrogen discharges. In

\footnotetext{
${ }^{21}$ Pearson Electronics model 2877
} 


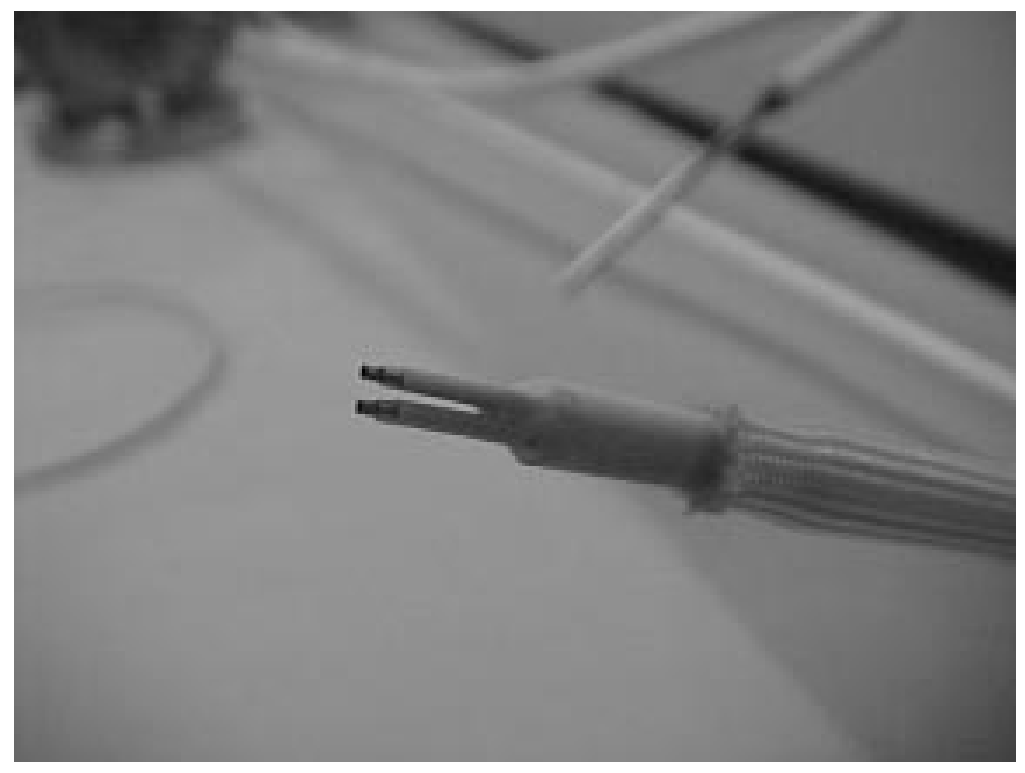

Figure 2.12: Photograph of the triple Langmuir probe, with a fourth tip for backup. The tungsten tips are spaced apart by $4 \mathrm{~mm}$ and held in place by alumina rods.

helium, pressure balance, i.e. $n\left(T_{\mathrm{i}}+T_{\mathrm{e}}\right)$ in the reconnection layer is equal to $B^{2} / 2 \mu_{0}$ outside the layer, indicates that the triple probe $n_{\mathrm{e}}$ measurement is approximately correct.

The MRX triple probe, shown in Fig. 2.12, consists of a 1/4 in. OD stainless steel shaft, attached to the end of which is a piece of cylindrical alumina which is $0.5 \mathrm{~cm}$ in diameter and $2.3 \mathrm{~cm}$ long. The alumina houses four smaller alumina rods, which in turn each house a 30 mil diameter tungsten rod. The small alumina rods extend beyond the big alumina rod by $1.25 \mathrm{~cm}$, and the tungsten rods extend beyond the small alumina rods by $1 \mathrm{~mm}$. The area of each probe tip is $2.85 \times 10^{-2} \mathrm{~cm}^{2}$. The tips are spaced $2 \mathrm{~mm}$ apart in a square configuration. Four twisted leads, which run along the inside of the stainless steel shaft, provide electrical connections to the four tungsten rods. The stray capacitance between leads and from each lead to machine ground is on the order of $150 \mathrm{pF}$, which is negligible for the (non-fluctuating) time scale of interest $(5-10 \mu \mathrm{s})$.

\section{Mach probe}

Local ion flow speed $V_{\mathrm{i}}$ is measured by a Mach probe, which collects ion saturation current $I_{\text {sat }}$ [Eq. (2.10)] on each of two oppositely facing electrodes. Intuitively, it is 
reasonable to expect that the difference between the collected currents should reveal information about the average ion drift speed past the probe. The experimental quantity measured is

$$
K \equiv \frac{I_{+}-I_{-}}{I_{+}+I_{-}}
$$

where $I_{+}$and $I_{-}$are the (ion saturation) currents collected by the upstream and downstream electrodes, respectively. By considering the appropriate probe sheath physics, a theoretical relationship between $K$ and $V_{\mathrm{i}}$ can be postulated.

A large number of Mach probe papers exist in the literature, e.g. [HuDIs and Lidsky, 1970; Stangeby, 1984a; Chung, 1990; Peterson et al., 1994], and a review of them will not be attempted here. The important parameters to consider are $a / \lambda_{\mathrm{D}}, T_{\mathrm{i}} / T_{\mathrm{e}}$, and $\rho_{\mathrm{i}} / a$, where $a$ is the characteristic probe electrode dimension. MRX plasma and Mach probe parameters fall into the regime $a / \lambda_{\mathrm{D}} \gg 1, T_{\mathrm{i}} \gtrsim T_{\mathrm{e}}$, and $\rho_{\mathrm{i}} / a \gg 1$, in which there is no general, rigorous theory to predict $I_{\text {sat }}$. However, the model of [HUDIS and LIDSKY, 1970] can be used as a starting point. This model is based on the Bohm sheath model [Bонм et al., 1949], in which $T_{\mathrm{i}} \ll T_{\mathrm{e}}$, but modified to include a net ion drift $V_{\mathrm{D}}\left(<C_{\mathrm{s}} \equiv \sqrt{\left(T_{\mathrm{e}}+T_{\mathrm{i}}\right) / m_{\mathrm{i}}}\right)$ at the sheath edge. The ion saturation current in this case can be approximated by

$$
I_{ \pm}=\exp (-1 / 2) n_{\mathrm{i} 0} e A C_{\mathrm{s}} \exp \left(\frac{m_{\mathrm{i}} V_{\mathrm{d}}^{2}}{2 T_{\mathrm{e}}}\right) \exp \left( \pm \frac{\sqrt{m_{\mathrm{i}} T_{\mathrm{i}}} V_{\mathrm{d}}}{T_{\mathrm{e}}}\right)
$$

where $n_{\mathrm{i} 0}$ is the density far from the probe. As mentioned in the previous section, numerical solutions for $I_{\text {sat }}$ from [LAFRAmBoise, 1966] showed that the Bohm sheath model is not strongly dependent on $T_{\mathrm{i}}$ for $T_{\mathrm{i}} \lesssim T_{\mathrm{e}}$. Since $T_{\mathrm{i}} \sim T_{\mathrm{e}}$ in the experiments for which ion flow speeds are measured, Eq. (2.13) can still be used. Substituting $I_{ \pm}$into Eq. (2.12) yields

$$
V_{\mathrm{d}}=\sqrt{\frac{T_{\mathrm{e}}}{T_{\mathrm{i}}}} \sqrt{\frac{T_{\mathrm{e}}}{m_{\mathrm{i}}}} \tanh ^{-1} K
$$

It must be emphasized that, as a general rule, theoretical expressions for electrostatic probes such as Eq. (2.14), and likewise Eq. (2.10), are only correct to within a factor of two. This is not surprising given the non-rigorous justifications 


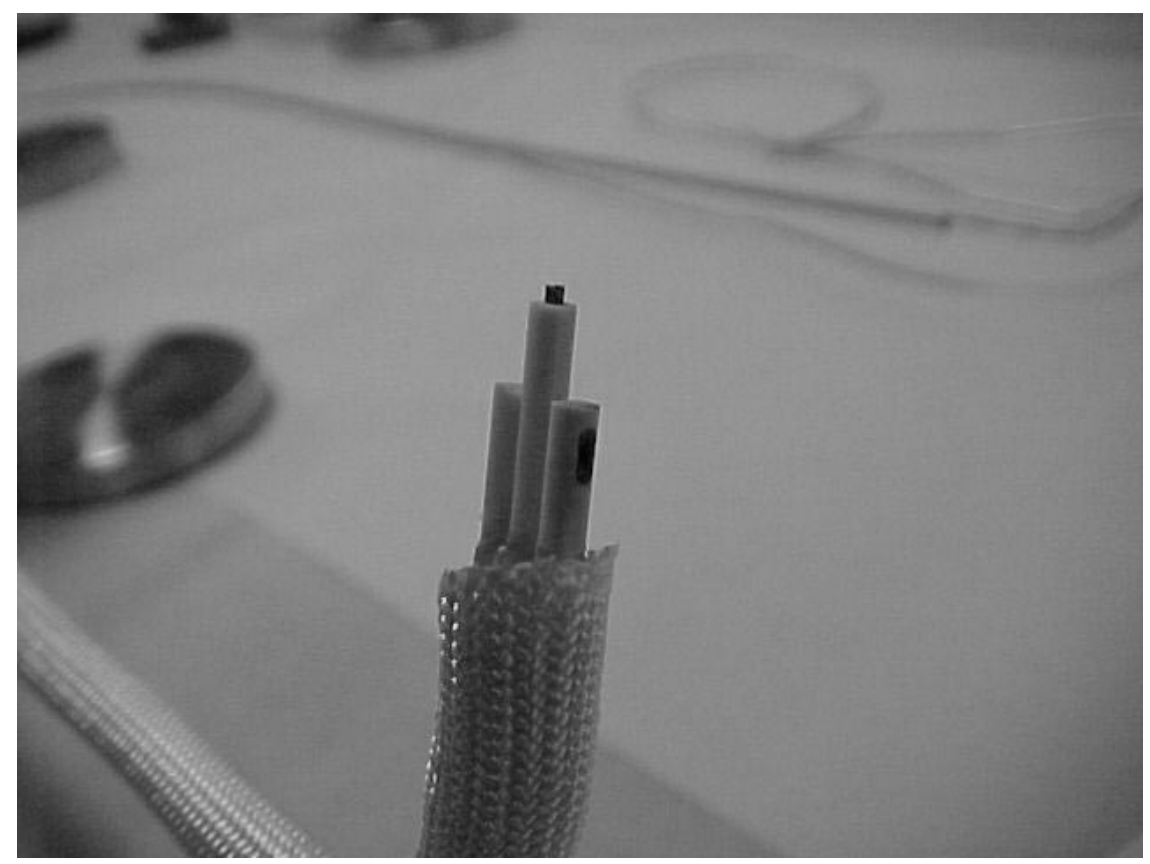

Figure 2.13: Photograph of the Mach probe. Ion saturation current is collected by tungsten tips on either side, which are biased against the floating tip in the center. The width of the probe is $1 / 4$ in.

relied upon in the above discussions. The trends (spatial profile and temporal evolution) which are measured, however, are still meaningful. Ideally, Mach probe results should be checked with an independent measurement. It is found that Mach probe measurements using Eq. (2.14) agree with Doppler shift of ions obtained by spectroscopy to better than $50 \%$. Thus, $V_{\mathrm{D}}$ values reported in this dissertation are based on Eq. (2.14) but include an empirical calibration factor of 0.75 . Details of the independent check are presented in Appendix A.

Physically, the Mach probe, as shown in Fig. 2.13, is housed at the end of a 0.25 in. OD stainless steel shaft, which is covered by fiberglass braid to minimize surface-plasma interactions. The back-to-back electrodes are $1 \mathrm{~mm} \times 3 \mathrm{~mm}$ exposed sections of $1 \mathrm{~mm}$ diameter tungsten rods slid into $2.5 \mathrm{~mm}$ diameter hollow alumina rods. Slots are milled into the sides of the alumina so that each tungsten rod is exposed to the plasma in only one direction. Between these two alumina rods is a third alumina rod with a tungsten rod sticking out of the end by $1 \mathrm{~mm}$. This third electrode gives a reference potential against which the other two electrodes are biased to $-45 \mathrm{~V}$. The tungsten rods are crimped onto copper wire, which are then 


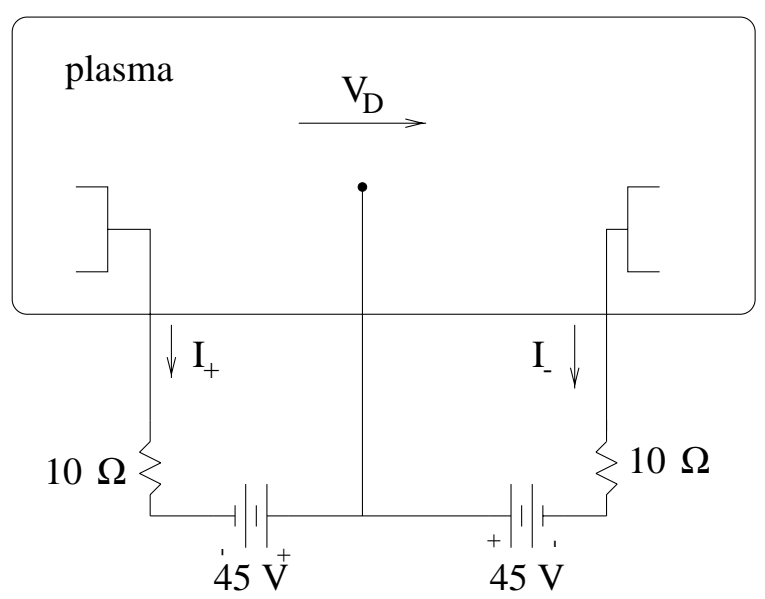

Figure 2.14: Simple schematic of Mach probe circuit, showing collection of ion saturation currents by electrodes facing the flow $\left(I_{+}\right)$and facing away from the flow $\left(I_{-}\right)$.

run down the length of the stainless steel shaft. A $35 \mathrm{ft}$. four-conductor shielded cable brings the signals to the control room. The $45 \mathrm{~V}$ DC bias is implemented with batteries (with parallel $0.1 \mu \mathrm{F}$ and $0.01 \mu \mathrm{F}$ bypass ceramic disc capacitors). Voltages across the $10 \Omega$ resistors are measured using the attenuating optical isolator (10 $\mathrm{M} \Omega$ input impedance), from which the currents $I_{ \pm}=V_{ \pm} /(10 \Omega)$ can be determined. A simple schematic of the bias circuit is shown in Fig. 2.14.

Biasing against a floating electrode rather than machine ground is desirable because the plasma potential in MRX is known to vary by up to $100 \mathrm{~V}$ on very fast time scales of less than $10 \mu \mathrm{s}$. Typical values of current collected are $200 \mathrm{~mA}$.

\subsubsection{Ion Dynamics Spectroscopy Probe (IDSP)}

The IDSP ${ }^{22}$ [FIKSEL et al., 1998] is an insertable optical probe capable of measuring local ion temperature and flow velocities via Doppler spectroscopy. This diagnostic made possible the first rigorous measurements of local $T_{\mathrm{i}}$ in a well-characterized reconnection layer. IDSP Doppler shift measurements also provided an absolute

\footnotetext{
${ }^{22}$ This probe was developed by Dr. Gennady Fiksel at the University of Wisconsin-Madison and generously loaned to the MRX group during September, 1999.
} 
calibration for Mach probe measurements of ion drift speeds, as discussed in Appendix A. This section will describe the probe, the instrumentation used for spectroscopy, and the methods for obtaining a light-intensity versus wavelength spectrum suitable for Doppler broadening analysis.

\section{Description of the IDSP}

A schematic of the IDSP is shown in Fig. 2.15, which indicates its characteristic physical size $(5 \mathrm{~cm})$. The principle of the probe is as follows. Collimated plasma light from two perpendicular lines-of-sight, which are each terminated by viewdumps $7 \mathrm{~cm}$ away, is collected and delivered via fiber optics ${ }^{23}$ to a spectrometer (see below) next to the vacuum vessel. The Doppler broadened spectrum from each sightline yields local $T_{\mathrm{i}}$ information, and the relative Doppler shift between the two sightlines yields ion flow information. Even if only one sightline is used at a time, as was done in this work, Doppler shift could still be obtained by rotating the IDSP $180^{\circ}$ and comparing the relative shift in the data from the two positions.

The probe housing is boron nitride, and the probe shaft is stainless steel. The collimating holes have a diameter of $0.2 \mathrm{~cm}$ and a length of $2 \mathrm{~cm}$. At the end of the holes are fused silica prisms, which protect the fibers from the plasma and can be replaced easily. Figure 2.16 shows the placement of the IDSP in MRX (to scale); the lines-of-sight can be oriented in an $R-Z$ or $R-\theta$ plane, i.e. the IDSP shaft can be rotated. The fiber bundles are brought out of the vacuum vessel (via the probe shaft) and are terminated in standard $11 \mathrm{~mm}$ ferrules. The experiments to be reported used only one ferrule at a time due to incompatibilities in the mounting hardware and due to the limited height of the CCD intensifier.

Due to the relatively large size of the IDSP, it is important to determine whether the probe perturbs the plasma and whether it yields meaningful measurements. A systematic empirical study, described in Appendix B, showed that the global magnetic topology, plasma density, electron temperature, and global ion emission were essentially unaffected by the probe, as long as 10-15 conditioning discharges were fired before each day's experiments. However, the total toroidal plasma current in the layer is reduced by $20 \%$ (typical of shot-to-shot variation) when the IDSP

\footnotetext{
${ }^{23}$ Custom made by Fiberguide Industries, Stirling, NJ. Each bundle consists of 135 thermocoated jacketed fused silica fibers with core diameters of $200 \mu \mathrm{m}$.
} 


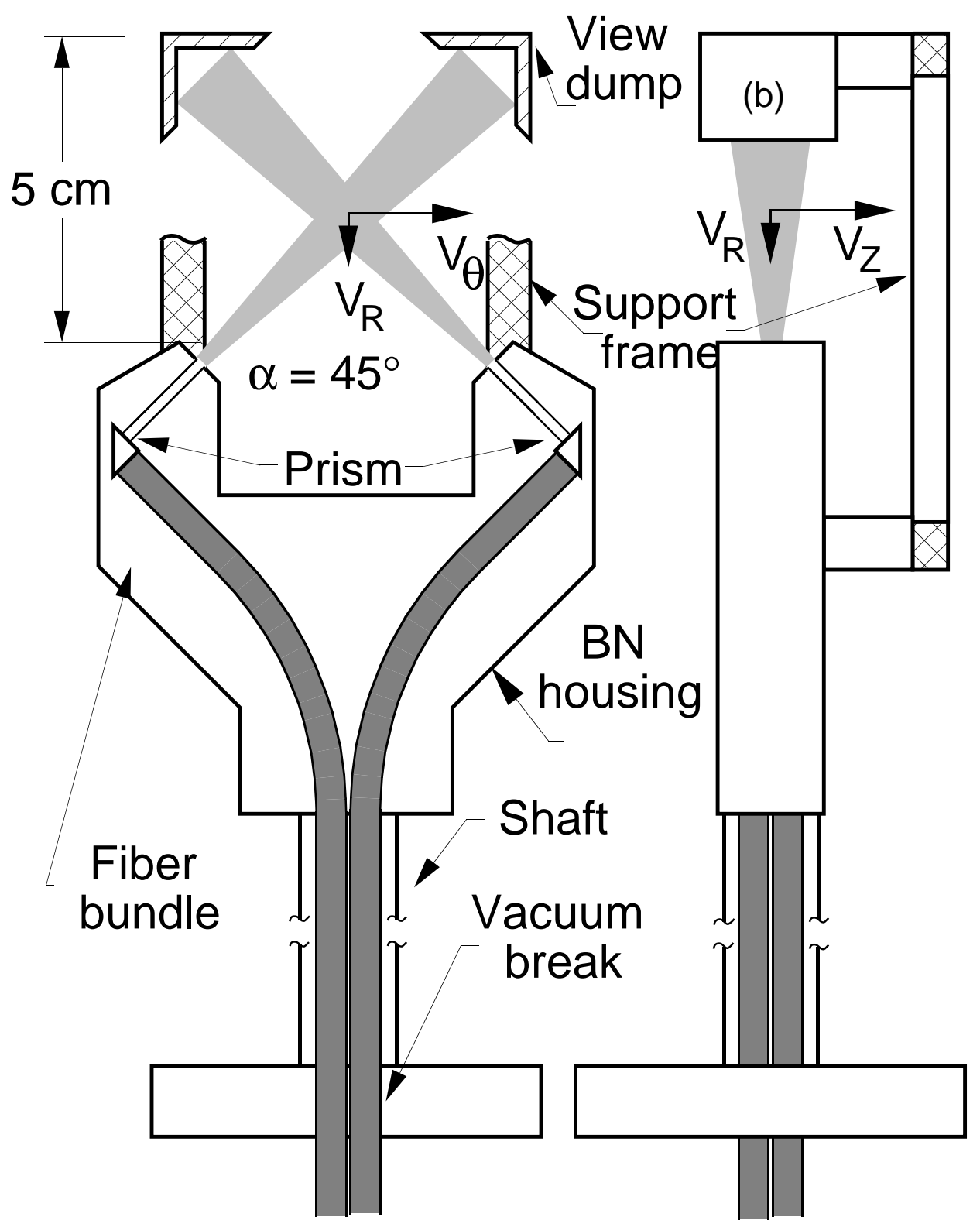

Figure 2.15: Schematic of the IDSP. The boron nitride housing is supported at the end of a stainless steel shaft. Characteristic size, as shown, is $5 \mathrm{~cm}$. The whole probe can be moved in and out through a Wilson seal, and it can also be rotated. This figure taken from [FIKSEL et al., 1998]. 


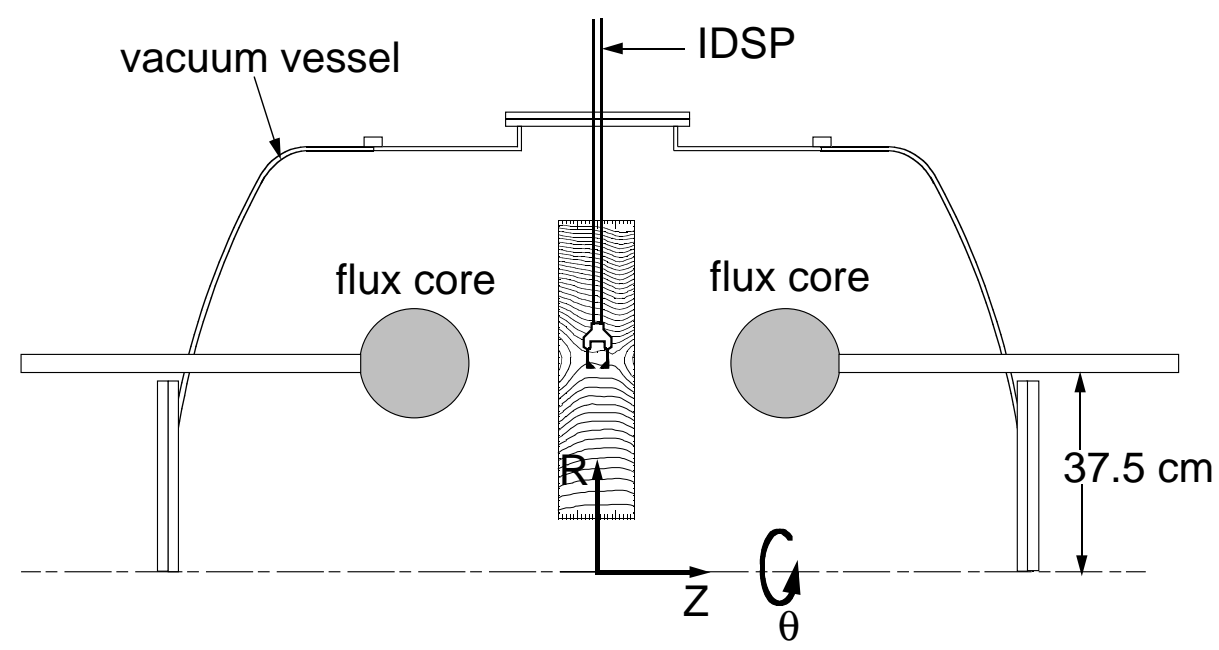

Figure 2.16: Setup of the IDSP in MRX. The probe can be moved in the $R$ direction and rotated. A poloidal flux plot is superimposed onto the schematic to illustrate the position and size of the probe compared to the reconnection region.

is placed at $R=37.5 \mathrm{~cm}$ compared to $R=52.5 \mathrm{~cm}$. Based on this study, it is concluded that the IDSP does not destroy the global reconnection dynamics and that systematic studies of ion heating using this probe are still meaningful, as long as the small effects of the probe are taken into account.

\section{Spectrometer and imaging}

The IDSP fiber optic ferrules are coupled, only one at a time due to hardware incompatibility, to the entrance slit of a $1.0 \mathrm{~m}$ spectrometer ${ }^{24}$ (2400 line/mm grating, $0.05 \AA$ resolution, f/8.7, wavelength range of $185-650 \mathrm{~nm}$ ). The light output from the spectrometer is imaged with an intensified CCD camera ${ }^{25}(512 \times 512$ pixels, 16 bit per pixel), and the images are saved ${ }^{26}$ on a $\mathrm{PC}$ and subsequently transferred to the PPPL UNIX cluster for analysis. The CCD intensifier is triggered and gated using a digital delay/pulse generator. ${ }^{27}$ Gate widths are typically $10-20 \mu \mathrm{s}$ and temporal scans are done on a multi-shot basis by advancing the trigger timing from

\footnotetext{
${ }^{24}$ McPherson model 2061

${ }^{25}$ Princeton Instruments ICCD-MAX

${ }^{26}$ using Princeton Instruments WinSpec 32 frame-grabbing software

${ }^{27}$ Stanford Research Systems DG-535
} 


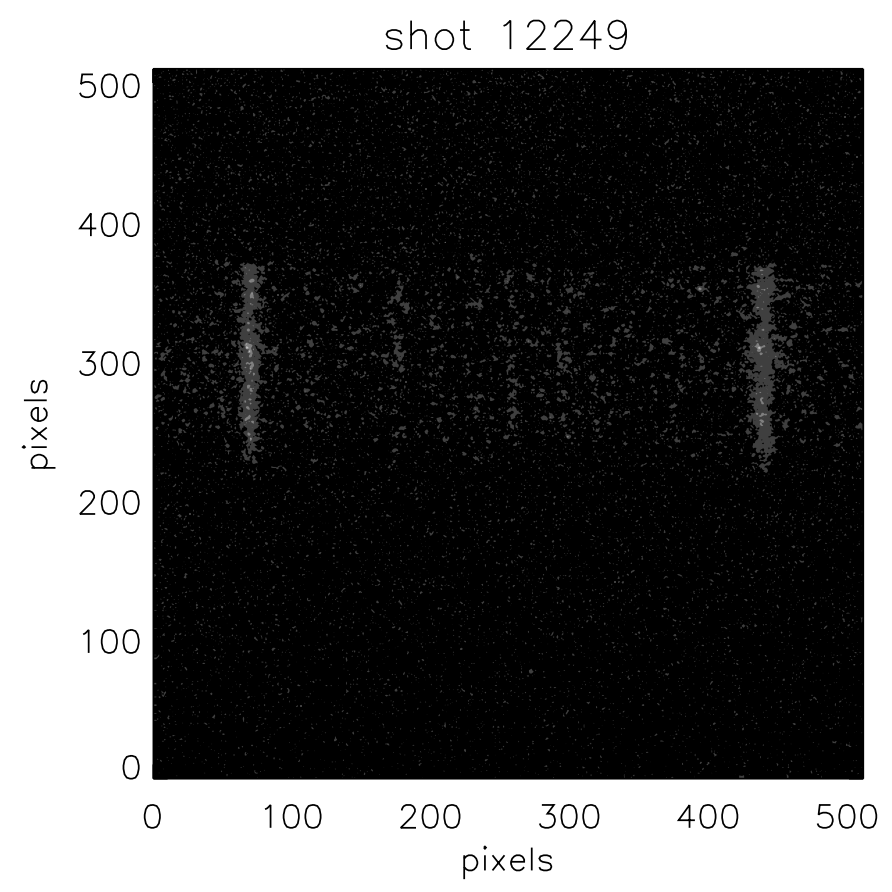

Figure 2.17: CCD image of (left) He I $4713 \AA$ and (right) He II $4686 \AA$ emission lines (CCD gate opening $t=260 \rightarrow 270 \mu \mathrm{s}$ ). The abscissa is proportional to inverse wavelength, and the ordinate corresponds to the vertical position of the spectrometer entrance slit.

shot to shot.

A typical image from a helium discharge is shown in Fig. 2.17. There are two Doppler broadened and shifted emission lines in the figure: He I $4713 \AA$ on the left and He II $4686 \AA$ on the right. The abscissa represents frequency, or equivalently inverse wavelength, of the collected light, and the ordinate is a spatial dimension representing the vertical position along the spectrometer entrance slit at which each individual fiber is placed. This spatial information is not utilized, however, since the image is "binned" in the vertical direction in order to produce sufficient signal level. Thus, in producing an intensity (photoelectrons) versus inverse wavelength (in pixels) plot, as shown in Fig. 2.18, the 2-D data is summed in $y$ at each pixel in $x$. Each photoelectron which strikes the microchannel plate of the intensifier results in 14 CCD "counts" (for the chosen intensifier gain setting of 150) [LEVINTON, 1999]. Therefore, the number of counts recorded should be divided by 14 for the number of photoelectrons collected. Invoking Poisson statistics, this means that the detection 


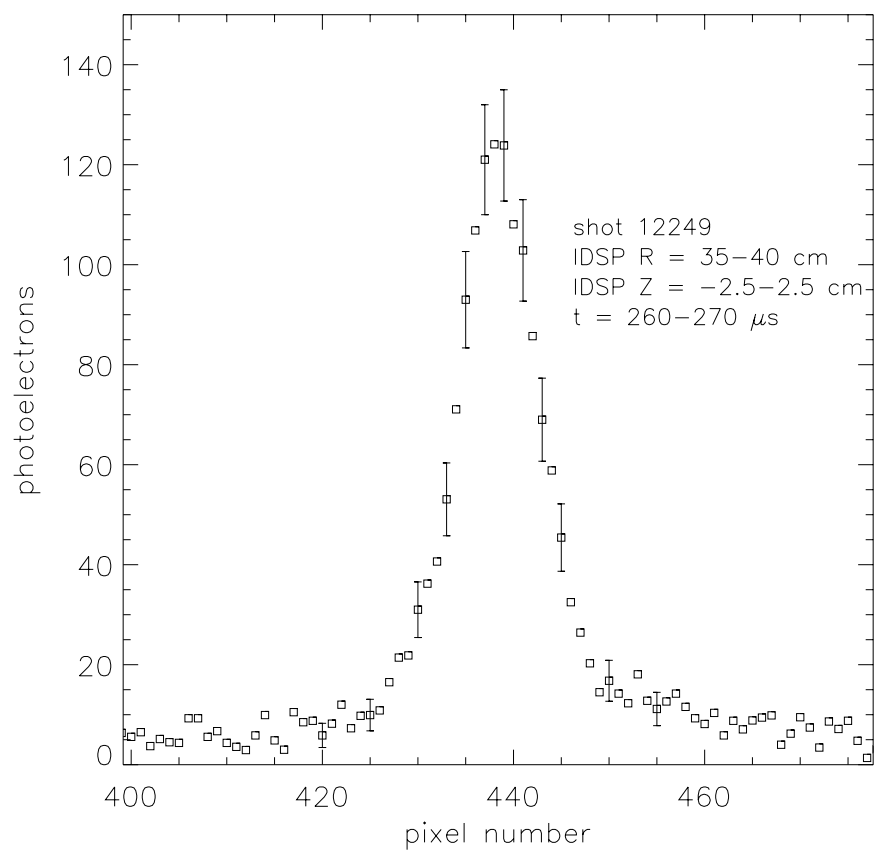

Figure 2.18: Photoelectrons versus CCD pixel number (representing inverse wavelength) after adding the light in the vertical direction of the spectrometer slit. Error bars represent the photoelectron detection error using Poisson statistics. 


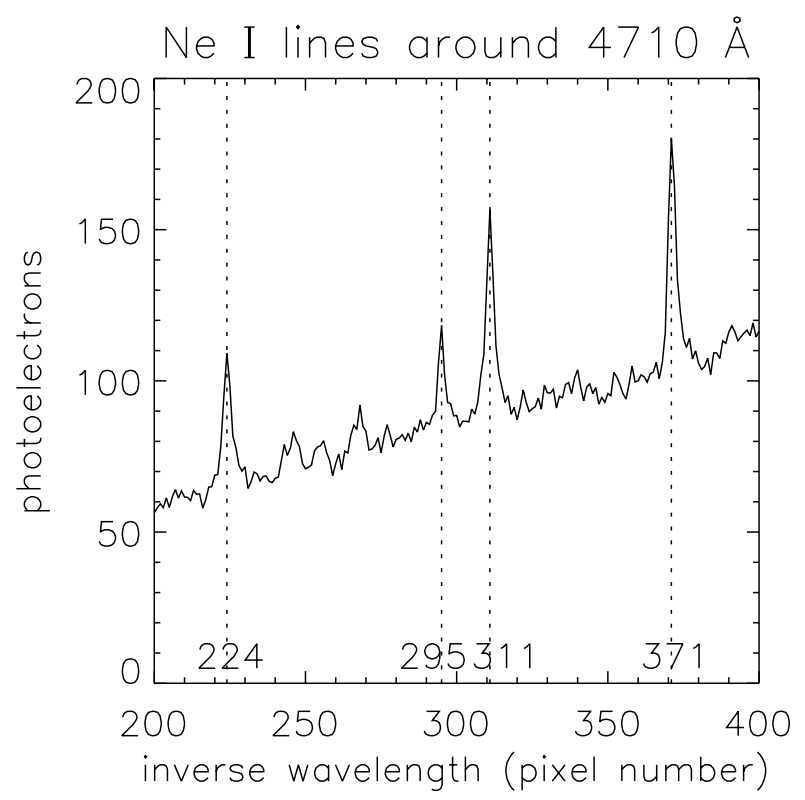

Figure 2.19: Ne I emission lines from a discharge lamp: $4715.35 \AA, 4710.07 \AA$, $4708.86 \AA$, and $4704.40 \AA$ (from left to right). Corresponding pixel numbers are shown on the plot. CCD pixel resolution is calculated to be $0.074 \AA /$ pixel.

error, shown as error bars in Fig. 2.18, is simply the square root of the number of photoelectrons at each pixel. ${ }^{28}$

In order to determine the full-width half maximum (FWHM) or Doppler shift of the measured spectrum in $\AA$, a wavelength per pixel calibration is needed. This is obtained using a neon discharge lamp. Figure 2.19 shows a plot of four Ne I lines with known central wavelengths: $4715.35 \AA, 4710.07 \AA, 4708.86 \AA$, and $4704.40 \AA$ (from left to right). It can thus be determined that the CCD pixel resolution is $0.074 \AA$ per pixel. Obviously, an absolute calibration between wavelength and pixel number can also be obtained from Fig. 2.19. However, this procedure was not repeated for the spectrometer setting used for real helium experiments because an absolute calibration was not necessary for $T_{\mathrm{i}}$ and $V_{\mathrm{i}}$ measurements using the IDSP. (Thus abscissa values in Figs. 2.17 and 2.18 are in pixel number rather than

\footnotetext{
${ }^{28}$ There is an issue of pixel-to-pixel correlation in the CCD which could increase the calibrated number from 14 to 50 [TRINTCHOUK, 2000]. However, the only effect this could have is to increase the line profile fitting error of $T_{\mathrm{i}}$ from $<10 \%$ to approximately $15 \%$, still smaller than the dominant source of uncertainty which is shot-to-shot variation.
} 
wavelength.)

\subsubsection{Data acquisition system}

The data acquisition system consists of a desktop computer running National Instruments LabVIEW ${ }^{29}$ and five CAMAC crates containing more than 150 digitizer channels of varying sampling times, dynamic range, and bit resolution (summarized in Table 2.2). The CAMAC crates are each controlled by a LeCroy 8901A GPIB interface. All the magnetic probe signals are first integrated electronically and then digitized using LeCroy 2264 digitizers. Triple Langmuir probe and Mach probe signals are digitized using LeCroy 8210 digitizers. High frequency digitizers (LeCroy 8828B and Transiac T2001) are available for studying fluctuations or other quantities requiring better than $2 \mu$ s time resolution. The digitized data are analyzed on the PPPL UNIX cluster using IDL. ${ }^{30}$

\begin{tabular}{||c|c|c|c|c||}
\hline Digitizer & 2264 & 8210 & TR8828B & T2001 \\
\hline \hline Channels & 8 & 4 & 1 & 1 \\
\hline Sampling time used & $2 \mu \mathrm{s}$ & $2 \mu \mathrm{s}$ & $5 \mathrm{~ns}$ & $10 \mathrm{~ns}$ \\
\hline Bits & 8 & 10 & 8 & 8 \\
\hline Dynamic range & $\pm 256 \mathrm{mV}$ & $\pm 5 \mathrm{~V}$ & $\pm 256 \mathrm{mV}, \pm 2.56 \mathrm{~V}$ & $\pm 256 \mathrm{mV}$ \\
\hline Resolution (mV/bit) & 2 & 10 & 2,20 & 2 \\
\hline Input impedance & $50 \Omega$ & $1 \mathrm{M} \Omega$ & $50 \Omega$ & $50 \Omega$ \\
\hline
\end{tabular}

Table 2.2: Specifications of the various digitizers used for data acquisition.

\footnotetext{
${ }^{29}$ Prior to July, 1999: Apple Power Macintosh 8100/100AV running LabVIEW 3.1; after July, 1999: Sun Sparc 10 (UNIX cluster: lupus.pppl.gov) running LabVIEW 4.1.

${ }^{30}$ Interactive Data Language from Research Systems, Inc., Boulder, CO; custom IDL routines are located on the PPPL UNIX cluster at /mrxdata/idl/ and were developed over the past four years by members of the MRX group.
} 


\section{Chapter 3}

\section{Characterizing the Reconnection Process}

HOROUGH characterization of the reconnection process is necessary for
demonstrating cause and effect between reconnection and any ion heating.
It is also necessary for gaining insight into the particular heating mechanisms. A substantial part of this dissertation research was devoted to characterizing in detail the physics of the reconnection process in MRX.

In every plasma discharge, more than 135 independent magnetic probe signals as well as triple Langmuir probe measurements of electron density and temperature in the reconnection region are obtained, the experimental details of which are discussed in Ch. 2. This chapter is concerned with analyzing the magnetic probe and triple Langmuir probe data sets, calculating key physics quantities from the data sets, and developing both a qualitative and quantitative understanding of the reconnection process in MRX.

The chapter is organized as follows. The global magnetic field topology is constructed from the individual probe measurements and visualized using computer graphics. Key signatures of reconnection are then easily identified by visualizing the time evolution of the poloidal magnetic field vectors and contours of the poloidal magnetic flux function. Next, high resolution 1-D plots of the reconnecting field are shown, revealing sharp gradients. From these high-resolution measurements, the current density profile and the thickness of the reconnection layer can be calculated by fitting an analytic function to the reconnecting field profile. Basic quantities such as the Alfvén speed, the classical resistivity, and the ion skin depth can be 
calculated using the magnetic probe and triple Langmuir probe data. Further derived quantities such as the reconnection rate, the reconnection electric field, the plasma resistivity, and the dissipated magnetic energy will then be presented. The chapter concludes with a summary of MRX reconnection physics results.

\subsection{Magnetic field topology}

The clearest way of identifying reconnection is to observe a change in magnetic field topology and the annihilation of magnetic flux. Experimentally, this is achieved by visualizing the experimentally measured magnetic field data in 2-D plots.

As discussed in Sec. 2.3.1, the field reversal region is monitored by the 90channel probe array, which yields all three components of $\mathbf{B}$ at 30 spatial positions. The data set is interpolated down to a grid size of $1 \mathrm{~cm}$ using the IDL library routine min_curve_surf. The interpolated $B_{\mathrm{Z}}(Z, R, t)$ and $B_{\mathrm{R}}(Z, R, t)$, which together comprise the poloidal magnetic field $\mathbf{B}_{\mathbf{P}}$, are then illustrated in a $2-\mathrm{D}$ vector plot using the IDL library routine velovect. The 2 -D toroidal field $B_{\theta}$ profile can be visualized easily as a contour plot or 3-D surface plot.

In Fig. 3.1, two vector plots are shown for null-helicity reconnection, representing two different situations in the experiment. The first is early in the discharge sequence before a strong reconnection electric field is established. In this case, the merging field lines form an X-point with a $90^{\circ}$ separatrix, indicative of vacuum reconnection in which $\left(\boldsymbol{\nabla} \times \mathbf{B}_{\mathbf{P}}\right)_{\theta}=\partial B_{\mathrm{R}} / \partial Z-\partial B_{\mathrm{Z}} / \partial R=\mu_{0} j_{\theta}=0$. The second is during the pull reconnection phase during which a field null region elongated in the $Z$ direction develops, a key indicator of reconnection due to the existence of a large $\left(\boldsymbol{\nabla} \times \mathbf{B}_{\mathbf{P}}\right)_{\theta} \approx-\partial B_{\mathbf{Z}} / \partial R$ and therefore a large toroidal sheet current. From the vector plot, the approximate half-length of the current sheet, $L \approx 10 \mathrm{~cm}$, can be estimated. It should be emphasized that the vector plots shown in Fig. 3.1 represent the area covered by the 90-channel probe and are taken in one plasma discharge.

Magnetic flux is an especially useful quantity in analyzing plasmas which satisfy the ideal MHD approximation $\left(S \gg 1, \rho_{\mathrm{i}} \ll L, V_{\mathrm{A}} \ll c\right)$ because motion of magnetic field lines can be envisioned to be "frozen-in" to the motion of the plasma and vice versa. Therefore, calculating and displaying the poloidal magnetic flux function $\psi$ 


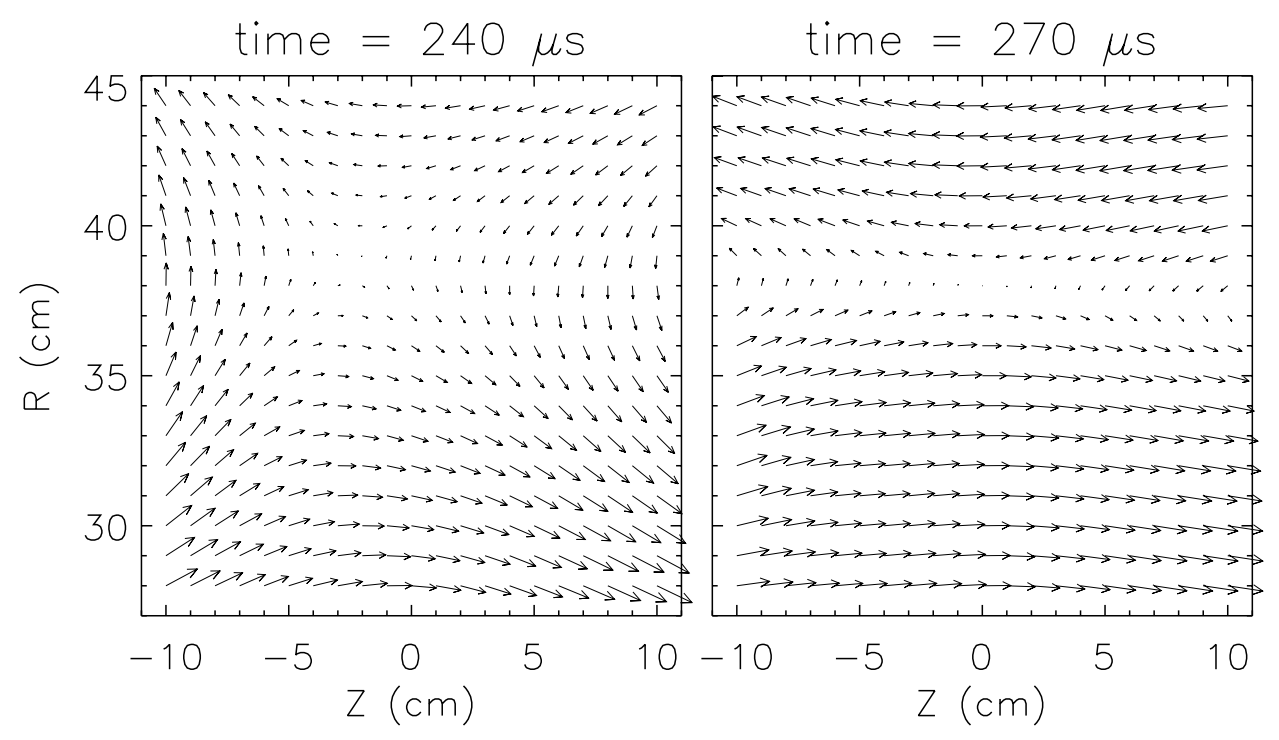

Figure 3.1: Vector plots of the poloidal magnetic field (left) early during the discharge when the field is vacuum-like and (right) later during the discharge when pull reconnection is induced and a long narrow layer is formed (shot 3453, null-helicity).

can reveal much information about both the magnetic field topology and the global plasma motion.

Experimentally, the poloidal flux function $\psi$ is determined as follows,

$$
\psi(Z, R, t)=\int_{0}^{R} 2 \pi R^{\prime} B_{\mathrm{Z}}\left(Z, R^{\prime}, t\right) \mathrm{d} R^{\prime},
$$

where axisymmetry $\partial / \partial \theta=0$ is invoked and $\psi$ is defined to be zero at the machine axis $(R=0 \mathrm{~cm})$. Contour plots of $\psi$ early in the discharge and during pull reconnection are shown in Fig. 3.2. Magnetic field data from $R=6 \rightarrow 24 \mathrm{~cm}$ is taken with the 60 -channel probe, and data from $R=28 \rightarrow 44 \mathrm{~cm}$ is taken with the 90-channel probe simultaneously. The field is uniform near the axis, and therefore the value at $R=6 \mathrm{~cm}$ is used for $R<6 \mathrm{~cm}$. Because $B_{\mathrm{Z}}$ data are known only at the toroidal positions of the two magnetic probe arrays (which are situated $90^{\circ}$ away from each other in $\theta$ ), the calculation of $\psi$ relies upon the assumption of toroidal symmetry. Since MRX is a toroidal device with good toroidal symmetry, it is expected that the plasma should exhibit good global toroidal symmetry. Several experimental observations confirm this expectation. First, rotating the 1-D probe 

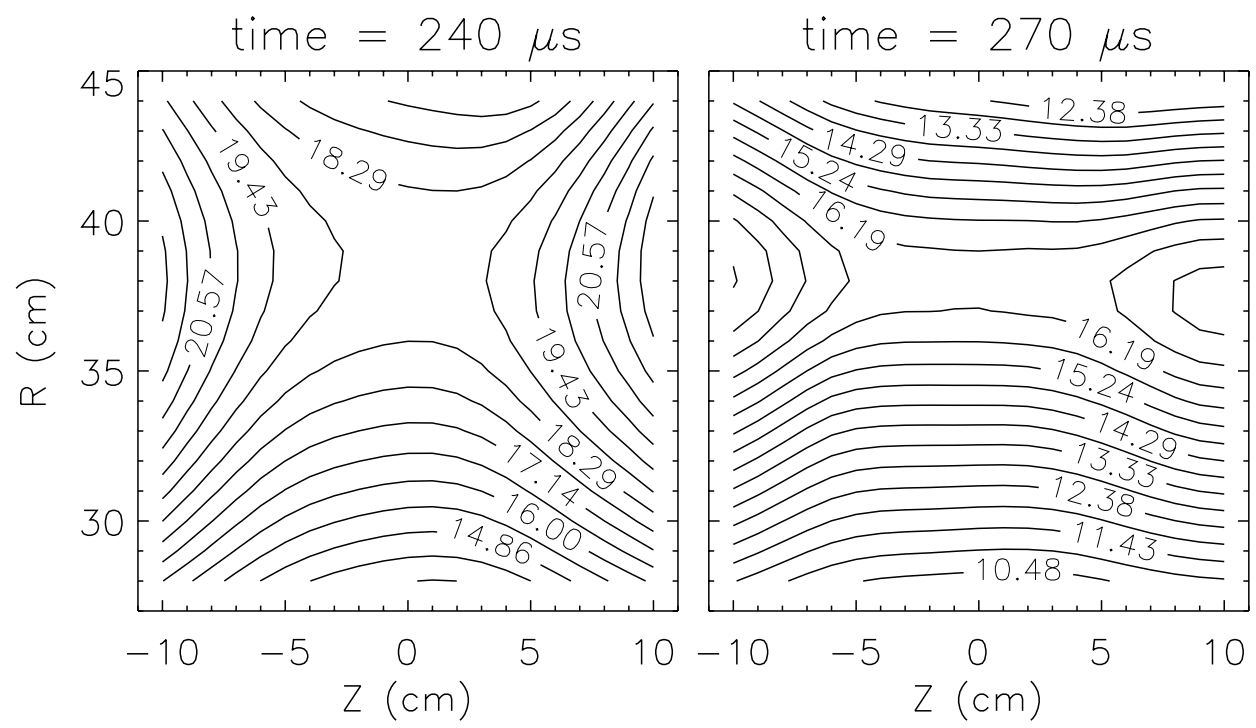

Figure 3.2: Contour plots of the poloidal magnetic flux function $\psi$ (contour labels in units of $\mathrm{mWb}$ ): (left) early during the discharge when the field is vacuum-like and (right) later during the discharge when pull reconnection is induced and a toroidal current sheet is formed (shot 3453).

to a few different $\theta$ positions shows $B_{\mathrm{Z}}(R)$ profiles with similar magnitude at the edge of the layer and similar null point $R$ position (within a few percent). Second, there is very good agreement between the vector plots (which do not invoke axisymmetry) in Fig. 3.1 and the contour plots of $\psi$ (which do) in Fig. 3.2. Third, radial scans of the 90-channel probe (within the same toroidal plane) over multiple discharges yield virtually the same $\psi$ contour plot, as shown in Sec. 3.7.1.1

Calculations of $\psi$ are performed numerically using the IDL library routine int_tabulated, which is based on a closed five-point Newton-Cotes formula. ${ }^{2}$ It must be emphasized that the $B_{\mathrm{Z}}$ data used here are taken every $4 \mathrm{~cm}$ in both the $R$ and $Z$ directions and that the smoothness of the $\psi$ plots on scales smaller than $4 \mathrm{~cm}$ is a result of interpolating $B_{\mathrm{Z}}(Z, R, t)$ spatially. The flux plots clearly reveal the topology of the magnetic field, which is a vacuum-like X-point early in the discharge and an elongated "double-Y" shaped region during pull reconnection, which is similar to the idealized picture of the Sweet-Parker model. The narrow

\footnotetext{
${ }^{1}$ Therefore, unless otherwise noted, global toroidal symmetry, i.e. $\partial / \partial \theta=0$, will be assumed in all subsequent sections of the dissertation.

${ }^{2}$ For details on the numerical scheme, see e.g. [BURDEN and FAIRES, 1993].
} 
reconnection layer persists for approximately 20-30 $\mu \mathrm{s}$, or roughly 15-20 Alfvén transit times.

\subsection{Thickness of the layer and current density profile}

The thickness of the reconnection layer $\delta$ is an important parameter because it is closely related to the physical processes which determine the reconnection rate, such as resistive dissipation of the current sheet as well as the mass flow into and out of the reconnection region. In the case of pull reconnection, the $B_{\mathrm{Z}}$ component undergoes reconnection and large gradients are expected in $R$. Local features with spatial scales smaller than the $4 \mathrm{~cm}$ resolution of the 90 channel magnetic probe array are studied with the 29 channel 1-D magnetic probe array, which has $0.5 \mathrm{~cm}$ resolution in the reconnection region. The 1-D magnetic probe array is usually placed at the midplane $(Z=0 \mathrm{~cm})$, giving a high resolution radial profile of the reconnecting field $B_{\mathrm{Z}}(R)$ across the reconnection layer. The time evolution of $B_{\mathrm{Z}}(R)$ during pull reconnection is shown in Fig. 3.3. Note that the field-reversal region is spanned by more than ten data points, ensuring that the profile measurement is not limited by probe resolution.

It was noted that the $B_{\mathrm{Z}}$ measurements could be fit very well to several analytic functions: arctangent, hyperbolic tangent, and error function, for which the corresponding $j_{\theta}$ profile would be Lorentzian, hyperbolic secant squared, and Gaussian, respectively. The fitting is a convenient and precise way to determine the peak current density and sheet thickness $\delta$. The different functions give virtually indistinguishable fitted $B_{\mathrm{Z}}$ profiles, although the error function always has a slightly larger reduced- $\chi^{2} \cdot{ }^{3}$ It was then noticed that the hyperbolic tangent profile corresponds to the well-known and oft-quoted collisionless current sheet model of [HARRIS, 1962], despite significant physical differences between the MRX current sheet and the assumptions of the model. This problem is addressed separately in Sec. 3.7.3.

The close agreement between the data and Harris' solution led to the routine

\footnotetext{
${ }^{3}$ See e.g. [Bevington and Robinson, 1992].
} 


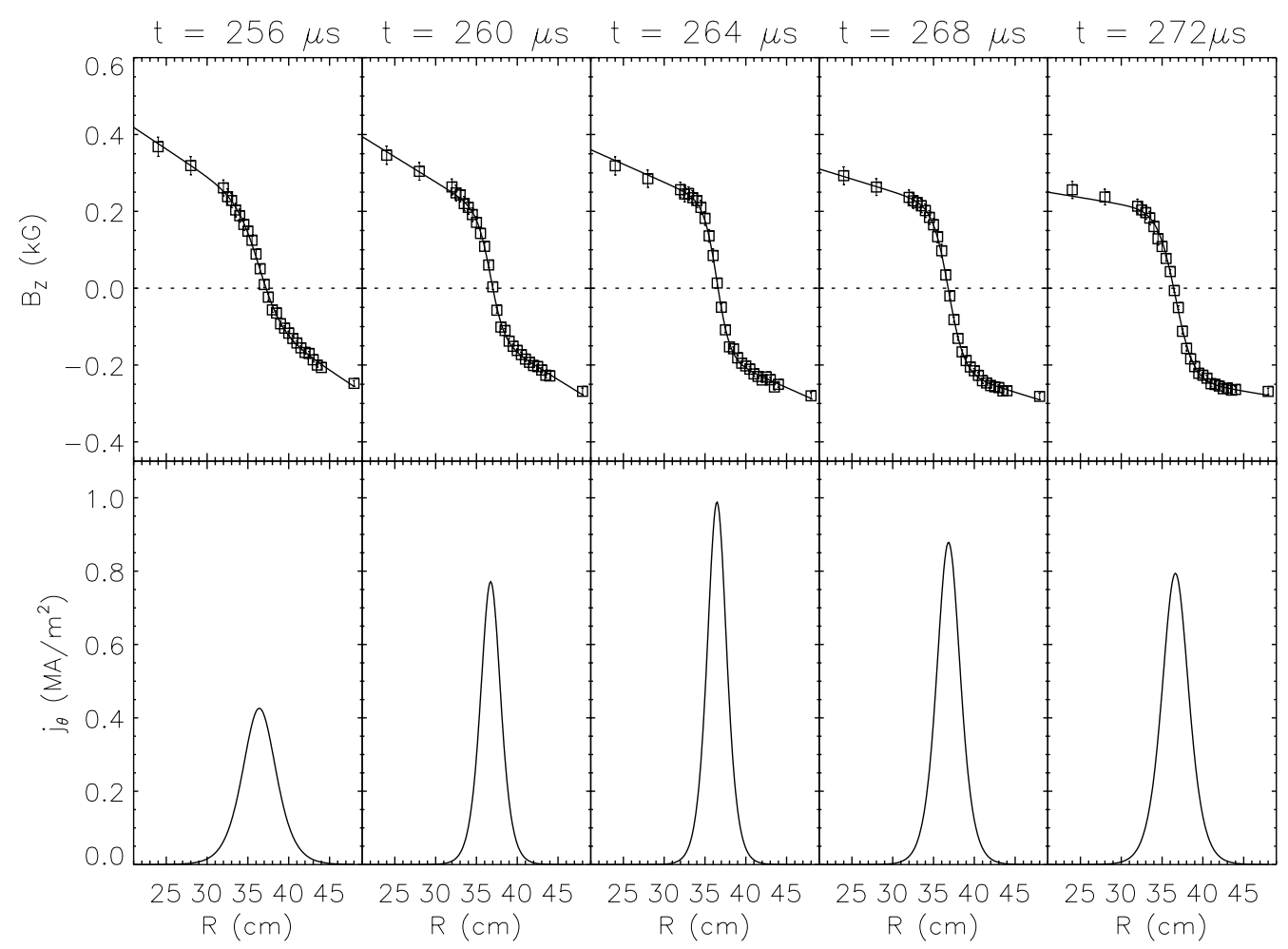

Figure 3.3: Time evolution of (top) reconnecting field $B_{\mathrm{Z}}$ profile and (bottom) current density $j_{\theta}$ taken from a single plasma discharge. Measured data points are represented by squares, and solid lines are from the fitting functions. Error bars for $B_{\mathrm{Z}}$ represent the measurement error of the magnetic probe system. This figure is to be published in [YAMADA et al., 2000].

use of the hyperbolic tangent as a fitting function for $B_{\mathrm{Z}}(R)$,

$$
B_{\mathrm{Z}}(R)=a_{0}+a_{1} R+a_{2} \tanh \left(\frac{R-R_{0}}{\delta}\right),
$$

where $a_{0}$ and $a_{1}$ are determined from the EF and initial quadrupole fields, is fit to the $B_{\mathrm{Z}}(R)$ data using the IDL library routine curvefit. The field at the "knee" determines $a_{2}$ and is also used to define the upstream Alfvén speed $V_{\mathrm{A}}$. For elongated layers in the $Z$ direction, the toroidal current density $j_{\theta}$ can be deduced from 
Ampere's law,

$$
j_{\theta}(R)=\frac{1}{\mu_{0}}\left(\frac{\partial B_{\mathrm{R}}}{\partial Z}-\frac{\partial B_{\mathrm{Z}}}{\partial R}\right) \approx-\frac{1}{\mu_{0}} \frac{\partial B_{\mathrm{Z}}}{\partial R},
$$

yielding

$$
j_{\theta}(R)=j_{0} \operatorname{sech}^{2}\left(\frac{\mathrm{R}-\mathrm{R}_{0}}{\delta}\right) .
$$

The factor $a_{1}$ does not appear in Eq. (3.4) because it is cancelled exactly by $\partial B_{\mathrm{R}} / \partial Z$ of the background quadrupole field. The profile of $j_{\theta}(R)$ is illustrated in the bottom row of Fig. 3.3, from which the peak current density can also be determined. From Eqs. (3.2) and (3.4), $\delta$ is determined precisely. It should be mentioned that although the three different fit functions give virtually indistinguishable $B_{\mathrm{Z}}$ profiles, the derived values of peak $j_{\theta}$ and $\delta$ can be different by as much as $30 \%$. This uncertainty may be considered an upper bound in the measurement uncertainty of $\delta$ and $j_{0}$, which are used in calculating other quantities and interpreting reconnection models, to be discussed in the following sections.

\subsection{Electron density and temperature profiles}

Electron density $n_{\mathrm{e}}$ and temperature $T_{\mathrm{e}}$ are measured by a triple Langmuir probe which is scanned spatially to yield profiles in $R$ and $Z$. In general, both $n_{\mathrm{e}}$ and $T_{\mathrm{e}}$ rise rapidly early in the formation sequence and then stay roughly constant during the reconnection phase. Spatially, both $n_{\mathrm{e}}$ and $T_{\mathrm{e}}$ are peaked radially near the reconnection layer, as shown in the third and fourth rows of Fig. (3.4), but are roughly uniform along the $Z$ direction. The radial profiles of $n_{\mathrm{e}}$ and $T_{\mathrm{e}}$ are generally asymmetric about the peak of the current sheet, higher for $R>40 \mathrm{~cm}$ than for $R<35 \mathrm{~cm}$. This feature is consistent with the existence of a stationary pressure balance, $p+B^{2} / 2 \mu_{0}=$ constant, since $B^{2}$ is slightly smaller on the outside compared to the inside. The establishment of pressure balance is discussed further in Sec. 3.7.3

Using the measured $B_{\mathrm{Z}}, n_{\mathrm{e}}$, and $T_{\mathrm{e}}$ in Fig. (3.4) (shown for null-helicity reconnection), other critical quantities can be calculated, such as the Alfvén speed 

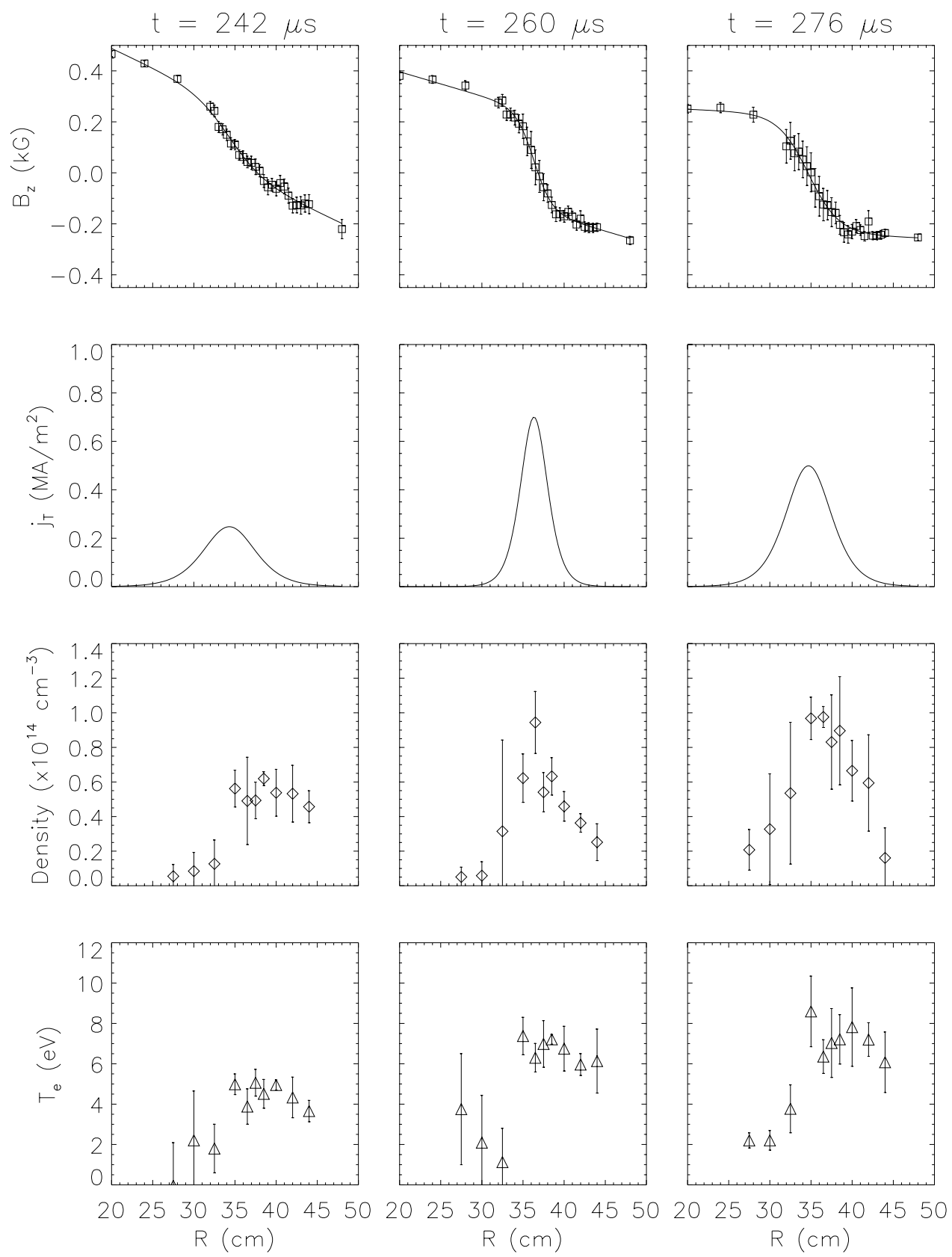

Figure 3.4: Radial profiles of (from top to bottom) $B_{\mathrm{Z}}, j_{\theta}$ (or $j_{\mathrm{T}}$ for toroidal), $n_{\mathrm{e}}$, and $T_{\mathrm{e}}$ in null-helicity hydrogen discharges. The solid line in the $B_{\mathrm{Z}}$ graph is the fitted tanh function, and the $j_{\mathrm{T}}$ ( or $j_{\theta}$ ) plot is the derivative of tanh, or sech $^{2}$. Error bars represent one standard deviation of the statistical spread among multiple plasma discharges. 
$V_{\mathrm{A}} \approx 6.5 \times 10^{6} \mathrm{~cm} / \mathrm{s}$ (using $B=300$ Gauss and $n_{\mathrm{e}}=10^{14} \mathrm{~cm}^{-3}$ ), the classical Spitzer resistivity $^{4}$ [SPITZER, 1962] $\eta_{\perp} \approx 5 \times 10^{-5} \Omega \mathrm{m}=5.6 \times 10^{-15} \mathrm{~s}$ in Gaussian units (using $T_{\mathrm{e}}=8 \mathrm{eV}$ ), and the Lundquist number $S \equiv \mu_{0} L V_{\mathrm{A}} / \eta_{\perp} \approx 163$ (using $L=10 \mathrm{~cm}$ ). Also, the ion skin depth $c / \omega_{\text {pi }}$, which has been shown in two-fluid theory and simulations [SHAY et al., 1998] to be the relevant length scale for the thickness of the reconnection layer, is determined to be approximately $2.3 \mathrm{~cm}$.

In general, electron temperature is consistently higher by up to a factor of two in helium discharges $\left(T_{\mathrm{e}} \approx 15-20 \mathrm{eV}\right)$ compared to hydrogen discharges $\left(T_{\mathrm{e}} \approx 8-\right.$ $10 \mathrm{eV})$. The discrepancy exists for the same discharge voltage. This difference is not understood at present but could be related to one or more of the following (all speculations): (1) different ionization process due to higher ionization potential of $\mathrm{He}$ and necessity of breaking diatomic $\mathrm{H}_{2}$, (2) lower plasma density in He discharges, which translates to higher temperature for given pressure, (3) faster energy exchange in $\mathrm{H}$, and (4) different proportion of heating to electrons versus ions due to reconnection depending on ion mass. The difference in $T_{\mathrm{e}}$ between $\mathrm{H}$ and $\mathrm{He}$ discharges contributes to important plasma parameter differences, as shown in Table 2.1. The differences between hydrogen and helium discharges warrant detailed further investigation.

\subsection{Reconnection rate}

The reconnection rate is traditionally defined as a normalized quantity $V_{\text {in }} / V_{\mathrm{A}}$, where $V_{\text {in }}$ is the inflow speed of both the plasma and the "frozen-in" magnetic flux toward the reconnection layer. The Alfvén speed is known from the magnetic field and density measurements, as discussed in the prior sections, and $V_{\text {in }}$ can be deduced by invoking the flux-freezing condition, ${ }^{5}$

$$
\frac{\mathrm{d} \psi}{\mathrm{d} t}=0 \Rightarrow \mathbf{V} \cdot \boldsymbol{\nabla} \psi=-\frac{\partial \psi}{\partial t}
$$

\footnotetext{
${ }^{4}$ The relevant resistivity, perpendicular or parallel, depends on whether reconnection is cohelicity or null-helicity. This is discussed in 3.5.

${ }^{5}$ See e.g. [FREIDBERG, 1987].
} 


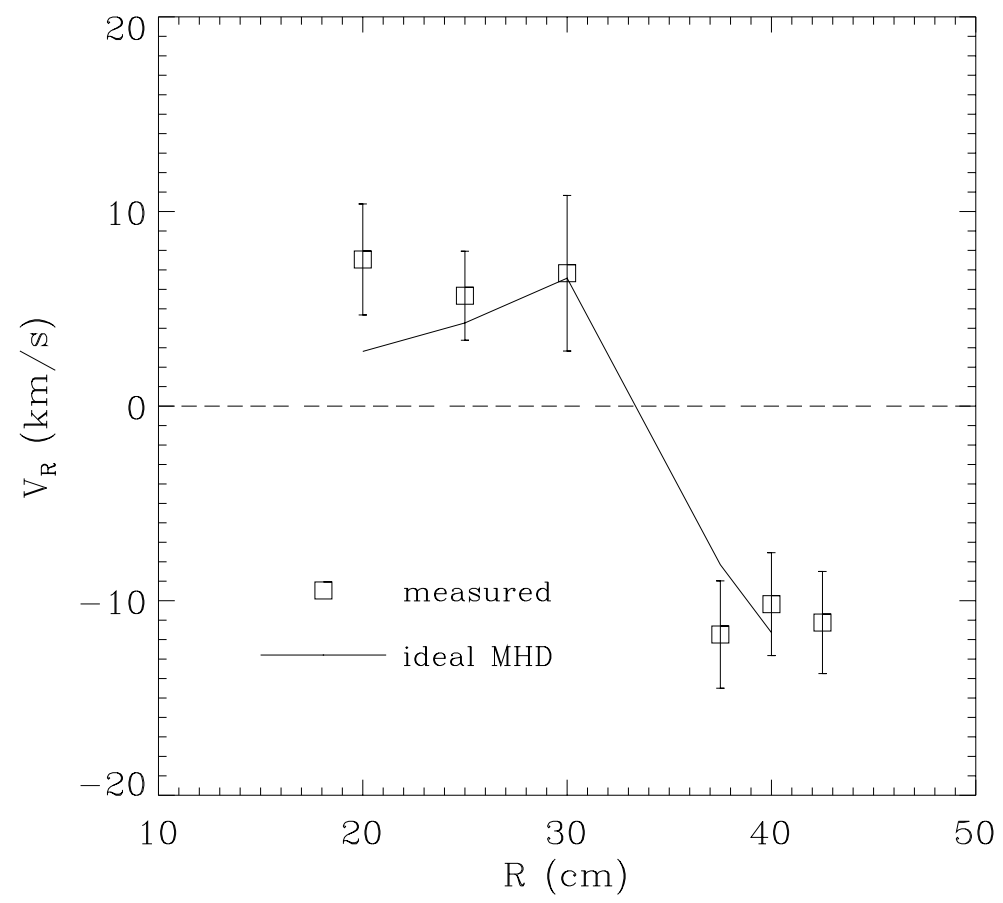

Figure 3.5: Radial profiles of reconnection speed $V_{\mathrm{R}}$ at $Z=0 \mathrm{~cm}$ determined from motion of frozen-in magnetic flux (solid line) and from Mach probe measurements (square data points) averaged over the duration of pull reconnection $(t=260$ $280 \mu \mathrm{s})$.

In regions where the MHD criteria are satisfied, $V_{\text {in }}\left(=V_{\mathrm{R}}\right.$ for pull reconnection) can be deduced from Eq. (3.5) using the experimental values for $\psi(Z, R, t)$,

$$
V_{\mathrm{R}}(Z, R, t)=-\left.\left(\frac{\partial \psi}{\partial t}\right)\left(\frac{\partial \psi}{\partial R}\right)^{-1}\right|_{(Z, R, t)},
$$

where axisymmetry and $\partial \psi / \partial Z \approx 0$ are used; the latter can be verified easily from the $\psi$ data. This is essentially the radial $E \times B$ drift speed. The derivatives are performed using the IDL library routine deriv. The radial profile of the resulting $V_{\mathrm{R}}(R)$ at the midplane $(Z=0 \mathrm{~cm})$ is shown in Fig. 3.5 (solid line), showing that the fluid plasma and magnetic flux are being brought toward the reconnection region at speeds on the order of $0.1 V_{\mathrm{A}}$. The calculation of $V_{\mathrm{R}}$ diverges at the layer since $\partial \psi / \partial R \rightarrow 0$ there; in the figure the divergent points are not shown and instead replaced by interpolated data. This result is independently verified by 
direct measurements of $V_{\mathrm{R}}$ using a Mach probe, also shown in Fig. 3.5. ${ }^{6}$

The scaling of reconnection rate with $S$ can be studied in detail, and one of the primary MRX results to-date is the verification of a generalized Sweet-Parker model [Ji et al., 1998; Ji et al., 1999] which includes the effects of non-classical resistivity, downstream pressure, and finite plasma compressibility. This result is discussed in more detail in Sec. 3.7.2

\subsection{Plasma resistivity}

The physical effects that dominate in determining the plasma resistivity are really at the heart of the reconnection problem, which can be thought of in the following simple manner. Destruction of magnetic flux (e.g. by the forcing together of two coronal flux tubes by footpoint motions in the photosphere) induces a selfconsistent electric field and current sheet in the plasma. However, the current is limited because the current-carrying particles can be scattered by collisions or wave fields, or they can leave the reconnection region due to inertial effects. These processes dissipate the current and can heat the plasma, in effect converting field energy to plasma kinetic energy. In MRX, the effective plasma resistivity $\eta^{*}$ has been observed to be as large as ten times the classical Spitzer value $\eta_{\mathrm{Sp}}$ in the more collisionless regimes of null-helicity discharges, as shown in Fig. 3.6. Collisionality is characterized by the electron Coulomb mean free path $\lambda_{\text {mfp }}$ divided by the current sheet thickness $\delta$. The measured resistivity $\eta^{*}$ and the classical value $\eta_{\text {sp }}$ are shown as a function of density in Fig. 3.7, indicating that lower densities correlate with higher $\eta^{*}$. Collisions with neutrals are estimated to be unimportant, and a more detailed assessment of this effect is the subject of current MRX research. As mentioned in the previous section, a generalized Sweet-Parker model using $\eta^{*}$ instead of $\eta_{\mathrm{Sp}}$ is consistent with the measured reconnection rate in MRX [JI et al., 1998; JI et al., 1999]. The problem then turns to the source of the resistivity enhancement, which is also likely to be closely related to the observed ion heating reported in the next chapter.

In order to determine the plasma resistivity experimentally, consider the toroidal

\footnotetext{
${ }^{6}$ The asymmetry of $V_{\mathrm{R}}$ about zero can be attributed to the slight motion $(\approx 2 \mathrm{~km} / \mathrm{s})$ of the entire reconnection sheet toward the axis of symmetry (due to the force of the equilibrium field).
} 


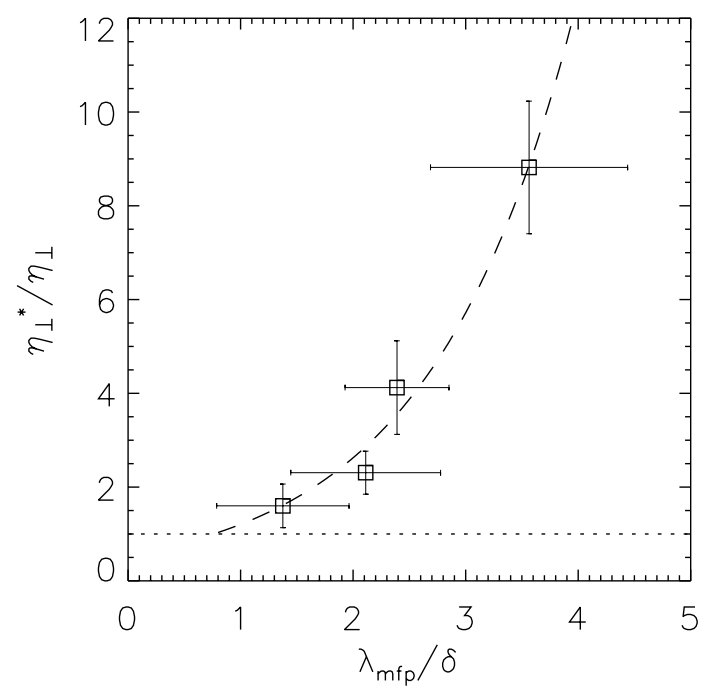

Figure 3.6: Ratio of measured resistivity to classical resistivity, $\eta^{*} / \eta_{\mathrm{Sp}}$, versus an inverse collisionality factor, $\lambda_{\mathrm{mfp}} / \delta$, for null-helicity reconnection in hydrogen discharges. Resistivity enhancement rises as plasma becomes more collisionless. Figure first appeared in [Ji et al., 1998].

component of the simple Ohm's law,

$$
E_{\theta}+\left(B_{\mathrm{R}} V_{\mathrm{Z}}-V_{\mathrm{R}} B_{\mathrm{Z}}\right)=\eta j_{\theta}
$$

In resistive MHD, $\eta$ is the classical Spitzer value [SPITzER, 1962] due to Coulomb collisions. At the center of the reconnection region, where both the second and third terms on the LHS vanish, Eq. (3.7) simplifies to

$$
\eta=\frac{E_{\theta}}{j_{\theta}}
$$

This is a convenient expression since it is not necessary to consider the flow velocities, which are more difficult to determine precisely. Outside the reconnection region, $j_{\theta}$ is small and $E_{\theta}$ is balanced by the $V \times B$ term. The toroidal electric field $E_{\theta}$ is determined using Faraday's law (and invoking axisymmetry),

$$
E_{\theta}=-\frac{1}{2 \pi R} \frac{\partial \psi}{\partial t}
$$




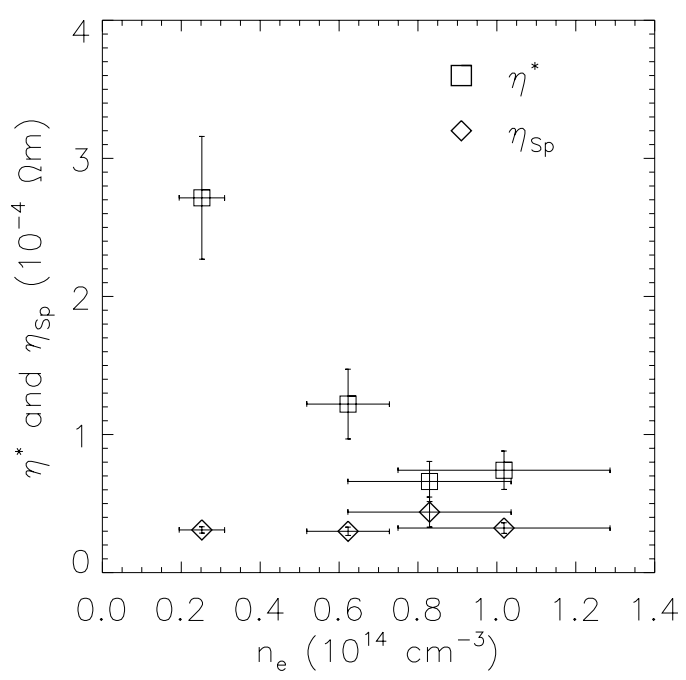

Figure 3.7: Measured and classical resistivities versus density for hydrogen, nullhelicity discharges.

where the poloidal flux $\psi$ is differentiated numerically again using the IDL library routine deriv. The current density is determined using Eq. (3.4). It should be noted that Eq. (3.9) gives only the inductive electric field. However, since the geometry is toroidal, the electrostatic $E_{\theta}$ should be zero over $\theta$, meaning Eq. (3.9) gives the correct average total electric field.

Each term of Eq. (3.8) can be plotted as a function of $R$, as shown in Fig. 3.8 for null-helicity reconnection. The $B_{\mathrm{R}} V_{\mathrm{Z}}$ term in Eq. (3.7) is negligible since $B_{\mathrm{R}} \ll B_{\mathrm{Z}}$ for the pull reconnection geometry [see Fig. 3.1(right)]. Outside the reconnection layer $E_{\theta}$ is indeed balanced by $-V_{\mathrm{R}} B_{\mathrm{Z}}$. However, using Eq. (3.8) and the known values of $E_{\theta}$ and $j_{\theta}$ in the center of the reconnection region, it is seen that $\eta_{\perp}^{*} \equiv$ $E_{\theta} / j_{\theta} \approx 2 \eta_{\perp}$ in this discharge (shot 3444), leading to a factor of two discrepancy in the simple Ohm's law inside the layer. The perpendicular classical resistivity $\eta_{\perp}$ is the relevant quantity for comparison in null-helicity reconnection since the current in this case is perpendicular to the field. ${ }^{7}$ The resistivity enhancement in co-helicity hydrogen discharges is generally about 2, almost within error bar of being classical.

Although $\eta_{\perp}^{*}$ is not a rigorous determination of the true plasma resistivity (because the true plasma resistivity need not be a scalar relationship between $E_{\theta}$ and

\footnotetext{
${ }^{7}$ A detailed calculation including profile effects is given in [KULSRUd, 1997].
} 


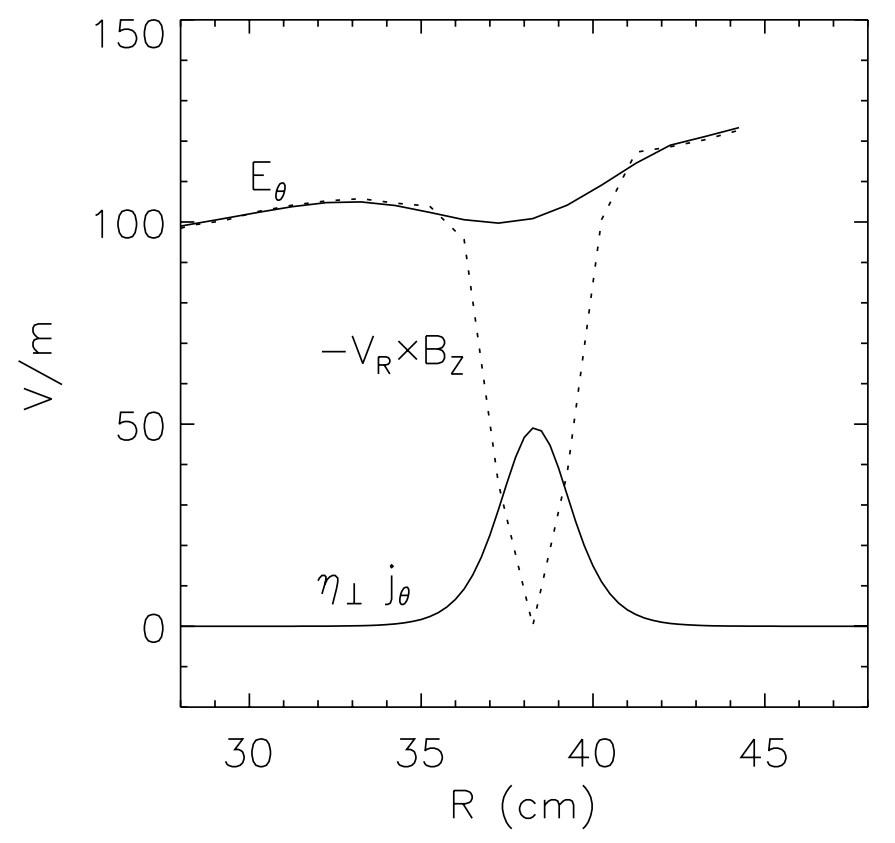

Figure 3.8: Radial profile of the terms in the simple Ohm's law (shot 3444, $t=$ $266 \mu \mathrm{s}$ ), showing that classical resistivity is a factor of two too small to account for the measured $E_{\theta}$ and $j_{\theta}$ inside the current sheet. The relative errors in the curves are about $10 \%$, mostly due to error propagation of measurement uncertainty in $B_{\mathrm{Z}}$.

$\left.j_{\theta}\right)$, it still in some sense represents an "effective" plasma resistivity. Equation (3.7) is valid only for resistive MHD with $\eta_{\mathrm{Sp}}$, and wave-particle interactions and particle inertial effects are not included in Eq. (3.7). The effective resistivity $\eta^{*}$ is a good indicator of the nature of the dominant physics in the reconnection region, i.e. classical Coulomb processes versus non-classical dissipation mechanisms.

\subsection{Magnetic energy dissipation}

The first step to understanding the energy conversion process is to determine how much magnetic energy is dissipated due to reconnection. This can be understood through Poynting's theorem,

$$
-\frac{1}{\mu_{0}} \nabla \cdot(\mathbf{E} \times \mathbf{B})=\frac{\partial}{\partial t}\left(\frac{B^{2}}{2 \mu_{0}}\right)+\mathbf{E} \cdot \mathbf{j},
$$


in which the displacement current term is neglected. The LHS is the Poynting flux, which represents the energy per unit volume carried by the magnetic field into the reconnection region plus the work done per unit volume by the incoming magnetic flux. The first term on the RHS represents the build-up or decay of magnetic field energy density, and the second term is the power dissipation per unit volume due to reconnection. The $\mathbf{E} \cdot \mathbf{j}$ term is the total power per unit volume available due to reconnection to accelerate and heat plasma particles.

The inductive electric field associated with the incoming magnetic flux is balanced by a relative drift between ions and electrons, $\mathbf{V}_{\mathrm{i}}-\mathbf{V}_{\mathrm{e}}=\mathbf{j} /$ ne. However, classical (such as Coulomb collisions) and non-classical (such as wave-particle interactions or inertial effects) remove momentum from the ions and electrons, dissipating the current and also possibly thermalizing the particles accelerated by the electric field. Thus the $\mathbf{E} \cdot \mathbf{j}$ term represents the work done per unit time and volume by the reconnection electric field in sustaining the current, i.e. magnetic field energy is effectively converted to particle kinetic energy.

Taking the dot product of Ohm's law, $\mathbf{E}+\mathbf{v} \times \mathbf{B}=\eta \mathbf{j}$, with $\mathbf{j}$ and using a vector identity, it can be seen that the dissipated magnetic energy consists of two components,

$$
\mathbf{E} \cdot \mathbf{j}=\eta j^{2}+\mathbf{v} \cdot(\mathbf{j} \times \mathbf{B}) .
$$

The LHS is the total available power density due to reconnection. The first term on the RHS is power dissipation in the current sheet, or Ohmic heating in the classical picture. The second term on the RHS is acceleration due to magnetic field pressure and tension, as well as the work done by the magnetic field. Thus, energy conversion due to reconnection can be distributed between the two components. In the SweetParker model, ions can be accelerated due to pressure buildup and field line tension (which was actually neglected in the model) in the layer, and then thermalize due to viscosity. There are also many possible mechanisms for ion heating not included in the MHD description. Already mentioned was simple particle acceleration in the current sheet followed by classical or non-classical collisions. Another mechanism is the acceleration and subsequent mixing of incoming ions due to ambipolar electric fields arising from two-fluid effects [SHAY et al., 1998].

The total energy released due to reconnection in volume $V$ and from time $t_{1} \rightarrow t_{2}$ 
can be calculated as follows:

$$
W_{\text {rec }}=\int_{t_{1}}^{t_{2}} \int_{V} \mathbf{E} \cdot \mathbf{j} d^{3} V d t \approx \int_{t_{1}}^{t_{2}} \int_{V} E_{\theta} j_{\theta} d^{3} V d t .
$$

Since accurate measurement of $j_{\theta}$ for narrow current sheets (null-helicity) requires the $0.5 \mathrm{~cm}$ spatial resolution of the $1-\mathrm{D}$ probe, which is normally situated at $Z=$ $0 \mathrm{~cm}$, the determination of $W_{\text {rec }}$ for null-helicity reconnection is limited also to $Z=0 \mathrm{~cm}$. However, as evident from the contour plots of $\psi$, variation in the $Z$ direction between $Z= \pm 5 \mathrm{~cm}$ is small, and therefore $W_{\text {rec }}$ is not strongly dependent on $Z$. The reconnected field energy $W_{\text {rec }}$ can be compared to the average plasma flow and thermal energies in order to create a detailed energy budget of the reconnection process, as presented in Ch. 4.

\subsection{Highlight of MRX physics results}

Based on the experimental measurements and analysis techniques described in previous sections, several reconnection physics topics have been addressed on MRX over the past four years. This section will summarize these results, which all either have been published or have been submitted for publication. This section is not meant to be comprehensive or exhaustive, as it is not the central topic of the dissertation. However, these results aid the interpretation of ion heating and acceleration measurements, and thus the inclusion of this summary is appropriate.

\subsubsection{Structure of the reconnection region}

The results in this sub-section appear in [YAMADA et al., 1997b]. Prior to this work, the detailed structure of the reconnection layer had not been studied experimentally in plasma in which the MHD approximation was valid globally. This study focused particularly on the effects of an out-of-plane, toroidal magnetic field $B_{\theta}$ on both qualitative and quantitative features of the reconnection layer. A prime motivation for this study was the fact that many influential theoretical reconnection models are 2-D and do not include the effects of an out-of-plane field. Therefore, experimental results are important for determining the applicability of such models to real reconnection events, which generally feature three-component field reconnection. 
(a)

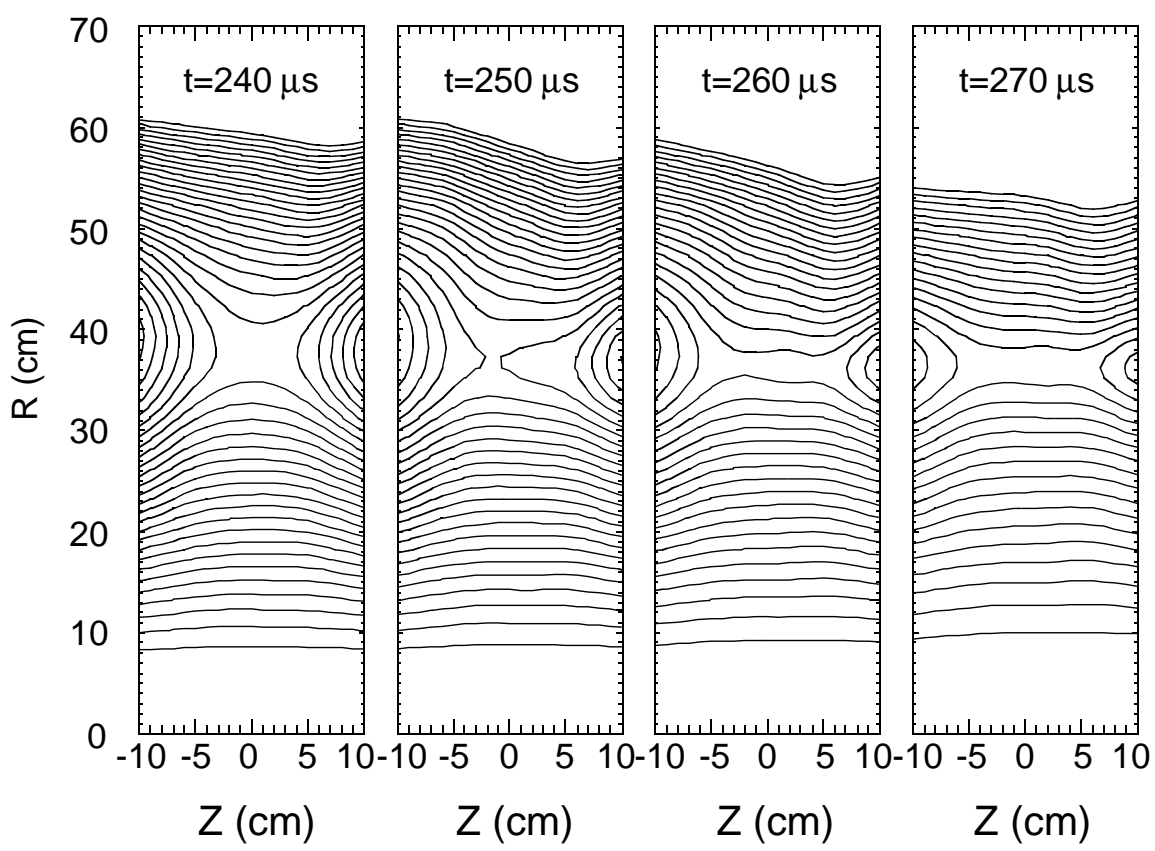

(b)

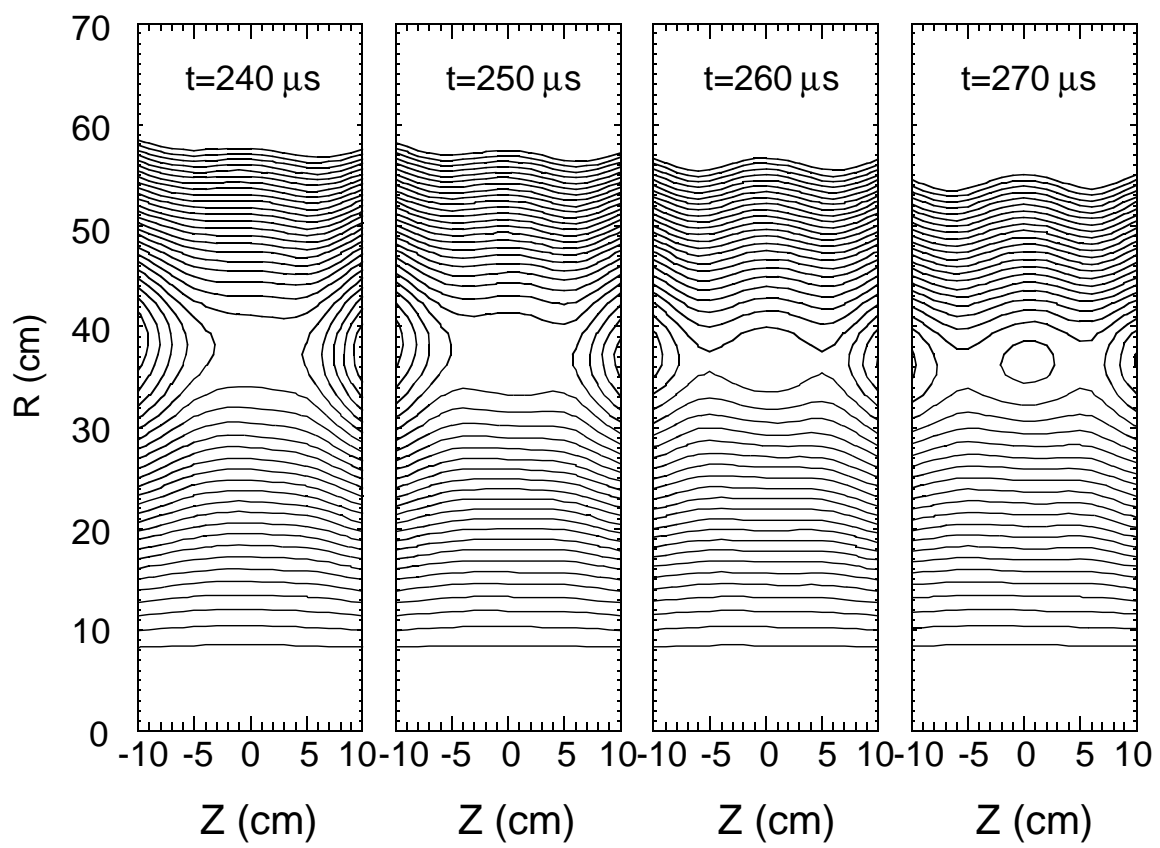

Figure 3.9: Contour plots of the poloidal flux function $\psi$ for (a) null-helicity and (b) co-helicity reconnection, showing a clear qualitative difference in the shapes of the reconnection regions. This figure appeared in [YAMADA et al., 1997b]. 
Using the 90-channel magnetic probe array, the time evolution of the global magnetic field topology was characterized in detail for both null-helicity (no $B_{\theta}$ ) and co-helicity (finite $B_{\theta}$ ) reconnection. All three components of $\mathbf{B}$ were measured in one $R-Z$ plane by scanning the 90 -channel probe in $R$ over multiple shots. The poloidal flux $\psi$ was calculated (see Sec. 3.1) for both cases. Contour plots of $\psi$, as shown in Fig. 3.9, concisely illustrate the key findings of these experiments.

The shape of the reconnection layer (or diffusion region) is seen to depend markedly on the presence of $B_{\theta}$. The classical "double-Y" shape, as postulated by Sweet and Parker, appears in null-helicity reconnection. In this case, the halfthickness $\delta$ of the layer narrows to 1 or $2 \mathrm{~cm}$, which is on the order of $\rho_{\mathrm{i}}$ (defined at the "knee" of the field profile) and also equivalently $c / \omega_{\mathrm{pi}}$ (defined at the center of the reconnection layer). ${ }^{8}$ In contrast, a much thicker layer with an O-point appears in the co-helicity case, eventually growing into a spheromak configuration. Also, it was found that the reconnection speed (at which plasma and magnetic flux enter the reconnection region) was up to three times faster in the null-helicity case than the co-helicity case, confirming a similar finding in merging spheromak experiments reported in [YAMADA et al., 1990b].

The differences between null-helicity and co-helicity reconnection described above are consistent with the effects introduced by finite $B_{\theta}$. First, the toroidal field pressure in the co-helicity makes the plasma effectively less compressible. This in part explains why $\delta$ is much smaller for null-helicity reconnection, in which the plasma is quite compressible. The incompressibility of the plasma in co-helicity reconnection also slows down the inflow of plasma and frozen-in flux, which piles up outside the reconnection layer. This is evident by comparing the density of contour lines in Fig. 3.9 for the two cases. Furthermore, the presence of $B_{\theta}$ introduces an absolute minimum- $B$ configuration, in which case the current channel is stabilized, allowing the O-point to grow to a macroscopic scale size. The presence of the O-point for co-helicity reconnection and the absence of the O-point for null-helicity reconnection both have been reproduced in an MHD simulation [WATANABE et al., 1999] and the results interpreted using a Taylor state [TAYLOR, 1974; TAYLOR, 1986] analysis.

\footnotetext{
${ }^{8}$ This equivalence results from the plasma having a $\beta^{*}$ of unity, where $\beta^{*}$ is defined as the plasma thermal pressure in the reconnection layer divided by the magnetic field pressure at the edge of the layer.
} 
These findings share some common features with the interaction of the solar wind with the Earth's magnetosphere. Space satellite observations at the dayside magnetopause show that solar winds with southward magnetic field reconnect faster with the Earth's (northward) dipolar field than do northward magnetic fields. ${ }^{9}$ These two situations are somewhat analogous to null-helicity and co-helicity reconnection in MRX, respectively.

It is clear that the third field component introduces important effects in the reconnection process. More subtle consequences, however, still need to be studied. One key example is the fact that the reconnection current is a combination of parallel and perpendicular current in the co-helicity case, while it is entirely perpendicular in the null-helicity case. This could have ramifications in the microphysics inside the layer, which determines the plasma resistivity and ultimately the reconnection rate and plasma heating mechanisms. In fact, as will be shown in Ch. 4, null-helicity and co-helicity reconnection result in significantly different ion temperatures.

\subsubsection{Verification of a generalized Sweet-Parker model}

The results in this sub-section appear in [JI et al., 1998; Ji et al., 1999]. For forty years, the seminal MHD reconnection model of Sweet [SwEET, 1958] and Parker [PARKER, 1957] has, on the one hand, been the foundation for countless reconnection theories and, on the other hand, been dismissed for predicting a reconnection rate believed to be orders of magnitude too slow to explain solar flare energy release. In all this time, the details and applicability of the model had not been studied experimentally, which motivated the work described here.

The Sweet-Parker model is a 2-D, steady-state, incompressible fluid theory for reconnection. The reconnection geometry is postulated to consist of an outer region, in which ideal MHD is valid and magnetic flux is frozen into the plasma fluid, and a rectangular reconnection region, in which ideal MHD breaks down and resistive diffusion becomes important. Using the continuity equation and force balance along and across the reconnection layer, it is straightforward to derive a normalized

\footnotetext{
${ }^{9}$ For a list of references, see [KIVELSON and Russell, 1995].
} 
reconnection rate of

$$
\frac{V_{\mathrm{rec}}}{V_{\mathrm{A}}}=\sqrt{\frac{\eta}{\mu_{0} L V_{\mathrm{A}}}} \equiv \frac{1}{\sqrt{S}} .
$$

The derivation assumes equal plasma pressures in the upstream and downstream regions. If the Spitzer value is used for $\eta$, then typical values for $S$ are $10^{10}$ or higher in solar and space plasmas, yielding unacceptably slow reconnection rates. Due to the discrepancy, the Sweet-Parker model, although generally considered to be a useful framework for reconnection, was considered to be incorrect for realistic reconnection scenarios, and attention turned to other models, such as Petschek's slow shock model [PETSCHEK, 1963], which yield faster reconnection rates. However, MRX experimental results suggest that the Sweet-Parker model may yet be applicable to reconnection in nature.

Since all the necessary parameters are measured in MRX, and a quasi 2-D and steady-state reconnection layer can be generated, as shown in the previous subsection, the Sweet-Parker model could be tested experimentally for the first time. A straightforward application of Eq. (3.13) showed that the measured normalized reconnection rate did not scale with $1 / \sqrt{S}$. The next step was to check the assumptions in the Sweet-Parker model one by one. First, and most importantly, it was found that the effective plasma resistivity, $\eta^{*} \equiv E_{\theta} / j_{\theta}$, was enhanced over the appropriate Spitzer value $\eta_{\mathrm{Sp}}\left(=\eta_{\perp}\right.$ for null-helicity and $\eta_{\|}$for co-helicity) by up to a factor of ten for cases where $\lambda_{\operatorname{mfp}} / \delta \gg 1$, usually in null-helicity discharges with low initial gas pressure. Second, the plasma was compressible in null-helicity discharges, allowing a higher inflow speed since mass could be accumulated in the reconnection layer during the time of interest. Third, the downstream plasma pressure was higher than the upstream pressure, reducing the outflow speed and altering the force balance along the layer. Including these three effects, a new generalized reconnection rate, analogous to Eq. (3.13) can be derived:

$$
\frac{V_{\mathrm{rec}}}{V_{\mathrm{A}}}=\sqrt{\frac{\eta^{*}}{\mu_{0} L V_{\mathrm{A}}} \frac{1+L \dot{n} / n V_{\text {out }}}{V_{\mathrm{A}} / V_{\text {out }}}} \equiv \frac{1}{\sqrt{S_{\text {eff }}}},
$$

where $V_{\text {out }}$ is the downstream plasma outflow speed. The measured $V_{\text {rec }} / V_{\mathrm{A}}$ for both null-helicity and co-helicity reconnection fit very well to Eq. (3.14), as shown 
in Fig. 3.10. The excellent agreement suggests that this generalized Sweet-Parker

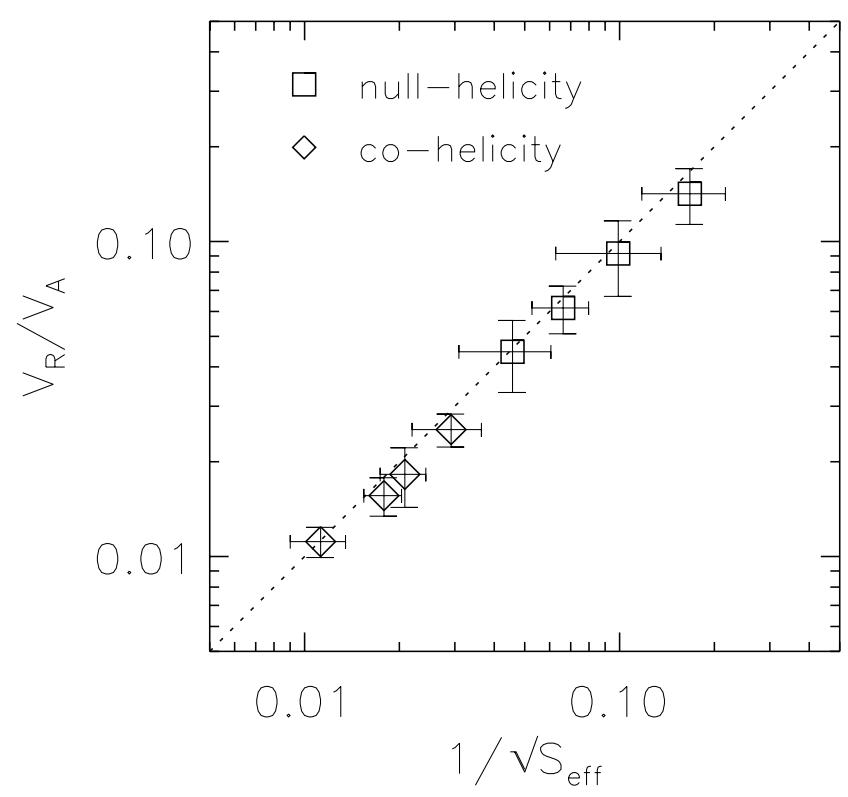

Figure 3.10: Normalized reconnection rate versus generalized Sweet-Parker model parameter $S_{\text {eff }}^{-1 / 2}$.

model, which takes into account enhanced (non-classical) resistivity, plasma compressibility, and finite downstream pressure, may be applicable to quasi 2-D, steadystate reconnection events in nature.

A possible interpretation of these results is that the basic Sweet-Parker formulation is not incorrect, but rather it is the assumption of classical resistivity which is inapplicable. In reality, non-classical effects can enhance the resistivity and make $S_{\text {eff }}$ many orders of magnitude smaller than $S$, perhaps leading to fast enough reconnection rates to explain solar flares. The logical next step is to study what mechanisms determine the enhanced, non-classical resistivity in MRX and how the resistivity scales in even more collisionless regimes (higher $S$ ). 


\subsubsection{Measurement of a Harris-like neutral sheet profile}

These results have been submitted for publication in [YAMADA et al., 2000]. An oftused equilibrium model for studying the reconnection layer (or "neutral sheet"), ${ }^{10}$ especially in numerical simulations, is a collisionless current sheet model known as the "Harris sheet" [HARRIs, 1962]. Detailed field and pressure profile measurements on MRX agree remarkably well with the Harris profiles, despite the fact that the Harris theory does not include reconnection since there is no dissipation. In the present work, the Harris theory is modified slightly to account for $T_{\mathrm{i}} \neq T_{\mathrm{e}}$, and the modified sheet thickness $\delta$ is seen to agree with the data.

The Harris collisionless current sheet, generalized to include $T_{\mathrm{i}} \neq T_{\mathrm{e}}$, is analyzed by solving the 1-D, steady-state Vlasov-Maxwell system of equations. The particle distribution function is

$$
f=n_{0}\left(\frac{m}{2 \pi T}\right)^{3 / 2} \exp \left\{-\frac{m\left[v_{x}^{2}+\left(v_{y}-V\right)^{2}+v_{z}^{2}\right]}{2 T} \pm \frac{e\left(V A_{y}-\phi\right)}{T}\right\},
$$

where $T=T_{\mathrm{e}}\left(T_{\mathrm{i}}\right)$ is the electron (ion) temperature and $V=V_{\mathrm{e}}\left(V_{\mathrm{i}}\right)$ is the electron (ion) drift speed in the current direction $y$. Since the argument of the exponential can be written as $\left(-W+p_{y} V-m V^{2} / 2\right) / T$, then $f$ is a function of the constants of the motion and therefore a solution of the Vlasov equation. Harris realized that this shifted Maxwellian is the most natural solution. Here, Harris' assumptions $T_{\mathrm{e}}=T_{\mathrm{i}}$ and $V_{\mathrm{i}}=-V_{\mathrm{e}}$ have been relaxed. Substituting Eq. (3.15) into the Vlasov-Maxwell system results in the Harris profile solutions:

$$
\begin{aligned}
B_{z} & =-B_{0} \tanh \left(\frac{x}{\delta}\right) \\
j_{y} & =\frac{B_{0}}{\mu_{0} \delta} \operatorname{sech}^{2}\left(\frac{x}{\delta}\right) \\
p & =n_{0}\left(T_{\mathrm{e}}+T_{\mathrm{i}}\right) \operatorname{sech}^{2}\left(\frac{x}{\delta}\right),
\end{aligned}
$$

\footnotetext{
10 "Neutral" refers to magnetically neutral, where the magnitude of the field goes to zero, as in null-helicity discharges in MRX.
} 
where $B_{0}^{2} /\left(2 \mu_{0}\right)=n_{0}\left(T_{\mathrm{e}}+T_{\mathrm{i}}\right)$. The current sheet thickness $\delta$ is given by

$$
\delta=\frac{c}{\omega_{\mathrm{pi}}} \frac{\sqrt{2\left(T_{\mathrm{e}}+T_{\mathrm{i}}\right) / m_{\mathrm{i}}}}{V_{\mathrm{i}}-V_{\mathrm{e}}}=\frac{c}{\omega_{\mathrm{pi}}} \frac{\sqrt{2} V_{\mathrm{s}}}{V_{\mathrm{dr}}},
$$

where $V_{\mathrm{s}} \equiv \sqrt{\left(T_{\mathrm{e}}+T_{\mathrm{i}}\right) / m_{\mathrm{i}}}$ and $V_{\mathrm{dr}} \equiv V_{\mathrm{i}}-V_{\mathrm{e}}$ is the relative drift between ions and electrons. The original Harris solution can be recovered by setting $T_{\mathrm{e}}=T_{\mathrm{i}}=T$ and $V_{\mathrm{i}}=-V_{\mathrm{e}}=V$ in Eq. (3.19) to yield $\delta=\left(c / \omega_{\mathrm{pi}}\right)\left(\sqrt{T / m_{\mathrm{i}}} / V\right)$.

The solutions in Eqs. (3.16)-(3.19) are compared to the experimental data. Radial profiles of the magnetic field, current density, and plasma pressure across the neutral sheet are shown in Fig. 3.11. The $T_{\mathrm{i}}$ component of the plasma pressure is estimated from global Doppler spectroscopy to be roughly twice to three times the $T_{\mathrm{e}}$ component. Note the excellent agreement between $B_{\mathrm{Z}}$ data and the hyperbolic tangent fit function. The current sheet thickness $\delta$ is seen to scale with $c / \omega_{\mathrm{pi}}$

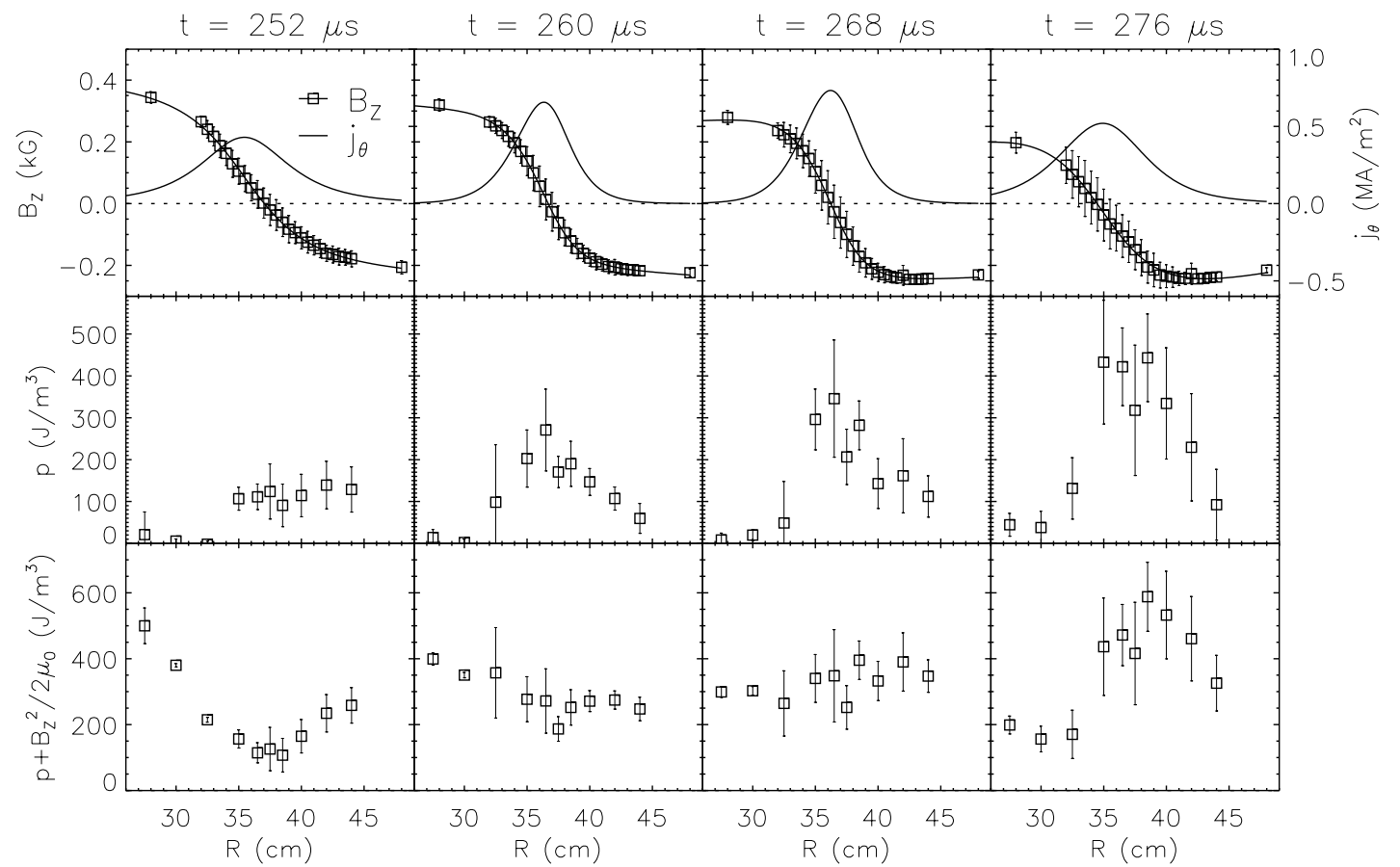

Figure 3.11: Radial profiles of $B_{\mathrm{Z}}$, plasma pressure $n\left(T_{\mathrm{e}}+T_{\mathrm{i}}\right)$, and the sum of plasma and magnetic pressures. Ion temperature is estimated from global Doppler spectroscopy. Pressure balance is maintained during the main part of pull reconnection. This figure is to appear in [YAMADA et al., 2000]. 
and to agree well with the prediction of the modified Harris theory, as shown in Fig. 3.12. The scaling of $\delta$ with $c / \omega_{\text {pi }}$ may be suggestive of two-fluid effects becoming

(a)
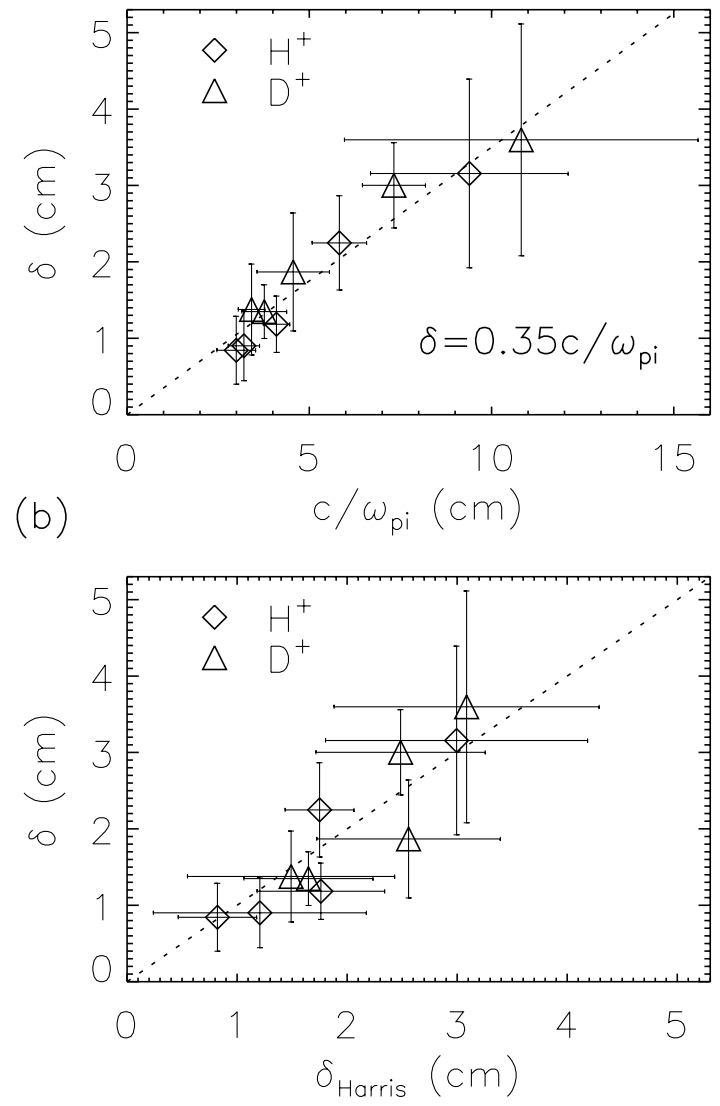

Figure 3.12: The sheet thickness $\delta$ scales with $c / \omega_{\mathrm{pi}}$ and agrees with the predicted thickness of the modified Harris solution, Eq. (3.19). This figure is to appear in [YAMADA et al., 2000].

important, which has been demonstrated in a simulation [SHAY et al., 1998].

As suggested earlier, the agreement between experiment and theory in this case might be considered suprising since the Harris theory does not include the effects of reconnection. However, upon further examination, this may not be surprising. In realistic reconnection current sheets, the dissipation which leads to steady-state reconnection is exactly balanced by energy supplied by the incoming field. As long as the equilibrium across the layer can be maintained, then the Harris solution 
can still be an applicable solution. Pressure balance is seen to exist during pull reconnection in the third row of Fig. 3.11. Because the shifted Maxwellian velocity distribution used in Harris' theory satisfies the full Fokker-Planck equation, it also appears to be the natural state for a steady-state reconnecting plasma.

However, deviations from the Harris model still exist. Measured floating potential radial profiles suggest a plasma potential which decreases monotonically from $V \approx 0 \mathrm{~V}$ (machine ground) at $R=30 \mathrm{~cm}$ to $V \approx-100 \mathrm{~V}$ at $R=45 \mathrm{~cm}$ (see Sec. 4.2.2), in contrast to the Harris solution. The measured potential would give rise to a radial electric field and consequently an $E \times B$ rotation that could modify the expected diamagnetic toroidal drift speeds $V_{\mathrm{i}}$ and $V_{\mathrm{e}}$. This point is discussed further in Sec. 4.2.2 and is also currently under further investigation.

\subsection{Summary}

In this chapter, the experimental characterization of the reconnection process in MRX was given a thorough treatment. From raw magnetic probe data sets, the formation and evolution of the global magnetic topology could be visualized, and details about the structure and characteristic length scales of well-characterized reconnection layer were resolved for the first time. Together with the triple Langmuir probe data, a detailed quantitative understanding of the reconnection process in MRX was developed, including investigations of the reconnection rate and how it scales with $S_{\text {eff }}$, the non-classical nature of the plasma resistivity, and the initally surprising agreement of the current sheet profile with the oft-quoted Harris model. Now armed with the capability to produce and thoroughly diagnose the reconnection process, the study of ion heating and acceleration due to reconnection becomes a possibility. 


\section{Chapter 4}

\section{Ion Heating and Acceleration During Reconnection}

$\mathrm{I}$ ON TEMPERATURE AND FLOW measurements during reconnection are presented in this chapter. Conversion of magnetic field energy to ion kinetic and thermal energy via reconnection is invoked frequently but not often characterized in detail, largely due to the limited accessibility of such events in both naturally occurring and laboratory plasmas. The process is often considered as a "black box," in which the input parameters are, for example, hints of magnetic nulls (such as sunspot groups) or measurable magnetic fluctuations (as in RFP's), and the output parameters are energetic particle beams or enhanced global ion heating. The details of the black box, however, are fundamental to basic plasma physics since reconnection is a virtually unavoidable process in any magnetized plasma. Because local reconnection changes global magnetic field topology, previously distinct regions of plasma can mix. A good example of this is the interaction of the solar wind with the Earth's magnetoshere, in which reconnection along the day-side magnetopause and the polar cusp regions allows solar wind plasma to penetrate into the magnetospheric cavity. Detailed understanding of the magnetic energy conversion process could also be the first step toward eventual creative application of such understanding to areas like alternative concept fusion or even minimization and control of confinement-degrading processes in tokamak plasmas, e.g. [VON GOELER et al., 1974].

The goal of the work presented in this chapter is to examine ion heating and acceleration during reconnection in detail by measuring basic quantities needed to 
develop physical understanding. With the aid of a unique laboratory experiment in MRX, it becomes possible to capture cleanly one possible scenario of what reconnection does to a real plasma when magnetic flux is destroyed. Important questions to address include: (1) whether ions are heated, (2) whether energetic flows develop, (3) what fraction of the dissipated magnetic energy is converted to ion energy, and (4) what is the nature of the energy conversion mechanism(s). Because the time scale of the reconnection process in the experiment $(\approx 30 \mu \mathrm{s})$ is much shorter than the characteristic electron-ion energy equilibration time $(\approx 400 \mu \mathrm{s})$, any ion heating is completely isolated from classical ion-electron interactions, leading to easier interpretation of the data. In light of the above statement, really the only available classical ion heating mechanism due to reconnection on such short time scales would be viscous heating by ion flows which may develop due to reconnection. Other possibilities would involve non-classical mechanisms such as wave-particle interactions or collisionless inertial effects.

Until this work, local ion temperature had not been measured in conjunction with a well-characterized reconnection event. Thus, the measurements presented in this chapter represent an important contribution to the problem. The $T_{\mathrm{i}}$ measurements were rigorous in that they were (1) a direct measurement of the majority plasma ion $T_{\mathrm{i}}$, (2) spatially localized, and (3) temporally resolved. All three requirements were satisfied by using a novel, spectroscopy probe (the IDSP, described in Sec. 2.3.3) and a gated-CCD camera in pure helium discharges. Use of the IDSP and gated-CCD camera ensured spatial $(5 \mathrm{~cm})$ and temporal $(10 \mu \mathrm{s})$ resolution, and use of helium discharges gave Doppler broadened spectra of the majority ions (singly ionized He II $4686 \AA$ ). Details of the Doppler broadening analysis is given in Appendix C. It is important to note that all the data presented in this chapter are from helium discharges with the following typical parameters: $n_{\mathrm{e}} \approx 5 \times 10^{13} \mathrm{~cm}^{-3}$ and $T_{\mathrm{e}} \approx 15 \mathrm{eV}$ in the reconnection layer, and $B_{\mathrm{Z}} \approx 250 \mathrm{G}$ at the edge of the layer, all of which remain fairly constant in time during the main pull reconnection phase.

This chapter is organized as follows. Section 4.1 presents ion temperature measurements which show a rise in $T_{\mathrm{i}}$ that is linked to reconnection, both in the sense that the $T_{\mathrm{i}}$ rise is observed only when reconnection is driven, and that the strongest rise in $T_{\mathrm{i}}$ is spatially localized near the reconnection layer. Section 4.2 presents measurements of both downstream and toroidal ion flow $V_{\mathrm{i}}$, showing that flows which 
develop in either direction are sub-Alfvénic in magnitude. Viscous heating is estimated to be small. Section 4.3 gives a budget for the energy conversion process, showing that more than half of the reconnected field energy is converted to ion thermal energy, and that the conversion is predominantly due to non-classical mechanisms. Section 4.4 discusses the relationship between resistivity enhancement with ion heating, and Sec. 4.5 describes some plausible ion heating mechanisms. The chapter concludes with a summary.

\subsection{Identification of ion heating}

The first step of the investigation was to determine (1) whether a rise in $T_{\mathrm{i}}$ was indeed observable and (2) whether any observed rise in $T_{\mathrm{i}}$ could be causally linked to the reconnection process in convincing fashion. This section reports experimental results which answer the two questions above simultaneously and affirmatively.

\subsubsection{Time evolution of ion temperature}

If ions are heated predominantly by the reconnection process, one might expect $T_{\mathrm{i}}$ to increase with time in the reconnection layer when reconnection is driven and $T_{\mathrm{i}}$ to remain constant when reconnection is not driven, provided that all other controllable parameters are unchanged. ${ }^{1}$

With the IDSP situated in the center of the reconnection region $(R=35 \rightarrow$ $40 \mathrm{~cm}$ and $Z=-2.5 \rightarrow 2.5 \mathrm{~cm}$ ), as shown in Fig. 2.16, $T_{\mathrm{i}}$ as a function of time was obtained for four cases: with and without reconnection (defined below) for both null-helicity and co-helicity discharges. Reconnection (pull mode) is driven when the PF current, as shown in Fig. 2.3, is allowed to ramp down after reaching its peak, which induces the requisite $E_{\theta}$ in the plasma from $t \approx 250 \rightarrow 280 \mu \mathrm{s}$,. To suppress reconnection, the PF circuit is shorted out (crowbarred) at the approximate time of peak current ${ }^{2}$ so that the current decays away over hundreds of microseconds and the induced $E_{\theta}$ is much smaller. For more details regarding

\footnotetext{
${ }^{1}$ It should be mentioned, however, that failure to observe a rise in $T_{\mathrm{i}}$ does not preclude ion heating because the observed $T_{\mathrm{i}}$ also depends on the rate of ion heat loss from the volume being sampled.

${ }^{2}$ The PF crowbar time settings were $t=220 \mu$ s for null-helicity and $t=200 \mu$ s for co-helicity.
} 
plasma formation and control, see Sec. 2.2. All other controllable parameters, such as capacitor bank voltage and initial gas pressure, were kept constant. ${ }^{3}$ The $T_{\mathrm{i}}$ time scan is accomplished by advancing the gate trigger timing for the CCD camera over multiple plasma discharges. The duration over which the CCD gate is open is typically $10 \mu \mathrm{s} .{ }^{4}$

\section{Null-helicity}

A substantial rise in $T_{\mathrm{i}}$ in the center of the reconnection layer, from approximately 6 to $17 \mathrm{eV}$, is observed when reconnection is driven, and no rise in $T_{\mathrm{i}}$ is observed when reconnection is not driven, as shown in Fig. 4.1(top) for null-helicity reconnection. ${ }^{5}$ The rise in $T_{\mathrm{i}}$ is strongly correlated with the magnetic energy dissipation rate, as represented by the value of $E_{\theta} j_{\theta}$ in the center of the reconnection layer, shown in Fig. 4.1(middle). For the case in which no reconnection is driven, the dissipation rate is down by a factor of ten, consistent with $T_{\mathrm{i}}$ remaining constant. Taking the time integral of $E_{\theta} j_{\theta}$ gives the dissipated magnetic energy per unit volume at the center of the reconnection layer, as shown in Fig. 4.1(bottom). Note the remarkable agreement in the time evolution of $T_{\mathrm{i}}$ and the dissipated magnetic energy for both cases, but especially for the case with reconnection. These observations are highly suggestive of reconnection being the ion heating mechanism.

The time evolution of $T_{\mathrm{e}}$ is interesting, as shown in Fig. 4.1(top). The $T_{\mathrm{e}}$ is already at $15 \mathrm{eV}$ early in the discharge, likely due to Ohmic heating during plasma formation as well as the push reconnection phase before $t \approx 245 \mu \mathrm{s}$ in which toroidal field is reconnected. The time evolution of $T_{\mathrm{e}}$ starts to diverge around $t \approx 250 \mu \mathrm{s}$ for the two cases of with and without reconnection. It might appear surprising at first that $T_{\mathrm{e}}$ decreases when reconnection is driven and stays constant when reconnection is not driven. However, there are a few possibilities which may explain this observation. First, when reconnection is driven, the reconnection layer is pushed toward smaller values of $R$, which means that the center of the layer

\footnotetext{
${ }^{3}$ Operation conditions: TF $/ \mathrm{PF}$ bank voltages $=13 / 11 \mathrm{kV}$, fill pressure: $6 \mathrm{mT}$, helium gas

${ }^{4}$ It is increased to $20 \mu \mathrm{s}$ near the start and end of the time scans due to limited plasma light.

${ }^{5}$ The initial $T_{\mathrm{i}} \approx 3-5 \mathrm{eV}$ before $t \approx 245 \mu \mathrm{s}$ for both cases is believed to result from reconnection associated with current ramp-up and plasma formation. In Fig. 4.1(top), error bars in the ordinate represent standard deviations in an ensemble of $T_{\mathrm{i}}$ measurements (5-10 discharges), and error bars in the abscissa represent the CCD gate time.
} 


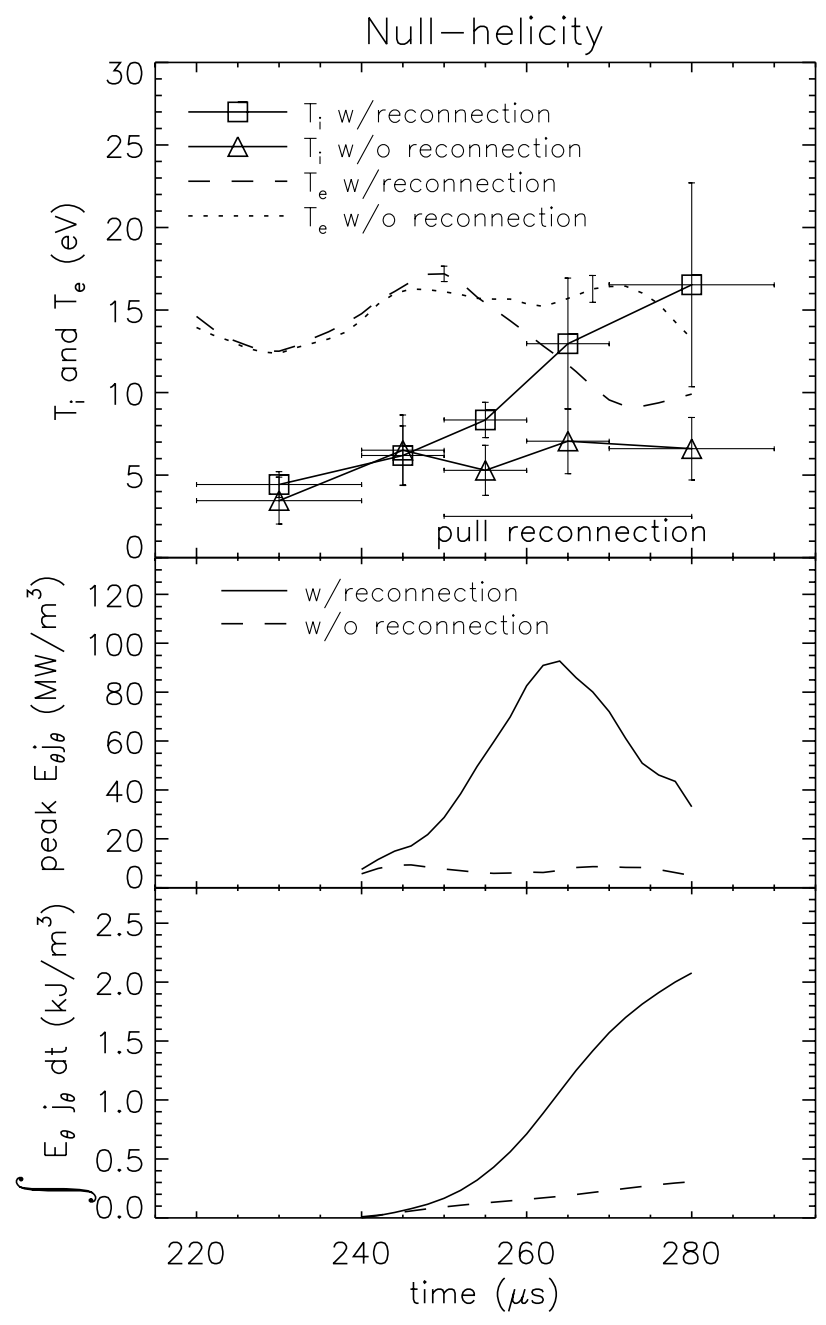

Figure 4.1: Time evolution of (top) $T_{\mathrm{i}}$ and $T_{\mathrm{e}}$, (middle) local heating rate $E_{\theta} j_{\theta}$, and (bottom) dissipated magnetic energy $\int_{240 \mu \mathrm{S}}^{t}\left(E_{\theta} j_{\theta}\right) d t^{\prime}$ per unit volume, all in the center of the reconnection layer for null-helicity discharges (IDSP located at $R=37.5 \mathrm{~cm}$ and $Z=0 \mathrm{~cm}$ ). The rise in $T_{\mathrm{i}}$ is clearly linked to magnetic energy dissipation associated with reconnection. 
with highest $T_{\mathrm{e}}$ can sweep past the stationary triple Langmuir probe. Second, the electron confinement characteristics of the two cases may be very different; this needs further investigation. Finally, the most interesting possibility is that electron energy can be converted to ion energy via non-classical processes which arise only when pull reconnection is driven. This is only speculation, but such a process would be consistent with the $T_{\mathrm{e}}$ measurements.

A direct ion heating mechanism must be operative if reconnection is in fact responsible for the ion heating. "Direct" means that dissipated field energy is converted to ion energy without the need for classical energy exchange with electrons. In these helium discharges, ions cannot be heated classically by the electrons because the ion-electron energy equipartition time is $400 \mu \mathrm{s}$ or more, and furthermore, the energy gained by electrons during reconnection due to Ohmic heating is insufficient in magnitude (see Sec. 4.3.6). One obvious direct ion heating mechanism is classical viscous heating by ion flows accelerated due to reconnection. This method of ion heating would likely scale with increased magnetic energy dissipation (e.g. due to increased magnetic tension force) and possibly explain the good agreement between the $T_{\mathrm{i}}$ rise and dissipated field energy. However, classical viscous heating is estimated later to be insufficient in MRX (see Sec. 4.3.6). This leaves only the possibility of non-classical mechanisms.

It is tempting to read into the direct proportionality between the $T_{\mathrm{i}}$ rise and the dissipated magnetic energy, which suggests that the magnetic energy dissipation rate is linearly related to the ion heating rate. If true, this would imply that the mechanism allowing reconnection to proceed, i.e. some form of non-classical resistivity, is the same mechanism which is heating the ions. Note that in general the two mechanisms do not have to be the same. This interpretation must be taken cautiously because the rise in $T_{\mathrm{i}}$, which is observed, does not have to be proportional to the ion heating rate, which is not observed, due to the likely variation of ion loss rates as the dissipation rate changes.

Prior to using the IDSP, preliminary measurements of $T_{\mathrm{i}}$ were obtained using chord-averaged spectroscopy in null-helicity discharges with slightly different experimental parameters. ${ }^{6}$ The $T_{\mathrm{i}}$ measurements from this work were also taken from Doppler broadening of He II $4686 \AA$ ion emission in helium discharges, but the

\footnotetext{
${ }^{6} \mathrm{TF} / \mathrm{PF}$ voltages $=12 / 10 \mathrm{kV}$, initial gas pressure $8 \mathrm{mT}$
} 
viewing chord (approximately $3 \mathrm{~cm}$ diameter) spanned the entire machine and was tangent to the current sheet at $R=37.5 \mathrm{~cm}$. Shown in Fig. 4.2 are the time evolution of both the chord-averaged Doppler $T_{\mathrm{i}}$ and the local $T_{\mathrm{e}}$ in the reconnection

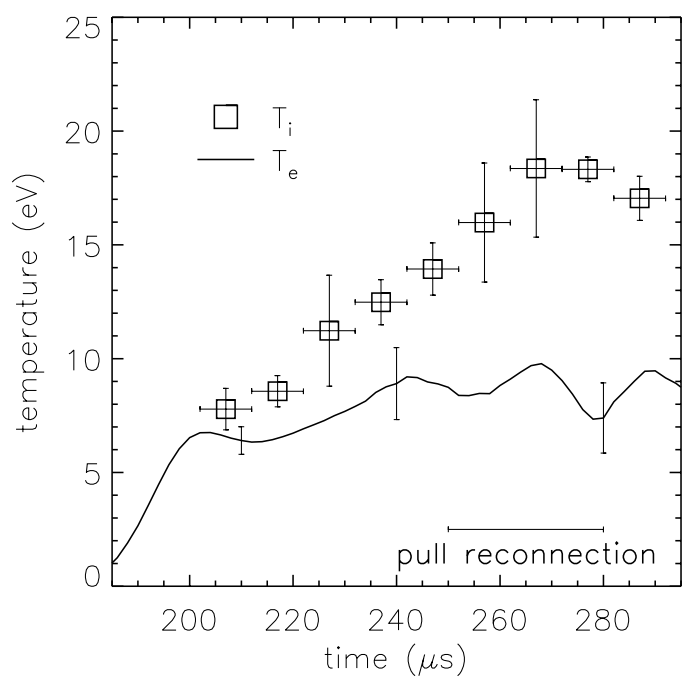

Figure 4.2: Time evolution of chord-averaged Doppler temperature for $T_{\mathrm{i}}$ and local $T_{\mathrm{e}}$ in the reconnection layer. These earlier measurements are consistent with the IDSP results. This figure is included in [YAMADA et al., 2000].

layer (the latter measured by the triple Langmuir probe). Since the chord-averaged spectroscopic data was not Abel-inverted, e.g. according to [BELL, 1997], the $T_{\mathrm{i}}$ values in Fig. 4.2 are likely overestimates. Nevertheless, this data, shown here for completeness, is generally consistent (both in magnitude and time evolution) with the more rigorous measurements of the IDSP.

\section{Co-helicity}

In the co-helicity case, $T_{\mathrm{i}}$ also rises during reconnection, from approximately 3 to $7 \mathrm{eV}$, as shown in Fig. 4.3(top). Again, no rise in $T_{\mathrm{i}}$ is observed if reconnection is not driven. The local magnetic energy dissipation rate $E_{\theta} j_{\theta}$ is shown in Fig. 4.3(middle). In magnitude, it is on average a factor of two smaller than the null-helicity case, consistent with the observed smaller rise in $T_{\mathrm{i}}$. The energy dissipation $E_{\theta} j_{\theta}$ goes through zero at $t=270 \mu$ s due to an unstable plasma "pinching off" from one fluxcore, resulting in a negative $E_{\theta}$ in the measurement area. The dissipated magnetic 


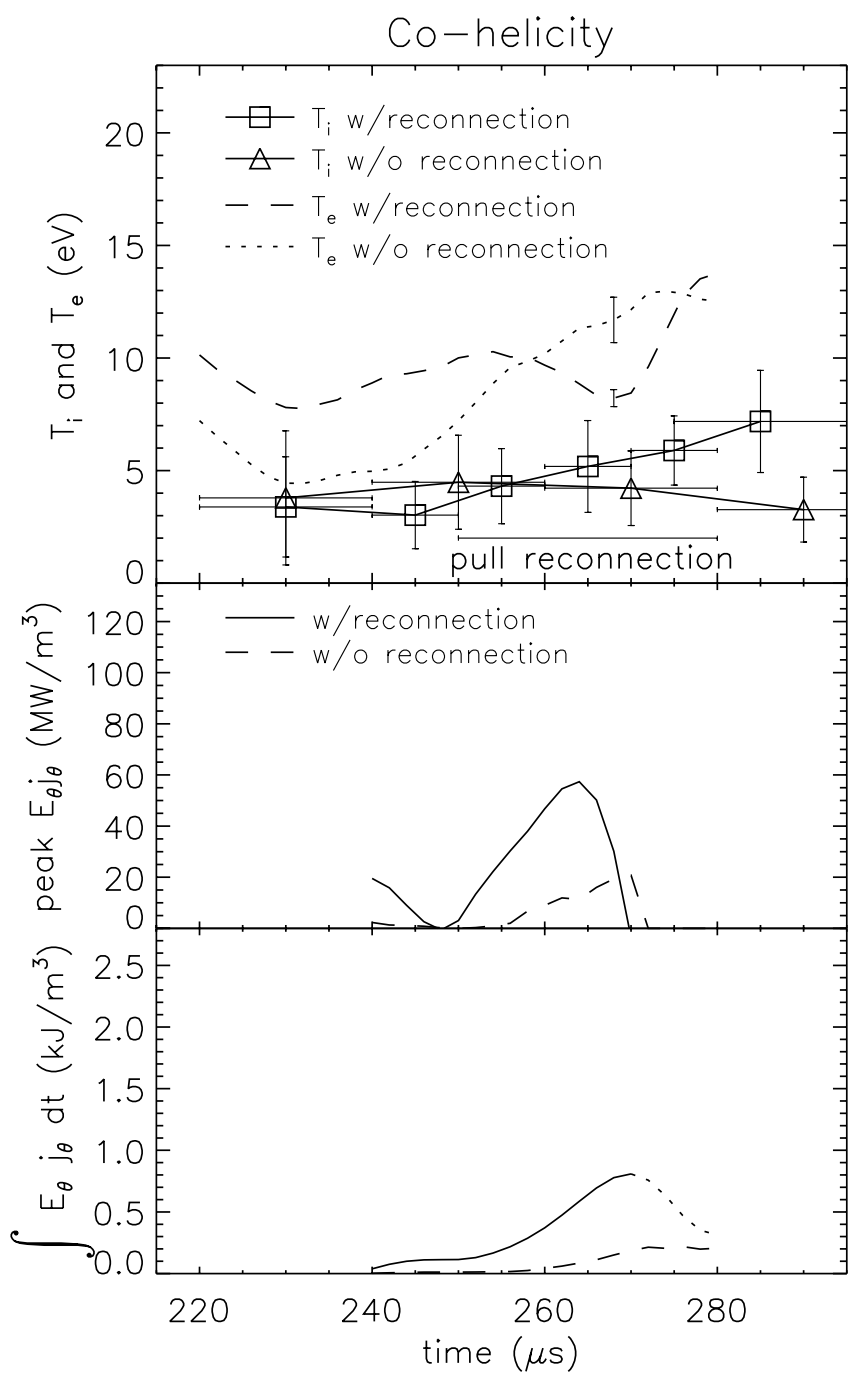

Figure 4.3: Time evolution of (top) $T_{\mathrm{i}}$ and $T_{\mathrm{e}}$, (middle) local heating rate $E_{\theta} j_{\theta}$, and (bottom) energy dissipated $\int_{240 \mu \mathrm{S}}^{t}\left(E_{\theta} j_{\theta}\right) d t^{\prime}$ per unit volume, all in the center of the reconnection layer for co-helicity discharges (IDSP located at $R=37.5 \mathrm{~cm}$ and $Z=0 \mathrm{~cm}$ ). The rise in $T_{\mathrm{i}}$ is much smaller than the null-helicity case, consistent with the smaller dissipated magnetic energy. The reduction of $E_{\theta} j_{\theta}$ to zero at $t=270 \mu \mathrm{s}$ (before the end of the expected pull reconnection phase) is due to an unstable plasma "pinching off" from one flux-core, which also explains the nonphysical decrease in dissipated energy (represented by the dotted line in the bottom panel). 
energy as a function of time is shown in Fig. 4.3(bottom), and the initial rise in $T_{\mathrm{i}}$ is well-correlated to the dissipated magnetic energy. The dotted section of the curve after $t=270 \mu \mathrm{s}$ is a result of the $E_{\theta} j_{\theta}$ term going negative, and this should not be interpreted as part of the main reconnection sequence. The continued rise in $T_{\mathrm{i}}$ after $t=270 \mu \mathrm{s}$ is likely due to uncontrolled reconnection and the improved confinement of the spheromak configuration which forms.

The time evolution of $T_{\mathrm{e}}$, shown in Fig. 4.3(top), for the co-helicity case differs from the null-helicity case. First, $T_{\mathrm{e}} \approx 10 \mathrm{eV}$ for the co-helicity case compared to $15 \mathrm{eV}$ for the null-helicity case. The discussion given previously to account for the difference in $T_{\mathrm{e}}$ after $t \approx 250 \mu \mathrm{s}$ for null-helicity should apply to the co-helicity case also, except in this case the $T_{\mathrm{e}}$ rise at $t=270 \mu \mathrm{s}$ (with reconnection) may be due to the improved confinement of the "pinched-off" spheromak. The difference in $T_{\mathrm{e}}$ before $t \approx 250 \mu \mathrm{s}$ in this case may be attributed to a very early PF crowbar time of $t=200 \mu$ s for suppressing reconnection (compared to $t=220 \mu$ s for null-helicity), although the details need further investigation.

\section{Comparison of null-helicity and co-helicity}

Figure 4.4 shows the key results from null-helicity and co-helicity together. In the null-helicity case, $T_{\mathrm{i}}$ rises by an amount $\Delta T_{\mathrm{i}} \approx 11 \mathrm{eV}$ (from $t=245 \rightarrow 280 \mu \mathrm{s}$ ) and the local dissipated energy at $t=280 \mu \mathrm{s}$ is $2.1 \mathrm{~kJ} / \mathrm{m}^{3}$. In the co-helicity case, which is valid up to $t=270 \mu$ s (due to $E_{\theta}$ going negative), $\Delta T_{\mathrm{i}} \approx 2.5 \mathrm{eV}$ (from $t=245 \rightarrow 270 \mu \mathrm{s}$ ) and the local dissipated energy is $0.8 \mathrm{~kJ} / \mathrm{m}^{3}$ and $t=270 \mu \mathrm{s}$. These numbers indicate that null-helicity reconnection heats ions more effectively since 2.6 times more dissipated energy resulted in 4.4 times rise in $T_{\mathrm{i}}$. Furthermore, the large difference exists despite the fact that co-helicity likely has better ion confinement due to a strong toroidal field. ${ }^{7}$

Stronger ion heating might suggest the increased effect of non-classical dissipation, and this can be investigated with respect to resistivity enhancement over $\eta_{\mathrm{Sp}}$, as discussed in Sec. 3.5. Figure 4.5 shows the time evolution of $E_{\theta}$ and $j_{\theta}$ separately (as well as $E_{\theta} j_{\theta}$ ) for null-helicity and co-helicity reconnection, ${ }^{8}$ from

\footnotetext{
${ }^{7}$ There is evidence for this in that the measured floating potential is always more positive (with respect to machine ground) for co-helicity than for null-helicity (see Figs. 4.11 and 4.12).

${ }^{8}$ The negative values of $E_{\theta}$ and $j_{\theta}$ before $t \approx 248 \mu$ s for co-helicity are due to the end of the push reconnection phase (see Sec. 2.2), in which the induced $E_{\theta}$ is in the other direction. The zero
} 


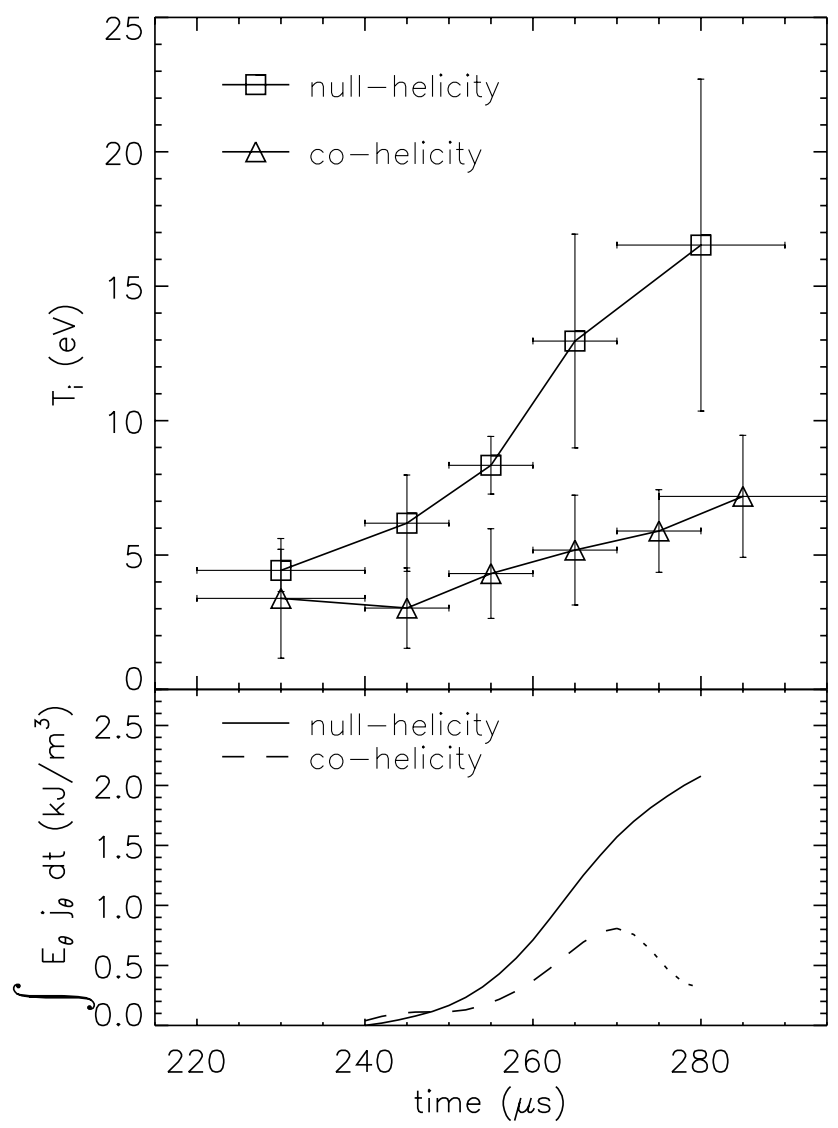

Figure 4.4: Time evolution of (top) $T_{\mathrm{i}}$ with the IDSP placed in the center of the reconnection layer $(R=37.5 \mathrm{~cm}$ and $Z=0 \mathrm{~cm}$ ) and (bottom) dissipated magnetic energy for both null-helicity and co-helicity reconnection.

which a time-averaged effective resistivity, $\eta^{*} \equiv E_{\theta} / j_{\theta}$, can be determined for the two cases. For the null-helicity case (averaging over $t \approx 245 \rightarrow 280 \mu \mathrm{s}$ ), $\eta^{*} \approx(140 \mathrm{~V} / \mathrm{m}) /\left(0.4 \mathrm{MA} / \mathrm{m}^{2}\right) \approx 3.5 \times 10^{-4} \Omega \cdot \mathrm{m}$ and the classical $\eta_{\perp} \approx 2 \times 10^{-5} \Omega \cdot \mathrm{m}$ $\left(T_{\mathrm{e}} \approx 15 \mathrm{eV}\right.$, using $\left.Z_{\mathrm{eff}}=1\right),{ }^{9}$ resulting in an enhancement factor of 18 . For the cohelicity case (averaging over $t \approx 250 \rightarrow 270 \mu \mathrm{s}), \eta^{*} \approx(50 \mathrm{~V} / \mathrm{m}) /\left(0.35 \mathrm{MA} / \mathrm{m}^{2}\right) \approx$ $1.4 \times 10^{-4} \Omega \cdot \mathrm{m}$ and the classical $\eta_{\|} \approx 1.8 \times 10^{-5} \Omega \cdot \mathrm{m}\left(T_{\mathrm{e}} \approx 10 \mathrm{eV}\right.$, using $\left.Z_{\text {eff }}=1\right)$, resulting in an enhancement factor of 8 . In hydrogen discharges, the enhancement

crossing occurs earlier for null-helicity due to the shorter $L / R$ time of the null-helicity plasma. And, as explained previously, the negative $E_{\theta}$ after $t=270 \mu$ s is due to the plasma "pinching off" from one flux-core.

${ }^{9}$ The use of $\eta_{\perp}$ for null-helicity is based on [KuLsRUd, 1997]. 
factor is generally smaller, especially for co-helicity reconnection for which the enhancement is only a factor of two. It is important to note that the higher values of $\eta^{*} / \eta_{\text {Sp }}$ in helium discharges may be due in part to the presence of double-ionized helium as well as double-ionized impurities (since $T_{\mathrm{e}}$ is more than $50 \%$ higher in helium). However, even a $Z_{\text {eff }}=2$, which would be an extreme upper limit in MRX, would still yield enhancement factors of approximately 9 and 4 for null-helicity and co-helicity, respectively. The difference between hydrogen and helium discharges warrants further investigation. The larger resistivity enhancement for null-helicity compared to co-helicity in helium seems to be consistent with the implied stronger heating.

The reduced dissipation rate in co-helicity is due to a factor of two reduction in $E_{\theta}$, which translates to a slower reconnection speed (since reconnection speed is proportional to $E_{\theta}$ ). This is consistent with previous findings that co-helicity reconnection is up to a factor of three slower than null-helicity [YAMADA et al., 1997b; YAMADA et al., 1997a] and counter-helicity reconnection [YAMADA et al., $1990 \mathrm{~b}]$.

\section{Summary}

In these $T_{\mathrm{i}}$ temporal-scan experiments, an increase in $T_{\mathrm{i}}$ during reconnection was indeed identified and causally linked to the presence of reconnection. This finding is a significant result because local ion heating due to reconnection had not been identified before experimentally. In both null-helicity and co-helicity reconnection, $T_{\mathrm{i}}$ increased when reconnection was driven and stayed constant when reconnection was not driven. The $T_{\mathrm{i}}$ increase correlated well with the dissipation of magnetic energy due to reconnection, showing remarkable proportionality with the dissipated field energy in the null-helicity case. Null-helicity reconnection resulted in a disproportionally higher $T_{\mathrm{i}}$ increase compared to the co-helicity case, indicating stronger ion heating for null-helicity. This is consistent with the fact that null-helicity reconnection had a higher value of resistivity enhancement, which suggests the possible role played by non-classical dissipation mechanisms. 


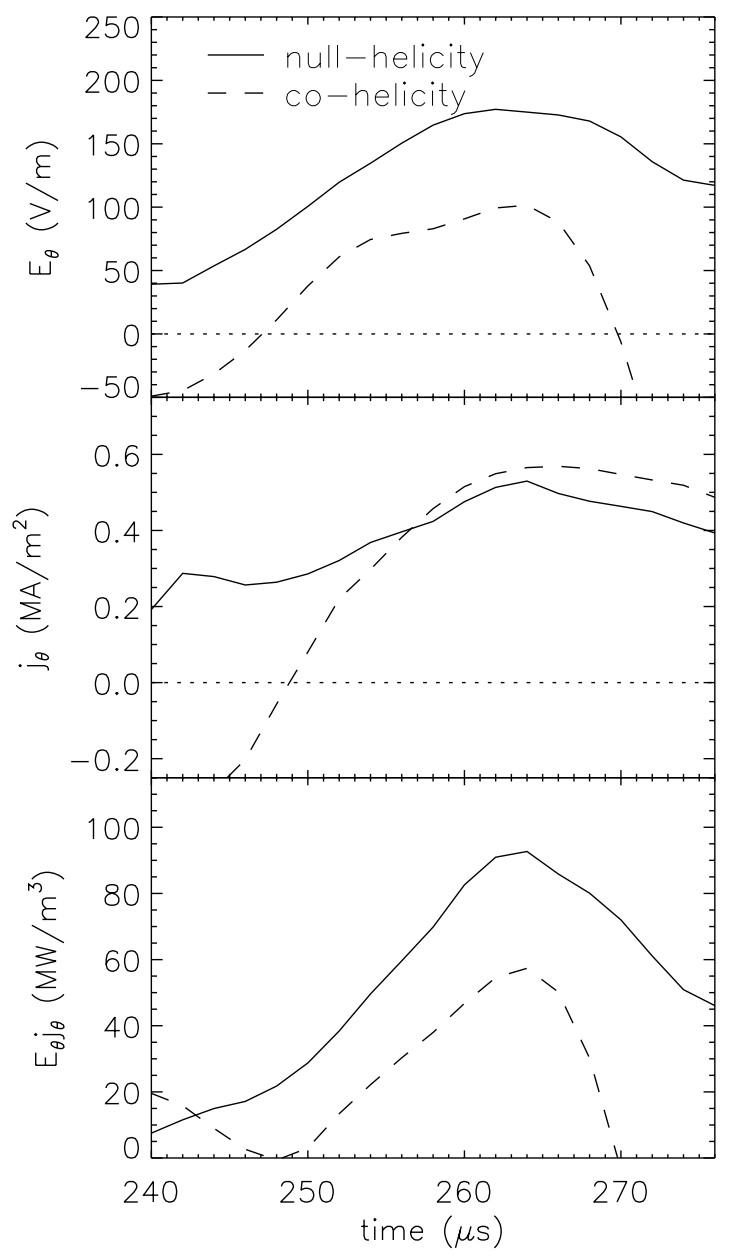

Figure 4.5: Time evolution of (top) $E_{\theta}$, (middle) $j_{\theta}$, and (bottom) $E_{\theta} j_{\theta}$ for nullhelicity and co-helicity, all in the center of the reconnection layer.

\subsubsection{Radial profile of ion temperature rise}

If ions are heated predominantly by the reconnection process, one would also expect the observable rise in $T_{\mathrm{i}}$ to be largest near the reconnection layer $(R \approx 35 \rightarrow 40 \mathrm{~cm})$. Indeed, this was seen experimentally.

To determine the spatial profile of the ion heating, the IDSP was scanned in $R$ for two CCD gate times, $t_{1}=250 \rightarrow 260 \mu$ s and $t_{2}=260 \rightarrow 270 \mu \mathrm{s}$; define $\Delta T_{\mathrm{i}}=T_{\mathrm{i}}\left(t_{2}\right)-T_{\mathrm{i}}\left(t_{1}\right)$. Figure 4.6 shows a peaked profile for $\Delta T_{\mathrm{i}} / T_{\mathrm{i}}\left(t_{1}\right)$ for nullhelicity reconnection, suggesting that ion heating occurred in the vicinity of the reconnection layer. The reconnecting field $B_{\mathrm{Z}}$ profile averaged over the same shots 


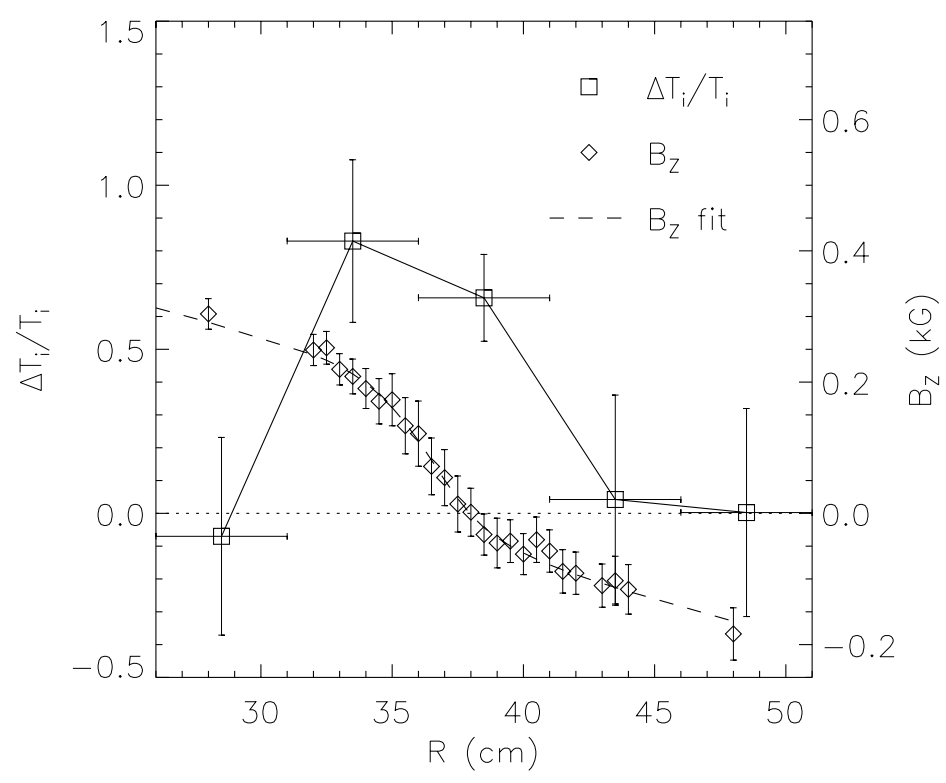

Figure 4.6: Radial profiles of (solid line) the relative rise in $T_{\mathrm{i}}$ from $t=255 \rightarrow 265 \mu \mathrm{s}$ $(R=37.5 \mathrm{~cm}, Z=0 \mathrm{~cm})$ and (dashed line) the reconnecting field $B_{\mathrm{Z}}$ during the same time (also at $Z=0 \mathrm{~cm}$ ) for null-helicity discharges (shots 11614-11659). Rise in $T_{\mathrm{i}}$ is localized near the reconnection layer.

is also shown to indicate the location of the reconnection layer (between the "knees" of the $B_{\mathrm{Z}}$ profile, $R \approx 35 \rightarrow 40 \mathrm{~cm}$ ). Ion temperature rises approximately $75 \%$ in the reconnection layer and not at all elsewhere. Error bars in the ordinate represent one standard deviation in an ensemble of $\Delta T_{\mathrm{i}}$ measurements (approximately 5 discharges at each gate time), and error bars in the abscissa represent the spatial region from which plasma light is collected by the IDSP.

For the co-helicity case, rise in $T_{\mathrm{i}}$ is small compared to the statistical error between shots, and the observed spatial heating profile is essentially flat (within error bars). Better temporal and spatial resolution than what is currently possible is required to reveal a meaningful spatial profile of $\Delta T_{\mathrm{i}} / T_{\mathrm{i}}$ for co-helicity discharges.

In the above experiment, a spatial correlation between the rise in $T_{\mathrm{i}}$ and the location of the reconnection layer was shown. This is further indication that the reconnection process is directly responsible for the observed rise in $T_{\mathrm{i}}$. 


\subsection{Ion flow measurements}

Reconnection is generally expected to accelerate bulk plasma flows. Flows may arise due to large electric fields, $\mathbf{j} \times \mathbf{B}$ forces, and pressure gradients. In TS-3 [ONO et al., 1996; ONO et al., 1997], downstream Alfvénic flows were attributed to $\mathbf{j} \times \mathbf{B}$, specifically the tension force of reconnected field lines. In basic theoretical pictures like the Sweet-Parker model [SwEet, 1958; PARKER, 1957], Alfvénic flows develop due to large pressure gradients between the reconnection layer and the far-away downstream region. Indeed, in the research community, reconnection can hardly be mentioned without the expectation of Alfvénic plasma flows, and one of the conventionally accepted means of ion heating is via viscous damping of energetic flows produced by reconnection. However, measurements of ion flow in MRX indicate that a different scenario is possible, namely that ion heating can occur with sub-Alfvénic flows and small viscous heating.

\subsubsection{Downstream flow}

Ion downstream flow speed profiles were measured locally using a Mach probe, which was calibrated using the IDSP (see Appendix B). The maximum downstream flow $V_{\mathrm{iZ}} \approx 8 \mathrm{~km} / \mathrm{s}$, as shown in Fig. 4.7 for null-helicity reconnection, is equal to $0.2 V_{\mathrm{A}}$, where $V_{\mathrm{A}} \approx 39 \mathrm{~km} / \mathrm{s}\left(n_{\mathrm{e}} \approx 5 \times 10^{13} \mathrm{~cm}^{-3}, B \approx 250 \mathrm{G}\right.$, and mass of helium). The flow speed is also seen to increase linearly from 0 to $8 \mathrm{~km} / \mathrm{s}$ along the layer from $Z=0 \rightarrow 10 \mathrm{~cm}$. The pattern of the flow is consistent with 2$\mathrm{D}$ theoretical reconnection models, e.g. Sweet-Parker, but the magnitude of the flow differs substantially (theoretical models generally predict Alfvénic downstream flows).

The maximum energy density of the measured flow $\left(\rho V_{\mathrm{iZ}}^{2} / 2 \approx 11 \mathrm{~J} / \mathrm{m}^{3}\right.$, using $\left.n_{\mathrm{e}}=5 \times 10^{13} \mathrm{~cm}^{-3}\right)$ is an order of magnitude smaller than the observed ion thermal energy density increase $\left(3 n \Delta T_{\mathrm{i}} / 2 \approx 120 \mathrm{~J} / \mathrm{m}^{3}\right.$, using $n_{\mathrm{e}}=5 \times 10^{13} \mathrm{~cm}^{-3}$ which remains roughly constant in time and $\Delta T_{\mathrm{i}}=10 \mathrm{eV}$ ), implying that the observed ion heating is unlikely to result from thermalization of the outflow. Furthermore, since the flows are stronger at the edges of the layer $(Z= \pm 10 \mathrm{~cm})$, ion heating due to viscosity could not easily explain the observed ion heating in the center of the reconnection region $(Z=0 \mathrm{~cm})$. Estimates of the ion heating due to viscosity 


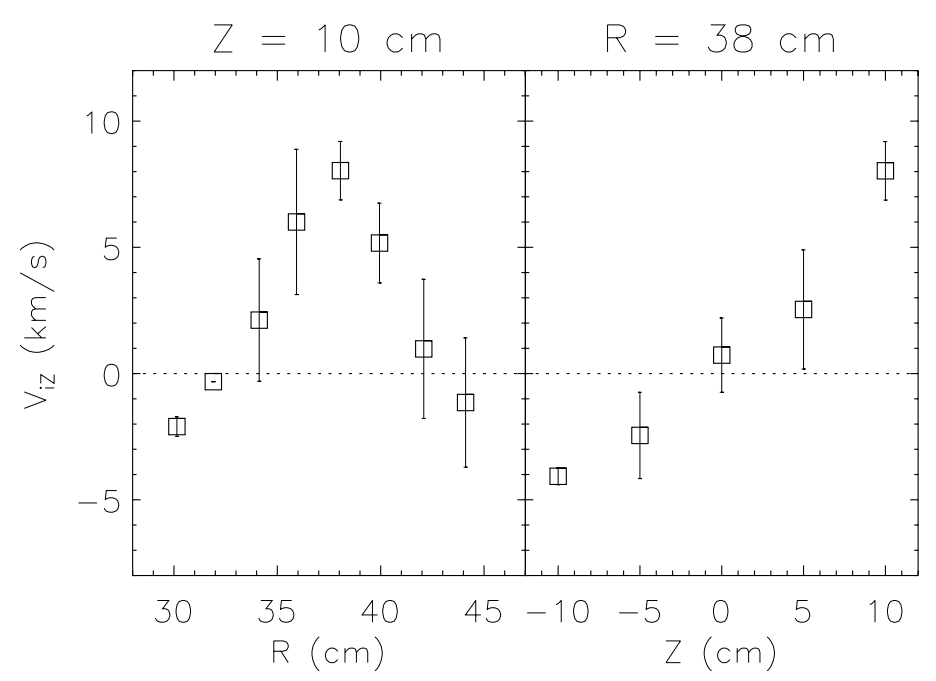

Figure 4.7: Mach probe measurements of $V_{\mathrm{iZ}}$ as a function of $R$ at $Z=10 \mathrm{~cm}$ and as a function of $\mathrm{Z}$ at $R=38 \mathrm{~cm}$ averaged over $t=250 \rightarrow 270 \mu \mathrm{s}$. The magnitude of the flow exiting the reconnection layer is only $20 \%$ of $V_{\mathrm{A}} \approx 39 \mathrm{~km} / \mathrm{s}$, consistent with a non-negligible downstream pressure.

are estimated in Sec. 4.3 and shown to be small. The absence of energetic flows in MRX is an important difference compared to the situation in TS-3 [ONO et al., 1996; ONO et al., 1997], in which ion acceleration to $V_{\mathrm{A}}$ was attributed to the high tension forces of reconnected field lines during the collision of two spheromaks. Also, the downstream pressure in TS-3 was likely to be much lower than the pressure in the reconnection layer. In MRX, ion heating occurs without the occurrence of energetic downstream flows, an important new observation.

The sub-Alfvénic $V_{\mathrm{iz}}$ in MRX is consistent with the buildup of high downstream pressure which reduces the $\nabla p$ force along $Z$, as mentioned in [JI et al., 1998; Ji et al., 1999]. Theoretical models generally do not account for high downstream pressure impeding the flow, and thus ion flows are accelerated to $V_{\mathrm{A}}$. In MRX, high downstream pressure ${ }^{10}$ has been verified by Langmuir probe measurements of $n_{\mathrm{e}}$ and $T_{\mathrm{e}}$ in the downstream region, as shown in Fig. 4.8. However, the $T_{\mathrm{i}} Z$-profile

\footnotetext{
${ }^{10}$ The high downstream pressure in MRX may arise due to fast pressure equalization along field lines which connect the reconnection region to the finite-volume downstream regions near the flux-cores. This is best visualized in Fig. 2.4(c). The thermal transit time of $15 \mathrm{eV}$ electrons along this path is on the order of $1 \mu \mathrm{s}$, meaning that the buildup of downstream pressure due to recirculating electrons over the $20-30 \mu$ s duration of pull reconnection is completely plausible.
} 
was not measured since the IDSP could not be placed there. The $Z$-profile of $n T_{\mathrm{e}}$ is peaked near $Z=0 \mathrm{~cm}$ early in the reconnection phase but it becomes flat later in the reconnection phase, mostly due to a rise in $n_{\mathrm{e}}$.
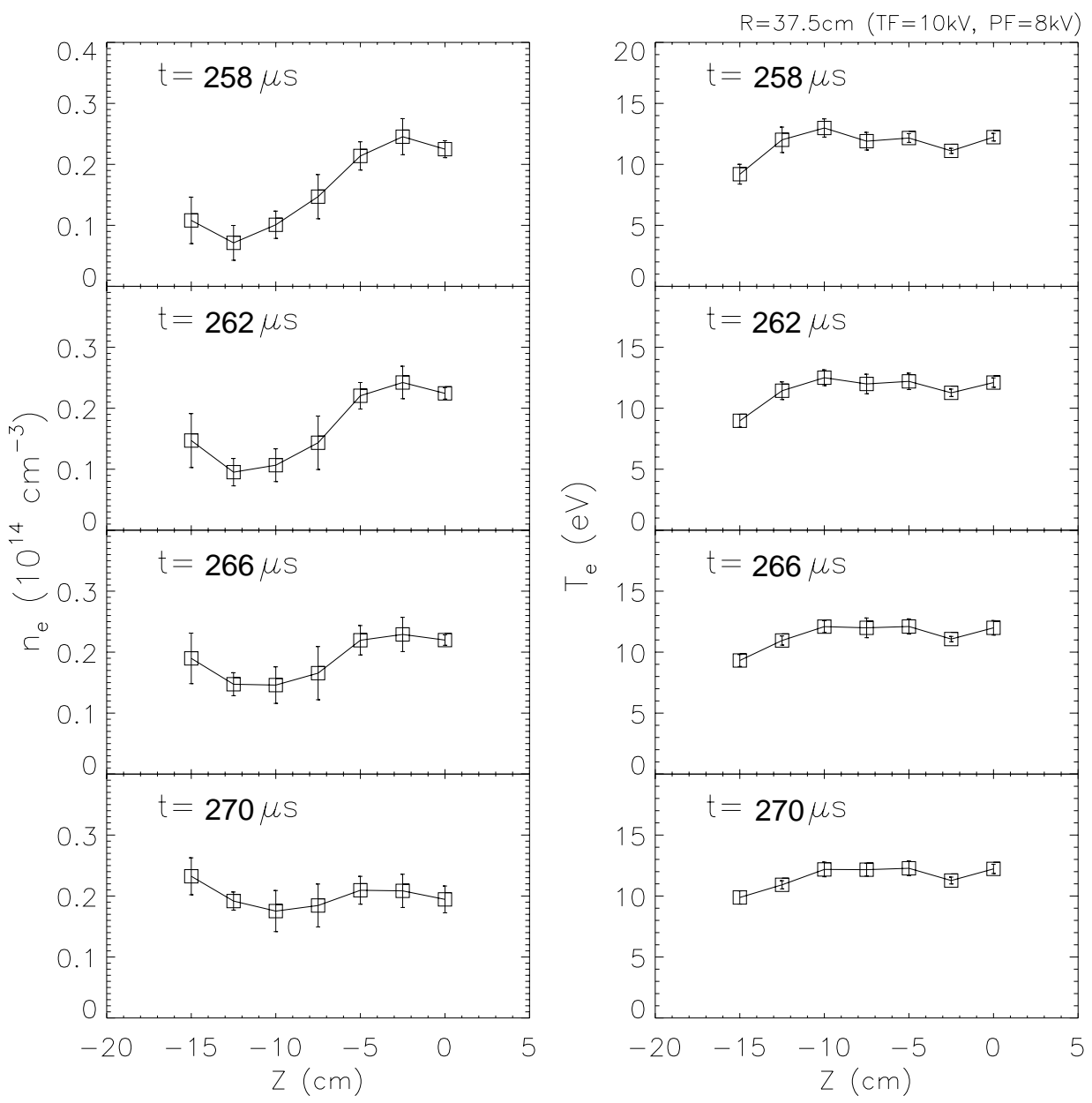

Figure 4.8: Triple Langmuir probe measurements of $n_{\mathrm{e}}$ and $T_{\mathrm{e}}$ as a function of $Z$ and time for null-helicity, hydrogen discharges, showing an example of how downstream pressure (at $Z<-10 \mathrm{~cm}$ ) builds up during reconnection, consistent with the measured sub-Alfvénic downstream ion flows.

The suppression of Alfvénic downstream flows in MRX due to the buildup of high downstream pressure is interesting and is an example of how global boundary conditions might affect the local reconnection dynamics. In pull reconnection, the downstream region consists of the two "private flux" regions surrounding each fluxcore [see Sec. 2.2 and Fig. 2.4(c)]. In the near future, push reconnection experiments 
will be possible. ${ }^{11}$ In push reconnection, the downstream region would be the "public flux" region [see Fig. 2.4(b)] surrounding both flux cores. The volume of the public flux region is larger than the two private flux regions by roughly an order of magnitude. The buildup of downstream pressure would thus be expected to take an order of magnitude longer for push reconnection, pushing it beyond the duration of reconnection. In this case, it is possible that Alfvénic flows might be observed, with interesting consequences for the reconnection rate (which would be expected to increase) and the ion heating (which would be expected to occur also in the downstream region).

\subsubsection{Toroidal flow}

The out-of-plane reconnection electric field is generally considered to be a likely candidate for particle acceleration to very high energies, especially for highly collisionless events such as solar flares [Somov and KosugI, 1997]. In MRX with an $E_{\theta} \sim 150 \mathrm{~V} / \mathrm{m}$, acceleration via this mechanism is marginal even if particles are allowed to free-stream unimpeded by magnetic fields (it would take more than one classical collision time, $5 \mu$ s for ions and $36 \mathrm{~ns}$ for electrons, for either species to reach a $15 \mathrm{eV}$ thermal speed). In reality, the ions are magnetized at the edge of the layer and follow serpentine orbits throughout most of the layer [SPEISER, 1965] and thus cannot accelerate freely, while the electrons are magnetized down to the $\rho_{\mathrm{e}}$ scale (an unresolvable $0.4 \mathrm{~mm}$ in MRX). From these considerations alone, it may be possible to rule out toroidal ion acceleration as a mechanism for the observed ion heating. Experimental measurements indeed support this hypothesis. Furthermore, the nature of the toroidal flow, e.g. which species contributes more to the current, may hold important clues for understanding the equilibrium properties of the current sheet, as discussed in Sec. 3.7.3. The Harris equilibrium [HARRIS, 1962; YAMADA et al., 2000] implies a diamagnetic current, but this is not observed in the present measurements.

Since the IDSP is physically located in the perfect position to measure toroidal flow, the measurements reported here are based on Doppler shifts of the He II $4686 \AA$ ion line. Mach probe measurements of $V_{\mathrm{i} \theta}$ are also available and indicate

\footnotetext{
${ }^{11}$ Due to the addition of a third capacitor bank, which will allow the push phase to be extended beyond the plasma formation stage.
} 
relatively good agreement (see Appendix A). Thus, these are direct measurements of the majority ion Doppler shift. The IDSP is scanned over several radial positions. At each radial position, 5-10 shots are taken with the sightline at a $45^{\circ}$ angle to the $+\theta$ direction, and then this is repeated with the sightline at a $45^{\circ}$ angle to the $-\theta$ direction. The averaged difference (accounting for the angle of course) gives the relative Doppler shift, which translates to an absolute toroidal flow velocity. CCD gate time is $t=250 \rightarrow 270 \mu \mathrm{s}$. It is important to recognize that these measurements have limitations due to averaging effects of the $5 \mathrm{~cm}$ light collection volume and the $10 \mu \mathrm{s}$ CCD gate time and thus may be underestimating the true local $V_{\mathrm{i} \theta}$.

Shown in Fig. 4.9 is the radial profile of toroidal flow speed $V_{\mathrm{i} \theta}$ for null-helicity

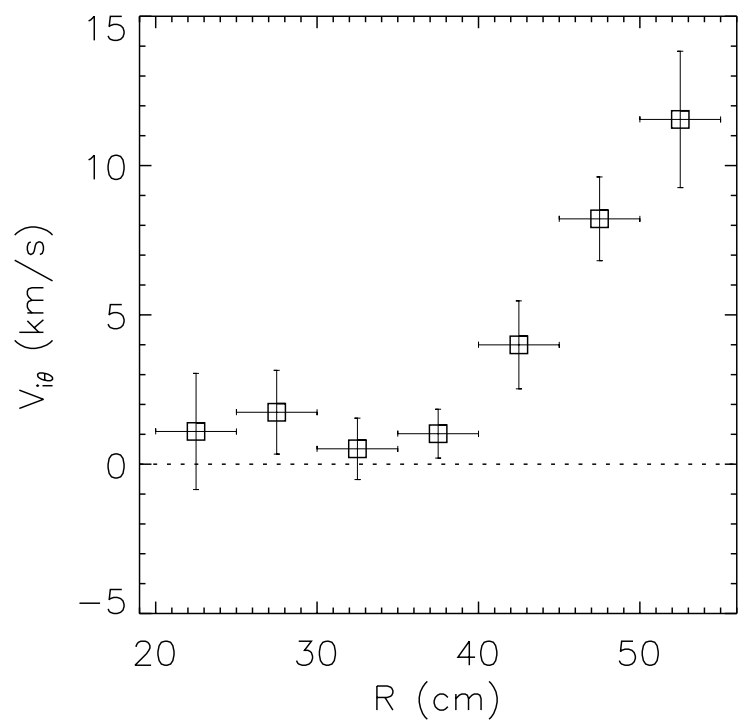

Figure 4.9: IDSP measurement of toroidal ion flow $V_{\mathrm{i} \theta}$ at $Z=0 \mathrm{~cm}$ and $t=250 \rightarrow$ $270 \mu$ s for null-helicity reconnection (shots 10981-11097). Ion flow is energetically insignificant inside the reconnection layer $(R \approx 37 \mathrm{~cm})$ but rises on the outside, where there is no toroidal current, to nearly the ion thermal velocity.

reconnection. Vertical error bars represent shot-to-shot variation and horizontal error bars represent the spatial extent of the IDSP. The profile shape is somewhat unexpected, but it has been verified many times by different measurements (Mach probe and chord-averaged spectroscopy). Since there is very little current beyond $R=40 \mathrm{~cm}$, these measurements imply that the whole plasma (not just ions) is rotating on the outside. Most importantly, note that the magnitude of $V_{\mathrm{i} \theta}$ in the 
layer $(R \approx 35 \rightarrow 40 \mathrm{~cm})$ is only one-tenth the ion thermal speed $(\approx 20 \mathrm{~km} / \mathrm{s}$ for $15 \mathrm{eV}$ helium ions) and cannot account for the observed ion heating via viscosity, which is estimated later in Sec. 4.3.6. ${ }^{12}$ The co-helicity case is quite different, as shown in Fig. 4.10. The flow profile is symmetric and peaked slightly outside the current sheet. The peak value of $V_{\mathrm{i} \theta}$ is higher than for the null-helicity case. However, $V_{\mathrm{i} \theta}$ in the layer, only a few $\mathrm{km} / \mathrm{s}$ at most $(<1 \mathrm{eV})$, is also insufficient to account for the smaller observed ion heating for co-helicity reconnection.

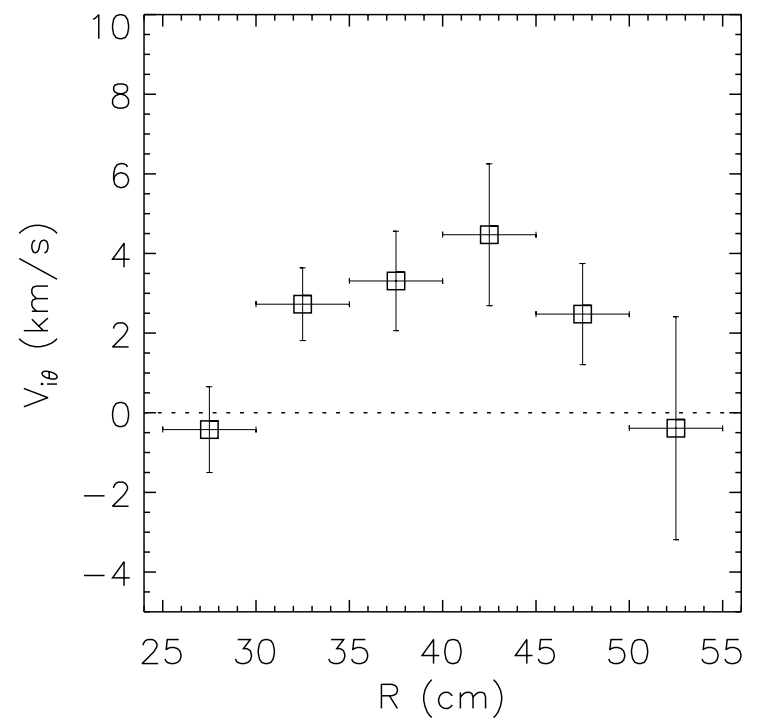

Figure 4.10: IDSP measurement of toroidal ion flow $V_{\mathrm{i} \theta}$ at $Z=0 \mathrm{~cm}$ and $t=250 \rightarrow$ $270 \mu$ s for co-helicity reconnection (shots 11343-11428), showing a more symmetric profile.

The important conclusion of the toroidal flow measurements is that the observed ion heating is not due to acceleration of energetic toroidal ion flows in the layer. In the reconnection layer, the instantaneous energy density of these flows $\left(\rho V_{\mathrm{i} \theta}^{2} / 2 \lesssim\right.$ $1.5 \mathrm{~J} / \mathrm{m}^{3}$ ) is two orders of magnitude smaller than the observed ion thermal energy density $\left(\approx 120 \mathrm{~J} / \mathrm{m}^{3}\right.$, as given in the previous section). Regarding the toroidal

\footnotetext{
${ }^{12}$ It may be possible, however, for ions to be heated outside the layer (beyond $R \approx 45 \mathrm{~cm}$ ) due to scattering of rotating ions off neutrals [ANDEREGG et al., 1986], depending on the degree of ionization. However, the density far outside the layer is down by an order of magnitude $\left(n_{\mathrm{e}} \sim 5 \times 10^{12} \mathrm{~cm}^{-3}\right)$ compared to the center of the reconnection layer, and thus there is not much total energy content. Is not known at present if and how this plasma rotation is related to the reconnection process.
} 
current, the magnitude of the measured $V_{\mathrm{i} \theta}$ implies that the current cannot be diamagnetic, which would predict an ion drift speed of $j_{\theta} / 2 n e \sim 30 \mathrm{~km} / \mathrm{s}$ (using $j_{\theta}=0.5 \mathrm{MA} / \mathrm{m}^{2}$ and $n_{\mathrm{e}}=5 \times 10^{13} \mathrm{~cm}^{-3}$ ). Below, speculation regarding the contradiction is given.

\section{Speculations regarding toroidal ion flow}

The value of peak toroidal current density in the layer, $j_{\theta} \approx 0.5 \mathrm{MA} / \mathrm{m}^{2}$, derived from $B_{\mathrm{Z}}$ measurements implies a relative drift between ions and electrons of $V_{\mathrm{D}} \equiv V_{\mathrm{i} \theta}-V_{\mathrm{e} \theta}=j_{\theta} / n e \approx 62 \mathrm{~km} / \mathrm{s}$ (using $n_{\mathrm{e}}=5 \times 10^{13} \mathrm{~cm}^{-3}$ ). The toroidal flow measurements are not consistent with this. The deviation from the expected diamagnetic picture as well as the difference in $V_{\mathrm{i} \theta}$ between null-helicity and co-helicity reconnection might be explained by the different radial electric fields $E_{\mathrm{R}}$ present in the two cases. Langmuir probe floating potential $V_{\mathrm{f}}$ measurements are shown in Figs. 4.11 and 4.12 for null-helicity and co-helicity, respectively. In the null-helicity case, $V_{\mathrm{f}}$ decreases monotonically with $R$, yielding very crudely ${ }^{13} E_{\mathrm{R}} \sim 1000 \mathrm{~V} / \mathrm{m}$. The resulting toroidal $E_{\mathrm{R}} \times B_{\mathrm{Z}}$ drift $[\sim(1000 \mathrm{~V} / \mathrm{m}) /(0.03 \mathrm{~T})=33 \mathrm{~km} / \mathrm{s}]$ is the same order of magnitude as the strong ion rotation outside the layer, as seen in the data of Fig. 4.9, and can cancel the contribution of ion diamagnetic drift in the layer. This possibly explains why the diamagnetic drift speed is not observed in the $V_{\mathrm{i} \theta}$ measurements. In contrast, the $V_{\mathrm{f}}$ profile for co-helicity reconnection is essentially flat, meaning there is no macroscopic radial electric field. In this case, $V_{\mathrm{i} \theta}(R)$ is fairly symmetric and limited in magnitude. In the co-helicity case, the current would not be expected to be diamagnetic since there is a strong flow component parallel to the toroidal field. It is unclear what ion drift speed ought to be expected in the co-helicity case. Clearly, further investigations are necessary in order to understand the dynamics which determine the observed characteristics of toroidal ion flow and probe potential $V_{\mathrm{f}} .{ }^{14}$ Obvious first steps are to obtain floating potential measurements beyond $R=44 \mathrm{~cm}$ and to use a Langmuir probe array to obtain $V_{\mathrm{f}}$ measurements simultaneously at several radial positions, thus eliminating

\footnotetext{
${ }^{13}$ The floating probe potential $V_{\mathrm{f}}$ is related to the plasma potential usually through a function of $T_{\mathrm{e}}$. Since the $T_{\mathrm{e}}$ profile is fairly broad, taking it as constant is sufficient for this crude estimate of $E_{\mathrm{R}}$.

${ }^{14}$ As mentioned in Sec. 3.7.3, the observed potentials are also a significant deviation from the Harris theory.
} 
the effects of shot-to-shot variation.

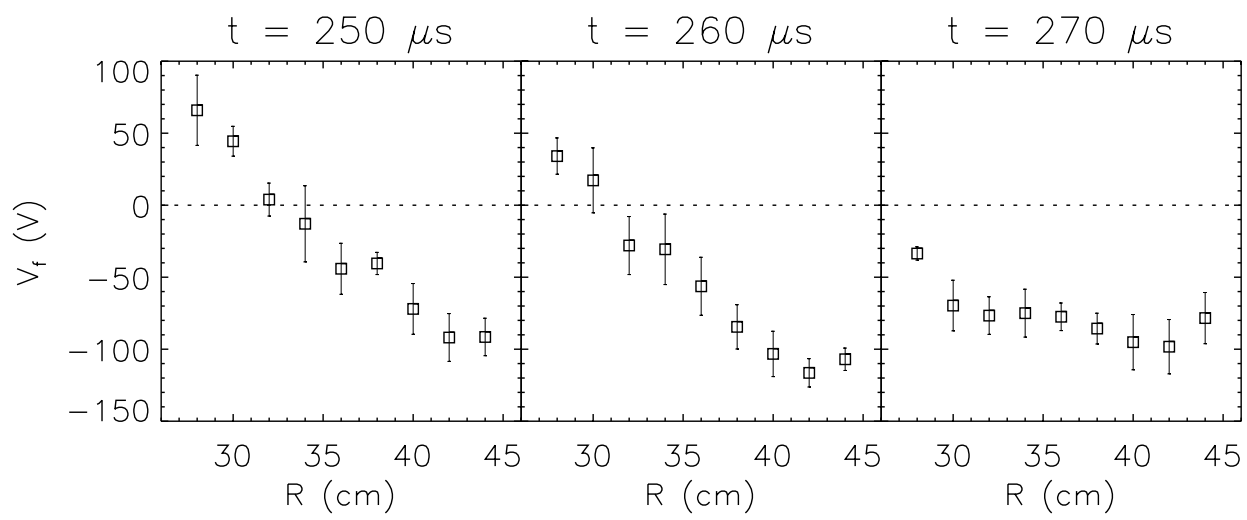

Figure 4.11: Radial profile of Langmuir probe floating potential (with respect to machine ground) at three times during null-helicity reconnection (shots 1101011133).

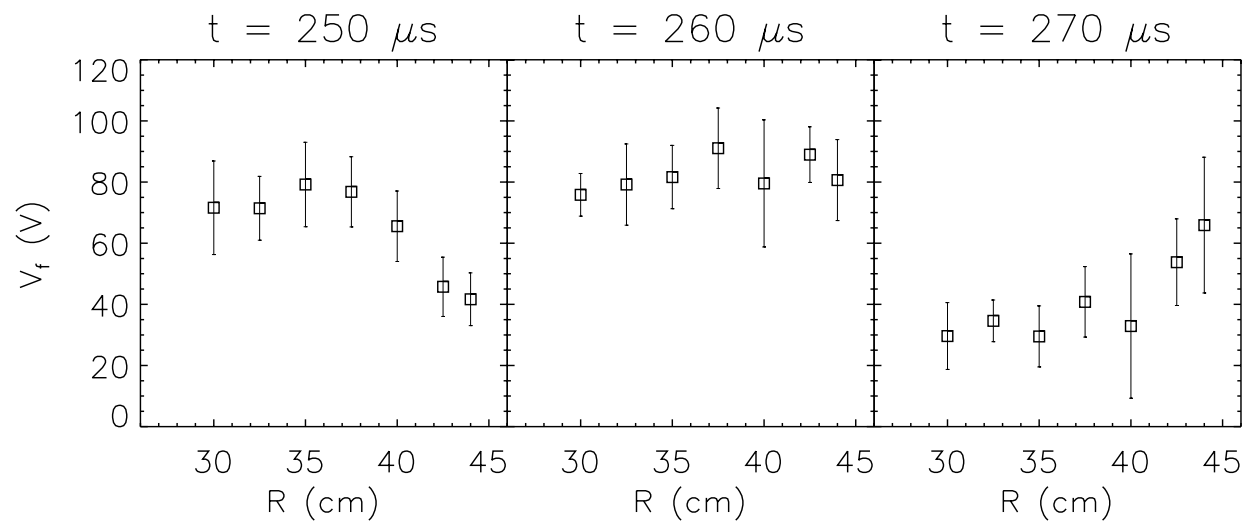

Figure 4.12: Radial profile of Langmuir probe floating potential (with respect to machine ground) at three times during co-helicity reconnection (shots 11386-11424).

\subsection{Ion energy balance during reconnection}

In this section, an energy balance for ions during null-helicity reconnection is considered in detail. The goal is to determine the energy gained by ions during the reconnection process and how much of that energy is converted non-classically. The 
energy balance equation used here is based on Eq. (1.23) of [BRAGINSKII, 1965]:

$$
\frac{3}{2} \frac{\partial\left(n T_{\mathrm{i}}\right)}{\partial t}+n T_{\mathrm{i}} \boldsymbol{\nabla} \cdot \mathbf{V}+\boldsymbol{\nabla} \cdot\left(\frac{3}{2} n T_{\mathrm{i}} \mathbf{V}\right)+\boldsymbol{\nabla} \cdot \mathbf{q}-Q_{\mathrm{i}-\mathrm{n}}=\underbrace{Q_{\text {vis }}+Q_{\mathrm{i}-\mathrm{e}}}_{Q_{\text {classical }}}+Q_{\text {noncl }},
$$

where the terms on the LHS are (from left to right) rise in ion thermal energy density, a compression term including work done by pressure, convective heat loss, conductive heat loss, and energy lost to neutrals. The terms on the RHS are sources of energy for the ions, including (from left to right) viscous heating, heating due to electron-ion collisions, and any non-classical ion heating mechanisms (e.g. due to wave-particle interactions). The first two terms on the RHS represent classical heating mechanisms. The total ion heating is some fraction of the reconnected field energy, which is known from $E_{\theta} j_{\theta}$ derived from magnetic probe measurements. Each term of Eq. (4.1) will be estimated based on experimental data wherever possible. Note that $Q_{\mathrm{i}-\mathrm{e}}$ can be neglected immediately since the characteristic classical ionelectron energy equilibration time is more than $400 \mu \mathrm{s}$ and thus irrelevant on the time scale of the reconnection process $(30 \mu \mathrm{s})$. Incidentally, note that the kinetic energy of flows, i.e. $\partial\left(\rho V_{\mathrm{i}}^{2} / 2\right) / \partial t$, does not appear explicitly in Eq. (4.1), which is only one possible way to express the ion energy balance. In this case, it has been eliminated by use of the continuity and momentum equations [BRAGINSKII, 1965].

The results, to be presented in detail, show that a substantial fraction (more than half) of the reconnected field energy is converted to ion energy, and mostly due to non-classical mechanisms. These results differ from the TS-3 results [ONO et al., 1996; ONO et al., 1997], in which ion heating was attributed predominantly to viscous damping of Alfvénic ion flows, and from classical MHD reconnection models, in which ions are heated both by viscosity and by energy exchange with Ohmically heated electrons.

In discussing an energy budget, a finite volume and time duration must be defined. Here, the energy budget is considered for a given volume $V=5.9 \times 10^{-3} \mathrm{~m}^{3}$, which is the area monitored by the $\operatorname{IDSP}(R=35 \rightarrow 40 \mathrm{~cm}$ and $Z=-2.5 \rightarrow$ $2.5 \mathrm{~cm}$ ) revolved around the axis of symmetry. The volume $V$ is essentially a toroid coinciding with the center of the toroidal current sheet, with the area monitored by the IDSP as its toroidal cross section. The time duration $\Delta t$ is defined as 
$t_{1}=245 \rightarrow t_{2}=265 \mu \mathrm{s}$, which is during the pull reconnection phase. The data are from a set of null-helicity discharges with the same parameters ${ }^{15}$ as the ones in Figs. 4.1 and 4.6. Note that all the calculations in this section invoke axisymmetry, as discussed in Sec. 3.1, since measurements are only known at one toroidal position.

\subsubsection{Reconnected field energy}

Before examining the terms in Eq. (4.1), the energy released due to reconnection will be calculated. Section 3.6 discusses how dissipated magnetic field energy can be calculated according to

$$
W_{\mathrm{rec}}=\int_{t_{1}}^{t_{2}} \int_{V} \mathbf{E} \cdot \mathbf{j} d^{3} V d t \approx \int_{t_{1}}^{t_{2}} \int_{V} E_{\theta} j_{\theta} d^{3} V d t
$$

which represents the total energy due to reconnection available to heat ions (and electrons). The toroidal electric field $E_{\theta}$ and current density $j_{\theta}$ as a function of space and time are shown in Figs. 4.13 and 4.14, respectively.

Note that $E_{\theta}$ is relatively uniform in space, while $j_{\theta}$ is peaked near $R=37 \mathrm{~cm}$. Both quantities increase in time as pull reconnection proceeds and then reach a short flat-top around $t=260 \mu$ s before starting to decrease. Energy dissipation is clearly strongest in the reconnection layer where $j_{\theta}$ is concentrated. Using the $E_{\theta}$ and $j_{\theta}$ data in Eq. (4.2) gives $W_{\text {rec }} \approx 4.8 \pm 0.7 \mathrm{~J}^{16}$ It should be noted that the volume $V$, is only a fraction of the total reconnection region, and that the total energy dissipated in the entire reconnection region during $\Delta t$ is estimated to be on the order of $30 \mathrm{~J}$.

\footnotetext{
${ }^{15} \mathrm{TF} / \mathrm{PF}$ bank voltages $=13 / 11 \mathrm{kV}$, initial gas pressure $=6 \mathrm{mT}$, helium discharges

${ }^{16}$ The given error is based on the standard deviation of the mean, see e.g. [BEVINGTON and RoBInson, 1992], of $E_{\theta}$ and $j_{\theta}$ since these values used are taken from an average of approximately 30 shots. All the error values reported for the energy budget analysis henceforth in this chapter are based on the standard deviation of the mean. In reporting raw measurements in this dissertation (such as in figures), the error bars represent the standard deviation of an ensemble of shots, not the standard deviation of the mean. This gives the reader more complete information regarding the shot-to-shot variation. However, because the calculations in this section are based on averaged values, the standard deviation of the mean is the more meaningful error estimate.
} 


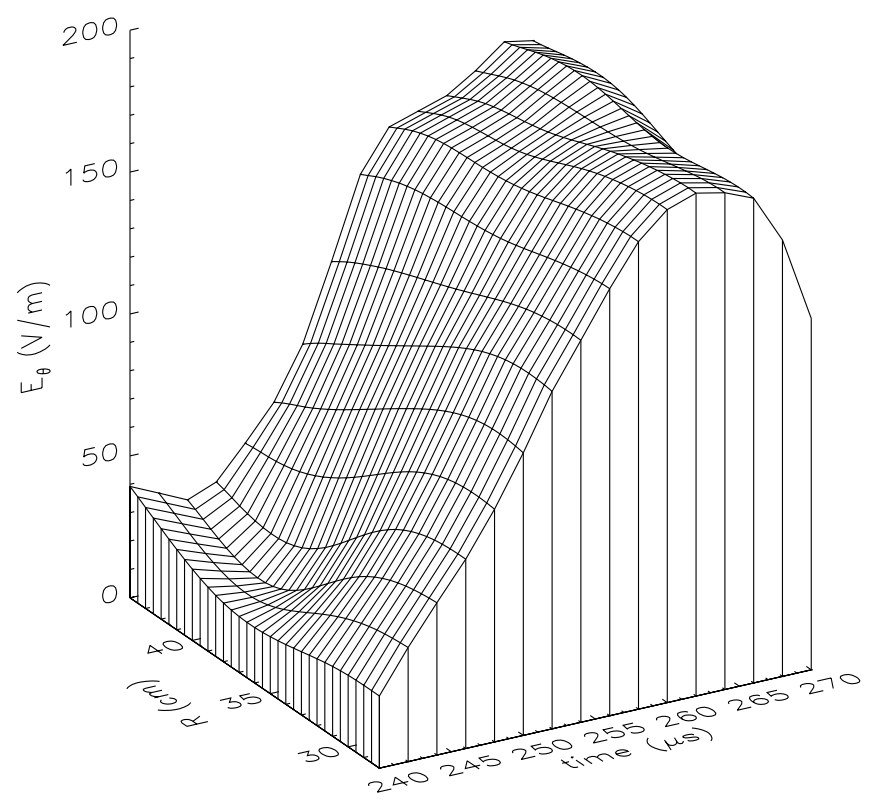

Figure 4.13: Surface plot of null-helicity $E_{\theta}$ as a function of radius and time, averaged over $Z=-2.5 \rightarrow 2.5 \mathrm{~cm}$ (from 90-channel probe measurements, averaged over shots 12233-12266).

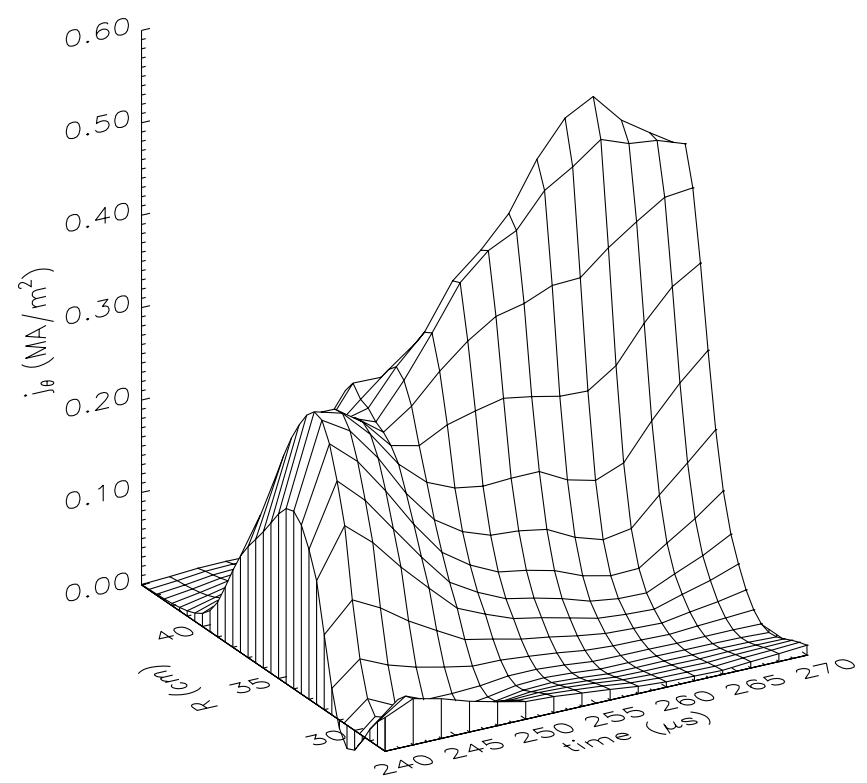

Figure 4.14: Surface plot of null-helicity $j_{\theta}$, which is peaked in the reconnection layer, as a function of radius and time at $Z=0 \mathrm{~cm}$ (from high-resolution 1-D probe measurements, averaged over shots 12233-12266). 


\subsubsection{Increase in ion thermal energy}

The increase in ion thermal energy, the first term on the LHS of Eq. (4.1), in volume $V$ during reconnection is calculated as follows,

$$
\Delta W_{\mathrm{th}, \mathrm{i}} \equiv \frac{3}{2}\left[\bar{n}\left(t_{2}\right) T_{\mathrm{i}}\left(t_{2}\right)-\bar{n}\left(t_{1}\right) T_{\mathrm{i}}\left(t_{1}\right)\right] V \approx 0.5 \pm 0.2 \mathrm{~J},
$$

where $\bar{n}\left(t_{1}\right) \approx \bar{n}\left(t_{2}\right) \approx 5 \pm 1 \times 10^{13} \mathrm{~cm}^{-3}$ is the density averaged over area $A$, and $T\left(t_{1}\right) \approx 6 \pm 1 \mathrm{eV}$ and $T\left(t_{2}\right) \approx 13 \pm 2 \mathrm{eV}$ (see Fig. 4.1). Note that $\Delta W_{\mathrm{th}, \mathrm{i}}$ is only the remnant ion thermal energy in $V$ and does not include ion heat loss during $\Delta t$. The rise in ion thermal energy is due predominantly to a rise in $T_{\mathrm{i}}$, while the density in the layer remains fairly constant during $\Delta t$, as shown in Fig. 4.15.

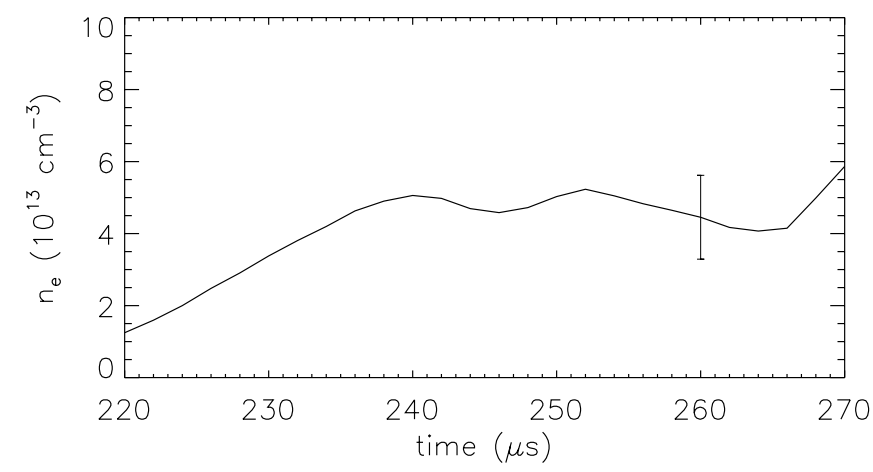

Figure 4.15: Time evolution of $n_{\mathrm{e}}$ measured by triple Langmuir probe at $R=$ $37.5 \mathrm{~cm}$ and $Z=0 \mathrm{~cm}$.

As an aside, an additional set of experiments in which the firing voltage was varied is reported here. A range of $\Delta W_{\text {th,i }}$ and $W_{\text {rec }}$ were obtained in this experiment. Figure 4.16 shows that $\Delta W_{\mathrm{th}, \mathrm{i}}$ scales with $W_{\text {rec }}$, i.e. $T_{\mathrm{i}}$ rises more as more energy is released due to reconnection. Each data point represents the average of 5 plasma shots at a given firing voltage, and error bars (from data point to extreme) represent one standard deviation in the shot-to-shot scatter. This is consistent with the conclusion from Sec. 4.1 that the observed rise in $T_{\mathrm{i}}$ is causally linked to reconnection. 


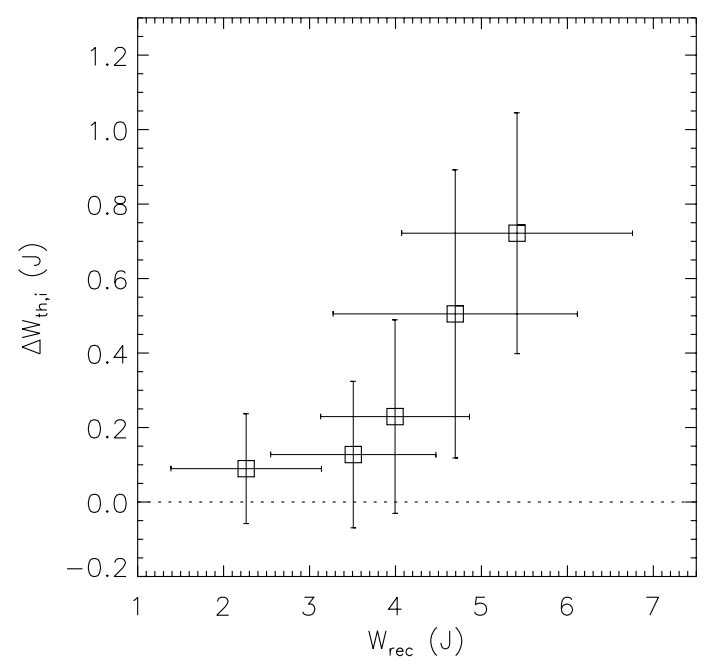

Figure 4.16: Observed increase in ion thermal energy $\Delta W_{\mathrm{th}, \mathrm{i}}$ versus reconnected field energy $W_{\text {rec }}$ for null-helicity discharges with varying firing voltages.

\subsubsection{Compression}

The second term on the LHS of Eq. (4.1) is a compression term including the work done by pressure forces. It can be estimated approximately as $\left\langle n T_{\mathrm{i}}\left(\partial V_{\mathrm{R}} / \partial R+\right.\right.$ $\left.\left.\partial V_{\mathrm{Z}} / \partial Z\right)\right\rangle V \Delta t,{ }^{17}$ where the brackets indicate a spatial average over $V$ and temporal average over $\Delta t$. Using values of $n \approx 5 \pm 1 \times 10^{13} \mathrm{~cm}^{-3}, T_{\mathrm{i}} \approx 10 \pm 1 \mathrm{eV}, \partial V_{\mathrm{R}} / \partial R \approx$ $-(4.8 \mathrm{~km} / \mathrm{s}) /(0.05 \mathrm{~m})=-9.6 \pm 1 \times 10^{4} \mathrm{~s}^{-1}$, and $\partial V_{\mathrm{Z}} / \partial Z \approx(8 \mathrm{~km} / \mathrm{s}) /(0.1 \mathrm{~m})=$ $8 \pm 2 \times 10^{4} \mathrm{~s}^{-1}$, the compression term is $W_{\text {compression }} \approx-0.16 \pm 0.31 \mathrm{~J}$. The negative value means that there is some ion heating in volume $V$ due to compression. The large relative error arises due to the subtraction of the velocity gradient terms, which results in a small number with large relative error. It will be shown that this compression term is small compared to the ion loss terms, and thus the rough estimate adopted above is justified.

\subsubsection{Ion energy losses}

Since volume $V$ is an open system, a complete energy budget must consider ion energy losses due to convective and conductive heat loss. The energy lost must

\footnotetext{
${ }^{17}$ The $V_{\mathrm{R}} / R$ term of $\boldsymbol{\nabla} \cdot \mathbf{V}$ averages to nearly zero over $V$ and is neglected.
} 
be accounted for since their original source was the dissipated magnetic energy. The heat conduction estimates in this sub-section are based on classical transport. Therefore, they represent a lower bound on ion energy loss since the transport is almost certainly not classical. It is important to emphasize that this would only lead to an underestimate of energy conversion to ions. In general, ions may also lose energy to neutrals, and this is discussed.

\section{Convection}

Convective heat loss, the third term on the LHS of Eq. (4.1), out of $V$ during $\Delta t$ can be estimated as the heat convected out of $V$,

$$
W_{\text {out }} \approx\left[\frac{3}{2} n(\text { layer }) T_{\mathrm{i}}(\text { layer })\right] \times(\text { volume of plasma out }),
$$

minus the heat convected into $V$,

$$
W_{\text {in }} \approx\left[\frac{3}{2} n(\text { upstream }) T_{\mathrm{i}}(\text { upstream })\right] \times(\text { volume of plasma in })
$$

during $\Delta t$. The volumes of plasma flowing out of and into $V$ are determined based on the outflow speed $V_{\mathrm{iz}} \approx 3 \pm 0.8 \mathrm{~km} / \mathrm{s}$ and inflow speed $V_{\mathrm{iR}} \approx 3 \pm 0.4 \mathrm{~km} / \mathrm{s}$, respectively. At the boundaries of volume $V$, the two speeds are approximately equal and thus the volumes convected in and out are also equal $\left(1.4 \times 10^{-2} \mathrm{~m}^{3}\right)$. The density in the layer $n$ (layer) is approximately $5 \pm 1 \times 10^{13} \mathrm{~cm}^{-3}$, and the average upstream density is approximately $3 \pm 0.4 \times 10^{13} \mathrm{~cm}^{-3}$. The layer $T_{\mathrm{i}}$ is approximately $12 \pm 0.9 \mathrm{eV}$ and the average upstream $T_{\mathrm{i}} \approx 10 \pm 1.3 \mathrm{eV} .{ }^{18}$ These numbers combine to yield a total ion energy loss due to convection out of volume $V$ during time $\Delta t$ of $W_{\text {convection }}=W_{\text {out }}-W_{\text {in }} \approx 1.0 \pm 0.7 \mathrm{~J}$.

\footnotetext{
${ }^{18}$ The measured radial $T_{\mathrm{i}}$ profiles are broad. However, it must be mentioned that the minimum CCD gate-opening time of $10 \mu \mathrm{s}$ is likely longer than the time it takes for any sharp gradients in $T_{\mathrm{i}}$ to flatten, and this likely leads to an underestimate of $W_{\text {convection }}$ due to an underestimate of $\nabla T_{\mathrm{i}}$.
} 


\section{Conduction}

Ion heat loss due to thermal conduction is estimated using classical transport. This estimate likely represents a lower bound because (1) $T_{\mathrm{i}}$ gradients may be underestimated due to the time resolution of the measurements, and (2) enhanced non-classical transport mechanisms are unknown but almost certainly exist. Ion energy loss due to classical conduction can be written as [BRAGINSKII, 1965]

$$
\boldsymbol{\nabla} \cdot \mathbf{q}=-\nabla_{\perp} \cdot\left(\kappa_{\perp} \nabla_{\perp} T_{\mathrm{i}}\right)-\nabla_{\|}\left(\kappa_{\|} \boldsymbol{\nabla}_{\|} T_{\mathrm{i}}\right)+\boldsymbol{\nabla} \cdot\left[\frac{5 c n T_{\mathrm{i}}}{2 e B}\left(\mathbf{B} / B \times \nabla T_{\mathrm{i}}\right)\right]
$$

where the last term on the RHS vanishes since $\left(\mathbf{B} / B \times \nabla T_{\mathrm{i}}\right)$ has only a $\theta$ component and $\partial / \partial \theta=0$, and $\kappa_{\perp}$ and $\kappa_{\|}$are the perpendicular and parallel ion thermal conductivities, respectively [BRAGINSKII, 1965]:

$$
\begin{aligned}
\kappa_{\perp} & =\frac{2 n T_{\mathrm{i}}}{m_{\mathrm{i}} \omega_{\mathrm{ci}}^{2} \tau_{\mathrm{i}}} \approx 2.8 \pm 0.6 \times 10^{22} \frac{1}{\mathrm{~m} \cdot \mathrm{s}} \\
\kappa_{\|} & =\frac{3.9 n T_{\mathrm{i}} \tau_{\mathrm{i}}}{m_{\mathrm{i}}} \approx 11.0 \pm 2.2 \times 10^{22} \frac{1}{\mathrm{~m} \cdot \mathrm{s}}
\end{aligned}
$$

Average values during $\Delta t$ have been used: $n=5 \pm 1 \times 10^{13} \mathrm{~cm}^{-3}, T_{\mathrm{i}}=10 \pm 1 \mathrm{eV}$, and $B=250 \mathrm{G}$. The total surface area of $V$ is $0.48 \mathrm{~m}^{2}$, and it is equally divided between being intercepted by $B_{\mathrm{Z}}$ in the perpendicular and parallel directions. Using these values and $\nabla_{\perp} T_{\mathrm{i}} \approx(2 \pm 1 \mathrm{eV}) /(5 \mathrm{~cm})$ and $\nabla_{\|} T_{\mathrm{i}} \approx(1 \pm 0.5 \mathrm{eV}) /(10 \mathrm{~cm}),{ }^{19}$ the ion heat loss due to classical transport is estimated to be $W_{\text {conduction }} \approx 1.7 \pm 0.7 \mathrm{~J}$.

\section{Collisions with neutrals}

Ion-neutral collisions can be another energy loss channel for heated ions. In MRX regimes, charge exchange is expected to be the dominant ion-neutral interaction. By any reasonable estimate, the plasma inside the current sheet with $T_{\mathrm{e}}>15 \mathrm{eV}$ should be better than $99 \%$ ionized. The cross section for $\mathrm{He}^{-} \mathrm{He}^{+}$charge exchange for $10 \mathrm{eV}$ ions is $\langle\sigma v\rangle \approx 4 \times 10^{-9} \mathrm{~cm}^{3} / \mathrm{s}^{20}$ The ion density is approximately

\footnotetext{
${ }^{19}$ The perpendicular gradient is based on IDSP measurements. However, the parallel gradient was not measured, and thus a conservative lower limit had to be assumed.

${ }^{20}$ See e.g. [JANEV et al., 1987].
} 
$5 \times 10^{13} \mathrm{~cm}^{-3}$. A $1 \%$ concentration of neutrals ${ }^{21}$ would result in a charge exchange time of $500 \mu \mathrm{s}$, completely negligible on the reconnection time scale of $30 \mu \mathrm{s}$. It would take more than a $50 \%$ concentration of neutrals in the reconnection layer to bring down the charge exchange time to a more relevant $10 \mu \mathrm{s}$. A $50 \%$ neutral concentration in the presence of $15 \mathrm{eV}$ electrons is highly unlikely. Therefore, ion energy loss to neutrals is neglected. In any case, note that ion-neutral energy loss would only increase the estimate of ion heating due to reconnection.

\subsubsection{Classical viscous heating}

Classical heating per unit volume due to viscosity in the absence of a magnetic field (justified since ions are unmagnetized in the layer) is [BRAGINSKII, 1965]

$$
Q_{\mathrm{vis}}=\eta_{0} W_{\alpha \beta} \frac{\partial V_{\alpha}}{\partial x_{\beta}}
$$

where $\eta_{0}=0.96 n T_{\mathrm{i}} \tau_{\mathrm{i}} \approx 1.8 \times 10^{-4} \mathrm{~J} \cdot \mathrm{s} / \mathrm{m}^{3}$ (using $n=5 \times 10^{13} \mathrm{~cm}^{-3}$ and $\left.T_{\mathrm{i}}=10 \mathrm{eV}\right)$, and the rate-of-strain tensor is

$$
W_{\alpha \beta}=\frac{\partial V_{\alpha}}{\partial x_{\beta}}+\frac{\partial V_{\beta}}{\partial x_{\alpha}}-\frac{2}{3} \delta_{\alpha \beta} \boldsymbol{\nabla} \cdot \mathbf{V}
$$

The non-zero velocity gradients averaged over volume $V$ are ${ }^{22}$

$$
\begin{aligned}
\frac{\partial V_{\mathrm{R}}}{\partial R} & \approx-\frac{4.8 \mathrm{~km} / \mathrm{s}}{0.05 \mathrm{~m}}=-9.6 \times 10^{4} \mathrm{~s}^{-1} \\
\frac{\partial V_{\mathrm{Z}}}{\partial Z} & \approx \frac{8 \mathrm{~km} / \mathrm{s}}{0.1 \mathrm{~m}}=8.0 \times 10^{4} \mathrm{~s}^{-1} \\
\left|\frac{\partial V_{\mathrm{Z}}}{\partial R}\right| & \lesssim \frac{3 \mathrm{~km} / \mathrm{s}}{0.05 \mathrm{~m}}=6.0 \times 10^{4} \mathrm{~s}^{-1} \\
\frac{\partial V_{\theta}}{\partial R} & \lesssim \frac{2 \mathrm{~km} / \mathrm{s}}{0.05 \mathrm{~m}}=4.0 \times 10^{4} \mathrm{~s}^{-1},
\end{aligned}
$$

\footnotetext{
${ }^{21}$ Both the Baratron gauge reading of $6 \mathrm{mT} \rightarrow 2.1 \times 10^{14} \mathrm{~cm}^{-3}$ and Langmuir probe density measurement might be expected to have factor of two uncertainties. In this case, the Langmuir probe density reading seems the more accurate one because it gives a value of $n_{\mathrm{e}}$ which satisfies pressure balance across the reconnection layer, i.e. $n\left(T_{\mathrm{i}}+T_{\mathrm{e}}\right)$ inside the layer balances with $B^{2} / 2 \mu_{0}$ outside the layer.

${ }^{22}$ The $Z$ and $\theta$ flows are based on Mach probe and IDSP measurements, and the $R$ flows are based on taking the $E_{\theta} B_{\mathrm{Z}}$ inflow velocity outside the layer and dividing by the scale length of the layer.
} 
and therefore the classical viscous heating per unit volume is

$Q_{\mathrm{vis}} \approx \eta_{0}\left(W_{\mathrm{RR}} \frac{\partial V_{\mathrm{R}}}{\partial R}+W_{\mathrm{ZZ}} \frac{\partial V_{\mathrm{Z}}}{\partial Z}+W_{\mathrm{ZR}} \frac{\partial V_{\mathrm{Z}}}{\partial R}+W_{\theta \mathrm{R}} \frac{\partial V_{\theta}}{\partial R}\right) \approx 6.5 \pm 2.2 \times 10^{6} \mathrm{~J} / \mathrm{s} \cdot \mathrm{m}^{3}$

Using these numbers, the maximum ion energy gained via viscous damping of ion flows is $W_{\text {vis }} \approx Q_{\text {vis }} V \Delta t=0.8 \pm 0.3 \mathrm{~J}$.

\subsubsection{Non-classical ion heating}

The important terms of Eq. (4.1) are summarized in Table 4.1, which gives a quantitative description of energy conversion to ions based on experimental data. The

\begin{tabular}{||l|l||}
\hline process & energy $(\mathrm{J})$ \\
\hline$W_{\text {rec }}$ & $4.8 \pm 0.7$ \\
\hline total $W_{\text {ions }}$ & $3.1 \pm 1.0$ \\
\hline$\Delta W_{\text {th,i }}$ & $0.5 \pm 0.2$ \\
\hline$W_{\text {compression }}$ & $-0.14 \pm 0.28$ \\
\hline$W_{\text {convection }}$ & $1.0 \pm 0.7$ \\
\hline$W_{\text {conduction }}$ & $>1.7 \pm 0.7$ \\
\hline$W_{\text {vis }}$ & $0.8 \pm 0.3$ \\
\hline
\end{tabular}

Table 4.1: Ion energy budget between $t=245 \rightarrow 265 \mu$ s in volume $V$, showing that some $65 \%$ of the dissipated magnetic energy was converted to ions.

numbers show that $65 \pm 21 \%$ of the dissipated field energy is converted to ion energy. Now the question is how much of that energy was converted via non-classical mechanisms. As mentioned before, classical heating due to ion-electron collisions is neglected due to the long ion-electron energy partition time. In any case, energy available due to Ohmic heating is insufficient, estimated to be $\eta_{\mathrm{Sp}} j_{\theta}^{2} V \Delta t \approx 0.2 \mathrm{~J}$ (using $\eta_{\mathrm{Sp}}=2 \times 10^{-5} \Omega \mathrm{m}$ and $j_{\theta} \sim 0.3 \mathrm{MA} / \mathrm{m}^{2}$ ). Note that this is only $4 \%$ of the total dissipated magnetic energy. The other classical mechanism is viscous heating by the ion flow, which was estimated in the previous section to be $0.8 \pm 0.3 \mathrm{~J}$. Subtracting $W_{\text {viscosity }}$ from $W_{\text {ions }}$ leaves $2.3 \pm 1.0 \mathrm{~J}$ of energy which must have been 
converted to ion energy via non-classical mechanisms. Thus, $48 \pm 21 \%$ of the dissipated magnetic energy was converted to ions non-classically. ${ }^{23}$

Although the kinetic energy of ion flows does not appear in Eq. (4.1), an estimate will be given here,

$$
W_{\text {flow }} \equiv \int_{V} \frac{1}{2} \rho V_{\mathrm{i}}^{2} d^{3} V \lesssim 0.05 \mathrm{~J}
$$

which is very small [using a density of $5 \times 10^{13} \mathrm{~cm}^{-3}$ and a flow $V_{\mathrm{i}} \approx\left(V_{\mathrm{iR}}^{2}+V_{\mathrm{iZ}}^{2}+\right.$ $\left.V_{\mathrm{i} \theta}^{2}\right)^{1 / 2}<5 \mathrm{~km} / \mathrm{s}$. Thus, in MRX, ion flows are energetically insignificant, about $1 \%$ of $W_{\text {rec }}$. This is in direct contrast with classical MHD models as well as TS-3 experimental results [ONO et al., 1996; ONO et al., 1997], in which bulk flows are accelerated to $V_{\mathrm{A}}$ during reconnection and account for a significant fraction of the energy balance. The energy distribution is illustrated in Fig. 4.17.

In summary of this section, the energy budget of ions was considered carefully. The total energy released due to reconnection was calculated from experimental data and compared to the different components of ion energy, including observable rise in ion thermal energy and ion heat loss due to convection and conduction. The energy budget showed that some $48 \%$ of the dissipated magnetic energy was converted to ions non-classically. This finding on non-classical ion heating during reconnection, based on experimental measurements, has significant implications for reconnection research since the basic mechanisms which are responsible for enhanced reconnection rates and the energy conversion process are still poorly understood.

\subsection{Resistivity enhancement and ion heating}

In the previous sub-section, it was established that ions must have been heated via non-classical dissipation mechanisms. In this sub-section, experimental data is given suggesting a correlation between ion heating and resistivity enhancement. Resistivity enhancement is defined as the ratio of the measured plasma resistivity

\footnotetext{
${ }^{23}$ The remainder of the energy, about $1.7 \mathrm{~J}$, must go to electrons which, due to their fast parallel thermal transport, show no marked temporal change in $T_{\mathrm{e}}(\approx 15 \mathrm{eV})$ during $\Delta t$. Classical Ohmic heating in $V$ during $\Delta t$ accounts for only about $0.2 \mathrm{~J}$ of the $1.7 \mathrm{~J}$ of energy converted to electrons, also suggesting non-classical heating of electrons. However, electron flow energy is not known.
} 


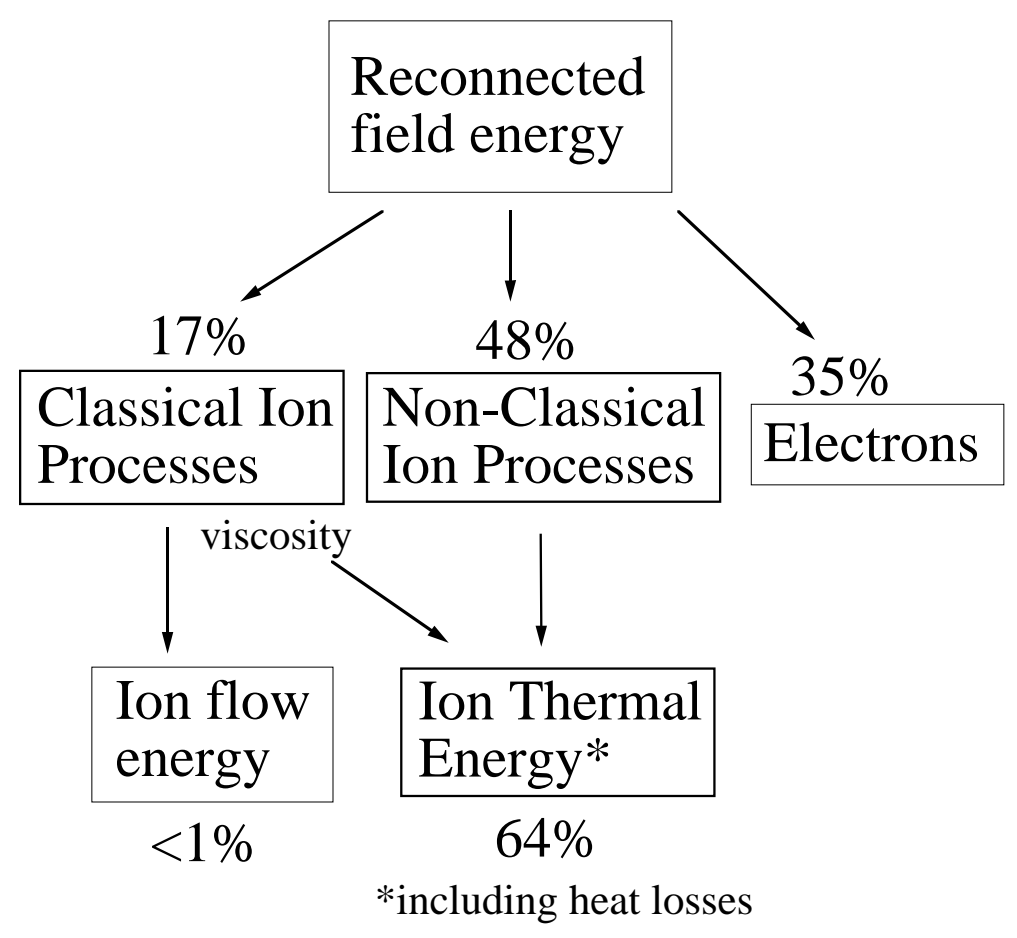

Figure 4.17: Illustration of the field and ion energy budget for null-helicity reconnection. Ions gained $65 \%$ of the dissipated field energy, and $48 \%$ of the dissipated field energy was converted non-classically.

$\eta^{*}$ to the classical Spitzer resistivity $\eta_{\mathrm{Sp}}$, the determinations of which are discussed in Ch. 3. The relative importance of non-classical versus classical dissipation is embodied in the enhancement factor $\eta^{*} / \eta_{\text {Sp }}$, which has been shown to increase as collisionality decreases [Ji et al., 1998] (see Fig. 3.6). One possible explanation for this effect is that as the plasma becomes more collisionless, wave fields can scatter current-carrying particles, increasing $\eta^{*}$ (and possibly also heating ions more efficiently). Generally, enhanced values of resistivity might be expected to be an electron effect since they are expected to carry most of the current. However, in null-helicity reconnection (the case being studied), the current is cross-field, and it is not clear that electrons should be carrying most of the current. In fact, the current may be largely diamagnetic [YAMADA et al., 2000].

By varying the discharge voltage in a set of null-helicity experiments, $\Delta W_{\mathrm{th}, \mathrm{i}} / W_{\text {rec }}$ and $\eta^{*} / \eta_{\text {Sp }}$ are varied, as shown in Fig. 4.18. Increasing the discharge voltage increases reconnection and thus increases ion heating. The scaling of the quantities 


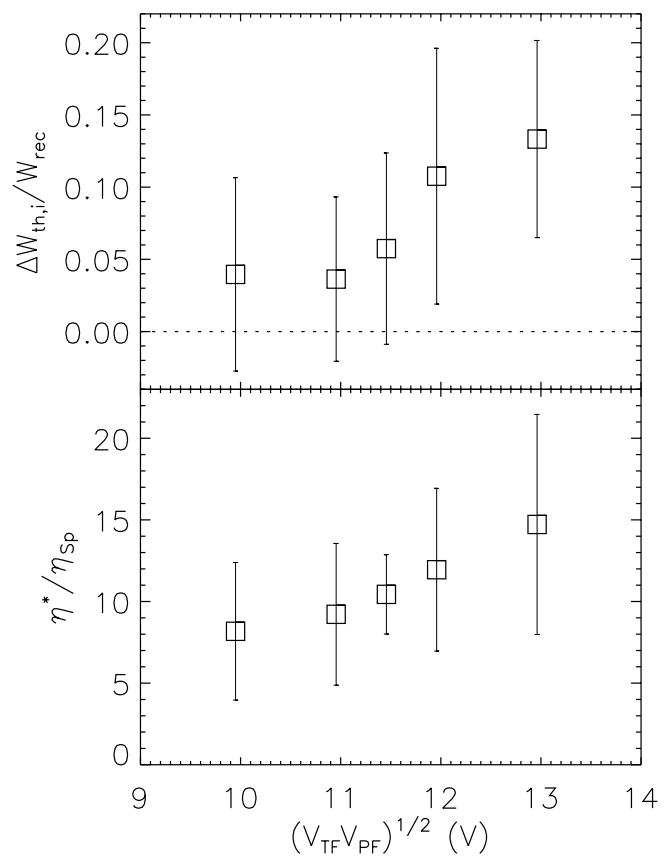

Figure 4.18: (top) Rise in ion thermal energy $\Delta W_{\text {th,i }}$ normalized by reconnected field energy $W_{\text {rec }}$ and (bottom) resistivity enhancement factor $\eta^{*} / \eta_{\mathrm{Sp}}$ versus firing voltage.

with each other can provide insight into the nature of the non-classical ion heating mechanisms. Shown in Fig. 4.19 is $\Delta W_{\text {th,i }} / W_{\text {rec }}$ versus $\eta^{*} / \eta_{\mathrm{Sp}}$. The fraction $\Delta W_{\text {th,i }} / W_{\text {rec }}$ increases from approximately $4 \%$ to $14 \%$ as $\eta^{*} / \eta_{\text {Sp }}$ increases from 8 to 15, as shown in Fig. 4.19. (Note again that $\Delta W_{\mathrm{th}, \mathrm{i}}$ is not the total energy gained by ions but only the remnant ion thermal energy in volume $V$ not including ion heat loss.) Although the error bars are sizable, the trend between the energy converted to ions and the resistivity enhancement is clear. The subtle, and perhaps unexpected, hint provided by Fig. 4.19 is that the (non-classical) mechanism determining the enhanced reconnection rate (and hence increased reconnected field energy) is also responsible for channeling the reconnected field energy to the ions. The non-classical dissipation mechanism in MRX should exhibit this trait. 


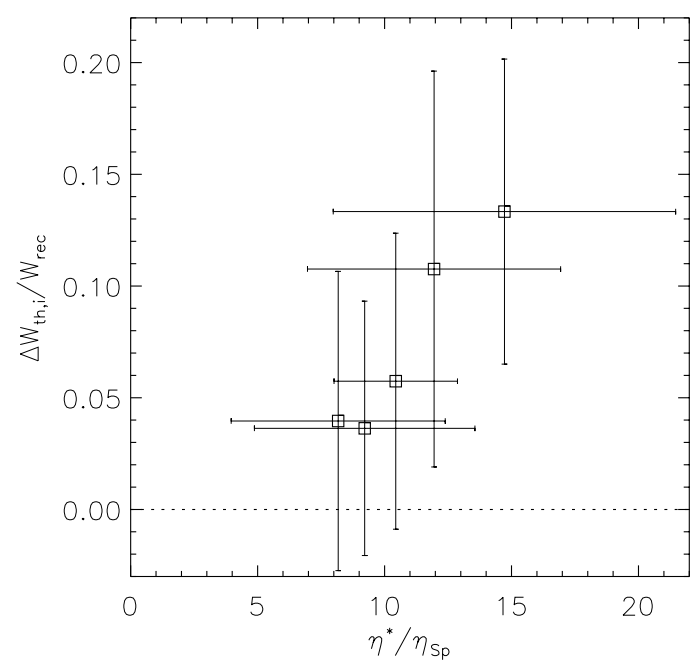

Figure 4.19: Increase in ion thermal energy normalized by reconnected field energy for varying resistivity enhancement factors, showing a correlation between the two.

\subsection{Possible ion heating mechanisms}

The experimental data have indicated the existence of non-classical ion heating, non-classical resistivity, and possibly a correlation between the two. The obvious next step experimentally is to investigate the frequency spectrum of turbulent fluctuations with the possibility of identifying relevant modes which can explain the enhanced resistivity and possibly also the ion heating. ${ }^{24}$ Theoretically, much effort has been devoted to addressing these issues (mostly the resistivity aspect) and a full review is beyond the scope of this work. ${ }^{25}$ Non-classical current sheet dissipation mechanisms can be divided into two classes: wave-particle interactions and collisionless inertial effects. Here, one example of each will be suggested as plausible mechanisms for explaining the observed non-classical ion heating in the null-helicity reconnection environment of MRX.

The lower-hybrid drift instability [DAvidson and GLADD, 1975] is a high frequency $\left(\Omega_{\mathrm{i}} \ll \omega \ll \Omega_{\mathrm{e}}\right)$ mode driven unstable by cross-field current and associated pressure gradients, and it persists in the regime $T_{\mathrm{e}} \lesssim T_{\mathrm{i}}$ as required in MRX. This

\footnotetext{
${ }^{24}$ This is Troy Carter's dissertation research.

${ }^{25}$ See e.g. [Coroniti, 1985] for a good discussion.
} 
mode has been proposed to explain satellite measurements of electrostatic and magnetic fluctuations in the magnetotail [HuBA et al., 1978]. Recently, this mode has been analyzed in the context of MRX [CARTER et al., 1999], and a linear analysis shows marginal stability at $V_{\mathrm{dr}} \equiv j_{\theta} / n e$ equal to $v_{\mathrm{th}, \mathrm{i}}$ and a very fast growth rate equal to the lower hybrid frequency $(\sim 30 \mathrm{MHz})$. The observation of a constant $V_{\mathrm{dr}} / v_{\mathrm{th}, \mathrm{i}}$ over a wide range of densities in MRX [JI et al., 1999] is suggestive of the current being limited at marginal stability by a current-driven instability. The proposed mode, which propagates at an angle of $\left(m_{\mathrm{e}} / m_{\mathrm{i}}\right)^{1 / 2}$ with respect to the current, is driven by electron Landau damping because it is a negative energy mode. Since the wave can Landau damp simultaneously on electrons and ions, this could lead to direct ion heating. Preliminary estimates show that it is plausible for this mode to explain the enhanced resistivity and the ion heating observed in MRX [KulsRud and CARTER, 2000]. This is an electrostatic mode which is stabilized at high- $\beta$ and is expected to operate only at the edges of the current sheet (since $\beta$ is large near the field null), seemingly reducing its effectiveness. However, preliminary indications in 3-D simulations are that the mode can propagate into the center of the reconnection layer nonlinearly [RoGERs et al., 1999].

Another possible ion heating mechanism is suggested in [SHAY et al., 1998]. This work uses a 2.5-D hybrid code (kinetic ions, fluid electrons) to study collisionless reconnection including Hall dynamics and electron inertia. In this simulation, a twoscale structure develops in the reconnection layer owing to the Hall effect, which allows ions and electrons to decouple on scale lengths shorter than $c / \omega_{\mathrm{pi}}$, the scale at which ions become unmagnetized. (MRX data also shows a current sheet thickness of $\left.c / \omega_{\mathrm{pi}}.\right)$ The electrons continue inward into the layer until the $c / \omega_{\mathrm{pe}}$ scale at which they finally become unmagnetized and the flux-freezing constraint is finally broken. A self-consistent electric field arises between the $c / \omega_{\mathrm{pi}}$ and $c / \omega_{\mathrm{pe}}$ scales due to the charge separation and can accelerate inflowing ions up to the Alfvén speed. The counter-streaming ions which come in from both sides of the layer mix and appear to be heated instantaneously. This would seem to be consistent with the immediate rise in $T_{\mathrm{i}}$ observed in the center of the reconnection layer in MRX. However, when 3-D effects are included in the simulations, the thin electron layer breaks up turbulently [DRAKE et al., 1997], and it is not clear if this ion heating mechanism would survive in a physical reconnection layer. 
Two examples have been mentioned as candidates for the observed ion heating in MRX. Clearly, this area is forefront research, and answers are still forthcoming.

\subsection{Summary}

In this chapter, local ion temperature and flow measurements from a well characterized reconnection layer were presented. First, a rise in $T_{\mathrm{i}}$ during reconnection was identified, for both null-helicity $(6 \rightarrow 17 \mathrm{eV})$ and co-helicity $(3 \rightarrow 7 \mathrm{eV})$ reconnection. The $T_{\mathrm{i}}$ rise was then causally linked to the existence of reconnection, i.e. the $T_{\mathrm{i}}$ rise occurred only when reconnection was driven, and furthermore the magnitude of the rise was in direct proportion to the amount of magnetic field energy dissipated. Additionally, the rise in $T_{\mathrm{i}}$ was also shown to be localized spatially in the region of the reconnection current sheet and magnetic field reversal. These results collectively could be interpreted as the first clear demonstration of ion heating due to magnetic reconnection.

Local downstream ion flow measurements showed the flows to be small, at most $25 \%$ of $V_{\mathrm{A}}\left(0.25 V_{\mathrm{A}} \approx 8 \mathrm{~km} / \mathrm{s}\right)$. This observation is consistent with the high downstream pressure observed in MRX, postulated to arise due to fast pressure equalization in the finite downstream volume. The small flow speeds of MRX are in direct contrast to the results of TS-3 [ONO et al., 1996; ONO et al., 1997], as well as to classical MHD reconnection theories which did not account for the effects of a non-negligible downstream pressure. Toroidal flows were also measured and shown to be small (a few $\mathrm{km} / \mathrm{s}$ at most) and not a possible energy source for the observed ion heating. The toroidal flow measurements are not consistent with diamagnetic ion drift, which is an order of magnitude larger than the measured value, but the discrepancy may be related to the existence of a radial electric field which causes an $E \times B$ drift modifying the diamagnetic drift. This needs further investigation.

An energy budget for the reconnected field and ion energy was considered. The analysis showed that substantial ion heating occurred and that approximately $65 \%$ of the reconnected field energy was converted to ion thermal energy. Without the action of non-classical heating mechanisms, at most only about $17 \%$ of the dissipated field energy would have been converted to ion energy via classical viscosity, meaning that $48 \%$ of the energy was converted non-classically. Finally, there is a 
hint of stronger ion heating with increased resistivity enhancement, suggesting a relationship between the non-classical mechanisms responsible for enhanced resistivity and for ion heating.

The exact mechanism for the heating is still an open question and is the subject of ongoing research, both experimentally and theoretically. Conversely, the identification of non-classical ion heating on MRX should impact current thinking on the possible non-classical micro-physics in the reconnection layer. 


\section{Chapter 5}

\section{Conclusions and Future Work}

$\mathrm{I}$

ON HEATING AND ACCELERATION during magnetic reconnection have been investigated experimentally in a controlled laboratory experiment. The netic field topology during reconnection to the measurements of local $T_{\mathrm{i}}$ in the reconnection layer, have led to a more comprehensive understanding of the reconnection process, which is often postulated but had never been shown in detail to heat and accelerate ions. The experimental identification of non-classical ion heating during magnetic reconnection is a significant result which can potentially impact many research disciplines.

\subsection{Conclusions}

The main result of this research is the local measurement of non-classical ion heating during fully diagnosed magnetic reconnection events. One of the guiding philosophies in this work was to listen to the plasma, and to consider and learn from the implications of theory but not to rely on theory. This is because a comprehensive experimental picture of reconnection in high Lundquist number $(S \gg 1)$ plasmas (relevant to reconnection processes of interest in nature and in laboratory plasmas) had not been produced prior to this work. ${ }^{1}$ Relevant quantities were measured as much as possible in developing a physical understanding of the reconnection and ion heating process.

\footnotetext{
${ }^{1}$ University of Tokyo experiments [ONO et al., 1997] are producing results in parallel with MRX.
} 
A 2-D and quasi steady-state reconnection layer was formed and studied. The layer is toroidal so there are no end effects. The 2-D and quasi steady state properties of the layer allowed clear interpretation of the data and easy comparison with established theories. Detailed magnetic field measurements showed the time evolution and detailed spatial characteristics of the reconnection layer. Highlights of MRX reconnection physics results, discussed in Sec. 3.7, include the following. It was found that the inclusion of an out-of-plane magnetic field significantly altered the structure of the layer (forming O-points) as well as the reconnection speed (decreasing it by a factor of three) [YAmADA et al., 1997b]. The Sweet-Parker model was tested experimentally for the first time. Consideration of the measured enhanced plasma resistivity, plasma compressibility, and pressure profile along the layer led to a modified Sweet-Parker picture which correctly predicted the observed reconnection rate [JI et al., 1998; Ji et al., 1999]. Finally, the equilibrium profile of the reconnecting current sheet was seen to agree with the oft-quoted Harris solution for a collisionless current sheet [HARRIS, 1962], and the thickness of the current sheet was seen to scale with ion skin depth $c / \omega_{\mathrm{pi}}$ [YAMADA et al., 2000].

For the ion heating study, the goal at the outset of this work was to determine conclusively if ions are heated directly due to the reconnection process. Many previous experiments obtained ion heating results with varying degrees of success and relevance to reconnection in plasmas with $S \gg 1$. The most comprehensive picture was obtained on TS-3 at the University of Tokyo [ONo et al., 1996; Ono et al., 1997], which reported acceleration of ions to super-Alfvénic speeds via a "slingshot" effect (tension force) of reconnected field lines, and then subsequent viscous thermalization of this flow to explain the measured substantial global ion heating. As discussed in Sec. 1.3.3, reconnection was likely not the only energy conversion process present in TS-3. In the present experiments, the reconnection process is better isolated from other effects and characterized in greater detail so that clear cause and effect between reconnection and ion heating can be established. The other important new contribution is the direct, local measurement of the majority plasma ion temperature and flow velocity. This was made possible by a novel spectroscopy probe $^{2}$ which could be placed directly in the current sheet and scanned

\footnotetext{
${ }^{2}$ The IDSP (ion dynamics spectroscopy probe) [FIKSEL et al., 1998] was generously loaned to MRX by Dr. Gennady Fiksel of the University of Wisconsin-Madison.
} 
radially. Helium discharges were used so that spectroscopy of the He II $4686 \AA$ line gave direct information on the majority plasma ions.

Based on these new measurements, local ion heating during magnetic reconnection was identified for the first time [Hsu et al., 2000]. The ion temperature rose by approximately a factor of three during null-helicity reconnection and approximately a factor of two in co-helicity reconnection, consistent with the observation that null-helicity reconnection is faster and thus dissipates more magnetic energy. Null-helicity reconnection appears to heat ions more efficiently than co-helicity reconnection since only a factor of two increase in dissipated magnetic energy (compared to co-helicity) resulted in a factor of four larger increase in $T_{\mathrm{i}}$. The resistivity enhancement in null-helicity is observed to be a factor of two larger compared to the co-helicity case, indicating the possible role played by non-classical dissipation mechanisms in heating ions. The ion heating could not be explained by energy exchange with electrons $\left(T_{\mathrm{e}} \approx 10-15 \mathrm{eV}\right)$ since the characteristic ion-electron energy relaxation time is approximately $400 \mu \mathrm{s}$ in helium discharges. The ion heating also could not be explained by viscous heating of flows, which are accelerated to only $0.2 V_{\mathrm{A}}$. An ion energy balance for null-helicity reconnection shows that approximately $48 \%$ of the reconnected field energy is converted to ion thermal energy non-classically, with an additional $17 \%$ converted via classical viscosity. This is supported by observations of resistivity enhancement over the Spitzer value by an order of magnitude.

An experiment was performed to investigate the scaling of ion heating with resistivity enhancement. The results hint at a marginal scaling (error bars are large) between the rise in ion thermal energy normalized by the total reconnected field energy and the resistivity enhancement. If true, this indicates that as collisionless effects become more important, direct heating of ions goes up correspondingly. This suggests a close relationship between the physics of the plasma resistivity (which limits the current and dissipates magnetic energy) and the physics of ion heating (which is due to acceleration and subsequent scattering of ions possibly by wave fields).

The existence of "enhanced plasma resistivity" has been questioned. Contributions of $Z_{\text {eff }}>1$ and electron-neutral collisions to the resistivity have not been 
determined experimentally, although the effects are expected to be small. Furthermore, there is always the question on what "Spitzer resistivity" ought to be in MRX. ${ }^{3}$ However, the observed ion heating can be taken as proof that non-classical mechanisms are important in MRX, for none of the above effects can produce the observed ion heating. The exact mechanism in the reconnection layer responsible for the heating is an open question. The possibilities include wave-particle interactions and inertial effects, as discussed in Secs. 4.3.6 and 4.5. It is possible that current-driven instabilities may produce the observed heating; this mechanism has been investigated theoretically [KULSRUD and CARTER, 2000], and efforts to identify fluctuations in the relevant range of frequencies is underway experimentally [CARTER et al., 1999]. Straightforward inertial effects, e.g. due to Speiser orbits [SPEISER, 1965], are unlikely to be important in MRX because even Coulomb collisions occur more frequently. However, a self-consistent inertial model and simulations based on "whistler mediated" reconnection [MANDT et al., 1994; SHAY et al., 1999] are not inconsistent with ion heating observations in MRX. In this model, separation of charge on scales smaller than $c / \omega_{\text {pi }}$ leads to ambipolar fields which can accelerate ions effectively. Scales smaller than $c / \omega_{\text {pi }}$ are difficult to diagnose in MRX, but an electrostatic probe array with better than $1 \mathrm{~cm}$ spatial resolution may be able to distinguish such a potential well. However, in $3-\mathrm{D}$, the simulation results show turbulent breakup of the smaller scale, and it is unclear whether the ambipolar fields survive.

Finally, an example of how the findings of this dissertation might impact another research discipline is given. Typically, when a model of coronal activity invokes reconnection, it almost always assumes acceleration of particles to very high energies. This prompts researchers to look for tell-tale signs of high energy particles colliding with other material, which would give off hard X-rays and other energetic radiation, as a signature of reconnection. Thus, if hard X-rays or other energetic radiation are not seen, it might be assumed that there was no reconnection. The MRX results suggest the possibility of "quiet" reconnection which would be very difficult to detect via observations. Yet ions could be continuously heated, and the source of the heating would be virtually undetectable (since detailed measurements of magnetic field in the corona are unavailable). This scenario could be relevant to the problem

\footnotetext{
${ }^{3}$ This question has been addressed in part in [KULSRUd, 1997].
} 
of coronal heating, see e.g. [Golub and PAsACHOFF, 1997].

\section{$5.2 \quad$ Future work}

Much work remains to be done to fully understand the mechanisms of the nonclassical ion heating process in MRX. It is expected that these mechanisms are very closely related to (if not the same as) the dissipation mechanisms giving rise to enhanced values of plasma resistivity. Other than the obvious next step of examining the frequency spectrum of turbulent fluctuations in hopes of identifying relevant modes (this is already underway), ${ }^{4}$ the most effective ways of continuing this study would be to improve the spatial and temporal resolution of the $T_{\mathrm{i}}$ measurement and to expand the possible operational regime by improving the vacuum quality of MRX and to alter boundary condition effects. These capabilities will all be available in the near future.

Phenomena on scales smaller than $5 \mathrm{~cm}$ and shorter than $10 \mu$ s could not be addressed due to physical limitations of the IDSP and available plasma light. Small spatial-scale and fast time-scale information could hold very important clues regarding the non-classical dissipation mechanisms. For example, $T_{\mathrm{i}}$ anisotropy would focus attention on either parallel or perpendicular heating mechanisms. IDSP measurements suggest that $T_{\mathrm{i} \perp} \approx T_{\mathrm{i} \|}$, but this information is limited because $10 \mu \mathrm{s}$ is enough time for $T_{\mathrm{i}}$ to isotropize if it started out anisotropic. Also, various proposed non-classical mechanisms are sensitive to $\beta$, which for null-helicity reconnection might lead to local heating at the edge of the reconnection layer where $\beta$ is small. This requires spatial resolution of better than $1 \mathrm{~cm}$, which is impossible for the IDSP. A laser-induced fluorescence system is scheduled to be installed in the near future onto MRX [TRINTCHOUK et al., 1999], which could potentially yield full ion distribution functions with excellent spatial $(<1 \mathrm{~cm})$ and temporal $(<100 \mathrm{~ns})$ resolution. A different working gas (Ar) or impurities may need to be used for the LIF measurements, but this could also give new insight into the physics.

Improved vacuum quality could result in a factor of four increase in $T_{\mathrm{e}}$. The plasma would become truly collisionless with $\lambda_{\text {mfp }}$ equal to or larger than the machine size. Radiative losses would be lower. It would be highly desirable to study

\footnotetext{
${ }^{4}$ The subject of T. A. Carter's dissertation research.
} 
the reconnection and heating physics in these regimes and be able to obtain improved scaling relations between, for example, heating and reconnection rate versus resistivity enhancement and Lundquist number.

The development of a third capacitor bank will allow studies of "push" reconnection, as described in Sec. 2.2, by separating the plasma formation phase from the push reconnection phase. Push reconnection would allow studies of counter-helicity reconnection, also described in Sec. 2.2, in which a sheared out-of-plane field exists in the layer. In this case, toroidal field is reconnected, as in TS-3, and larger ion heating may be observed. Furthermore, the buildup of downstream pressure may be modified in push reconnection because the global geometry would be changed, with the reconnection layer oriented along $R$ rather than $Z$. Since the downstream region would have a larger volume in push reconnection, the downstream pressure may not be significant during the reconnection phase, and it may be possible for Alfvénic flows to develop, which could lead to significantly different reconnection and heating dynamics.

Ultimately, the correlation of ion heating with fluctuations and, hopefully, the identification of a wave dispersion relation is the goal for achieving a more complete understanding of the reconnection (dissipation of magnetic energy) and ion heating processes in MRX. 


\section{Appendix A}

\section{Independent Check of Mach Probe Measurements}

$\mathrm{I}$ ON FLOW SPEEDS reported in this dissertation were obtained mainly using Mach probes. An unmagnetized fluid sheath model [HUDIs and LIDSKY, interpret the Mach probe data. Due to the complexity of plasma sheath physics, Mach probe theories are commonly believed to be correct to within only a factor of two. However, the availability of IDSP (see Sec. 2.3.3) Doppler shift data afforded a unique opportunity to "calibrate" the Mach probe. This appendix chapter describes an independent check of the Mach probe measurements using the IDSP. It is shown that the accuracy of the generalized Hudis and Lidsky model is sufficient to support the claim that downstream ion flow speeds are only $25 \%$ of the Alfvén speed.

In performing the independent check, toroidal ion flow speed $V_{\theta}$ in the reconnection region is measured using both the IDSP and the Mach probe, and the results are compared to each other. Data are taken at two spatial positions, $R=37.5$ and $42.5 \mathrm{~cm}$. Multiple shots are required to obtain a $V_{\theta}$ value using the IDSP since only one sightline is used. Approximately ten shots are fired with the IDSP sightline facing the $-\theta$ direction, and an average and standard deviation for the location (in CCD pixels) of the peak of the spectrum are obtained. This is repeated for the sightline facing the $+\theta$ direction. The difference between the two central pixel numbers translates into a net relative Doppler shift $\Delta \lambda$ between the emission lines collected by the two opposing sightlines. It is straightforward to show (referring to 
Fig. 2.15) that

$$
V_{\theta}=c \frac{\Delta \lambda}{\lambda_{0}} \frac{1}{2 \cos (\phi)}
$$

where $c$ is the speed of light, $\lambda_{0}=4686 \AA$ is the unshifted wavelength of the spectral line, and $\phi=45^{\circ}$ is the angle between the sightline and the toroidal flow. Thus, all twenty shots are used to obtain an averaged $V_{\theta} \pm \delta V_{\theta}$ at each radial location using the IDSP. Standard error propagation using the standard deviations of the peak locations for each group of ten shots gives the representative shot-to-shot error $\delta V_{\theta}$. A value for $V_{\theta}$ is also obtained for each plasma shot using the Mach probe according to

$$
V_{\theta}=\sqrt{\frac{T_{\mathrm{e}}}{T_{\mathrm{i}}}} \sqrt{\frac{T_{\mathrm{e}}}{m_{\mathrm{i}}}} \tanh ^{-1} K,
$$

as described in Ch. 2, where $K$ is the difference in ion saturation currents divided by the sum. The corresponding values for $T_{\mathrm{e}}$ and $T_{\mathrm{i}}$ for each shot are taken from triple Langmuir probe and IDSP measurements, respectively.

Results from both the IDSP and Mach probe are shown in Fig. A.1. Error bars in the ordinate represent \pm one standard deviation in the shot-to-shot variation of a group of twenty shots. Error bars in the abscissa represent the spatial extent for the IDSP and the uncertainty in measuring the location of the Mach probe. From the figure, it is seen that the Mach probe measurement is about twice the IDSP value at $R=37.5 \mathrm{~cm}$ and only about 1.3 times the IDSP value at $R=42.5 \mathrm{~cm}$. In both cases, the error bars overlap significantly, and the Mach probe value overestimates the IDSP value. Given the general accuracy of Mach probe theories, this agreement can be considered to be better than average, and it certainly validates the values of ion flow reported in Ch. 4, which include an approximate calibration factor of 0.7 .

The fact that IDSP values are consistently smaller than Mach probe values might be explained by an averaging effect of the IDSP measurement, which may underestimate the true flow speed when there is a local gradient in the flow. 


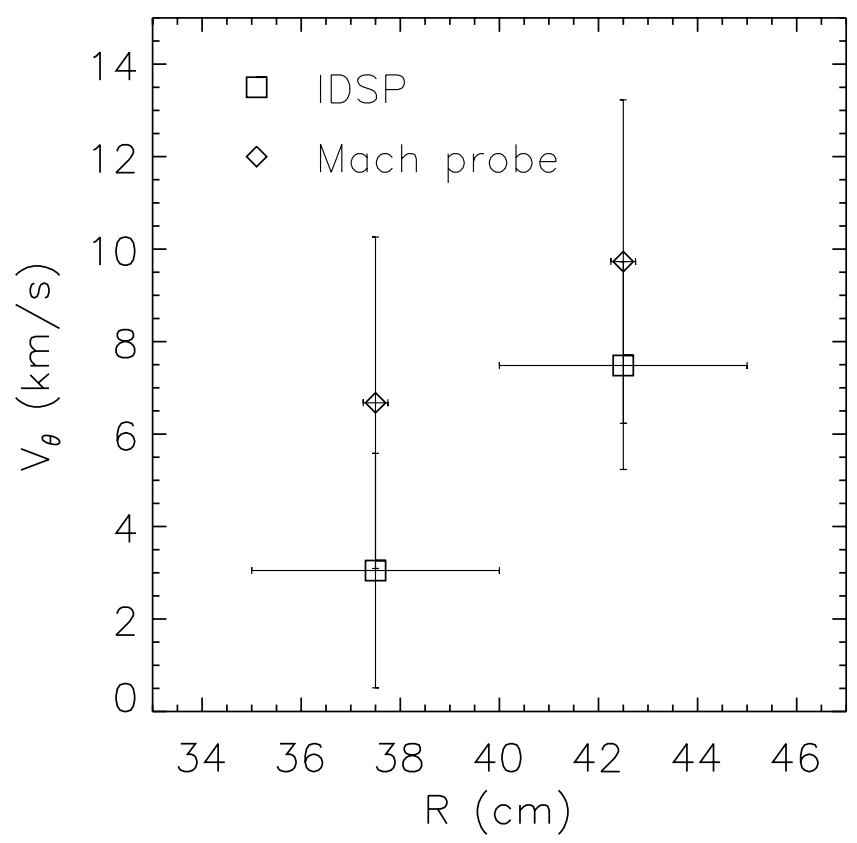

Figure A.1: Comparison of $V_{\theta}$ measurements using IDSP Doppler shift and Mach probe based on an unmagnetized fluid sheath model (shots 12187-12232). Data are for $Z=0 \mathrm{~cm}$ and $t=260 \rightarrow 270 \mu \mathrm{s}$. 


\section{Appendix B}

\section{Plasma Perturbation due to the IDSP}

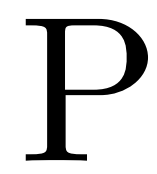

ERTURBATION of the plasma by probes must be considered in interpreting the experimental data. The IDSP is by far the most bulky and likely the "dirtiest" probe inserted into MRX. This appendix chapter focuses on the effects of the IDSP on magnetic field topology, density and electron temperature, and global ion emission. ${ }^{1}$

The basic procedure is to scan the IDSP radially and simultaneously monitor the magnetic probe arrays, triple Langmuir probe, and global ion line emission. It is found that the IDSP does not affect these measurements in any significant way, as long as approximately fifteen conditioning discharges are fired first. Before the discharge cleaning, the electron temperature is reduced from normal levels by a factor of two, and He II $4686 \AA$ emission is virtually undetectable. It is believed that the conditioning discharges rid the IDSP boron nitride surfaces of adsorbed impurities.

Figure B.1 shows the poloidal magnetic field during pull reconnection with the IDSP far outside the reconnection region $(R=52.5 \mathrm{~cm})$ and inside the reconnection region $(R=37.5 \mathrm{~cm})$. The vector plots are averaged over five shots for each case. As seen in the figure, the magnetic topology is not significantly different

\footnotetext{
${ }^{1}$ The effects of magnetic and electrostatic probes have also been studied in the past. The presence and positions of these probes generally have no detectable effects on other measurable quantities beyond the standard shot-to-shot statistical variation. On occasion, significant deviations in a large number of probe signals may occur simultaneously due to ground loop noise pickup or an arc inside the vacuum chamber. These discharges are rejected.
} 


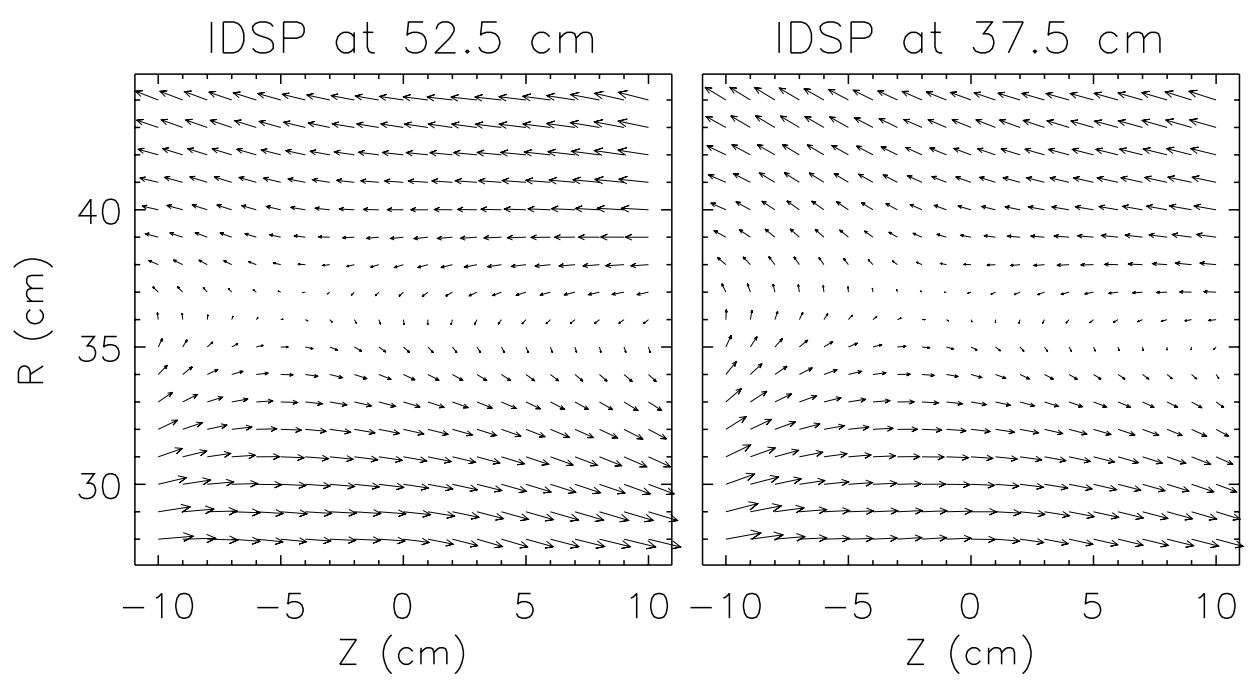

Figure B.1: Poloidal magnetic field measurements (for $t=265 \rightarrow 275 \mu \mathrm{s}$ ) for two IDSP positions (shots 11479-83 and 11494-98).

between the two cases; in fact, observable differences are not unlike typical shotto-shot variations. However, the total toroidal current calculated from the field measurements is about $20 \%$ smaller when the IDSP is at $R=37.5 \mathrm{~cm}$. This is still within typical shot-to-shot variation, but it is an indication that the IDSP may affect current sheet formation. Placement of the IDSP in the reconnection layer does not alter global reconnection dynamics. However, in comparing shots with and without the IDSP in the current sheet, the slight difference in toroidal current should be taken into account.

Figure B.2 shows triple Langmuir probe data at $R=37.5 \mathrm{~cm}$ and $Z=0 \mathrm{~cm}$ for different IDSP radial positions. Discharge parameters are held constant (TF/PF $=13 / 11 \mathrm{kV}$, initial gas pressure $=6 \mathrm{mT}$, null-helicity). It can be seen that $T_{\mathrm{e}}, n_{\mathrm{e}}$, and the probe potential $V_{\mathrm{f}}$ remain constant (within error bars) as the central IDSP position is varied from $R=32.5 \rightarrow 52.5 \mathrm{~cm}$. This data shows that basic plasma parameters inside the reconnection region are unchanged due to the presence and position of the IDSP.

Figure B.3 shows the brightness and broadening of chord-averaged He II $4686 \AA$ line emission as a function of IDSP radial position. The viewing chord is approximately $2.5 \mathrm{~cm}$ in diameter and is tangent to $R \approx 40 \mathrm{~cm}$. The chord spans the entire vacuum chamber at $Z=0 \mathrm{~cm}$. As seen in the figure, both the brightness 


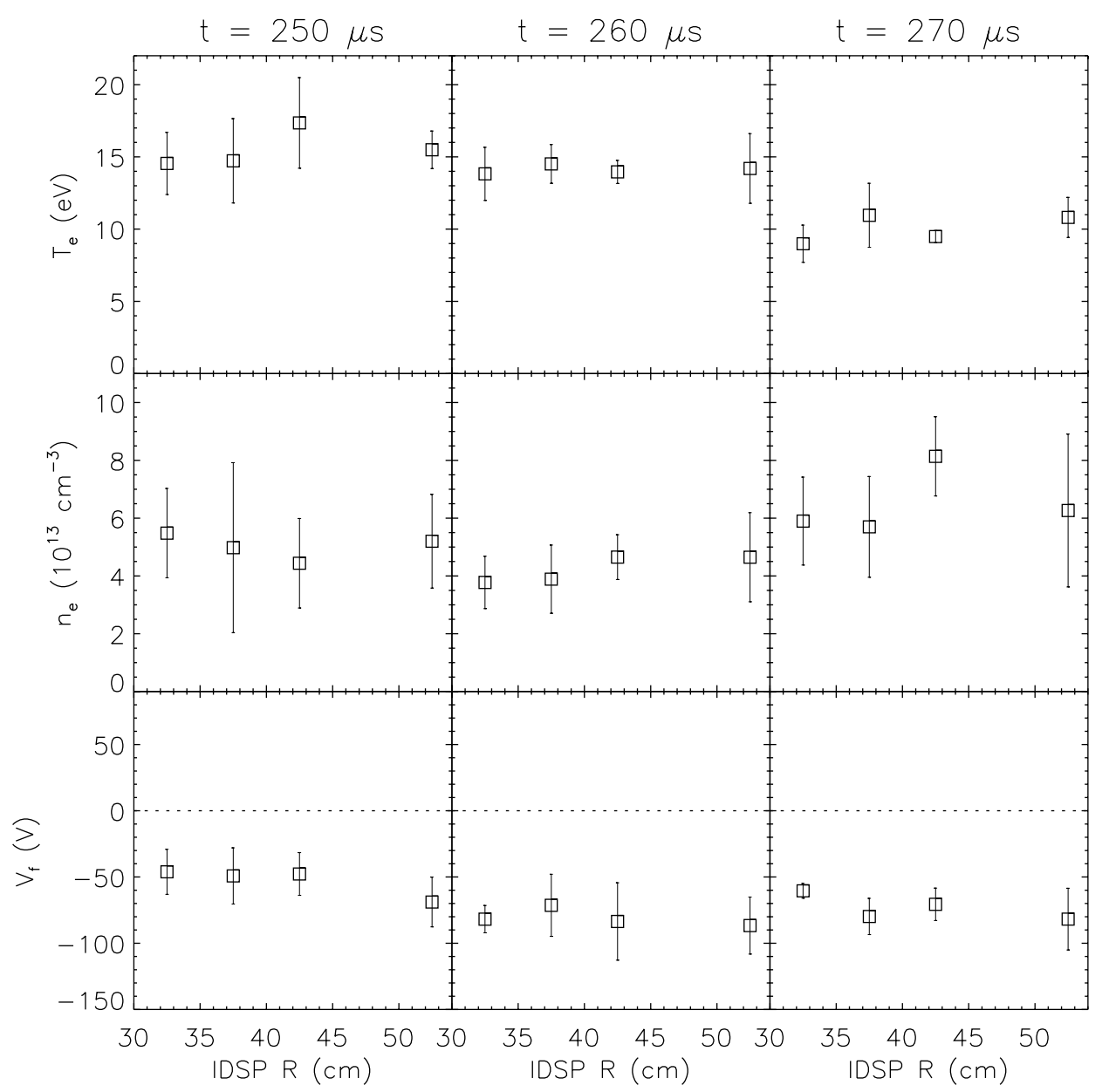

Figure B.2: Triple Langmuir probe measurements (at $R=37.5 \mathrm{~cm}$ and $Z=$ $0 \mathrm{~cm}$ ) as a function of IDSP radial position (shots 11479-11503). For reference, the reconnection layer typically is centered at $R=37.5 \mathrm{~cm}$.

and the broadening of the emission are approximately constant within error bars as the IDSP is scanned radially, meaning that global ion properties are essentially unaffected due to the presence and position of the IDSP. However, it is interesting to note that both the intensity and line broadening suffer a slight dip when the IDSP is located at $R=32.5 \mathrm{~cm}$. This is likely due to the fact that the main bulk of the IDSP housing sits in the current sheet when the viewing position is $R=32.5 \mathrm{~cm}$ (see Figs. 2.15 and 2.16 for reference). Therefore, the overall density and ion temperature may be reduced when the probe housing sits squarely in the path of the current sheet. This result should be considered in interpreting IDSP 


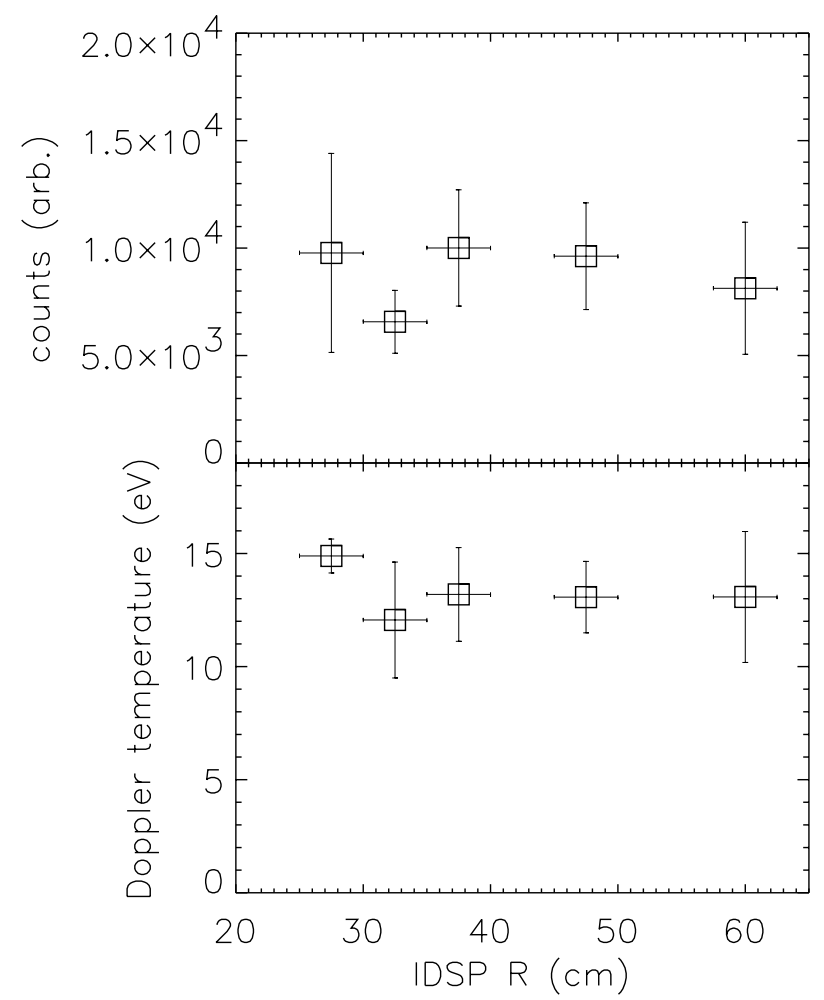

Figure B.3: (top) Brightness and (bottom) Doppler temperature of chord-averaged He II $4686 \AA$ emission. Viewing chord is tangent to $R \approx 40 \mathrm{~cm}$, and CCD gate opening is $t=260-270 \mu$ s (shots 12127-12154).

data.

Overall, it is concluded that systematic studies of reconnection in MRX are still meaningful with the IDSP placed in the current sheet. The small effects on total toroidal current and the reduction in ion light emission when the IDSP is at $R=32.5 \mathrm{~cm}$ must be taken into account properly when data interpretation calls for it. 


\section{Appendix C}

\section{Doppler spectroscopy of helium ion spectral lines}

I ON TEMPERATURE measurements are based on Doppler spectroscopy of the He II $4686 \AA$ ion spectral line. Two key factors contributed to very clean data interpretation and hence robust physics conclusions. First, plasma light was collected from a localized region using the novel IDSP diagnostic [FIKSEL et al., 1998], which is described in Sec. 2.3.3. This contrasts with the usual method of collecting plasma light along a chord spanning most of the plasma. The only way to deduce localized $T_{\mathrm{i}}$ using the latter method would be to utilize many such chords and apply mathematical inversion techniques requiring varying levels of assumptions such as axisymmetry, closed flux surfaces, etc. While this method is certainly possible and has yielded many successful measurements of density and poloidal flow (in tokamaks for example), it is extremely difficult. Some of the necessary hardware for this multi-chord scheme was developed as backup for this work, and hopefully it will be utilized in the future. The second factor is the use of pure helium discharges for this set of experiments. Use of pure helium means that the Doppler temperature deduced from He II 4686 A spectra is the majority ion temperature and that it is not necessary to rely upon details about charge exchange (necessary for neutral spectroscopy) nor energy transfer between impurity and majority ions (necessary for impurity ion spectroscopy). Details and limitations of the diagnostic hardware, including the IDSP, the fiber optics, the spectrometer, and the CCD camera are described in Sec. 2.3.3. Here, the emphasis is on deducing $T_{\mathrm{i}}$ from raw spectra. 


\section{C.1 Principle of Doppler spectroscopy}

Via the Doppler effect, the width of a spectral line will in general be broadened by the motion of emitters along the measurement line-of-sight; this is known as Doppler broadening. If Doppler broadening is the dominant effect on the spectral line shape and if the emitter line-of-sight velocity distribution is thermal with temperature $T$, then the resulting wavelength spectrum will be a Gaussian centered at the unshifted line wavelength $\lambda_{0}$. In this case, it is straightforward to deduce $T$ from the full width half maximum (FWHM) of the measured spectrum $\Delta \lambda_{\mathrm{FWHM}} \cdot{ }^{1} \mathrm{~A}$ convenient expression is

$$
T=1.69 \times 10^{8} \mu\left(\frac{\Delta \lambda_{\mathrm{FWHM}}}{\lambda_{0}}\right)^{2} \mathrm{eV},
$$

where $\mu$ is the mass of the emitter divided by the proton mass.

If the thermal emitters have a uniform bulk flow velocity $V$ along the line-ofsight, then the measured wavelength spectrum will still be Gaussian but Doppler shifted to a central wavelength $\lambda_{0}^{\prime}=\lambda_{0}(1+V / c)$, where $c$ is the speed of light and $V>0$ refers to flow away from the viewing point. Thus, $V$ can be determined,

$$
V=c\left(\frac{\lambda_{0}^{\prime}-\lambda_{0}}{\lambda_{0}}\right)
$$

if $\lambda_{0}^{\prime}-\lambda_{0}$ is known absolutely. In this case, $\lambda_{0}$ in Eq. (C.1) must be replaced by $\lambda_{0}^{\prime}$, but this an imperceptible difference for $|V| \ll c$.

Note the two critical assumptions implicit in Eq. C.1: (1) Doppler broadening is the dominant effect on the line shape and (2) the emitter line-of-sight velocity distribution is thermal (with or without $V$ ). These points will be addressed in the next section.

\footnotetext{
${ }^{1}$ These elementary points are discussed in detail in plasma spectroscopy and diagnostic textbooks such as [GRIEM, 1964; WIESE, 1965].
} 


\section{C.2 Other line broadening mechanisms}

Typically, measured spectra are not perfect Gaussians because Doppler broadening is not the only physical effect which determines the spectral line shape. This section discusses other effects and methods to overcome them if they are not negligible. The discussion will focus solely on the He II $4686 \AA$ line since that is the line on which all the $T_{\mathrm{i}}$ data in this chapter are based.

\section{C.2.1 Instrumental broadening}

The instruments used for spectral measurements introduce unavoidable wavelength dispersion in the collected light. This is known as instrumental broadening. Because the instrumental broadening and Doppler broadening are independent processes, the resulting spectrum which is ultimately recorded is a convolution of the two independent spectra. Therefore, if the instrumental profile is known accurately, the Doppler profile can be deduced using numerical de-convolution techniques. The instrumental broadening needs to be small compared to the Doppler broadening to yield very accurate values of $T_{\mathrm{i}}$.

The instrumental profile can be determined by coupling light from a helium lamp into the spectrometer. Because the lamp is cold, only neutral helium lines are available. In general, the wavelength dispersion of the spectrometer is a function of wavelength. However, there is a helium neutral line at $4713 \AA$, very close to the ion line of interest at $4686 \AA$, and thus it is reasonable to use the instrumental profile at $4713 \AA$ for de-convolution.

An example of the instrumental profile is shown in Fig. C.1. This profile was obtained using a spectrometer slit width of $100 \mu \mathrm{m}$, the same setting used throughout these experiments. The profile has a FWHM of approximately 5 CCD pixels, which corresponds to $0.37 \AA$. Using Eq. (C.1), it can be seen that this is equivalent to a temperature of $5 \mathrm{eV}$, meaning that de-convolved $T_{\mathrm{i}}$ values below approximately $5 \mathrm{eV}$ will have large relative errors. The measured instrumental profile is used in the curve-fitting and de-convolution procedures to be discussed in Sec. C.3. 


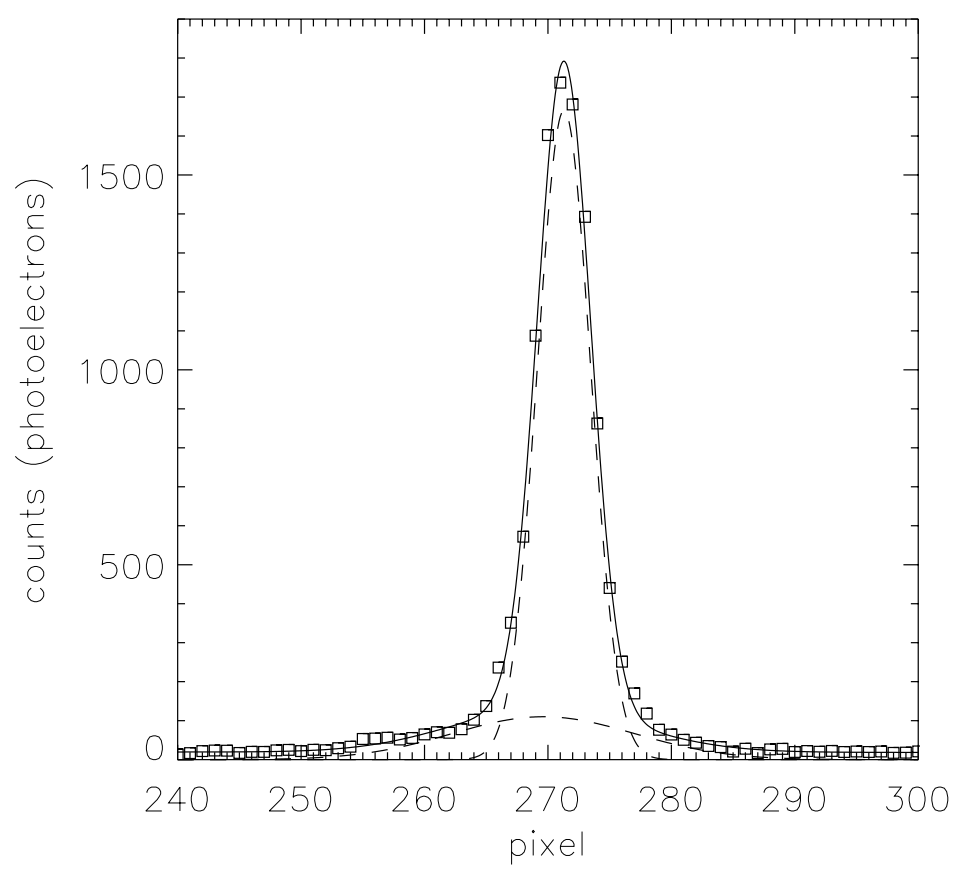

Figure C.1: Instrumental broadening profile of the HeI $4713 \AA$ spectral line from a helium lamp. Data points (squares) are fit to a sum (solid line) of two Gaussians (dashed lines).

\section{C.2.2 Fine structure}

The He II $4686 \AA$ line consists of thirteen fine structure components of varying intensities and spanning $0.5 \AA$. These have been characterized using a helium lamp [CHIU, 1995], albeit at different pressures and temperatures from MRX. Two components, which are separated by only $0.1 \AA$, are approximately an order of magnitude stronger than all the others and thus dominate the total spectrum. These two components should undergo the same Doppler broadening, and thus the fine structure effects can be modeled to lowest order using two equal-temperature Gaussians separated by $0.1 \AA$. Since the expected Doppler broadening is up to approximately $1 \AA$, the difference in temperature obtained using one versus two Gaussians is small $(\approx 10 \%)$, certainly much smaller than the typical shot-to-shot variation $(\approx 30 \%)$. Therefore, all reported values of $T_{\mathrm{i}}$ are based on single Gaussian fits. 


\section{C.2.3 Pressure broadening}

The perturbation of emitter energy levels due to emitter interactions with surrounding particles is known as pressure broadening, which in general includes resonance (interaction with atoms of the same kind), Van der Waals (interaction with atoms and molecules of different kinds), and Stark (interaction with charged particles) broadening. In a plasma where long-range Coulomb forces dominate, Stark broadening is usually the strongest pressure broadening mechanism. ${ }^{2}$ Thus, the goal here is to compare the magnitude of Stark broadening with the overall broadening of the observed He II $4686 \AA$ spectra.

A relationship between the full Stark width $\Delta \lambda_{\mathrm{S}}$ and the electron density $n_{\mathrm{e}}$ for hydrogen and hydrogenic (such as singly ionized helium) lines can be expressed as

$$
n_{\mathrm{e}}=C\left(n_{\mathrm{e}}, T_{\mathrm{i}}\right) \Delta \lambda_{\mathrm{S}}^{3 / 2},
$$

with $n_{\mathrm{e}}$ in $\mathrm{cm}^{-3}$ and $\Delta \lambda_{\mathrm{S}}$ in $\AA .^{3}$ The density and temperature regime of MRX ( $n_{\mathrm{e}} \approx 5 \times 10^{13} \mathrm{~cm}^{-3}$ and $T_{\mathrm{i}} \approx 10-20 \mathrm{eV}$ ) is considered to be beyond the range in which Stark broadening is important for He II $4686 \AA$, and the closest regime for which a numerical value of $C\left(n_{\mathrm{e}}, T_{\mathrm{i}}\right)$ is given in [GRIEM, 1964] is $n_{\mathrm{e}}=10^{15} \mathrm{~cm}^{-3}$ and $T_{\mathrm{i}}=4 \mathrm{eV}$. In this case, $C\left(n_{\mathrm{e}}, T_{\mathrm{i}}\right)=4.34 \times 10^{16} \AA^{-3 / 2} \mathrm{~cm}^{-3}$, corresponding to $\Delta \lambda_{\mathrm{S}}=0.081 \AA$, almost an order of magnitude smaller than the expected Doppler broadening. And in the MRX regime, where $n_{\mathrm{e}}$ is more than an order of magnitude less and $T_{\mathrm{i}}$ a factor of $3-5$ higher, $\Delta \lambda_{\mathrm{S}}$ should be at least another order of magnitude smaller and is therefore completely negligible.

\section{C.2.4 Plasma turbulence}

In certain situations, it is possible for turbulent ion flows to produce a spectrum similar to one produced by random thermal motion. In this case, ion temperature would be overestimated. The most robust method for eliminating this possibility is to obtain the full ion distribution function with sufficient spatial $(<1 \mathrm{~cm})$ and

\footnotetext{
${ }^{2}$ For a detailed account of these matters, the reader is again referred to [GRIEM, 1964; WIESE, 1965].

${ }^{3}$ See Eq. (14-8) and Table 14-1 of [Griem, 1964].
} 
temporal $(<0.1 \mu \mathrm{s})$ resolution, e.g. using laser-induced fluorescence (this measurement is planned in future MRX experiments). The current IDSP measurement has a spatial resolution of approximately $5 \mathrm{~cm}$ and a temporal resolution of $10 \mu \mathrm{s}$, and thus turbulent motion on smaller scales than these can affect the line profile. Some physical arguments will be given on why this is highly unlikely. First, the measured line profiles are extremely close to Maxwellian, as shown in Sec. C.3. It is unlikely that ion flows would conspire to result in a line profile that is so highly Maxwellian. Second, Mach probe measurements of plasma flow, albeit with only incrementally better temporal resolution of $2 \mu \mathrm{s}$, show well-developed and smooth flow patterns that would all but eliminate the necessary gross ion motions needed to produce a false line profile.

\section{C.2.5 Zeeman effect}

Perturbation of emitter energy levels by magnetic fields results in the Zeeman effect. Zeeman shifts are generally on the order of the electron cyclotron frequency. Numerically, the order of magnitude of Zeeman shifts in a magnetized plasma is $\Delta \lambda \approx 10^{-9} \lambda_{0}^{2} B$, where wavelengths are in $\AA$ and $B$ in $\mathrm{kG}$ [GRIEM, 1964]. For $\lambda_{0}=4686 \AA$ and $B \approx 0.25 \mathrm{kG}$, the Zeeman shift is on the order of $5 \times 10^{-3} \AA$, more than two orders of magnitude smaller than the expected Doppler broadening. Thus, the Zeeman effect can be ignored completely in MRX.

\section{C.3 Curve-fitting of measured spectra}

This section discusses in detail the steps taken to derive $T_{\mathrm{i}}$ from a raw He II $4686 \AA$ data set, an example of which is shown in Fig. C.2 (square data points). The abscissa is the pixel number of the CCD camera, and the ordinate is the number of photoelectrons collected by the CCD intensifier. Each pixel number represents $0.0742 \AA$, the calibration of which is described in Sec. 2.3.3. Error bars represent the error in photoelectrons counted according to Poisson statistics (error $=\sqrt{\text { counts }}$ ).

From the preceding section, it was determined that instrumental broadening is the only significant effect other than Doppler broadening in determining the spectral line shape. Therefore, the basic procedure is to fit the data to the convolution of a 


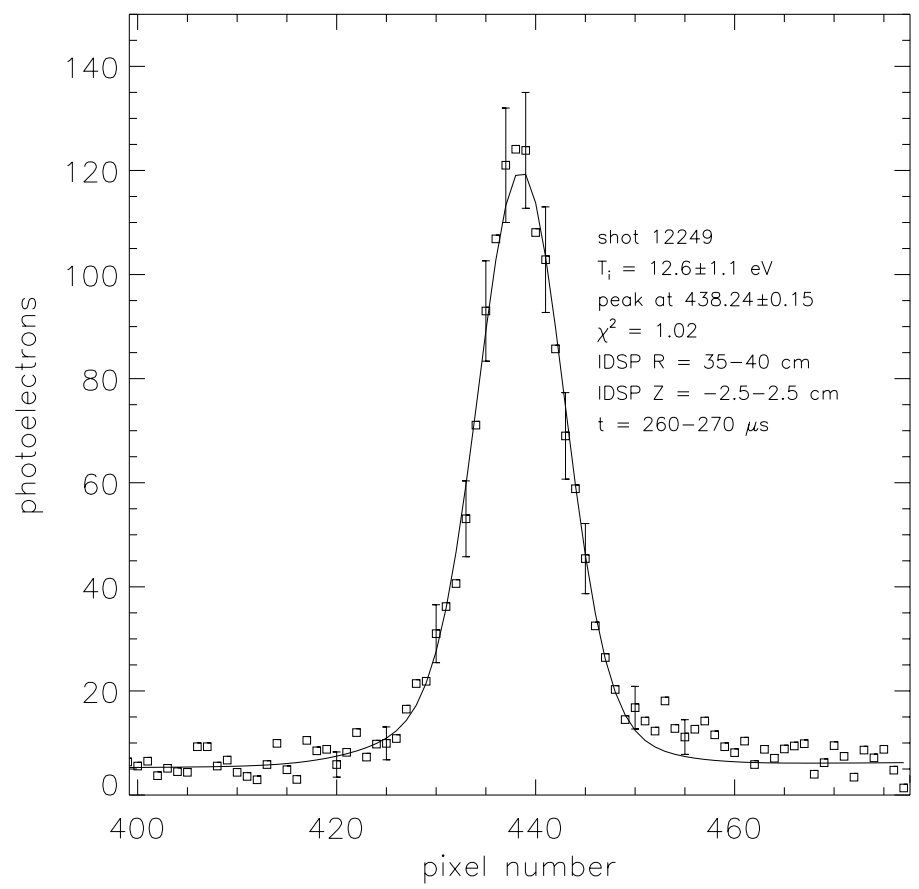

Figure C.2: Doppler broadened He II spectral line, fitted with the convolution of a Gaussian and the instrumental broadening profile.

Gaussian and the instrumental broadening profile iteratively until the reduced- $\chi^{2}$ is minimized.

Curve-fitting is done using two IDL routines. ${ }^{4}$ The first routine (cgauss .pro) generates a single Gaussian or a sum of multiple Gaussians of the form

$$
f(x)=a_{0}+a_{1} x+a_{2} \exp \left[-4 \ln 2\left(\frac{x-a_{3}}{a_{4}}\right)^{2}\right]+a_{5} \exp [\cdots]+\cdots,
$$

where $a_{4}, a_{7}, \ldots$ are the FWHM of each separate Gaussian. The second routine (kurvefit.pro) fits $f(x)$ to the measured spectrum iteratively until the reduced$\chi^{2}$ is minimized and returns the best-fit parameters $\left[a_{0}, \ldots, a_{n}\right]$. The second routine can also fit the data using the convolution of $f(x)$ with any arbitrary function $g(x)$ which can be represented as a sum of Gaussians, provided the fitting parameters

\footnotetext{
${ }^{4}$ The routines cgauss.pro and kurvefit.pro were developed by Dr. R. E. Bell. Both are currently available on the PPPL UNIX cluster in /u/rbell/idllib/.
} 
for $g(x)$ are given.

These two routines are used to determine $T_{\mathrm{i}}$ from a raw spectrum as follows: (1) fit a sum of two Gaussians to the instrumental profile (see Fig. C.1) and retain the best-fit parameters, (2) fit the raw spectrum to the convolution of a single Gaussian $\left[a_{0}, \ldots, a_{4}\right]$ with the instrumental profile (by inputting the previously obtained best-fit parameters), and (3) use Eq. (C.1) to determine $T_{\mathrm{i}}$ from the resultant $a_{4}$, which is the FWHM. The routine kurvefit.pro also returns the fitting error of each parameter $\left[a_{0}, \ldots, a_{4}\right]$ based on the Poisson statistical error of the raw data, indicating a typical error in $T_{\mathrm{i}}$ of approximately $10 \%$. The results of this procedure are shown in Fig. C.2. 


\section{Bibliography}

Anderegg, F., Stern, R. A., Skiff, F., Hammel, B. A., Tran, M. Q., PARIS, P. J., and Kohler, P. (1986). Ion Heating Due to Rotation and Collision in Magnetized Plasma. Physical Review Letters 57, 329.

Baum, P. J. and Bratenahl, A. (1973). Plasma instability at an X-type magnetic neutral point. Physics of Fluids 16, 1501.

Baum, P. J. and Bratenahl, A. (1974). Mass motion and heating in a magnetic neutral point system. Journal of Plasma Physics 11, 93.

Baum, P. J. and Bratenahl, A. (1977). On reconnexion experiments and their interpretation. Journal of Plasma Phys. 18, 257.

Baumjohann, W., Paschmann, G., and Catell, C. A. (1989). Average plasma properties in the central plasma sheet. Journal of Geophysical Research 94, 6597 .

BELL, R. E. (1997). An inversion technique to obtain full poloidal velocity profiles in a tokamak plasma. Review of Scientific Instruments 68, 1273.

Bellan, P. M. (1998). Collisionless reconnection using Alfvén wave radiation resistance. Physics of Plasmas 5, 3081.

Bellan, P. M. (1999). Alfvén -Wave Instability of Current Sheets in Force-Free Collisionless Plasmas. Physical Review Letters 83, 4768.

Bevington, P. R. and Robinson, D. K. (1992). Data Reduction and Error Analysis for the Physical Sciences. WCB/McGraw-Hill, Boston.

Biskamp, D. (1994). Magnetic Reconnection. Physics Reports 237, 179.

Bohm, D., Burshop, E. H. S., and Massey, H. S. W. (1949). The Use of Probes for Plasma Exploration in Strong Magnetic Fields. In The Characteristics of Electrical Discharges in Magnetic Fields, pages 13-76. McGraw-Hill, New York. Edited by A. Guthrie and R. K. Wakerling. 
Braginskit, S. I. (1965). Transport Processes in a Plasma. In Reviews of Plasma Physics Vol. 1, pages 205-311, New York. Consultants Bureau. Edited by M. A. Leontovich.

Bratenahl, A. and Yeates, C. M. (1970). Experimental Study of Magnetic Flux Transfer at the Hyperbolic Neutral Point. Physics of Fluids 13, 2696.

Bretz, N., Jobes, F., and Irby, J. (1997). The design of a second harmonic tangential array interferometer for C-Mod. Review of Scientific Instruments 68, 713.

Burden, R. L. and Faires, J. D. (1993). Numerical Analysis. PWS-Kent Publishing Co., Boston.

Carter, T., Ji, H., Kulsrud, R., Hsu, S., and Yamada, M. (1999). Resistivity and Fluctuations in the Current Sheet of MRX. Bulletin of the American Physical Society 44, 63.

Chen, F. F. (1965). Electric Probes. In Plasma Diagnostic Techniques, pages 113-200. Academic Press, New York. Edited by R. H. Huddlestone and S. L. Leonard.

Chen, S.-L. and Sekiguchi, T. (1965). Instantaneous Direct-Display System of Plasma Parameters by Means of Triple Probe. Journal of Applied Physics 36, 2363.

Chiu, G. S. (1995). Studies of magnetized plasmas interacting with neutral gas. $\mathrm{PhD}$ thesis, Princeton University.

Chung, K. S. (1990). A kinetic theory of ion collection by probe in flowing unmagnetized plasma. Journal of Applied Physics 69, 3451.

Coroniti, F. V. (1985). Explosive Tail Reconnection: The Growth and Expansion Phases of Magnetospheric Substorms. Journal of Geophysical Research 90, 7427 .

Davidson, R. C. and Gladd, N. T. (1975). Anomalous transport properties associated with the lower-hybrid-drift instability. Physics of Fluids 18, 1327.

Drake, J. F., Biskamp, D., and Zeiler, A. (1997). Breakup of the electron current layer during 3-D collisionless magnetic reconnection. Geophysical Research Letters 24, 2921.

Dungey, J. W. (1961). Interplanetary magnetic field and the auroral zones. Physical Review Letters 6, 47. 
EjIRI, A. and Miчамото, K. (1995). Ion-heating model during magnetic reconnection in reversed-field pinch plasmas. Plasma Physics and Controlled Fusion 37, 43.

Fiksel, G., Hartog, D. J. D., and Fontana, P. W. (1998). An optical probe for local measurements of fast plasma ion dynamics. Review of Scientific Instruments 69, 2024.

Frank, A. G. (1989). Formation, Evolution and Explosive Disruption of Current Sheets in Plasma. In Plasma Physics and Plasma Electronics, pages 131-169. Nova Science Publishers, Commack, New York. Edited by L. M. Kovrizhnykh.

FreidberG, J. P. (1987). Ideal Magnetohydrodynamics. Plenum Press, New York.

Fujisawa, A., Ji, H., Yamagishi, K., Shinohara, S., Toyama, H., and Miyamoto, K. (1991). Anomalous Ion Temperature and Plasma Resistance due to MHD Fluctuations in REPUTE-1 Reversed Field Pinch Plasmas. Nuclear Fusion 31, 1443.

Gekelman, W., Stenzel, R. L., and Wild, N. (1982). Magnetic field line reconnection experiments, 3. Ion acceleration, flows, and anomalous scattering. Journal of Geophysical Research 87, 101.

Giovanelli, R. G. (1939). The Relations Between Eruptions and Sunspots. Astrophysical Journal 89, 555.

Giovanelli, R. G. (1946). A theory of chromospheric flares. Nature 158, 81.

Golub, L. and Pasachoff, J. M. (1997). The Solar Corona. Cambridge University Press, Cambridge.

Griem, H. R. (1964). Plasma Spectroscopy. McGraw-Hill, New York.

HARRIS, E. G. (1962). On a Plasma Sheath Separating Regions of Oppositely Directed Magnetic Field. Il Nuovo Cimento 23, 115.

Howell, R. B. and Nagayama, Y. (1985). Ion energy measurements on a reversed-field pinch experiment using Doppler broadening. Physics of Fluids 28, 743.

Hsu, S. C., Fiksel, G., Carter, T. A., Ji, H., Kulsrud, R. M., and YamadA, M. (2000). Local Measurement of Non-Classical Ion Heating During Magnetic Reconnection, to be published in Physical Review Letters. 
Huba, J. D., Gladd, N. T., and Papadopoulos, K. (1978). Lower-HybridDrift Wave Turbulence in Distant Magnetotail. Journal of Geophysical Research 83, 5217 .

Hudis, M. and Lidsky, L. M. (1970). Directional Langmuir Probe. Journal of Applied Physics 41, 5011.

Hutchinson, I. H. (1987). Principles of Plasma Diagnostics. Cambridge University Press, New York.

Janev, R. K., Langer, W. D., Evans, K., and Post, D. E. (1987). Elementary Processes in Hydrogen-Helium Plasmas. Springer-Verlag, New York.

Ji, H., Toyama, H., Yamagishi, K., Shinohara, S., Fujisawa, A., and Miyamoto, K. (1991). Probe measurements in the REPUTE-1 reverse field pinch. Review of Scientific Instruments 62, 2326.

Ji, H., Yamada, M., Hsu, S., and Kulsrud, R. (1998). Experimental Test of the Sweet-Parker Model of Magnetic Reconnection. Physical Review Letters $\mathbf{8 0}, 3256$.

Ji, H., Yamada, M., Hsu, S., Kulsrud, R., Carter, T., and Zaharia, S. (1999). Magnetic reconnection with Sweet-Parker characteristics in twodimensional laboratory plasmas. Physics of Plasmas 6, 1743.

Kivelson, M. G. and Russell, C. T. (1995). Introduction to Space Physics. Cambridge University Press, Cambridge.

Kolb, A. C., Dobbie, C. B., and Griem, H. R. (1959). Field Mixing And Associated Neutron Production in a Plasma. Physical Review Letters 3, 5.

Kornack, T. W., Sollins, P. K., and Brown, M. R. (1998). Experimental observation of correlated magnetic reconnection and Alfvénic ion jets. Physical Review E 58, R36.

Kulsrud, R. (1997). The Plasma Resistivity in the MRX Reconnection Layer. Bulletin of the American Physical Society 42, 2028.

KulsRud, R. M. (1998). Magnetic reconnection in a magnetohydrodynamic plasma. Physics of Plasmas 5, 1599.

Kulsrud, R. M. and Carter, T. A. (2000). Private communication.

Laframboise, J. G. (1966). Theory of Spherical and Cylindrical Langmuir Probes in a Collisionless, Maxwellian Plasma at Rest. Technical Report UTIAS No. 100, Institute for Aerospace Studies, University of Toronto. 
Levinton, F. M. (1999). Private communication.

Little, E. M., Quinn, W. E., and Ribe, F. L. (1961). Effects of Ionization and Magnetic Initial Conditions on a Magnetically Compressed Plasma (Scylla). Physics of Fluids 4, 711.

Litvinenko, Y. E. (1996). Particle Acceleration in Reconnecting Current Sheets with a Nonzero Magnetic Field. Astrophysical Journal 462, 997.

Lovberg, R. H. (1965). Magnetic Probes. In Plasma Diagnostic Techniques, pages 69-112. Academic Press, New York. Edited by R. H. Huddlestone and S. L. Leonard.

Mandt, M. E., Denton, R. E., and Drake, J. F. (1994). Transition to whistler mediated magnetic reconnection. Geophysical Research Letters 21, 73.

Masuda, S., Kosugi, T., Hara, H., Tsuneta, S., and ogawara, Y. (1994). A loop-top hard X-ray source in a compact solar flare as evidence for magnetic reconnection. Nature $\mathbf{3 7 1}, 495$.

Mayo, R. M., Fernandez, J. C., Henins, I., Kirschenbaum, L. S., Munson, C. P., and Wysocki, F. J. (1991). Time of Flight Measurement of Ion Temperatures in Spheromaks. Nuclear Fusion 31, 2087.

McPherron, R. L. (1979). Magnetospheric substorms. Reviews of Geophysics and Space Physics 17, 657.

Narain, U. and Ulmschneider, P. (1996). Chromospheric and Coronal Heating Mechanisms 2. Space Science Reviews 75, 453.

Ohyabu, N., Okamura, S., and Kawashima, N. (1974). Strong ion heating in a magnetic neutral point discharge. Physics of Fluids 17, 2009.

Ono, Y., Inomoto, M., Okazaki, T., and Ueda, Y. (1997). Experimental investigation of three-component magnetic reconnection by use of merging spheromaks and tokamaks. Physics of Plasmas 4, 1953.

Ono, Y., Yamada, M., Akao, T., Tajima, T., and Matsumoto, R. (1996). Ion Acceleration and Direct Ion Heating in Three-Component Magnetic Reconnection. Physical Review Letters 76, 3328.

PARKer, E. N. (1957). Sweet's mechanism for merging magnetic fields in conducting fluids. Journal of Geophysical Research 62, 509. 
Peterson, B. J., Talmadge, J. N., Anderson, D. T., Anderson, F. S. B., and Shohet, J. L. (1994). Measurement of ion flows using an "unmagnetized" Mach probe in the interchangeable module stellerator. Review of Scientific Instruments 65, 2599.

PetscheK, H. E. (1963). Magnetic field annihilation. Technical Report SP-50, NASA.

Priest, E. R., Foley, C. R., Heyvaerts, J., Arber, T. D., Culhane, J. L., and Acton, L. W. (1998). Nature of the heating mechanism for the diffuse solar corona. Nature 393, 545.

Rogers, B., Drake, J., and Shay, M. (1999). 3D Collisionless Magnetic Reconnection. Bulletin of the American Physical Society 44, 65.

Scime, E., Cekic, M., Hartog, D. J., Hokin, S., Holly, D. J., and WATts, C. (1992a). Ion heating and magnetohydrodynamic dynamo fluctuations in the reversed-field pinch. Physics of Fluids B 4, 4062.

Scime, E., Hokin, S., Mattor, N., and Watts, C. (1992b). Ion Heating and Magnetohydrodynamic Dynamo Fluctuations in the Reversed-Field Pinch. Physical Review Letters 68, 2165.

Shay, M. A., Drake, J. F., Denton, R. E., and Biskamp, D. (1998). Structure of the dissipation region during collisionless magnetic reconnection. Journal of Geophysical Research 103, 9165.

Shay, M. A., Drake, J. F., Rogers, B. N., and Denton, R. E. (1999). The scaling of collisionless, magnetic reconnection for large systems. Geophysical Research Letters 26, 2163.

Somov, B. V. and Kosugi, T. (1997). Collisionless Reconnection and HighEnergy Particle Acceleration in Solar Flares. Astrophysical Journal 485, 859.

Speiser, T. W. (1965). Particle Trajectories in Model Current Sheets, 1. Analytical Solutions. Journal of Geophysical Research 70, 4219.

Speiser, T. W. (1970). Conductivity Without Collisions or Noise. Planetary and Space Sciences 18, 613.

SpItzer, L. (1962). Physics of Fully Ionized Gases. Interscience Publishers, New York.

Stangeby, P. C. (1984a). Measuring plasma drift velocities in tokamak edge plasmas using probes. Physics of Fluids 27, 2699. 
Stangeby, P. C. (1984b). Plasma sheath transmission factors for tokamak edge plasmas. Physics of Fluids 27, 682.

Stenzel, R. L. and Gekelman, W. (1981). Magnetic field line reconnection experiments, 1. Field topologies. Journal of Geophysical Research 86, 649.

Stenzel, R. L., Gekelman, W., and Wild, N. (1982). Magnetic field line reconnection experiments, 4. Resistivity, heating, and energy flow. Journal of Geophysical Research 87, 111.

Sweet, P. A. (1958). The neutral point theory of solar flares. In Electromagnetic Phenomena in Cosmical Physics, pages 123-134. Cambridge University Press, New York. Edited by B. Lehnert.

TAYLOR, J. B. (1974). Relaxation of toroidal plasma and generation of reverse magnetic fields. Physical Review Letters 33, 1139.

TAYlor, J. B. (1986). Relaxation and magnetic reconnection in plasmas. Reviews of Modern Physics 58, 741.

Trintchouk, F. (2000). Private communication.

Trintchouk, F., Skinner, C. H., and Levinton, F. (1999). Two-dimensional measurements of turbulent structures using laser-induced fluorescence. Bulletin of the American Physical Society 44, 249.

Tsuneta, S., Acton, L., Bruner, M., Lemen, J., Brown, W., Caravalho, R., Catura, R., Freeland, S., Jurcevich, B., Morrison, M., Ogawara, Y., Hirayama, T., and Owens, J. (1991). The Soft X-Ray Telescope for the Solar-A Mission. Solar Physics 136, 37.

Tuszewski, M. (1988). Field Reversed Configurations. Nuclear Fusion 28, 2033.

UzDensky, D. (1998). Theoretical Study of Magnetic Reconnection. PhD thesis, Princeton University.

VAsyliunas, V. M. (1975). Theoretical models of magnetic field line merging, 1. Reviews of Geophysics and Space Physics 13, 303.

von Goeler, S., Stodiek, W., and Sauthoff, N. (1974). Studies of Internal Disruptions and $\mathrm{m}=1$ Oscillations in Tokamak Discharges with Soft-X-Ray Techniques. Physical Review Letters 33, 1201. 
Watanabe, T.-H., Hayashi, T., and Sato, T. (1999). Modeling of magnetic island formation in magnetic reconnection experiment. Physics of Plasmas 6, 1253.

Weiss, P. (1999). The Sun Also Writhes. Science News 155, 200.

Wiese, W. L. (1965). Line Broadening. In Plasma Diagnostic Techniques, pages 265-317. Academic Press, New York. Edited by R. H. Huddlestone and S. L. Leonard.

Yamada, M., Chu, T. K., Jr, R. A. E., Janos, A. C., Levinton, F. M., Mayo, R. M., Motley, R. W., Nagata, M., Ono, Y., SAtomi, N., and UEDA, Y. (1990a). Experimental investigation of magnetic compression of a spheromak plasma. Physics of Fluids B 2, 3074.

Yamada, M., Furth, H. P., Hsu, W., Janos, A., Jardin, S., Okabayashi, M., Sinnis, J., Stix, T. H., and Yamazaki, K. (1981). Quasistatic Formation of the Spheromak Plasma Configuration. Physical Review Letters 46, 188.

Yamada, M., Ji, H., Hsu, S., Carter, T., Kulsrud, R., Bretz, N., Jobes, F., Ono, Y., and Perkins, F. (1997a). Study of driven magnetic reconnection in a laboratory plasma. Physics of Plasmas 4, 1936.

Yamada, M., Ji, H., Hsu, S., Carter, T., Kulsrud, R., Ono, Y., and Perkins, F. (1997b). Identification of Y-Shaped and O-Shaped Diffusion Regions During Magnetic Reconnection in a Laboratory Plasma. Physical Review Letters 78, 3117.

Yamada, M., Ji, H., Hsu, S., Carter, T., Kulsrud, R., and Trintchouk, F. (2000). Experimental investigation of the neutral sheet profile during magnetic reconnection, to be published in Physics of Plasmas.

Yamada, M., Ji, H., Jardin, S., Kulsrud, R., Mikkelsen, D., and Zweben, S. (1998). Proposed Program SPIRIT (Self-organized Plasma with Induction, Reconnection, and Injection Techniques). Bulletin of the American Physical Society 43, 1768.

Yamada, M., Ono, Y., Hayakawa, A., and Katsurai, M. (1990b). Magnetic Reconnection of Plasma Toroids with Cohelicity and Counterhelicity. Physical Review Letters 65, 721. 